University of Louisville

ThinkIR: The University of Louisville's Institutional Repository

Electronic Theses and Dissertations

$12-2008$

\title{
Modeling the relationship between air quality and intelligent transportation system (ITS) with artificial neural networks.
}

Dinesh Kumar Gupta 1965-

University of Louisville

Follow this and additional works at: https://ir.library.louisville.edu/etd

\section{Recommended Citation}

Gupta, Dinesh Kumar 1965-, "Modeling the relationship between air quality and intelligent transportation system (ITS) with artificial neural networks." (2008). Electronic Theses and Dissertations. Paper 549. https://doi.org/10.18297/etd/549

This Doctoral Dissertation is brought to you for free and open access by ThinkIR: The University of Louisville's Institutional Repository. It has been accepted for inclusion in Electronic Theses and Dissertations by an authorized administrator of ThinkIR: The University of Louisville's Institutional Repository. This title appears here courtesy of the author, who has retained all other copyrights. For more information, please contact thinkir@louisville.edu. 
MODELING THE RELATIONSHIP BETWEEN AIR QUALITY AND INTELLIGENT TRANSPORTATION SYSTEMS (ITS) WITH ARTIFICIAL NEURAL NETWORKS

By

Dinesh Kumar Gupta

B.S., Malaviya National Institute of Technology, Jaipur, India, 1986

M.S., Indian Institute of Technology, Kharagpur, India, 1988

A Dissertation

Submitted to the Faculty of the

Graduate School of the University of Louisville

In Partial Fulfillment of the Requirements

For the Degree of

Doctor of Philosophy

Department of Civil and Environmental Engineering

University of Louisville

Louisville, Kentucky

December 2008 


\section{MODELING THE RELATIONSHIP BETWEEN AIR QUALITY AND INTELLIGENT}

TRANSPORTATION SYSTEMS (ITS) WITH ARTIFICIAL NEURAL NETWORKS

By

Dinesh Kumar Gupta

B.S., Malaviya National Institute of Technology, Jaipur, India, 1986

M.S., Indian Institute of Technology, Kharagpur, India, 1988

A Dissertation Approved on

November 24, 2008

by the following Dissertation Committee:

Dissertation Director 


\section{DEDICATION}

This dissertation is dedicated to AWESOME-THREESOME sisters

Bunty,

Bitty,

$\&$

Sonu

who were so determined to get me a $\mathrm{PhD}$

in

USA.

Especially,

To

Bunty-Didi

who has morally supported me throughout this educational endeavor and provided me mental strength whenever I was weak. 


\section{ACKNOWLEDGEMENTS}

I profoundly express my gratitude to Dr. Louis F. Cohn, the chairman of my committee and dissertation supervisor, for his encouragement, valuable guidance, and constant support, mentally, morally and materially, during this research. He patiently guided me through the dissertation process, never accepting less than my best efforts.

Also I would like to convey my gratefulness to Dr. Mark N. French, a friend, philosopher and guide, for his continued support, approachability and intelligent suggestions throughout this study.

This dissertation would not have been possible without the help of Dr. Bhaskar, N. R., Dr. Harris R. A., and Dr. Desoky, A. H. who not only served as the members of my dissertation committee but also motivated and challenged me throughout my academic program. I thank them all from the bottom of my heart.

In addition, I would also like to thank EPA and USDOT for liberally using their web-sites for downloading the data, which were the lifeline of this study and were crucial for the completion of this research.

I can not forget the help and enthusiasm of entire faculty and staff of the Civil and Environmental Engineering Department. I thank them all, in all my sincerity, for their 
colleagueship, which made my days at UofL more pleasurable and stress-free than I could have imagined.

I would also like to thank my son, Prinesh, whose innocent and handsome face always reminded me of the need of acceleration in my work so that I could be with him once again as soon as possible.

Finally, I would like to thank my wife, Sanjeeta, for her continuous support and encouragement, especially when goings used to be tough. Without her hard work of supporting the family in India, I would not have been at peace of mind which was so essential for the completion of this endeavor. 


\begin{abstract}
MODELING THE RELATIONSHIP BETWEEN AIR QUALITY AND INTELLIGENT TRANSPORTATION SYSTEMS (ITS) WITH ARTIFICIAL NEURAL NETWORKS
\end{abstract}

\author{
Dinesh Kumar Gupta
}

November 24, 2008

Environmental or air quality impacts of Intelligent Transportation Systems (ITS) are very difficult to measure. Some researchers have attempted to quantify the effects of individual ITS application on emissions; yet, the effects of ITS as a whole on ambient air quality have not been investigated.

The objective of this research was to model the relationship between ITS and ambient air quality. The multiple Artificial Neural Networks (ANN) training with the data yielded a model for predicting the air quality. In addition, the ANN made the measurement of the effect of ITS on air quality possible.

Data pertaining to sixty US cities (urbanized area) were used for this research. Input variables used were related to transportation and local characteristics, and ITS applications. Output variables were the annual average concentrations of $\mathrm{CO}$, Ozone, and $\mathrm{NO}_{2}$ in ambient air. The K-fold cross validation technique was used to train the ANN. The results of ANN model were compared with that of a Multiple Regression (MR) model showing the supremacy of ANN over MR. The ANN model results show that the Mean Absolute Errors (MAEs) in prediction vary from 5 to $20 \%$. This variance is justified since the factors related with industries, which contribute significantly to air 
pollution, have not been taken into consideration in this study. There were some unusual findings: in contrast to the common assumptions, $\mathrm{NO}_{2}$ concentration increases with ITS intensity, and Ground Level Ozone concentration, in ambient air, seemed to be more transportation-dependent as compared with that of $\mathrm{CO}$ and $\mathrm{NO}_{2}$.

A recommendation for further research on this topic is to include more input variables, especially those which are related with industries, to improve the accuracy of prediction. Scientific experimentations have also been recommended to corroborate the unusual findings. 


\section{TABLE OF CONTENTS}

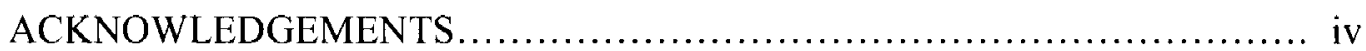

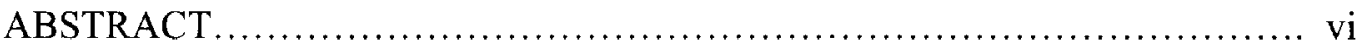

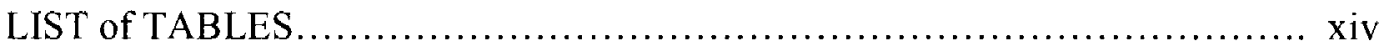

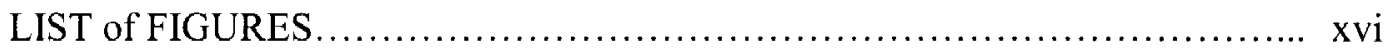

\section{CHAPTER}

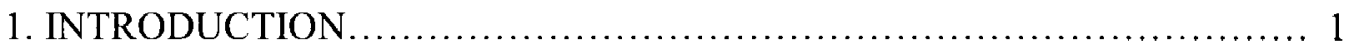

1.1 Background.................................................. 2

1.2 Research Objective........................................ 8

1.3 Potential Contribution of This Research....................... 9

2. LITERATURE REVIEW .......................................... 11

2.1 Previous Research............................................... 11

2.2 Ambient Air Pollution......................................... 21

2.2.1 Particle Pollution....................................... 22

2.2.2 Ground Level Ozone....................................... 23

2.2.3 Carbon monoxide......................................... 25

2.2.4 Nitrogen oxides........................................ 26

2.2.5 Sulfur dioxide........................................ 29

2.2 .6 Lead .................................................. 31

2.2.7 National Ambient Air Quality Standards (NAAQS)........ 32

2.2.8 How the Clean Air Act Reduces Air Pollution................. 34

2.3 Transportation and Air Quality................................ 35

2.3.1 Carbon monoxide..................................... 36

2.3.2 Hydrocarbons ........................................... 38

2.3.3 Nitrogen oxides...................................... 40

2.4 ITS Application Overview........................................ 41

2.4.1 Arterial Management................................ 42

viii 
2.4.1.1 Surveillance................................. 42

2.4.1.2 Traffic Control.............................. 42

2.4.1.3 Lane Management............................ 43

2.4.1.4 Parking Management........................... 43

2.4.1.5 Information Dissemination..................... 44

2.4.1.6 Enforcement................................... 44

2.4.2 Freeway Management Systems....................... 45

2.4.2.1 Surveillance................................. 46

2.4.2.2 Ramp Control............................... 46

2.4.2.3 Lane Management.............................. 46

2.4.2.4 Special Event Transportation Management........ 46

2.4.2.5 Information Dissemination..................... 47

2.4.2.6 Enforcement................................ 47

2.4.3 Transit Management Systems.......................... 47

2.4.3.1 Safety and Security......................... 47

2.4.3.2 Transportation Demand Management............. 48

2.4.3.3 Fleet Management............................... 48

2.4.3.4 Information dissemination...................... 49

2.4.4 Incident Management Systems........................ 50

2.4.4.1 Surveillance \& Detection....................... 50

2.4.4.2 Mobilization \& Response........................ 51

2.4.4.3 Information dissemination...................... 51

2.4.4.4 Clearance \& Recovery......................... 52

2.4.5 Emergency Management Systems........................ 52

2.4.5.1 Hazardous Materials Management.............. 52

2.4.5.2 Emergency Medical Services................... 53

2.4.5.3 Response \& Recovery........................ 54

2.4.6. Electronic Payment and Pricing........................ 54

2.4.6.1 Toll Collection............................. 55

2.4.6.2 Transit Fare Payment........................ 56

2.4.6.3 Parking Fee Payment....................... 56

2.4.6.4 Multi-use Payment............................... 56

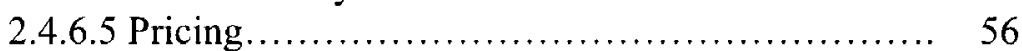

2.4.7 Traveler Information................................. 57

2.4.7.1 Pre-trip Information......................... 58

2.4.7.2 En-route Information.......................... 58

2.4.7.3 Tourism and Events.......................... 58

2.4.8 Information Management.............................. 58

2.4.8.1 Data Archiving............................... 59

2.4.9 Crash Prevention and Safety............................ 59

2.4.9.1 Road Geometry Warning Systems............... 61

2.4.9.2 Highway Rail Crossing Systems................. 61

2.4.9.3 Intersection Collision Warning................... 61

2.4.9.4 Pedestrian Safety............................... 61

2.4.9.5 Bicycle Warning Systems................... 62 
2.4.9.6 Animal Warning Systems....................... 62

2.4.10 Roadway Operations \& Maintenance.................. 62

2.4.10.1 Information Dissemination..................... 62

2.4.10.2 Asset Management............................ 63

2.4.10.3 Work Zone Management................... 64

2.4.11 Road Weather Management........................... 64

2.4.11.1 Surveillance, Monitoring, \& Prediction.......... 64

2.4.11.2 Information Dissemination................... 65

2.4.11.3 Traffic Control................................. 65

2.4.11.4 Response \& Treatment........................ 65

2.4.12 Commercial Vehicle Operations......................... 66

2.4.12.1 Credentials Administration.................... 67

2.4.12.2 Safety Assurance............................. 67

2.4.12.3 Electronic Screening.......................... 67

2.4.12.4 Carrier Operations \& Fleet Management......... 67

2.4.12.5 Security Operations........................... 68

2.4 .13 Intermodal Freight ................................ 69

2.4.13.1 Freight Tracking.............................. 69

2.4.13.2 Asset Tracking............................. 69

2.4.13.3 Freight Terminal Processes..................... 69

2.4.13.4 Drayage Operations....................... 70

2.4.13.5 Freight Highway Connector System............. 71

2.4.13.6 International Border Crossing Processes.......... 71

2.4.14 Collision Avoidance Systems............................ 71

2.4.14.1 Intersection Collision Warning................. 72

2.4.14.2 Obstacle Detection.............................. 72

2.4.14.3 Lane Change Assistance........................ 72

2.4.14.4 Lane Departure Warning...................... 73

2.4.14.5 Rollover Warning............................. 74

2.4.14.6 Road Departure Warning..................... 74

2.4.14.7 Forward Collision Warning..................... 74

2.4.14.8 Rear Impact Warning,............................ 74

2.4.15 Driver Assistance Systems............................ 75

2.4.15.1 Navigation/Route Guidance.................... 75

2.4.15.2 Driver Communication...................... 77

2.4.15.3 Vision Enhancement.......................... 77

2.4.15.4 Object Detection................................. 77

2.4.15.5 Adaptive Cruise Control........................... 77

2.4.15.6 Intelligent Speed Control....................... 78

2.4.15.7 Lane Keeping Assistance....................... 78

2.4.15.8 Roll Stability Control............................ 78

2.4.15.9 Drowsy Driver Warning ........................... 78

2.4.15.10 Precision Docking............................. 79

2.4.15.11 Coupling/Decoupling.......................... 79

2.4.15.12 On-board Monitoring....................... 79

2.4.16 Collision Notification Systems........................ 79 
2.4.16.1 Mayday/CAN .................................. 80

2.4.16.2 Advanced CAN ............................. 80

2.4.17 Other Applications ......................................... 81

2.4.17.1 Smart Glasses................................ 81

2.4.17.2 Real-time Rail Time Table on iPhone............. 81

2.4.17.3 Smart Roads.................................. 81

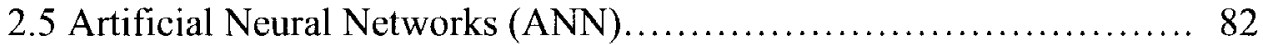

2.5.1 Biological Neuron and Artificial Neuron.................... 84

2.5.1.1 Biological Neuron.................................. 85

2.5.1.2 Artificial Neuron................................... 87

2.5 .2 ANN Features...................................... 88

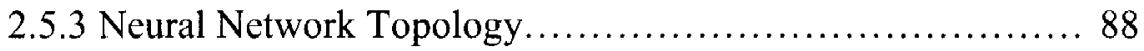

2.5.4 Activation Functions........................................ 89

2.5.5 Training an ANN ........................................ 91

2.5.5.1 Supervised Training.............................. 91

2.5.5.2 Unsupervised Training ............................ 91

2.5.6 Various issues with ANN .............................. 92

2.6 Kendall's Tau Test and Multiple-Regression (MR) .................. 92

2.6.1 Kendall's Tau Test................................. 92

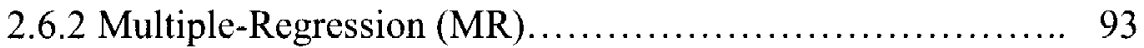

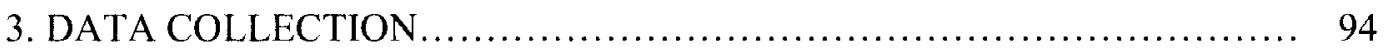

3.1 Air Data ............................................................... 95

3.2 Transportation/Highway Data................................... 96

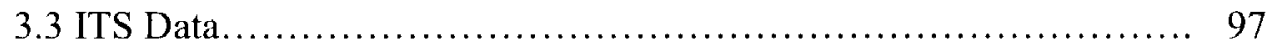

4. RESEARCH METHODOLOGY ........................................ 102

4.1 Data Normalization............................................... 102

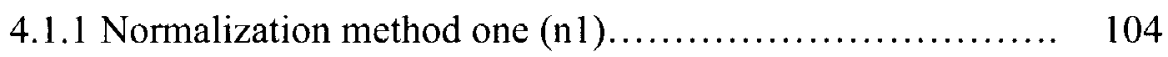

4.1.2 Normalization method two (n2) .................... 104

4.2 Reduction of ITS Application Variables......................... 105

4.2.1 Correlations........................................ 106

4.2.2 Conversion of seventeen ITS variables into one............ 108

4.3 Modeling....................................................... 110

4.3.1 ANN Modeling........................................ 111

4.3.1.1 Feedforward Neural Networks.................. 112

4.3.1.2 Network Architecture.......................... $\quad 113$

4.3.1.3 Training Algorithm (Learning Rule) ............ 114 
4.3.1.4 Transfer Function (Activation Function)........... 115

4.3.2 Multiple Linear regression Modeling................... 117

4.3.3 K-fold Cross-validation................................. 117

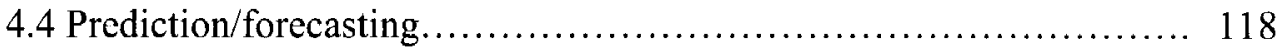

4.4.1 Removal of Outliers.................................. 118

4.4.2 ANN Modeling.......................................... 119

4.4.3 Multiple Regression (MR) Modeling.................... 120

4.5 Effect of ITS on Ambient Air Quality............................ 121

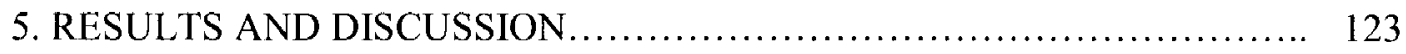

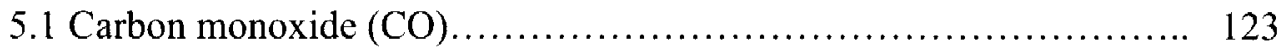

5.1.1 ANN Architecture selection............................ 124

5.1.1.1 Results....................................... 124

5.1.1.2 Discussion................................... 130

5.1.2 Comparison of Observed and Predicted values............. 132

5.1.2.1 Results.................................... 132

5.1.2.2 Discussion.................................. 141

5.1 .3 Effect of ITS on ambient air quality.................... 142

5.1 .3 .1 Results.................................. 142

5.1.3.2 Discussion.................................. 143

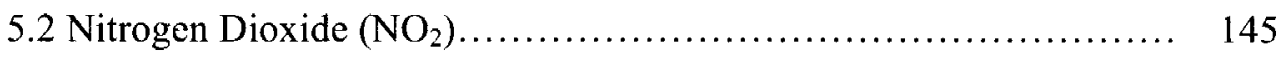

5.2.1 ANN Architecture selection............................ 145

5.2.1.1 Results........................................ 145

5.2.1.2 Discussion................................. 150

5.2.2 Comparison of Observed and Predicted values............. 152

5.2 .2 .1 Results......................................... 152

5.2.2.2 Discussion.................................... 161

5.2 .3 Effect of ITS on ambient air quality .................... 162

5.2.3.1 Results...................................... 162

5.2 .3 .2 Discussion............................... 163

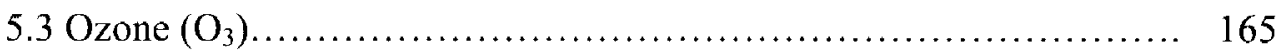

5.3.1 ANN Architecture selection............................. 165

5.3.1.1 Results...................................... 165

5.3.1.2 Discussion................................... 170

5.3.2 Comparison of Observed and Predicted values................ 172

5.3.2.1 Results....................................... 172

5.3.2.2 Discussion................................. 182

5.3.3 Effect of ITS on ambient air quality.................... 184

5.3.3.1 Results................................... 184 
6. CONCLUSIONS AND RECOMMENDATIONS ....................... 187

6.1 Conclusions.................................................... 187

6.2 Contributions to the Sum of Knowledge....................... 189

6.3 Recommendations for future research........................... 191

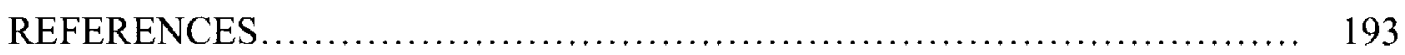

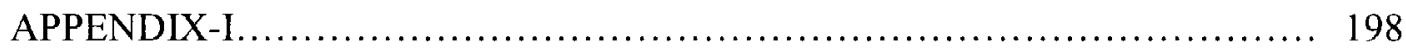

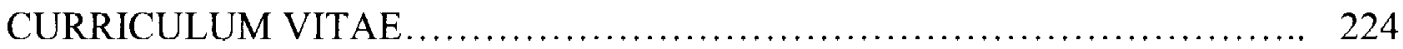




\section{LIST OF TABLES}

\section{TABLE}

1.1 Pollutants by Source......................................... 2

1.2 Emissions and Air Quality Trends (1990-1999).................. 4

2.1 National Ambient Air Quality Standards........................ 33

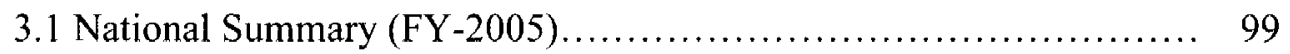

3.2 Variables....................................................... 100

4.1 Correlations.................................................. 107

4.2 Revised Variables......................................... 110

5.1 ANN model performance according to architecture and corresponding MAE measure (for data normalized by method-1) ................. 125

5.2 ANN model performance according to architecture and corresponding MAE measure (for modified data normalized by method-1)...........

5.3 ANN model performance according to architecture and corresponding MAE measure (for data normalized by method-2) ...................

5.4 ANN model performance according to architecture and corresponding MAE measure (for modified normalized data by method-2)...........

5.5 ANN model performance according to architecture and corresponding MAE measure (for data normalized by method-1).....................

5.6 ANN model performance according to architecture and corresponding MAE measure (for modified normalized data by method-1).

5.7 ANN model performance according to architecture and corresponding MAE measure (for data normalized by method-2). 
5.8 ANN model performance according to architecture and corresponding MAE measure (for modified normalized data by method-2)............

5.9 ANN model performance according to architecture and corresponding MAE measure (for data normalized by method-1) .................. 166

5.10 ANN model performance according to architecture and corresponding MAE measure (for modified data normalized by method -1 )........... 167

5.11 ANN model performance according to architecture and corresponding MAE measure (for data normalized by method-2).................. 168

5.12 ANN model performance according to architecture and corresponding MAE measure (for modified normalized data by method-2)............ 


\section{LIST OF FIGURES}

FIGURE

1.1 Comparison of Growth Areas and Emissions....................... 3

2.1 1999 National Emissions by Source: Carbon Monoxide............. 37

2.2 1999 National Emissions by Source: Carbon Monoxide

On-Road Mobile Sources................................... 37

2.31999 National Emissions by Source: Hydrocarbons................. 38

2.4 1999 National Emissions by Source: Hydrocarbons

On-Road Mobile Sources...................................... 39

2.51999 National Emissions by Source: Nitrogen Oxides............... 40

2.6 1999 National Emissions by Source: Nitrogen Oxides

On-Road Mobile Sources........................................ 41

2.7 Arterial Management Systems............................. 43

2.8 Freeway Management Systems................................. 45

2.9 Transit Management Systems................................. 49

2.10 Incident Management Systems.............................. 51

2.11 Emergency Management Systems.......................... 53

2.12 Electronic Payment and Pricing............................ 55

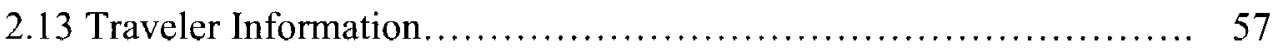

2.14 Information Management.................................. 59

2.15 Crash Prevention and Safety............................... 60 
2.16 Roadway Operations and Maintenance............................ 63

2.17 Roadway Weather Management............................. 66

2.18 Commercial Vehicle Operations.............................. 68

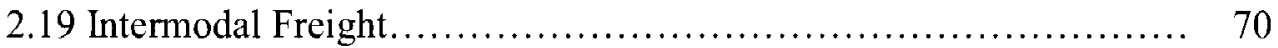

2.20 Collision Avoidance Systems................................ 73

2.21 Driver Assistance Systems..................................... 76

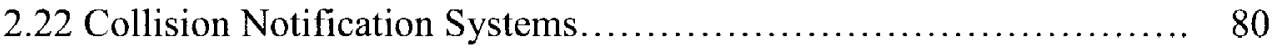

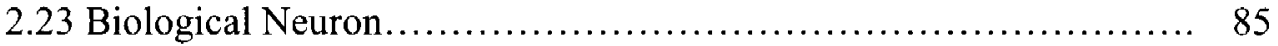

2.24 Artificial Neuron................................................ 87

2.25 Feed Forward Neural Networks................................ 89

2.26 Types of Activation Function............................... 90

5.1 ANN performance summary according to architecture and corresponding MAE (for data normalized by method-1) ............. 126

5.2 ANN performance summary according to architecture and corresponding MAE (for modified normalized data by method-1)......

5.3 ANN performance summary according to architecture and corresponding MAE (for normalized data by method-2). . .

5.4 ANN performance summary according to architecture and corresponding MAE (for modified normalized data by method-2).......

5.5 Observed versus Predicted values by ANN for normalized data by method-1

5.6 Observed and Predicted values by ANN for data normalized by method-1

5.7 Observed and Predicted values by ANN for data normalized by method-2.

5.8 Observed and Predicted values by ANN for data normalized by method- 2 
5.9 Observed and Predicted values by MR Model for data normalized by method- 1

5.10 Observed and Predicted values by MR Model for data normalized by method-1

5.11 Observed and Predicted values by MR Model for data normalized by method- 2 .

5.12 Observed and Predicted values by MR Model for data normalized by method- 2

5.13 Observed versus Predicted values by ANN model for modified normalized data by method- 1

5.14 Observed versus Predicted values by ANN model for modified normalized data by method- 1 .

5.15 Observed versus Predicted values by ANN model for modified normalized data by method- 2

5.16 Observed versus Predicted values by ANN model for modified normalized data by method- 2 .

5.17 Observed versus Predicted values by MR model for modified normalized data by method- 1

5.18 Observed versus Predicted values by MR model for modified normalized data by method-1...

5.19 Observed versus Predicted values by MR model for modified normalized data by method- 2 .

5.20 Observed versus Predicted values by MR model for modified normalized data by method- 2

$5.21 \%$ ITS (intensity of application) versus CO concentration in ambient air.

5.22 ANN performance summary according to architecture and corresponding MAE (for normalized data by method-1).

5.23 ANN performance summary according to architecture and corresponding MAE (for modified normalized data by method-1)...

5.24 ANN performance summary according to architecture and 
corresponding MAE (for normalized data by method-2)

5.25 ANN performance summary according to architecture and corresponding MAE (for modificd normalized data by method-2)... 149

5.26 Observed versus Predicted values by ANN for normalized data by method-1

5.27 Observed and Predicted values by ANN for data normalized by method-1.

5.28 Observed and Predicted values by ANN for data normalized by method- 2 .

5.29 Observed and Predicted values by ANN for data normalized by method- 2

5.30 Observed and Predicted values by MR Model for data normalized by method- 1

5.31 Observed and Predicted values by MR Model for data normalized by method-1

5.32 Observed and Predicted values by MR Model for data normalized by method- 2 .

5.33 Observed and Predicted values by MR Model for data normalized by method- 2

5.34 Observed versus Predicted values by ANN model for modified normalized data by method-1

5.35 Observed versus Predicted values by ANN model for modified normalized data by method- 1 .

5.36 Observed versus Predicted values by ANN model for modified normalized data by method- 2 .

5.37 Observed versus Predicted values by ANN model for modified normalized data by method- 2 ...

5.38 Observed versus Predicted values by MR model for modified normalized data by method-1

5.39 Observed versus Predicted values by MR model for modified normalized data by method- 1 
5.40 Observed versus Predicted values by MR model for modified normalized data by method $-2 \ldots \ldots \ldots \ldots \ldots \ldots \ldots \ldots \ldots \ldots \ldots . \ldots \ldots$

5.41 Observed versus Predicted values by MR model for modified

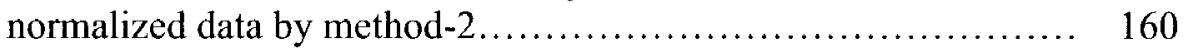

$5.42 \%$ ITS (intensity of application) versus $\mathrm{NO}_{2}$ concentration in ambient air.

5.43 ANN performance summary according to architecture and corresponding MAE (for normalized data by method-1).

5.44 ANN performance summary according to architecture and corresponding MAE (for modified normalized data by method-1)...

5.45 ANN performance summary according to architecture and corresponding MAE (for normalized data by method-2).

5.46 ANN performance summary according to architecture and corresponding MAE (for modified normalized data by method-2)... 169

5.47 Observed versus Predicted values by ANN for normalized data by method-1

5.48 Observed and Predicted values by ANN for data normalized by method-1

5.49 Observed and Predicted values by ANN for data normalized by method- 2 .

5.50 Observed and Predicted values by ANN for data normalized by method- 2 .

5.51 Observed and Predicted values by MR Model for data normalized by method- 1

5.52 Observed and Predicted values by MR Model for data normalized by method- 1

5.53 Observed and Predicted values by MR Model for data normalized by method- 2 .....

5.54 Observed and Predicted values by MR Model for data normalized by method- 2

5.55 Observed versus Predicted values by ANN model for modified normalized data by method- 1 
5.56 Observed versus Predicted values by ANN model for modified normalized data by method $-1 \ldots \ldots \ldots \ldots \ldots \ldots \ldots \ldots \ldots . . . \ldots \ldots$

5.57 Observed versus Predicted values by ANN model for

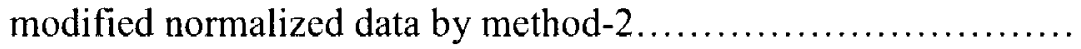

5.58 Observed versus Predicted values by ANN model for modified normalized data by method-2...

5.59 Observed versus Predicted values by MR model for modified normalized data by method-1

5.60 Observed versus Predicted values by MR model for modified normalized data by method-1

5.61 Observed versus Predicted values by MR model for modified normalized data by method-2...

5.62 Observed versus Predicted values by MR model for modified normalized data by method- 2 .

$5.63 \%$ ITS (intensity of application) versus $\mathrm{O}_{3}$ concentration in ambient air. 


\section{CHAPTER 1}

\section{INTRODUCTION}

Air pollution is a condition of the atmosphere, arising wholly or partly from the presence therein of one or more contaminants, that endangers the health, safety, or welfare of persons, interferes with normal enjoyment of life or property, endangers the health of animal life, or causes damage to plant life or property (Environment Canada, 1996). Air pollution is caused from two major sources: (1) stationary sources such as factories, industrial units, power plants, manufacturing facilities, and municipal waste incinerators, and (2) mobile sources or motor vehicles such as cars, trucks, and buses. Motor vehicles have been a major source of air pollution. Due to the ever-growing transportation activities, which are linked to the economic progress of a region, the road infrastructure of US has come under a pressure, resulting in congestion and delays and causing serious reduction in the mobility of the nation. The challenge now lies in improving the mobility of the nation without adversely affecting the air quality. Hence, it is important to explore transportation options that may result in potential mobility increase along with air quality benefits. Intelligent Transportation Systems (ITS) is believed to constitute one such class of strategies that could have significant air quality benefits. Quantification of these benefits and subsequently identifying the relationship between ITS and ambient air quality should, obviously, be an important part of any ITS assessment effort and decision-making in the perspective of ITS deployment. 


\subsection{Background}

The major pollutants which pose health hazards to human are Ground Level Ozone and Carbon Monoxide. Ground Level Ozone is the resultant of the complex reactions of NOx and VOC in the presence of light. Transportation is the biggest contributor to air pollution, as can be scen from Table 1.1.

Table 1.1 Pollutants by Source

\begin{tabular}{|c|c|c|c|c|}
\hline \multirow{2}{*}{ Pollutants } & \multicolumn{4}{|c|}{ Sources of Emissions } \\
\cline { 2 - 5 } & Transportation & $\begin{array}{l}\text { Fuel } \\
\text { Combustion }\end{array}$ & $\begin{array}{l}\text { Industrial } \\
\text { Processes }\end{array}$ & Miscellaneous \\
\hline $\mathrm{CO}$ & $78.6 \%$ & $6.0 \%$ & $5.4 \%$ & $10.0 \%$ \\
\hline $\mathrm{Lead}$ & $13.1 \%$ & $12.7 \%$ & $74.2 \%$ & - \\
\hline $\mathrm{NO}_{x}$ & $53.3 \%$ & $41.7 \%$ & $3.7 \%$ & $1.3 \%$ \\
\hline $\mathrm{VOC}$ & $43.5 \%$ & $5.0 \%$ & $47.2 \%$ & $4.4 \%$ \\
\hline $\mathrm{PM}_{10}$ & $25.4 \%$ & $38.6 \%$ & $36.0 \%$ & - \\
\hline $\mathrm{SO}_{2}$ & $7.1 \%$ & $85.1 \%$ & $7.7 \%$ & $0.1 \%$ \\
\hline
\end{tabular}

Source: EPA Emissions Trends Report (EPA 454/F-00-002)

To address air quality concerns, Congress first enacted the Air Pollution Control Act of 1955. This Act provided funds for federal research in air pollution. The Clean Air Act of 1963 was the first federal legislation regarding air pollution control. It established a federal program within the U.S. Public Health Service and authorized research into techniques for monitoring and controlling air pollution. In 1967, the Air Quality Act was enacted in order to expand federal government activities. In accordance with this law, enforcement proceedings were initiated in areas subject to interstate air pollution transport. As part of these proceedings, the federal government for the first time conducted extensive ambient monitoring studies and stationary source inspections. The 
Air Quality Act of 1967 also authorized expanded studies of air pollutant emission inventories, ambient monitoring techniques, and control techniques (EPA, 2008).

The enactment of the Clean Air Act of 1970 (1970 CAA) resulted in a major shift in the federal government's role in air pollution control. The act mandates state and local governments to develop strategies to address the problem, and set minimum air quality standards called National Ambient Air Quality Standards (NAAQS). The Clean Air Act was amended in 1977 to emphasize the need for coordination of air quality planning with the transportation planning process of metropolitan planning organizations (CAS Handbook, FDOT, 1996). This has helped in containing air pollution, as expected, in last three decades as can be seen from Figure 1.1. During 1970 to 2002, gross domestic product increased $164 \%$, while vehicle miles traveled increased $155 \%$, showing a close relationship of transportation activities with economic growth. Energy consumption and US population increased $42 \%$ and $32 \%$ respectively, whereas the total emissions of six principal air pollutants decreased $48 \%$.

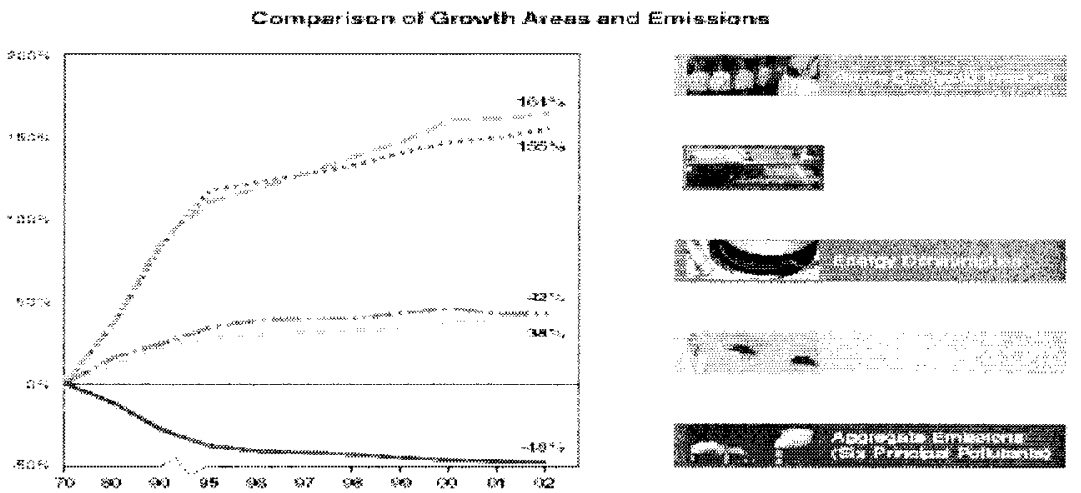

Source: Latest Findings on National Air Quality, 2002, EPA

Figure 1.1

Comparison of Growth Areas and Emissions 
The transportation sector, being a major source of air pollution, can play a very vital role in improving air quality. The Clean Air Act Amendments of 1990 gives a framework for developing air quality improvement plans. It has also put an additional requirement that transportation plans, programs, and projects must conform to the purpose of State Implementation Plans (SIPs) for the attainment of NAAQS. This additional requirement has resulted in a greater role of transportation officials in the development of air quality plans. It has also resulted in increased interface, both collaborative and conflicting, between the transportation and environmental communities (Shrouds, 1994). These developments over the past one and half decades have given some positive results as shown in Table 1.2.

Table 1.2 Emissions and Air Quality Trends (1990-1999)

\begin{tabular}{|c|c|c|}
\hline Pollutant & $\begin{array}{c}\text { Change in Air } \\
\text { Quality }\end{array}$ & $\begin{array}{c}\text { Change in } \\
\text { Emissions }\end{array}$ \\
\hline Lead & $-36 \%$ & $-7 \%$ \\
Nitrogen Dioxide & $-60 \%$ & $-23 \%$ \\
Ozone & $-10 \%$ & $+2 \%$ \\
Particulate Matter $\left(\mathrm{PM}_{10}\right)$ & $-4 \%(1 \mathrm{hr})$ & $-15 \%(\mathrm{VOC})$ \\
Sulphur Dioxide & $0 \%(8 \mathrm{hr})$ & $-16 \%$ \\
\end{tabular}

Source: EPA Emissions Trends Report (2) 
The challenge now rests in enhancing the quality of the air without unfavorably disturbing the mobility of the nation. The demand for travel is expected to increase at about $30 \%$ in the next few years. Therefore, to simply maintain congestion at the current levels and without the introduction of productivity-enhancing technologies, the capacity of the transportation system would have to be increased by $30 \%$. This would mean an addition of approximately 4,427 new miles (7,125 kilometers) of roadway every year, an unlikely event under current political and economic conditions (McGurrin, 1997).

In the above context, it is important to search transportation options that may result in significant mobility enhancement without adversely affecting air quality. Intelligent Transportation Systems (ITS) are one such class of options that are believed to have noteworthy air quality benefits. ITS applications could lead to capacity improvements with the same physical infrastructure by enhancing the efficiency of the transportation system. A 20-year life-cycle cost analysis for fifty major urban areas for the two options (capacity increase vis-a-vis ITS) indicated that the ITS-based investment would "reduce the need for new roads while saving approximately $35 \%$ of the required investment in urban highways" (ITS Benefit, FHWA, 1997).

Evaluation of ITS impacts on air quality is very difficult and further compounded by the fact that deployment of most ITS strategies has been relatively recent, and is largely still underway; therefore, the long-term relationships between these strategies and the parameters that affect air quality are not very clear.

Estimates of emissions changes are usually simulation based, using multiple scenarios with and without ITS services and under different traffic, weather and accident conditions to predict emissions changes. Before and after field data are sometimes used in 
combination with simulation; for example, GPS-equipped floating cars can be used to gather more detailed speed, stop, and acceleration data as inputs to estimating emissions. While much progress is being made in developing models integrating demand modeling, traffic network simulation and emissions modeling, there remain many challenges. A review of the state-of-practice of air quality evaluation of ITS outlined some of the following issues: (1) "emissions factors...(fail) to adequately capture the effects of vehicle-operating modes on mobile source emissions," (2) "current travel demand models and traffic flow simulation models are not sufficiently detailed for purposes of ITS evaluation," and (3) these models "also fail to provide the kind of inputs needed for use in modal emissions" (Mehta et al, 2001).

There have been attempts to quantify the effects of individual ITS applications on emission but to date, no research has been oriented towards determining the effect of ITS as a whole on the ambient air quality. The approaches to ITS evaluation that have been adopted in practice and reported in the ITS research literature exhibit a lot variation. One of the main concerns with ITS strategies is 'induced demand'. The concern is that the improved traffic operations and reduced travel times may lower the road users' perceived costs of vehicle travel and consequently induce additional vehicle traffic. This argument is based on the theory of induced growth in vehicle travel, which hypothesizes that an increase in the capacity of a specific corridor or road network will attract increased level of traffic (Dodder, 2005).

Also the emissions of CO, NOx and VOC are speed-dependent. The average speed, therefore, may prove to be an important factor while modeling the effects of ITS on air quality. How emissions are increasing and what is the rate of increase with average 
speed have not been clearly studied, and this is an obstruction in determining the effect of ITS on ambient air quality. Air-quality models, like transportation models, are far from perfect in replicating reality. In the real world, emissions from post-1980s cars are quite sensitive to the pattern and extent of vehicle accelerations. For example, under high acceleration conditions, emissions from new cars can approach the emission rates of precontrolled, 1960s-vintage vehicles. But, just as transportation models leave out the "induced demand" effect of transportation improvements, the air-quality models assume one pattern of driving, omitting from the analysis the effect of ITS improvements that reduce accelerations and decelerations (Burbank, 1995).

This is an important gap in the model that may penalize ITS strategies that smooth traffic flow. EPA's models assume one particular driving cycle: pattern of acceleration, speeds, and deceleration, under both the build and no-build scenarios. To the extent that an ITS strategy smoothes the area's driving pattern, reducing the extent of accelerations, it reduces/increases NOx and other emissions. But this will not be eviden: in the build/no-build comparison because EPA's models don't provide for it. So, even though the model results are legally binding for conformity purposes, they should be treated with caution until EPA undertakes more research and refines its models (Burbank, 1995).

In US and other countries, there is growing interest in and deployment of Intelligent Transportation Systems (ITS), from small-scale and relatively isolated applications, to entire ITS Architectures. At the same time, many of the cities, where the pressure to deploy ITS is felt strongest, also confront major air quality problems. ITS are 
believed to be the best options to address congestion in the face of physical, financial, and social limits on the ability to "build one's way out" of congestion with more highways(Dodder, 2005).

\subsection{Research Objectives}

In the context of the issues discussed in the Background section, this research addresses the following questions:

- In what ways can ITS have an impact, whether positive or negative, on overall air quality?

- Can air quality be predicted with a specific set of ITS applications and local characteristics?

- Are Intelligent Transport Systems (ITS) really helping in improving air quality or vice versa?

Only those priority air pollutants, which are closely linked with transportation activities, have been selected for analysis in this research. These are carbon monoxide $(\mathrm{CO})$, nitrogen dioxide $\left(\mathrm{NO}_{2}\right)$ and ozone $\left(\mathrm{O}_{3}\right)$.

This research has three main objectives that are detailed in the following section.

Objective 1: Develop a method to convert seventeen ITS applications related variables into a single variable.

Objective 2: Employ Artificial Neural Networks (ANN) to model the relationship between ITS plus the local characteristics (mostly related with roads) and average ambient concentration of each priority pollutant for prediction purposes. 
Objective 3: Determine the effect of ITS on the average ambient air concentration of each priority pollutant.

\subsection{Potential Contribution of this research}

Intelligent Transportation Systems (ITS) - the application of communications and information technology to surface transportation systems - has the potential to improve transportation in several ways, from safety to emissions reductions to travel time and reliability. ITS have become a worldwide technology, and many US cities are currently deploying ITS, from individual technologies to entire ITS Architectures. Whilə improving mobility is at the core of any ITS deployment, air quality concerns are such that ignoring possible air quality impacts of ITS technologies represents either a failure to leverage ITS for air quality improvements, or even a risk of running counter to air quality management efforts [Dodder, 2005]. While there is a growing number of studies evaluating the air quality benefits of ITS, there are important restrictions on the degree to which the results of these studies can be used to support planning of ITS in US cities and other nations. First, the challenges involved in modeling ITS air quality benefits mean that they normally focus on only one or two ITS technologies at a time. Second, air quality and mobility conditions diverge greatly across cities, meaning that air quality outcomes will also contrast widely. Third, present emission models are not able to take into account the effect of ITS on emission, especially the "induced demand" and the "effect on emissions with the change of average speed due to ITS". Finally, since the general public is more concerned with the ambient air quality (AAQ) rather than emissions, no models are available which could directly relate ITS to AAQ. 
To date, there have been no efforts to address these issues in totality. This research provides a new approach to overcome these issues. It specifies an innovative and easy methodology to assess the effect of ITS on air quality. In the future, when more data of ITS will be available, it should produce a modeling tool - combined with more variables (e.g., contribution of industry to air pollution) - to improve accuracy in quantifying the effects of ITS on ambient air quality. 


\section{CHAPTER II}

\section{LITERATURE REVIEW}

\subsection{Previous Research}

The Intelligent Transportation Systems Joint Program Office (ITS JPO) of the U.S. Department of Transportation (U.S. DOT) has developed an ITS Benefits Database and has categorized benefits into six goal areas: safety, mobility, productivity, efficiency, energy \& environment, and customer satisfaction. The air quality and energy impacts of ITS services are very important considerations, particularly for non-attainment areas. In most cases, environmental benefits can only be estimated by the use of analysis and simulation. The problems related to regional measurement include the small impact of individual projects and large numbers of exogenous variables including weather, contributions from non-mobile sources, air pollution drifting into an area from other regions, as well as the time-evolving nature of ozone pollution. Small-scale studies generally show positive impacts on the environment. These impacts result from smoother and more efficient flows in the transportation system. However, environmental impacts of travelers reacting to large-scale deployment in the long term are not well understood. Therefore, designing a suitable frame work for evaluating the air quality benefits is a challenging task (Mehta et al., 2001). 
There have been some methodological developments that have taken place in past in air quality evaluation of individual ITS applications. One of the earliest studies found to emphasize the effect of ITS on emission was a model, which indicated that changes in travel behavior due to better traveler information in Boston, Massachusetts would result in a 25 percent reduction in volatile organic compounds, a 1.5 percent decline in nitrous oxide, and a 33 percent decrease in carbon monoxide. While these estimates were significant for participating drivers, they represented only 28,800 daily trips in the Boston metropolitan area which had 2.9 million registered drivers (Tech Environmental, Inc., 1993).

The Automated Traffic Surveillance and Control Program (ATSAC) in Los Angeles, California, operated computerized signal control systems since 1984. As of 1994, it included 1,170 intersections, and 4509 detectors for signal timing optimization. It reportedly decreased fuel consumption 13 percent, and decreased air emissions 14 percent (Los Angeles DOT, 1994).

Beginning in September of 1990 , the SCOOT system implemented in Toronto, Canada covered 3 signal networks encompassing 75 signalized intersections. An on-street evaluation conducted from May-June 1993 found a 5.7 percent average decrease in fuel consumption, a 3.7 percent average decrease in hydrocarbons, and a 5.0 percent average decrease in Carbon monoxide emissions (Traffic Technology, 1995).

As part of the Congestion Mitigation and Air Quality Improvement Program (CMAQ), the San Francisco freeway service patrol has been in operation since August 1992. As of January 1997, the program has assisted more than 90,000 drivers. It has decreased air pollution and reduced fuel consumption by helping to reduce the effects of 
incident caused congestion, start-and-stop travel and vehicle idling. Estimates indicate a reduction in $32 \mathrm{~kg} /$ day of hydrocarbons, $322 \mathrm{~kg} /$ day of CO emissions, and NOx is reduced by $798 \mathrm{~kg} /$ day (FHWA, US DOT, 1996).

The City of Richmond installed a 4.7 million dollar advanced signal system at 262 signalized intersections and evaluated its impact on the central business district (CBD). The system was designed to coordination signal timing on four routes having independent signal timing plans. Timing was optimized using a system event schedule and the TRANSYT-7F signal timing optimization program. The timing plans were implemented using a central computer system and subsequently fine tuned based on input from drivers who compared traffic flow, signal phasing, splits, and offsets to platoon progression diagrams for each route. Field data were collected during peak periods using a test vehicle equipped with an automatic data collection system to record travel times, delays, stops, cmissions, and fuel consumption before and after system deployment. A series of runs performed on representative test sections on each route indicated that the fuel consumption decreased 10 to 12 percent and emissions decreased 5 to 22 percent (Hetrick \&McCollough, 1996).

Bilspedition Transport \& Logistics (BTL) of Scandinavia used GPS-based tracking of vehicles in combination with remotely-accessed on-board computers to raise the productivity of its fleet operations in southern and central Sweden. It reportedly cut wasted mileage and emissions, and brought a 15 percent increase in freight carried (Bunting, 1997).

The evaluation of air quality benefits from implementing automatic vehicle identification (AVI) technology for electronic toll collection (ETC) produced some 
positive results. Located in Orlando, Florida, the Orlando-Orange County Expressway Authority (OOCEA) operates eleven mainline toll plazas. The busiest, the Holland East Toll Plaza, includes fourteen toll lanes. Nine of which are used for peak direction travel. A before-after study on the Express Pass (E-PASS) implementation of AVI based ETC at the toll plaza was conducted to evaluate the reduction in vehicle emissions. Specifically, the reductions in Carbon monoxide (CO), hydrocarbons ( $\mathrm{HC})$, and Nitrogen Oxides (NOx) were evaluated at the toll plaza.

Using the MOBILE5a emission model, and data collected, it was shown that even with the increased volumes at the Holland East Toll Plaza, vehicle emissions were reduced. An overall average reduction in Carbon Monoxide by 7.29 percent and $\mathrm{HC}$ by 7.19 percent resulted from the simulation. However, NOx increased by 33.77 percent (Klondzinski et al., 1998).

A transit signal priority system in Southampton, England reduced bus fuel consumption by 13 percent, lowered bus emissions by 13 to 25 percent, increased fuel consumption for other vehicles by 6 percent, and increased the emissions of other vehicles up to 9 percent (TR Lab, 1999).

A pilot Electronic Toll Collection (ETC) project was installed and tested by Caltrans on the Carquinez Bridge. This bridge was chosen as the location because capacity at the toll plaza was sufficient to handle peak demands with booths out of service during the ETC demonstration. The report estimated a person-time savings of 79,919 hours (per year) or about $\$ 1.07$ million. Emission reductions were estimated at 9.82 million grams of $\mathrm{CO}, 1.06$ million grams of $\mathrm{NOx}$, and 0.46 million grams of $\mathrm{HC}$ per year (Gillen et al., 1999). 
A simulation study indicated that integrating traveler information with traffic and incident management systems in Seattle, Washington could reduce emissions by 1 to 3 percent, lower fuel consumption by 0.8 percent, and improve fuel economy by 1.3 percent (Wunderlich et al., 1999).

In Michigan, a study examined the safety, performance, user-acceptance, and deployment of intelligent cruise control (ICC) systems. The findings were based on a field operational test (FOT) conducted by the National Highway Traffic Safety Administration (NHTSA) and the University of Michigan Transportation Research Institute (UMTRI). The FOT involved 108 volunteer test drivers and 10 ICC-equipped Chrysler Concordes. The testing was performed between July 1996 and September 1997 , and the results were analyzed by Volpe. The analysis indicated that the technology reduced fuel consumption and emissions by limiting throttle fluctuations (Koziol et al., 1999).

An adaptive signal control system in Toronto, Canada reduced vehicle emissions by three to six percent. Also it lowered fuel consumption by four to seven percent (Greenough and Kelman, 1999).

In Torino, Italy, a simulation study found that an automated speed control system designed to optimize travel speeds between green lights reduced fuel consumption by 8.3 to 13.8 percent. It also reduced $\mathrm{CO}_{2}$ emissions by 3.9 to 5.4 percent, reduce hydrocarbon emissions by 4.2 to 6.9 percent, and reduce NOx emissions by 7.9 to 11.3 percent (Gianguido and Lorenzo Mussone, 1999). 
Optimizing signal timing at 700 intersections in the Tysons Corner area of Northern Virginia lead to a 9 percent reduction in fuel consumption. This also resulted in a 134,600 kilogram decrease in annual emissions (White et al., 2000).

In Arizona, traffic signal coordination among two jurisdictions contributed to a 1.6 percent reduction in fuel consumption. But it also led to a 1.2 increase in carbon monoxide emissions (Zimmerman, 2000).

Arterial traffic flow data were included in the traveler information system in Seattle, Washington. Simulation results indicated that vehicle emissions could be reduced by two percent (Jensen et al., 2000).

A study used field experiments and simulation models to quantify the environmental benefits of intelligent cruise control (ICC) vehicles. Field tests were conducted using one ICC vehicle and two other manually operated vehicles in a single lane of freeway traffic. During the field trials driver responses and vehicle dynamics were recorded as they followed a lead vehicle with a pre-programmed speed profile (aggressive-rapid-acceleration or smooth-acceleration). The ICC vehicle trailed the other vehicles at different positions and implemented a smoothing effect to decrease the variance between the acceleration and deceleration extremes exhibited by the manually operated vehicles. Information from each field test was then input into a simulation model to measure net changes in fuel consumption and emissions. Emissions measured included unburned hydrocarbons (HC), Carbon monoxide (CO), Carbon dioxide (CO2), and the oxides of Nitrogen (NOx). The smoothing of traffic flow by the ICC vehicle significantly reduced emissions and the fuel consumption of manual traffic (Bose and Ioannou, 2001). 
A research project to document the benefits of anti-icing techniques and road weather information system (RWIS) technologies was initiated under the National Cooperative Highway Research Program (NCHRP). Winter maintenance personnel indicated that road weather information systems decrease salt usage, and anti-icing techniques limit damage to roadside vegetation, groundwater, and air quality (Boselly, 2001).

A study examined the impacts of ARTIMIS (Advanced Regional Traffic Interactive Management and Information System) in metropolitan areas of Northern Kentucky and Cincinnati, Ohio. As of 1999, ARTIMIS consisted of closed circuit TV cameras (CCTV), portable dynamic message signs (DMS), highway advisory radio (HAR), freeway and ramp reference markers, freeway service patrols, time-saving incident investigation equipment, and advanced traveler advisory telephone services. Modeling found emissions reductions of 3.7 to 4.6 percent due to an advanced transportation management and traveler information system serving northern Kentucky and Cincinnati (Jeannotte, 2001).

This study was conducted in September of 2000 to evaluate the New Jersey Turnpike Authority (NJTA) E-ZPass electronic toll collection system. The study consisted of an extensive data collection effort that involved measuring traffic counts, queue lengths, lane configurations, and transaction times during peak periods at 27 toll locations. Field observations were collected at each toll station during peak periods and the results were evaluated against NJTA toll collection records. The peak period data was entered into a TOLLSIM micro-simulation model in order to generate 24 -hour profiles representative of total queue length and average vehicle-class delay before and after E- 
ZPass deployment at each station. Emission factors such as vehicle type, average vehicle approach speed, acceleration and deceleration rates, and length of time in queue were used to evaluate system-wide air-quality impacts. The E-ZPass electronic toll collection system on the New Jersey Turnpike reduced delay for all vehicles by 85 percent saving an estimated 1.2 million gallons of fuel each year and eliminating approximately 0.35 tons of VOC and 0.056 tons NOx per weekday (Wilbur Smith Associates, 2001).

A study examined changes in vehicle emissions as a result of electronic toll collection at three major toll stations (Fort McHenry Tunnel, Baltimore Harbor Tunnel, and Francis Scott Key Bridge) outside Baltimore, Maryland. The system used toll tag readers and in-vehicle transponders to automatically execute toll transactions as vehicles passed through toll booths. Computer models were used to quantify emissions of hydrocarbons $(\mathrm{HC})$, Carbon monoxide (CO), and Nitrogen oxides (NOx), before and after the system was deployed. An evaluation indicated these systems reduced environmentally harmful emissions by 16 to 63 percent (Saka and Agboh, 2002).

A study used the INTEGRATION simulation model to estimate the impact of implementing transit signal priority (TSP) on a 3.95 mile section Columbia Pike in Arlington, Virginia. Baseline corridor and cross street traffic flows were determined from field data collected between June 12 and 14, 2000. Saturated traffic flow parameters were estimated based on corridor geometry. During the A.M. peak period, transit signal priority on an arterial route in Arlington, Virginia could increase carbon monoxide emissions by 5.6 percent and decrease nitrogen emissions by 1.7 percent (Dion et al., 2002). 
Simulation of a transit signal priority system in Helsinki, Finland indicated that fuel consumption decreased by 3.6 percent. Also Nitrogen oxides were reduced by 4.9 percent, Carbon monoxide decreased by 1.8 percent, hydrocarbons declined by 1.2 percent, and particulate matter decreased by 1.0 percent (Lehtonen and Kulmala, 2002). In response to Onondaga County not meeting air quality standards in 1993, the City of Syracuse implemented the Signal Interconnect Design Project. The project standardized 145 intersections within the City of Syracuse and optimized the signal timing in an attempt to reduce automobile emissions by creating a more efficient network. By implementing coordinated signal timing on the arterial network in Syracuse, New York; total fuel consumption was reduced by 9 to 13 percent, average fuel consumption declined by 7 to 14 percent, average vehicle emissions decreased by 9 to 13 percent (Harris, 2003).

Researchers at Oak Ridge National Laboratory estimate that poor signal timing causes 296 million vehicle hours of delay. Appropriate timing of traffic signals can decrease congestion, improve air quality, reduce fuel consumption, and minimize aggressive driving behavior. Optimizing signal timing produces average benefit-to-cost ratios that approach 40 to 1 . Across the nation, traffic signal retiming programs have resulted in travel time and delay reductions of 5 to 20 percent and fuel savings of 10 to 15 percent. In Oakland County, Michigan retiming 640 traffic signals during a two-phase project resulted in Carbon monoxide reductions of 1.7 and 2.5 percent, Nitrogen oxide reductions of 1.9 and 3.5 percent, and hydrocarbon reductions of 2.7 and 4.2 percent (Halkias and Schauer, 2004). 
The Georgia DOT Intelligent Transportation System, known as NaviGAtor, covers 140 freeway miles in the Atlanta metropolitan area. The NaviGAtor system includes a traffic management center (TMC), freeway management components, advanced traveler information systems, and an incident management program. TMC operators use vehicle detectors, closed circuit television (CCTV) cameras, dynamic message signs (DMS), and ramp meters to collect traffic data and manage incidents. When TMC operators identify an incident, they dispatch a Highway Emergency Response Operator (HERO) to provide motorist assistance or traffic control, and disseminate traveler information via DMS, the NaviGAtor web site, and a telephone information service. This incident management program reduced annual fuel consumption by 6.83 million gallons, and contributed to decreased emissions: 2,457 tons less Carbon monoxide, 186 tons less hydrocarbons, and 262 tons less Nitrous oxides (Guin, et al., 2006).

Congestion charging in London improves efficiency, reduces pollution, and raises revenue for transit improvements. The program requires motorists to pay a fee of $£ 8$ per day to drive within the inner city of London on workdays between 7:00 AM and 6:30 PM. Motorists can buy a prepaid weekly, monthly, or annual pass and save 15 percent, or buy a daily pass and pay full price. Residents receive a 90 percent discount; however, motorcycles, licensed taxis, vehicles used by disabled people, some alternative fuel vehicles, buses, and emergency vehicles are exempt. Congestion charging in London resulted in pollutant emission reductions: 8 percent for oxides of nitrogen, 7 percent for airborne particulate matter, and 16 percent for carbon dioxide (Transport for London, 2007). 


\subsection{Ambient Air Pollution}

Six common air pollutants (also known as "criteria pollutants") are found all over the United States. They are particle pollution (often referred to as particulate matter), ground-level ozone, carbon monoxide, sulfur oxides, nitrogen oxides, and lead. These pollutants can harm your health and the environment, and cause property damage. Of the six pollutants, particle pollution and ground-level ozone are the most widespread health hazards. Details about these pollutants are discussed below.

EPA calls these pollutants "criteria" air pollutants because it regulates them by developing human healthbased and/or environmentally-based criteria (science based guidelines) for setting permissible levels. The set of limits based on human health is called primary standards. Another set of limits intended to prevent environmental and property damage is called secondary standards. A geographic area with air quality that is cleaner than the primary standard is called an "attainment" area; areas that do not meet the primary standard are called "nonattainment" areas.

EPA has been developing programs to cut emissions of these commonly found air pollutants since the Clean Air Act was passed in 1970. It's a big job, and although a great deal of progress has been made, it will take time to make the air healthy throughout the country. There are still several areas of the country, including many large cities, that are classified as nonattainment for at least one of the six common pollutants. Despite continued improvements in air quality, millions of people live in areas with unhealthy levels of pollution. 


\subsubsection{Particle Pollution}

Particle pollution, also known as particulate matter (PM), includes the very fine dust, soot, smoke, and droplets that are formed from chemical reactions, and produced when fuels such as coal, wood, or oil are burned. For example, sulfur dioxide and nitrogen oxide gases from motor vehicles, electric power generation, and industrial facilities react with sunlight and water vapor to form particles. Particles may also come from fireplaces, wood stoves, unpaved roads, crushing and grinding operations, and may be blown into the air by the wind.

EPA scientists and other health experts are concerned about particle pollution because very small or "fine" particles can get deep into the lungs. These fine particles, by themselves, or in combination with other air pollutants, can cause increased emergency room visits and hospital admissions for respiratory illnesses, and tens of thousands of deaths each year. They can aggravate asthma, cause acute respiratory symptoms such as coughing, reduce lung function resulting in shortness of breath, and cause chronic bronchitis.

The elderly, children, and asthmatics are particularly vulnerable to health problems caused by breathing fine particles. Individuals with pre-existing heart or lung disease are also at an increased risk of health problems due to particle pollution. Particles also cause fog reducing visibility in places like national parks and wilderness areas that are known for their picturesque vistas. These are places where one expects to see clearly for long distances. In many parts of the US, pollution has reduced the distance and clarity of vision by 70 percent (Environmental Protection Agency (EPA), 2008). 
Fine particles can remain suspended in the air and travel long distances with the wind. For example, over 20 percent of the particles that form haze in the Rocky Mountains National Park have been estimated to come from hundreds of miles away (EPA, 2008).

Particles also make buildings, statues and other outdoor structures dirty. Trinity Church in downtown New York City was black until a few years ago, when cleaning off almost 200 years worth of soot brought the church's stone walls back to their original light pink color (EPA, 2008).

Before the 1990 Clean Air Act went into effect, EPA set limits on airborne particles smaller than 10 micrometers in diameter called PM10. These are tiny particles (seven of these particles lined up next to each other would cover a distance no wider than a human hair). Research has indicated that even smaller particles (1/4 the size of a PM10 particle) are more likely to harm human health. So in 1997, EPA published limits for fine particles, called PM2.5. To reduce particle levels, additional controls are being required on a variety of sources including power plants and diesel trucks.

\subsubsection{Ground-level Ozone}

Ground-level ozone is a primary component of smog. Ground-level ozone can cause human health problems and harm forests and agricultural crops. Repeated contact with ozone can make people more vulnerable to respiratory infections and lung inflammation. It also can aggravate pre-existing respiratory diseases, such as asthma. Children are at risk from ozone pollution because they are outside, playing and 
exercising, during the summer days when ozone levels are at their highest. They also can be more susceptible because their lungs are still developing. People with asthma and even active healthy adults, such as construction workers, can experience a reduction in lung function and an increase in respiratory symptoms (chest pain and coughing) when exposed to low levels of ozone during periods of moderate exertion (EPA, 2008).

The two types of chemicals that are the chief ingredients in forming ground-level ozone are called volatile organic compounds (VOCs) and nitrogen oxides (NOx). VOCs are released by cars burning gasoline, petroleum refineries, chemical manufacturing plants, and other industrial facilities. The solvents used in paints and other consumer and business products consist of VOCs. The 1990 Clean Air Act has resulted in changes in product formulas to reduce the VOC content of those products. Nitrogen oxides (NOx) are produced when cars and other sources like power plants and industrial boilers burn fuels such as gasoline, coal, or oil. The reddish-brown color, when it is smoggy, comes from the nitrogen oxides (EPA, 2008).

The pollutants that react to form ground-level ozone literally cook in the sky during the hot summertime season. It takes time for smog to form-several hours from the time pollutants get into the air until the ground-level ozone reaches unhealthy level.

Weather and the topogaraphy of the land (for example, hills around a valley, high mountains between a big industrial city and suburban or rural areas) help determine where ground-level ozone goes and how worse it gets. When temperature inversions occur (warm air stays trapped near the ground by a layer of cooler air) and winds are calm, high concentrations of groundlevel ozone may continue for several days at a time. 
As traffic and other sources add more ozone-forming pollutants to the air, the groundlevel ozone gets worse (EPA, 2008).

\subsubsection{Carbon monoxide}

$\mathrm{CO}$ is a colorless and odorless gas that is formed when carbon in fuel is not burned completely. It is a component of motor vehicle exhaust, which contributes about 56 percent of all $\mathrm{CO}$ cmissions nationwide. Other non-road engines and vehicles (such as construction equipment and boats) contribute about 22 percent of all $\mathrm{CO}$ emissions nationwide. Higher levels of $\mathrm{CO}$ generally occur in areas with heavy traffic congestion. In cities, 85 to 95 percent of all $\mathrm{CO}$ emissions may come from motor vehicle exhaust. Other sources of $\mathrm{CO}$ emissions include industrial processes (such as metals processing and chemical manufacturing), residential wood burning, and natural sources such as forest fires. Woodstoves, gas stoves, cigarette smoke, and unvented gas and kerosene space heaters are sources of $\mathrm{CO}$ indoors. The highest levels of $\mathrm{CO}$ in the outside air typically occur during the colder months of the year when inversion conditions are more frequent (EPA, 2008). The air pollution becomes trapped near the ground beneath a layer of warm air. Carbon monoxide can cause harmful health effects by reducing oxygen delivery to the body's organs (like the heart and brain) and tissues. The health threat from lower levels of $\mathrm{CO}$ is most serious for those who suffer from heart disease, like angina, clogged arteries, or congestive heart failure. For a person with heart disease, a single exposure to $\mathrm{CO}$ at low levels may cause chest pain and reduce that person's ability to exercise; repeated exposures may contribute to other cardiovascular effects. Even healthy people can be affected by high levels of $\mathrm{CO}$. People who breathe high levels of $\mathrm{CO}$ can develop vision problems, reduced ability to work or learn, reduced manual dexterity, and 
difficulty performing complex tasks. At extremely high levels, $\mathrm{CO}$ is toxic and can cause death. $\mathrm{CO}$ contributes to the formation of smog ground-level ozone, which can trigger serious respiratory problems (EPA, 2008).

Reducing emissions of $\mathrm{CO}$ is an important part of EPA's policy for cleaner air. The U.S. Environmental Protection Agency (EPA), states, and local governments work as partners to decrease emissions of $\mathrm{CO}$. EPA's main strategy to reduce $\mathrm{CO}$ has been to establish national ambient air quality standards, to require national controls for motor vehicle emissions, and to require reductions from large industrial facilities. Starting in the early 1970's, EPA has set national standards that have considerably reduced emissions of CO. Since 1970, CO emissions from on-road vehicles (which include cars, motorcycles, light- and heavy-duty trucks) have been reduced by over 40 percent. The greatest reductions have been in emissions from cars (nearly 60 percent).

\subsubsection{Nitrogen oxides}

NOx is the generic term for a group of highly reactive gases, all of which consist of nitrogen and oxygen in varying amounts. Many of the nitrogen oxides are colorless and odorless. However, one common pollutant, nitrogen dioxide $\left(\mathrm{NO}_{2}\right)$ along with particles in the air can often be seen as a reddish-brown layer over many urban areas. NOx causes a wide variety of health and environmental impacts because of various compounds and derivatives in the family of nitrogen oxides, including nitrogen dioxide, nitric acid, nitrous oxide, nitrates, and nitric oxide. Ground-level Ozone (Smog) - is formed when NOx and volatile organic compounds (VOCs) react in the presence of sunlight. Millions of Americans live in areas that do not meet the health standards for ozone. Other impacts 
from ozone include damage to vegetation and crop yields. NOx and sulfur dioxide react with other substances in the air to form acids which fall to earth as rain, fog, snow or dry particles. Some may be carried by wind for hundreds of miles. Acid rain damages; causes deterioration of cars, buildings and historical monuments; and causes lakes and streams to become acidic and unsuitable for many fish. NOx reacts with ammonia, moisture, and other compounds to form nitric acid and related particles. Human health concerns include effects on breathing and the respiratory system, damage to lung tissue, and premature death. Small particles penetrate deeply into sensitive parts of the lungs and can cause or worsen respiratory disease such as emphysema and bronchitis, and aggravate existing heart disease. Increased nitrogen loading in water bodies, particularly coastal estuaries, upsets the chemical balance of nutrients used by aquatic plants and animals. Additional nitrogen accelerates "eutrophication," which leads to oxygen depletion and reduces fish and shellfish populations. NOx emissions in the air are one of the largest sources of nitrogen pollution in the Chesapeake Bay. One member of the NOx, nitrous oxide or $\mathrm{N}_{2} \mathrm{O}$, is a greenhouse gas. It accumulates in the atmosphere with other greenhouse gasses causing a gradual rise in the earth's temperature. This will lead to increased risks to human health, a rise in the sea level, and other adverse changes to plant and animal habitat. In the air, NOx reacts readily with common organic chemicals and even ozone, to form a wide variety of toxic products, some of which may cause biological mutations. Examples of these chemicals include the nitrate radical, nitroarenes, and nitrosamines. Nitrate particles and nitrogen dioxide can block the transmission of light, reducing visibility in urban areas and on a regional scale in US national parks (EPA, 2008). 
Since the 1970s, EPA has required motor vehicle manufacturers to reduce millions of tons of NOx from their products, resulting in noteworthy improvements to public health. As a result of the Tier 2 program, all cars, SUVs, pickups, and vans will be 77-95 percent cleaner by 2009 . The requirements are phased in, starting in 2004 . The program will also result in cleaner-burning gasoline that contains 90 percent less sulfur. Through its clean diesel truck and buses program, EPA reduced the level of sulfur in highway diesel fuel by 97 percent starting in 2006, and will reduce NOx emissions by over $90 \%$. The Clean Air Nonroad Diesel Rule will cut nonroad diesel vehicle exhaust emissions by more than 90 percent and fuel sulfur levels by 99 percent, beginning in 2007 . To help reduce acid rain, EPA devised a two-phased strategy to cut NOx emissions from coalfired power plants. The first phase, finalized in a rulemaking in 1995, aimed to reduce NOx emissions by over 400,000 tons per year between 1996 and 1999. The second phase began in 2000, and aimed to reduce NOx emissions by over 2 million tons per year. The second phase reduction goal has been surpassed, in part due to additional state-initiated NOx reductions in the Northeast. The Clean Air Act requires states to reduce groundlevel ozone. Since NOx and ozone can be transported long distances, the Act also requires "upwind" states to implement programs that will help "downwind" states meet the ozone standards. EPA issued a rule in 1998 that requires 21 states and the District of Columbia to revise their Implementation Plans to further reduce NOx emissions by taking advantage of newer, cleaner control strategies. The rule does not mandate how the reductions are to be achieved, but gives each affected state a NOx emission target. States have flexibility in determining how to reduce emissions. The goal of this rule is to reduce 
total emissions of NOx by 1 million tons in the affected states by 2007 . Reductions in most states were required to begin in 2004 (EPA, 2008).

\subsubsection{Sulfur dioxide}

$\mathrm{SO}_{2}$ belongs to the family of sulfur oxide gases ( $\mathrm{SOx}$ ). These gases dissolve easily in water. Sulfur is found in all raw materials, including crude oil, coal, and ore that contains common metals like aluminum, copper, zinc, lead, and iron. SOx gases are formed when fuel containing sulfur, such as coal and oil, is burned, and when gasoline is extracted from oil, or metals are extracted from ore. $\mathrm{SO}_{2}$ dissolves in water vapor to form acid, and interacts with other gases and particles in the air to form sulfates and other products that can be detrimental to people and their environment. $\mathrm{SO}_{2}$ causes a wide variety of health and environmental impacts because of the way it reacts with other substances in the air. Particularly sensitive groups include people with asthma who are active outdoors and children, the elderly, and people with heart or lung disease. Peak levels of $\mathrm{SO}_{2}$ in the air can cause temporary breathing difficulty for people with asthma who are active outdoors. Longer-term exposures to high levels of $\mathrm{SO}_{2}$ gas and particles cause respiratory illness and aggravate existing heart disease. $\mathrm{SO}_{2}$ reacts with other chemicals in the air to form tiny sulfate particles. When these are breathed, they gather in the lungs and are associated with increased respiratory symptoms and disease, difficulty in breathing, and premature death. Haze occurs when light is scattered or absorbed by particles and gases in the air. Sulfate particles are the major cause of reduced visibility in many parts of the U.S., including national parks. $\mathrm{SO}_{2}$ and nitrogen oxides react with other substances in the air to form acids, which fall to earth as rain, fog, snow, or dry particles. Some may be carricd by the wind for hundreds of miles. Acid rain harms forests and 
crops, changes the makeup of soil, and makes lakes and streams acidic and unsuitable for fish. Continued exposure over a long time changes the natural variety of plants and animals in an ecosystem. $\mathrm{SO}_{2}$ accelerates the decay of building materials and paints, including precious monuments, statues, and sculptures that are part of any nation's cultural heritage.

Reducing emissions of $\mathrm{SO}_{2}$ remains a crucial element of EPA's strategy for cleaner air. Meeting EPA's national health-based air quality standards is an important stage towards ensuring the air is safe to breathe. To meet the standards, EPA, states, tribes, and local governments work as partners to decrease emissions of $\mathrm{SO}_{2}$ through several control programs. Meeting the national ambient air quality standards for sulfur dioxide entails working with state and local governments to ensure that the levels of $\mathrm{SO}_{2}$ in the air are not unhealthy. Breathing $\mathrm{SO}_{2}$ can cause respiratory illness and aggravate existing heart and lung diseases. $\mathrm{SO}_{2}$ is a primary contributor to the formation of acid rain, which is associated with acidification of soils, lakes, and streams, and accelerated corrosion of buildings and monuments. To help reduce acid rain, EPA is implementing a program to reduce releases of $\mathrm{SO}_{2}$ and other pollutants from coal-fired power plants. The first phase began in 1995 for $\mathrm{SO}_{2}$ and targets the largest and highest emitting power plants. The second phase (started in 2000) sets tighter restrictions on smaller coal-, gas-, and oil-fired plants. This program will reduce annual $\mathrm{SO}_{2}$ emissions by 10 million tons (almost half the 1980 level) between 1980 and 2010. Particulate matter can be formed from direct sources (like diesel exhaust or smoke), but can also be formed through chemical reactions. Emissions of $\mathrm{SO}_{2}$ can be chemically transformed into ammonium sulfates, which are very tiny particles that can be carried by winds hundred of miles. These small 
particles have been shown to cause a series of health problems for asthmatics, the elderly, and other people with pre-existing respiratory problems. These same small particles are also a chief pollutant that reduce visibility across large areas of the country, particularly national parks that are known for their scenic vistas. The nation has made great steps forward in meeting the first goal of meeting the national air quality standards. Levels of $\mathrm{SO}_{2}$ in the air have decreased over the past 20 years. These reductions over time were achieved by installing pollution control equipment at coal-fired power plants, decreasing pollution from industrial processing facilities, reducing the average sulfur content of fuels burned, and using cleaner fuels like natural gas for residential and commercial heat. However, further reductions of $\mathrm{SO}_{2}$ and other pollutants will be required to solve the particulate matter and acid rain problems (EPA, 2008).

\subsubsection{Lead}

In the past, motor vehicles were the main contributor of lead emissions to the air. As a result of EPA's regulatory efforts to reduce lead in gasoline, air emissions of lead from the transportation sector, and particularly the automotive sector, have greatly declined over the past two decades. Today industrial processes, primarily metals processing, are the major source of lead emissions to the air. The highest air concentrations of lead are usually found near lead smelters. Other stationary sources are waste incinerators, utilities, and lead-acid battery manufacturers. In addition to exposure to lead in air, other major exposure channels include intake of lead in drinking water and lead-contaminated food as well as incidental ingestion of lead-contaminated soil and dust. Lead-based paint remains a major exposure pathway in older homes. Once taken into the body, lead distributes throughout the body in the blood and is amassed in the bones. 
Depending on the level of exposure, lead can adversely affect the nervous system, kidney function, immune system, reproductive and developmental systems and the cardiovascular system. Lead exposure also affects the oxygen carrying capacity of the blood. The lead effects most commonly encountered in current populations are neurological effects in children and cardiovascular effects (e.g., high blood pressure and heart disease) in adults. Infants and young children are especially sensitive to even low levels of lead, which may contribute to behavioral problems, learning deficits and lowered IQ.

Lead does not decay easily in the environment and accumulates in soils and sediments through deposition from air sources, direct discharge of waste streams to water bodies, mining, and erosion. Ecosystems near point sources of lead demonstrate a wide range of harmful effects including losses in biodiversity, changes in community composition, decreased growth and reproductive rates in plants and animals, and neurological effects in vertebrates. EPA Strategy for Reducing Lead Exposure, the Agency focused primarily on regulatory and remedial clean-up efforts to reduce $\mathrm{Pb}$ exposure from a variety of non-air sources that posed more extensive public health risks, as well as other actions to reduce air emissions.

\subsubsection{National Ambient Air Quality Standards (NAAOS)}

The Clean Air Act, which was last amended in 1990, requires EPA to set National Ambient Air Quality Standards (40 CFR part 50) for pollutants considered harmful to public health and the environment. The Clean Air Act established two types of national air quality standards. "Primary standards" set limits to protect public health, including the health of sensitive populations such as asthmatics, children, and the elderly. "Secondary 
standards" set limits to protect public welfare, including protection against decreased visibility, damage to animals, crops, vegetation, and buildings.

The EPA Office of Air Quality Planning and Standards (OAQPS) has set National Ambient Air Quality Standards for six principal pollutants, which are called "criteria" pollutants. They are listed below. Units of measure for the standards are parts per million $(\mathrm{ppm})$ by volume, milligrams per cubic meter of air $\left(\mathrm{mg} / \mathrm{m}^{3}\right)$, and micrograms per cubic meter of air $\left(\mu \mathrm{g} / \mathrm{m}^{3}\right)$.

Table 2.1 National Ambient Air Quality Standards

\begin{tabular}{|c|c|c|c|c|}
\hline \multirow{2}{*}{ Pollutant } & \multicolumn{2}{|c|}{ Primary Standards } & \multicolumn{2}{|c|}{ Secondary Standards } \\
\hline & Level & Averaging Time & Level & $\begin{array}{l}\text { Averaging } \\
\text { Time }\end{array}$ \\
\hline \multirow[t]{2}{*}{$\begin{array}{l}\text { Carbon } \\
\text { Monoxide }\end{array}$} & $\begin{array}{l}9 \mathrm{ppm} \\
\left(10 \mathrm{mg} / \mathrm{m}^{3}\right)\end{array}$ & 8-hour ${ }^{(1)}$ & \multirow{2}{*}{\multicolumn{2}{|c|}{ None }} \\
\hline & $\begin{array}{l}35 \mathrm{ppm} \\
\left(40 \mathrm{mg} / \mathrm{m}^{3}\right)\end{array}$ & 1 -hour ${ }^{(1)}$ & & \\
\hline Lead & $1.5 \mu \mathrm{g} / \mathrm{m}^{3}$ & Quarterly Average & \multicolumn{2}{|c|}{ Same as Primary } \\
\hline $\begin{array}{l}\text { Nitrogen } \\
\text { Dioxide }\end{array}$ & $\begin{array}{l}0.053 \mathrm{ppm} \\
\left(100 \mu \mathrm{g} / \mathrm{m}^{3}\right)\end{array}$ & $\begin{array}{l}\text { Annual } \\
\text { (Arithmetic Mean) }\end{array}$ & \multicolumn{2}{|c|}{ Same as Primary } \\
\hline $\begin{array}{l}\text { Particulate } \\
\text { Matter } \\
\left(\mathrm{PM}_{10}\right)\end{array}$ & $150 \mu \mathrm{g} / \mathrm{m}^{3}$ & 24-hour $(2)$ & \multicolumn{2}{|c|}{ Same as Primary } \\
\hline \multirow{2}{*}{$\begin{array}{l}\text { Particulate } \\
\text { Matter } \\
\left(\mathrm{PM}_{2.5}\right)\end{array}$} & $15.0 \mu \mathrm{g} / \mathrm{m}^{3}$ & $\begin{array}{l}\text { Annual }^{(3)} \\
\text { (Arithmetic Mean) }^{-}\end{array}$ & \multicolumn{2}{|c|}{ Same as Primary } \\
\hline & $35 \mu \mathrm{g} / \mathrm{m}^{3}$ & 24-hour ${ }^{(4)}$ & \multicolumn{2}{|c|}{ Same as Primary } \\
\hline \multirow[t]{3}{*}{ Ozone } & $\begin{array}{l}0.075 \text { ppm }(2008 \\
\text { std })\end{array}$ & 8 -hour ${ }^{(5)}$ & \multicolumn{2}{|c|}{ Same as Primary } \\
\hline & $\begin{array}{l}0.08 \mathrm{ppm}(1997 \\
\text { std) }\end{array}$ & 8 -hour $^{(6)}$ & \multicolumn{2}{|c|}{ Same as Primary } \\
\hline & $0.12 \mathrm{ppm}$ & $\begin{array}{l}1 \text {-hour }^{(7)} \\
\text { (Applies only in limited } \\
\text { areas) }\end{array}$ & \multicolumn{2}{|c|}{ Same as Primary } \\
\hline \multirow[t]{2}{*}{$\begin{array}{l}\text { Sulfur } \\
\text { Dioxide }\end{array}$} & $0.03 \mathrm{ppm}$ & $\begin{array}{l}\text { Annual } \\
\text { (Arithmetic Mean) }\end{array}$ & \multirow{2}{*}{$\begin{array}{l}0.5 \mathrm{ppm} \\
(1300 \\
\left.\mu \mathrm{g} / \mathrm{m}^{3}\right)\end{array}$} & \multirow[t]{2}{*}{3 -hour ${ }^{(1)}$} \\
\hline & $0.14 \mathrm{ppm}$ & 24-hour ${ }^{(1)}$ & & \\
\hline
\end{tabular}


(1) Not to be exceeded more than once per year.

${ }^{(2)}$ Not to be exceeded more than once per year on average over 3 years.

(3) To attain this standard, the 3-year average of the weighted annual mean PM2.5 concentrations from single or multiple community-oriented monitors must not exceed $15.0 \mu \mathrm{g} / \mathrm{m} 3$.

(4) To attain this standard, the 3-year average of the 98th percentile of 24 -hour concentrations at each population-oriented monitor within an area must not exceed 35 $\mu \mathrm{g} / \mathrm{m} 3$ (effective December 17, 2006).

5) To attain this standard, the 3-year average of the fourth-highest daily maximum 8-hour average ozone concentrations measured at each monitor within an area over each year must not exceed $0.075 \mathrm{ppm}$. (effective May 27, 2008)

(6) (a) To attain this standard, the 3-year average of the fourth-highest daily maximum 8hour average ozone concentrations measured at each monitor within an area over each year must not exceed $0.08 \mathrm{ppm}$.

(b) The 1997 standard - and the implementation rules for that standard-will remain in place for implementation purposes as EPA undertakes rulemaking to address the transition from the 1997 ozone standard to the 2008 ozone standard.

(7) (a) The standard is attained when the expected number of days per calendar year with maximum hourly average concentrations above $0.12 \mathrm{ppm}$ is $\leq 1$.

(b) As of June 15, 2005 EPA revoked the 1-hour ozone standard in all areas except the 8-hour ozone nonattainment Early Action Compact (EAC) Areas.

\subsubsection{How the Clean Air Act Reduces Air Pollution Such as Particle Pollution}

\section{and Ground-level Ozone}

First, EPA works with state governors and tribal government leaders to identify "nonattainment" areas where the air does not meet allowable limits for a common air pollutant. States and tribes usually do much of the planning for cleaning up common air pollutants. They develop plans, called State/Tribal Implementation Plans, to reduce air pollutants to allowable levels. Then they use a permit system as part of their plan to make sure power plants, factories, and other pollution sources meet their goals to clean up the air.

The Clean Air Act requirements are comprehensive and cover a variety of pollution sources and clean-up methods to reduce common air pollutants. Many of the clean-up 
requirements for particle pollution and ground-level ozone involve large industrial sources (power plants, chemical producers, and petroleum refineries), as well as motor vehicles (cars, trucks, and buses). Also, in nonattainment areas, controls are generally required for smaller pollution sources, such as gasoline stations and paint shops.

\subsection{Transportation and Air Quality}

In response to the Clean Air Act (CAA), the U.S. Environmental Protection Agency (EPA) established National Ambient Air Quality Standards (NAAQS) for various pollutants - known as criteria pollutants - that adversely affect human health and welfare. The three major transportation-related criteria pollutants are as follows:

1. Ozone $\left(\mathrm{O}_{3}\right)$ and its precursors, volatile organic compounds (VOC) and oxides of nitrogen $\left(\mathrm{NO}_{\mathrm{x}}\right)$

2. Particulate matter (PM)

3. Carbon monoxide

Other criteria pollutants include sulfur dioxide $\left(\mathrm{SO}_{2}\right)$, nitrogen dioxide $\left(\mathrm{NO}_{2}\right)$, and lead $(\mathrm{Pb})$. In the past, motor vehicles were a major source of lead emissions that were virtually eliminated as leaded gasoline was phased out. Although not criteria air pollutants, toxic air pollutants/air toxics, are the pollutants that cause or may cause cancer or other serious health effects, such as reproductive effects or birth defects. Air toxics may also cause adverse environmental and ecological effects. The CAA identifies 188 air toxics. The EPA has identified 21 pollutants as mobile source air toxics, including diesel particulate matter, benzene, and other organic materials and metals. 
Fuel combustion by motor vehicles and other sources releases carbon dioxide $\left(\mathrm{CO}_{2}\right)$, which is a "greenhouse gas" that traps heat within the earth's atmosphere. $\mathrm{CO}_{2}$ is not directly dangerous to human health and is not regulated under the CAA.

Significant progress has been made in reducing criteria pollutant emissions from motor vehicles and improving air quality since the 1970 s, even as vehicle travel has increased rapidly. The air is noticeably cleaner than in 1970 , and total criteria-pollutant emissions from motor vehicles are less than they were in 1970 despite a near tripling of vehicle miles of travel. With the reduction in criteria pollutants, many air toxics have also been reduced.

Still, challenges remain. In 1997, EPA issued revised standards for ozone and particulate matter that reflect improved understanding of the health effects of these pollutants. Based on monitoring data, approximately 146 million people in the United States reside in counties that do not meet the air-quality standards for at least one NAAQS pollutant.

\subsubsection{Carbon-monoxide}

Although one cannot see or smell carbon monoxide, this poisonous gas is a major air pollutant in many American cities. Carbon monoxide forms when carbon in fuel doesn't burn completely (incomplete combustion). The main source of carbon monoxide in our air is vehicle emissions. As much as 95 percent of the carbon monoxide in typical U.S. cities comes from mobile sources, according to EPA studies. 


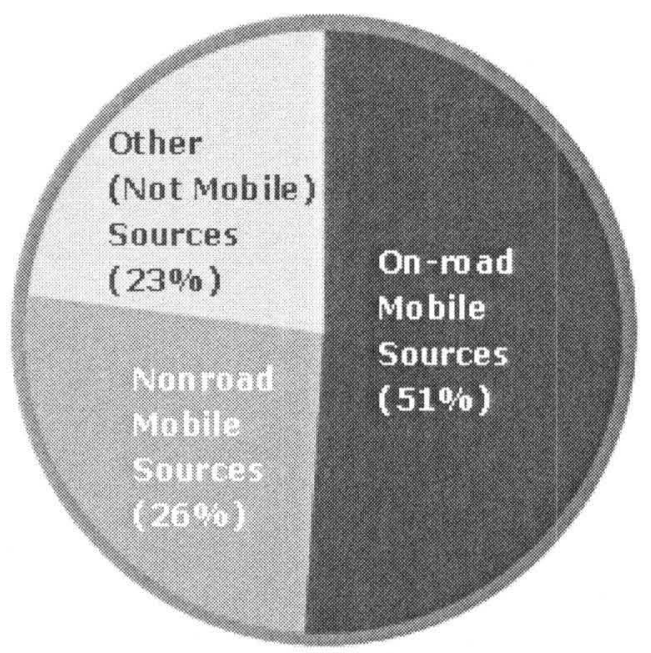

[Source: EPA, 2008]

Figure 2.1

1999 National Emissions by Source: Carbon Monoxide

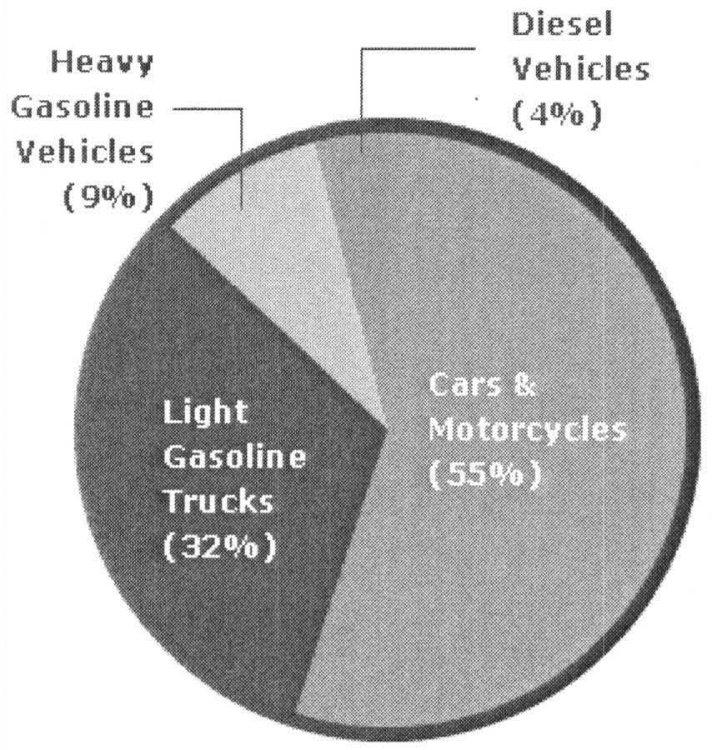

[Source: EPA, 2008]

Figure 2.2

1999 National Emissions by Source: Carbon Monoxide On-Road Mobile Sources 
It is obvious from Figure $1.1 \& 1.2$, that major contribution $(51 \%)$ to the ambient air of Carbon-monoxide comes from on-road Mobile Sources and Cars \& Motorcycles are responsible for $55 \%$ out of that amount. If pollution from Cars \& Motorcycles could be curtailed, significant reduction in the concentration of $\mathrm{CO}$ in ambient air would follow automatically.

\subsubsection{Hydrocarbons}

Hydrocarbons are a precursor to ground-level ozone, a serious air pollutant in cities across the United States. A key component of smog, ground-level ozone is formed by reactions involving hydrocarbons and nitrogen oxides in the presence of sunlight.

Hydrocarbon emissions result from incomplete fuel combustion and from fuel evaporation. Today's cars are equipped with emission controls designed to reduce both exhaust and evaporative hydrocarbon emissions.

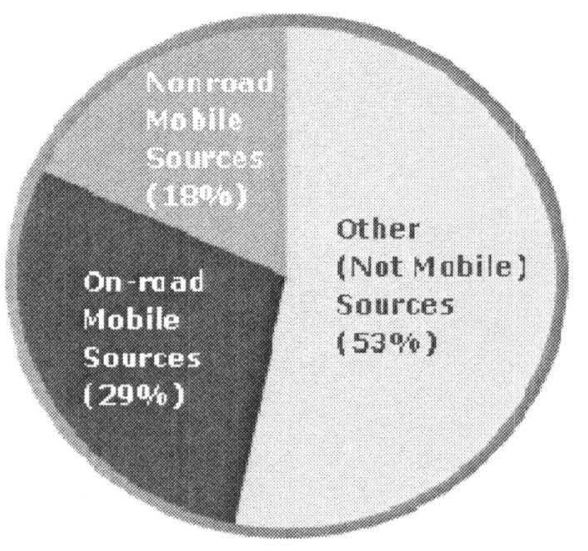

[Source: EPA, 2008]

Figure 2.3

1999 National Emissions by Source: Hydrocarbons 
Though the major portion of Hydrocarbons to ambient air comes from other sources such as Gasoline and Diesel equipments as can be seen from Figure 2.3, On-Road mobile sources also consitute a significant part. From Figure 2.4, it is evident that Motorcycles and cars, once again, are major contributor out of On-Road mobile sources to the Hydrocarbon pollution in ambient air.

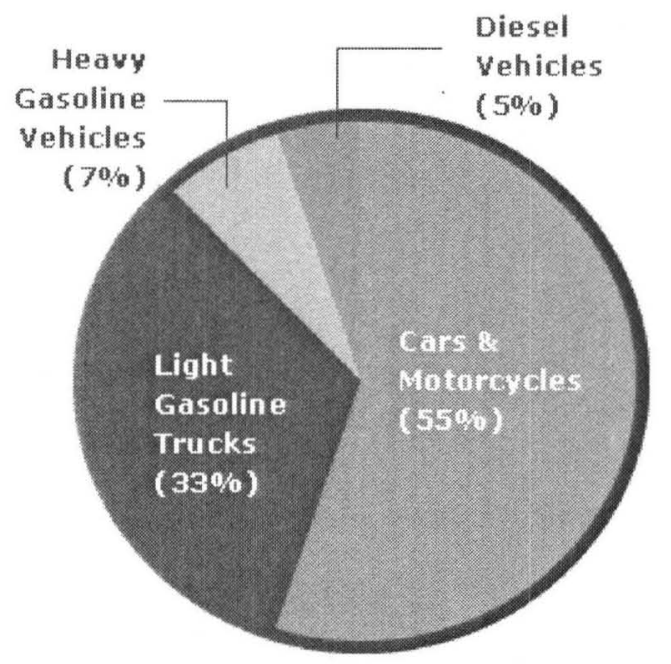

[Source: EPA, 2008]

Figure 2.4

1999 National Emissions by Source: Hydrocarbons On-Road Mobile Sources 


\subsubsection{Nitrogen Oxides}

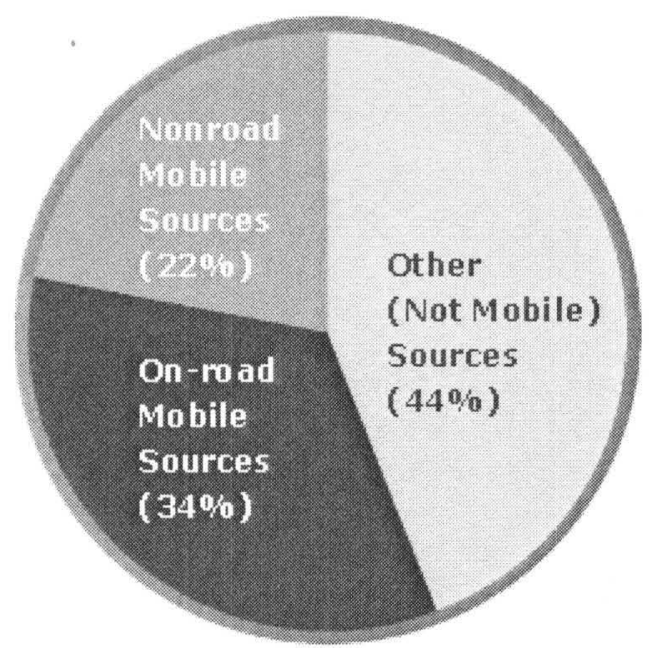

[Source: EPA, 2008]

Figure 2.5

1999 National Emissions by Source: Nitrogen Oxides

Nitrogen oxides form when fuel burns at high temperatures, such as in motor

vehicle engines. Mobile sources are responsible for more than half of all nitrogen oxide emissions in the United States. Both on-road and nonroad mobile sources are major nitrogen oxide polluters. 


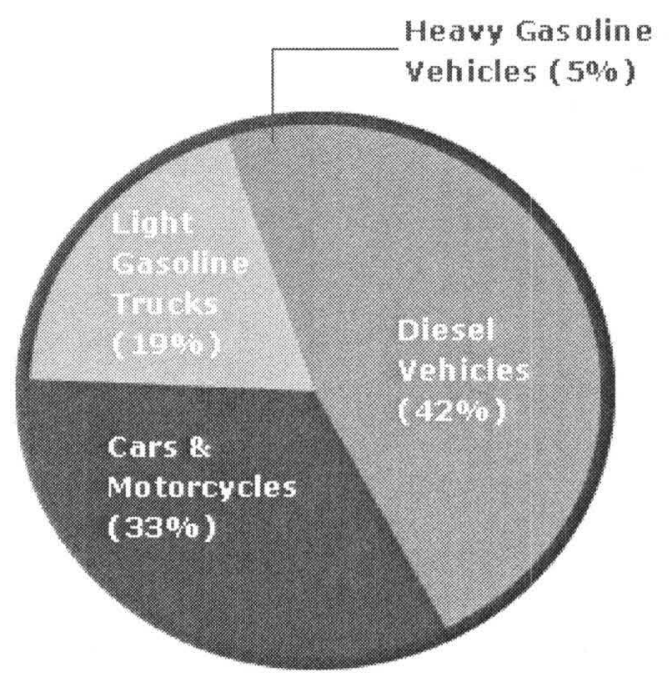

[Source: EPA, 2008]

Figure 2.6

1999 National Emissions by Source: Nitrogen Oxides

On-Road Mobile Sources

From Figure $2.5 \& 2.6$, it is evident that on-road mobile vehicles inject a significant portion of Nitrogen Oxide into the atmosphere and Diesel vehicles are the biggest source of these.

\subsection{ITS Application Overview}

ITS improve transportation safety and mobility and enhance productivity through the use of advanced information and communications technologies. Intelligent transportation systems (ITS) encompass a broad range of wireless and wire line communications-based information and electronics technologies. When integrated into the transportation system's infrastructure, and in vehicles themselves, these technologies relieve congestion, improve safety and enhance American productivity. ITS is made up of 16 types of technology based systems. These systems are divided into intelligent 
infrastructure systems and intelligent vehicle systems (Research and Innovative Technology Administration (RITA), 2008).

\subsubsection{Arterial Management}

Arterial Management, through ITS, consists of Surveillance, Traffic Control, Lane Management, Parking Management, Information Dissemination, and Enforcement as can be seen in Figure 2.7.

\subsubsection{Surveillance}

Many of the services possible through arterial management systems are enabled by traffic surveillance and detection technologies, such as sensors or cameras, monitoring traffic flow. The surveillance and detection technologies used to monitor traffic flow in support of ITS applications are also used to monitor key transportation facilities or infrastructure for security purposes (ITS, America, 2008; RITA, 2008).

\subsubsection{Traffic Control}

Traffic control measures on arterials optimize travel speeds and provide transit signal priority and signal preemption for emergency vehicles. These also improve safety of bicyclists and pedestrians and smooth traffic flow during special events (RITA, 2008; ITS America, 2008). 


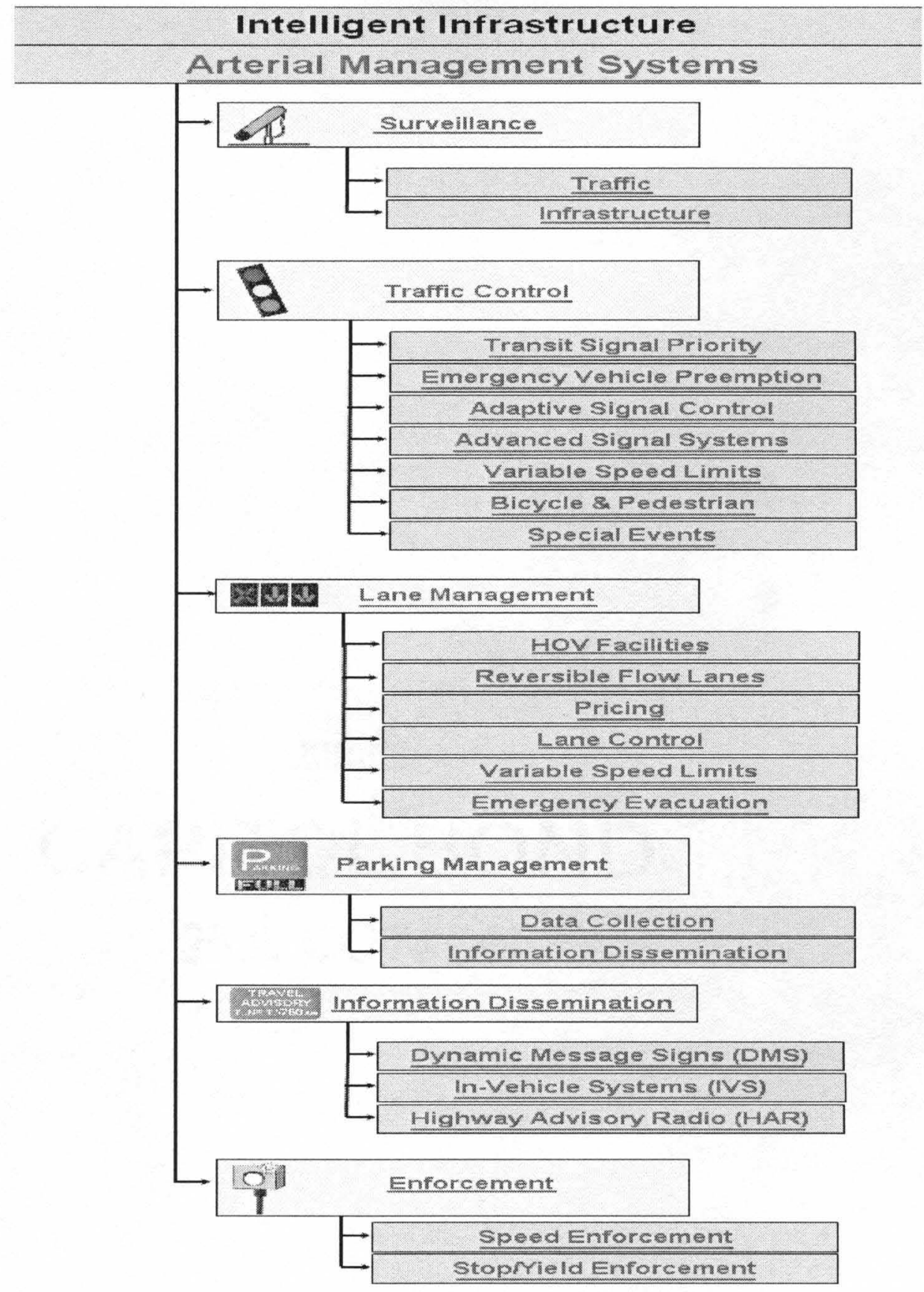

[Source: RITA, 2008]

Figure 2.7

Arterial Management Systems 


\subsubsection{Lane Management}

Lane management applications can promote the most effective use of available capacity during emergency evacuations and incidents, construction and a variety of other traffic and/or weather conditions (RITA, 2008).

\subsubsection{Parking Management}

Parking management systems with information dissemination capabilities, most commonly deployed in urban centers or at modal transfer points such as airports, monitor the availability of parking and disseminate the information to drivers, reducing traveler frustration and congestion associated with searching for parking (ITS, Europe, 2008; RITA, 2008).

\subsubsection{Information Dissemination}

Advanced communications have improved the dissemination of information to the traveling public. Motorists are now able to receive relevant information on locationspecific traffic conditions in a number of ways, including dynamic message signs (DMS), highway advisory radio (HAR), and in-vehicle signing, or specialized information transmitted to individual vehicles (ITS America, 2008; RITA, 2008).

\subsubsection{Enforcement}

Automated enforcement systems, such as speed enforcement and stop/yield enforcement, improve safety and reduce aggressive driving. These systems also assist in the enforcement of traffic signal and speed compliance (ITS America, 2008; RITA, 2008). 


\subsubsection{Freeway Management Systems}

Freeway Management Systems, through ITS, consists of Surveillance, Ramp

Control, Lane Management, Special Event Transportation Management, Information

Dissemination, and Enforcement as can be seen in Figure 2.8.

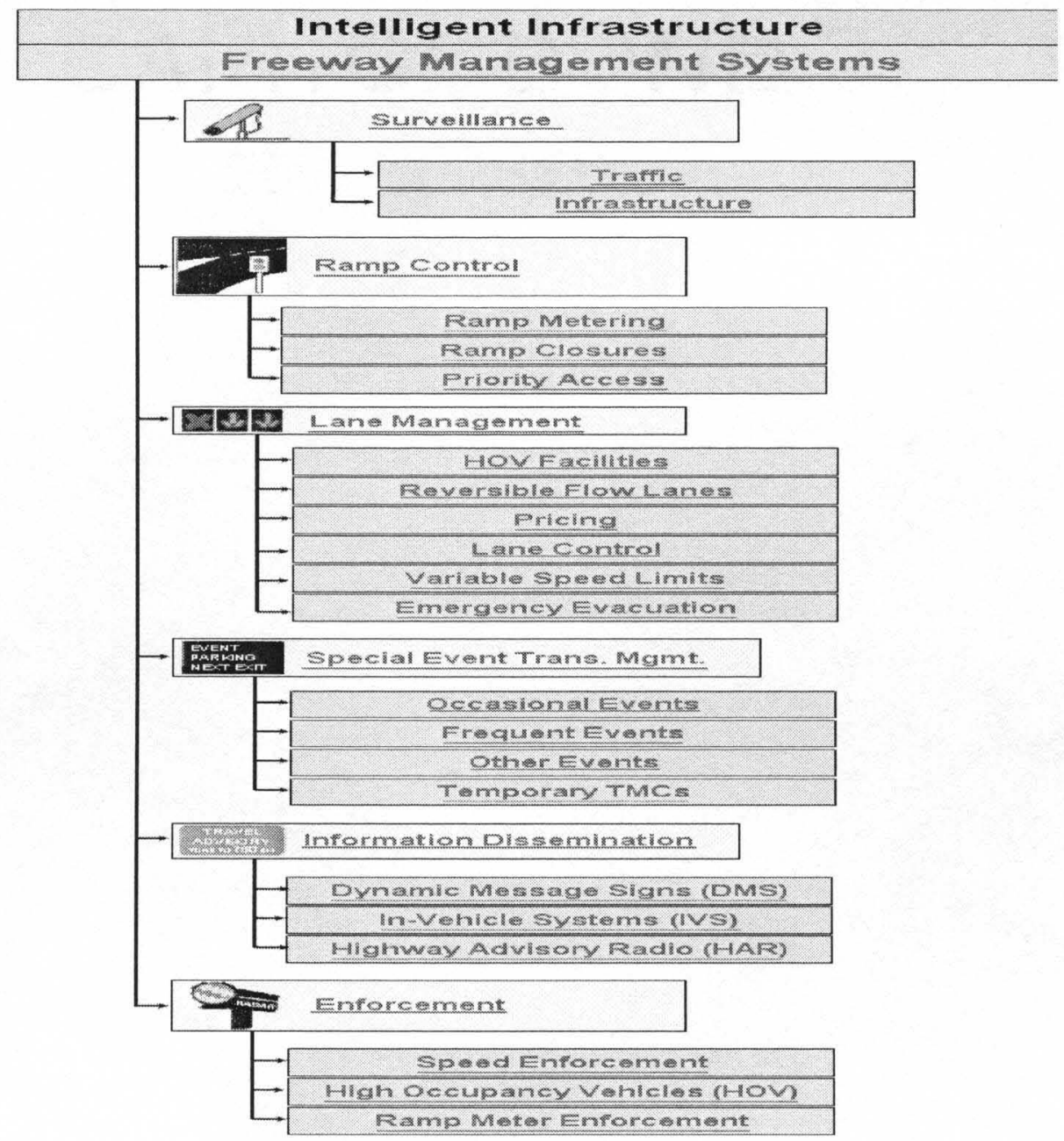

[Source: RITA, 2008]

Figure 2.8

Freeway Management Systems 


\subsubsection{Surveillance}

Traffic surveillance systems use detectors and video equipment to support the most advanced freeway management systems. These sensors can also be used to monitor critical transportation infrastructure for security purposes (ITS America, 2008; RITA, 2008).

\subsubsection{Ramp Control}

Traffic control measures on freeway entrance ramps, such as ramp meters, can use sensor data to optimize freeway travel speeds. Also these measures reduce ramp meter wait times (ITS America, 2008; RITA, 2008).

\subsubsection{Lane Management}

Lane management applications can promote the most effective use of available capacity on freeways to encourage the use of high-occupancy commute modes. These applications include HOV facilities, reversible flow lanes, pricing, lane control, variable speed limits, and emergency evacuation (ITS America, 2008; RITA, 2008).

\subsubsection{Special Event Transportation Management}

Special event transportation management systems can help control the impact of congestion at stadiums or convention centers. In areas with frequent events, large changeable destination signs or other lane control equipment can be installed. In areas with occasional or one-time events, portable equipment can help smooth traffic flow (ITS America, 2008; RITA, 2008). 


\subsubsection{Information Dissemination}

Advanced communications technologies have enhanced the dissemination of information to the traveling community. Motorists are now capable to receive pertinent information on location-specific traffic conditions in a number of ways, including dynamic message signs (DMS), highway advisory radio (HAR), in-vehicle signing, or specialized information transmitted to individual vehicles (ITS America, 2008; RITA, 2008).

\subsubsection{Enforcement}

Automated enforcement systems, such as speed enforcement, high-occupancy vehicle (HOV) lane enforcement, and ramp meter enforcement, improve safety. These systems also reduce aggressive driving (ITS America, 2008; RITA, 2008).

\subsubsection{Transit Management Systems}

Transit ITS services, as can be seen in Figure 2.9, include surveillance and communications, such as automated vehicle location (AVL) systems, computer-aided dispatch (CAD) systems, and remote vehicle and facility surveillance cameras, which enable transit agencies to improve the operational efficiency, safety, and security of the nation's public transportation systems (ITS, Europe, 2008; RITA, 2008).

\subsubsection{Safety and Security}

Advanced software and communications enable data as well as voice to be transferred between transit management centers and transit vehicles for increased safety and security, improved transit operations, and more efficient fleet operations. Transit management centers can monitor in-vehicle and in-terminal surveillance systems to 
improve quality or service and improve the safety and security of passengers and operators (ITS, Australia, 2008; RITA, 2008).

\subsubsection{Transportation Demand Management}

Transportation demand management service, such as ride sharing/matching, dynamic routing/scheduling, and service coordination, increase public access to transit resources where coverage is limited. These systems reduce bus schedule deviation, increase ridership, and decrease paratransit costs (ITS America, 2008; RITA, 2008).

\subsubsection{Fleet Management}

Fleet management systems improve transit reliability through implementation of automated vehicle location (AVL) and computer aided dispatch (CAD) systems which can reduce passenger wait times. These systems may also be implemented with in-vehicle self-diagnostic equipment to automatically alert maintenance personnel of potential problems (ITS America, 2008; ITS, Europe, 2008; RITA, 2008) 


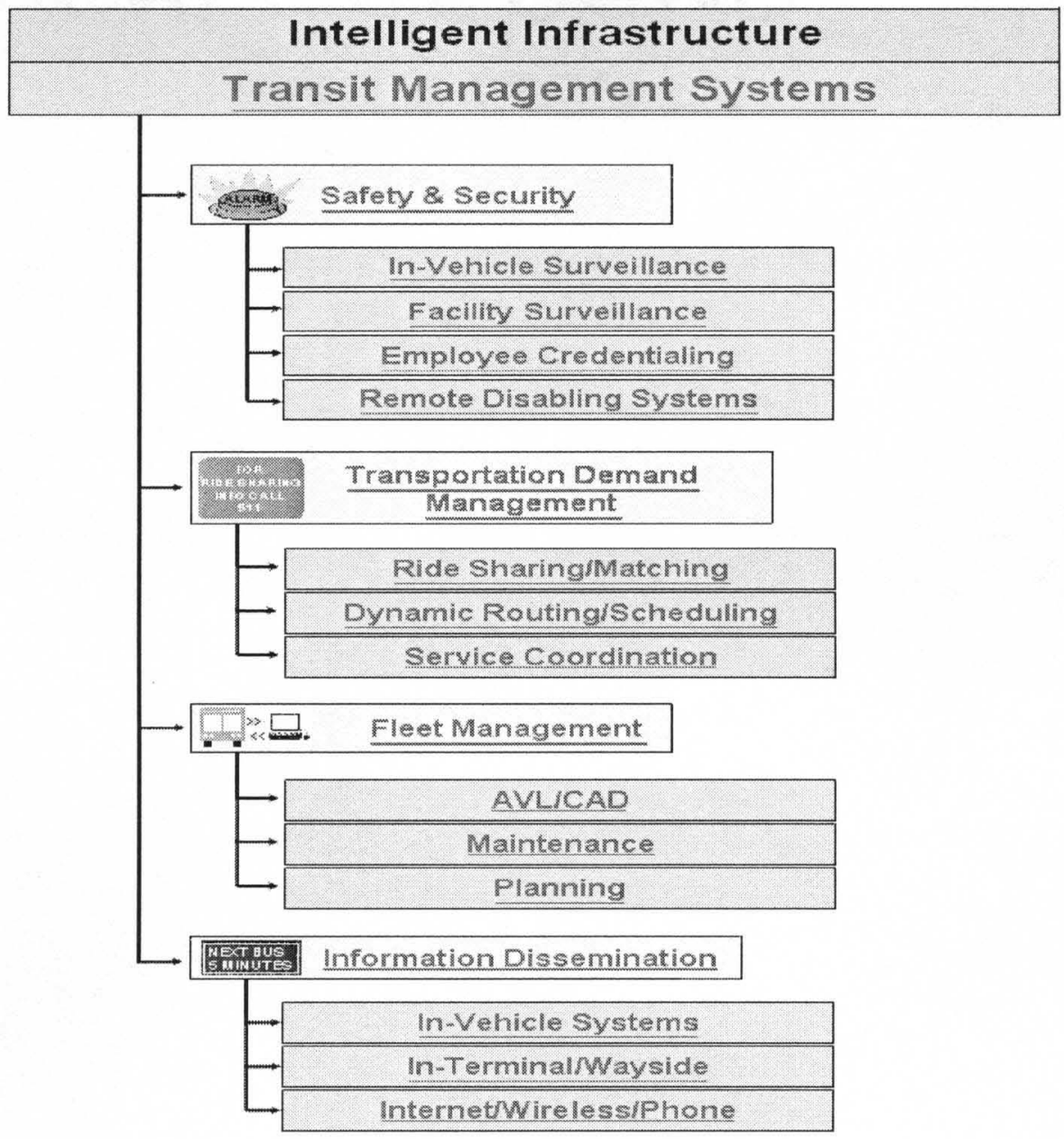

[Source: RITA, 2008]

Figure 2.9

Transit Management Systems

\subsubsection{Information dissemination}

Information dissemination websites allow passengers to confirm scheduling information, improve transfer coordination, and reduce wait times. Electronic transit status information signs at bus stops help passengers manage time, and on-board systems 
such as next-stop audio enunciators help passengers in unfamiliar areas reach their destinations (ITS, Europe, 2008; RITA, 2008).

\subsubsection{Incident Management Systems}

Incident management systems lessen the effects of incident-related congestion by decreasing the time to detect incidents, the time for responding vehicles to arrive, and the time required for traffic to return to normal conditions. As can be seen in Figure 2.10, Incident management systems utilize a variety of surveillance technologies, often shared with freeway and arterial management systems, as well as superior communications and other technologies that facilitate coordinated response to incidents (RITA, 2008; ITS America, 2008).

\subsubsection{Surveillance $\&$ Detection}

An array of surveillance and detection technologies facilitate detect incidents rapidly, including inductive loop or acoustic roadway detectors, and camera systems providing frequent still images or full-motion video. Information from wireless improves 911 systems, mayday, and automated collision notification systems, as well as roadside call boxes can also help incident management system personnel spot incidents quickly (RITA, 2008; ITS America, 2008).

\subsubsection{Mobilization \& Response}

Mobilization and response consists of automated vehicle location and computeraided dispatch systems, and response routing systems. These systems facilitate incident response teams arrive quickly (RITA, 2008; ITS America, 2008). 


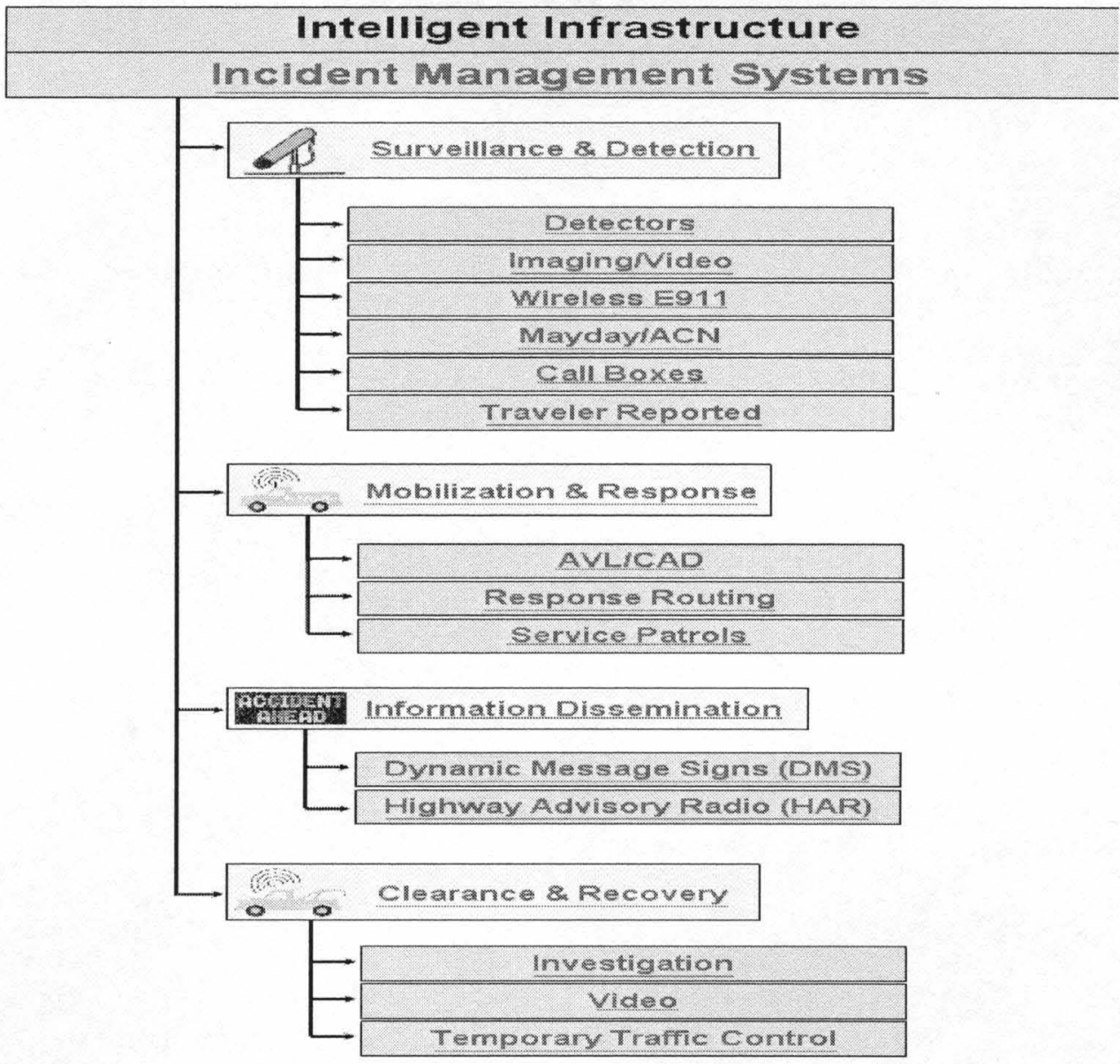

[Source: RITA, 2008]

Figure 2.10

\section{Incident Management Systems}

\subsubsection{Information dissemination}

Information dissemination systems facilitate travelers safely steer around incidents on the roadway. Incident management personnel can directly place incident-related information through Highway Advisory Radio (HAR) and Dynamic Message Signs (DMS). 


\subsubsection{Clearance \& Recovery}

Several technologies are available to accelerate the investigation of incident scenes and record necessary information for later analysis. Temporary traffic control devices assist in making sure the safety of incident responders and provide for the safe travel of vehicles around the incident site (ITS, Australia, 2008; RITA, 2008).

\subsubsection{Emergency Management Systems}

ITS applications in emergency management comprise of hazardous materials management, the deployment of emergency medical services, and large and small-scale emergency response and evacuation operations as can be seen in Figure 2.11.

\subsubsection{Hazardous Materials Management}

ITS applications related with hazardous materials (HAZMAT) consignment can achieve four major functions intended to provide safe and secure transport of hazardous materials by road. Vehicle-mounted hardware provides the capability to track HAZMAT shipments and support notification of management centers when a shipment deviates from its proposed route. Roadside detectors can check for the presence of hazardous shipments in sensitive areas and, if electronic tag information is available on the detected vehicle, corroborate that the shipment is on the proposed route. Driver authentication technology can make sure that the individual operating a HAZMAT vehicle is certified to do so and report operation by unanticipated drivers to public safety entities. ITS also offer assistance to commercial vehicle operations via electronic route planning services, ensuring compliance with HAZMAT shipment restrictions along planned travel routes(RITA, 2008). 


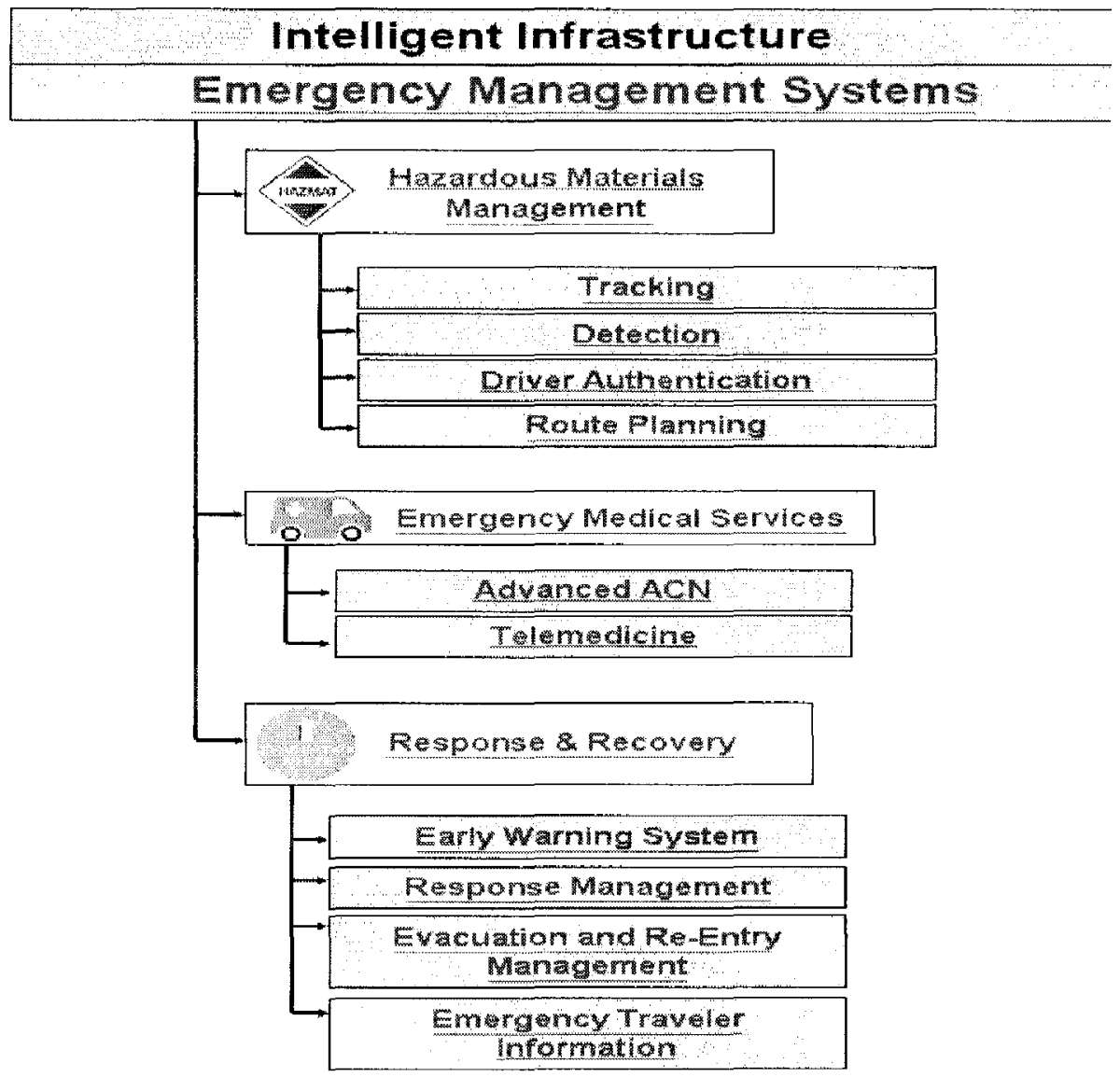

[Source: RITA, 2008]

\section{Figure 2.11}

\section{Emergency Management Systems}

\subsubsection{Emergency Medical Services}

Advanced automated collision notification $(\mathrm{ACN})$ and telemedicine tackle the detection of and response to incidents such as vehicle collisions or other incidents requiring emergency responders. In rural areas, response time for emergency medical services is greater than in urban areas, resulting in fatal consequences for those in want of medical assistance. Advances ACN systems can alert emergency personnel and provide them with valuable information on the crash, including location, crash characteristics, and 
possible appropriate medical information regarding the vehicle occupants. Telemedicine systems provide a connection between responding ambulances and emergency medical facilities, enabling doctors to counsel emergency medical personnel regarding treatment of patients on the way to the hospital (RITA, 2008; ITS America, 2008).

\subsubsection{Response \& Recovery}

The assortment of sensors deployed on the transportation infrastructure can help provide an early warning system to spot large-scale emergencies, including natural disasters and technological and man-made disasters. In the event of a large-scale emergency, ITS applications can help with response management through services such as the tracking of emergency vehicle fleets using automated vehicle location (AVL) technology and two-way communications between emergency vehicles and dispatchers. Evacuation operations often need a synchronized emergency response involving multiple agencies, various emergency centers, and numerous response plans. Integration with traffic and transit management systems enables emergency information to be shared between public and private agencies and the traveling public. This communication and cooperation also enables the use of the variety of ITS information dissemination capabilities to provide emergency traveler information (RITA, 2008; ITS America, 2008).

\subsubsection{Electronic Payment and Pricing}

As it can be seen from Figure 2.12, Electronic Payment and Pricing consists of toll collection, transit fare payment, parking fee payment, multi-use payment and pricing. Electronic payment systems utilize various communication and electronic technologies to facilitate business between travelers and transportation agencies, normally for the 
purpose of paying tolls and transit fares. Pricing refers to charging motorists a fee or toll that varies with the level of demand or with the time of day (RITA, 2008).

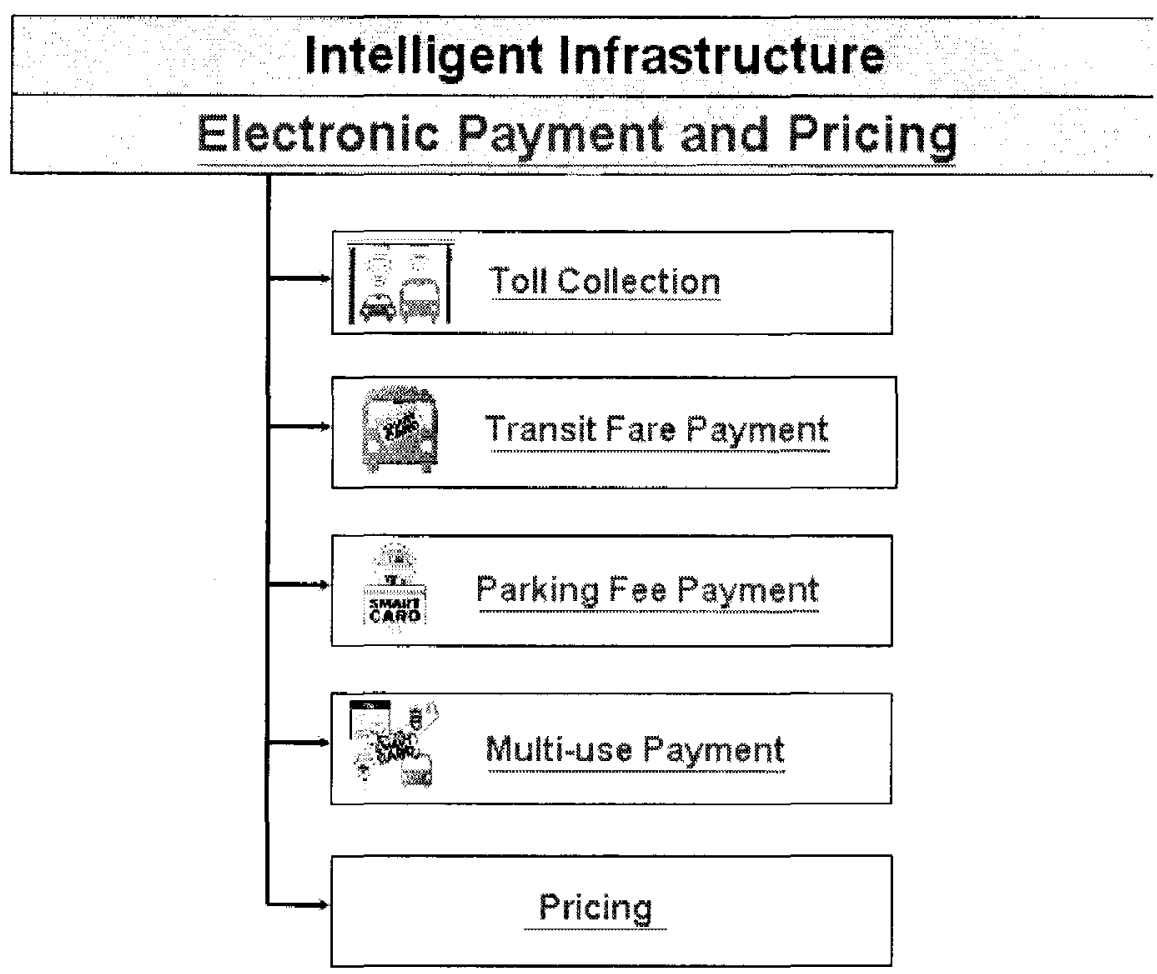

[Source: RITA, 2008]

Figure 2.12

Electronic Payment and Pricing

\subsubsection{Toll Collection}

Electronic toll collection (ETC) supports the collection of payment at toll plazas using automated systems to enhance the operational efficiency and expediency of toll collection. Systems typically consist of vehicle-mounted transponders identified by 
readers located in dedicated and/or mixed-use lanes at toll plazas (RITA, 2008; ITS America, 2008).

\subsubsection{Transit Fare Payment}

Electronic transit fare payment systems, often cnabled by smart card or magnetic stripe technologies, provide increased convenience to customers. These systems make significant cost savings to transportation agencies by increasing the efficiency of money handling processes and improving administrative controls (RITA, 2008; ITS America, 2008).

\subsubsection{Parking Fee Payment}

Electronic parking fee payment systems provide benefits to parking facility operators, simplify payment for customers, and decrease congestion at entrances and exits to parking facilities. These payment systems are enabled by a variety of technologies including magnetic stripe cards, smart cards, in-vehicle transponders, or vehicle-mounted bar-codes (RITA, 2008; ITS America, 2008).

\subsubsection{Multi-use Payment}

Multi-use payment systems facilitate transit payment more easily. Payment for bus,

rail, and other public or private sector goods and services can be made using transit fare cards at terminal gates, or on check-out counters and phone booths of participating merchants located near transit stations. Multi-use systems may also include the capability to pay highway tolls with the same card (RITA, 2008; ITS Europe, 2008).

\subsubsection{Pricing}

Pricing, also known as congestion pricing or value pricing, makes the use of technologies to vary the cost to use a transportation facility or network based on demand 
or the time of day. Pricing strategies include: variable priced lanes, variable tolls on entire roadways or roadway segments, cordon charging, area-wide charging and fast and intertwined regular (FAIR) lanes (RITA, 2008, ITS Europe, 2008).

\subsubsection{Traveler Information}

As shown in Figure 2.13, Traveler Information applications employ a mix of technologies, including Internet websites, telephone hotlines, as well as television and radio, to let users to make more informed decisions regarding trip departures, routes, and mode of travel. Ongoing implementation of the designated 511 telephone number will improve access to traveler information across the country.

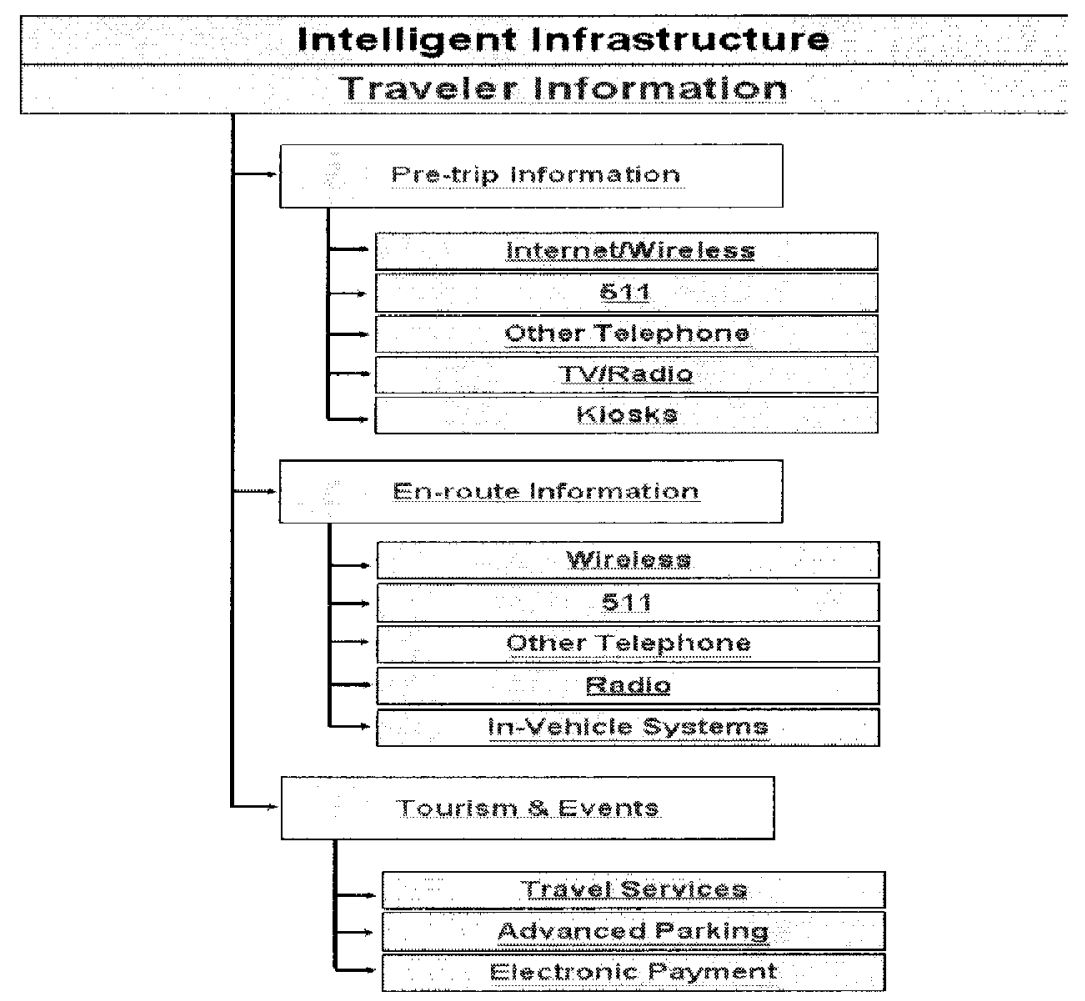

[Source: RITA, 2008]

Figure 2.13

Traveler Information 


\subsubsection{Pre-trip Information}

Pre-trip traveler information provided via internet websites, other wireless devices, 511 telephone numbers, other telephone services, television, radio or kiosks allows users to take a more informed decision for trip departures, routes, and mode of travel. These services greatly reduce traffic demand through the work zone resulting in lowering of average peak delay (RITA, 2008; ITS America, 2008).

\subsubsection{En-route Information}

En-route traveler information is provided through wireless devices, 511 telephone numbers, other telephone services, radio, and in-vehicle signing. These services allow users to make informed decisions regarding alternate routes and expected arrival times (RITA, 2008; ITS America, 2008).

\subsubsection{Tourism and Events}

Tourism and event-related travel information systems cater the needs of travelers in areas alien to them or when traveling to major events such as sporting events or concerts. These services enhance the mobility and traveler convenience. Information provided includes electronic yellow pages as well as transit and parking availability (RITA, 2008; ITS America, 2008).

\subsubsection{Information Management}

ITS information management supports the archiving and retrieval of data generated by other ITS applications and enables ITS applications that use archived information. Decision support systems, predictive information, and performance monitoring are some ITS applications enabled by ITS information management. Also ITS information 
management systems can help in transportation planning, research, and safety management activities (RITA, 2008).

\subsubsection{Data Archiving}

Data archiving is the collection, storage and distribution of ITS data for transportation planning, administration, policy, operation, safety analyses, and research. Data archiving systems use an array of software, database, and electronic data storage technologies (RITA, 2008; ITS America, 2008).

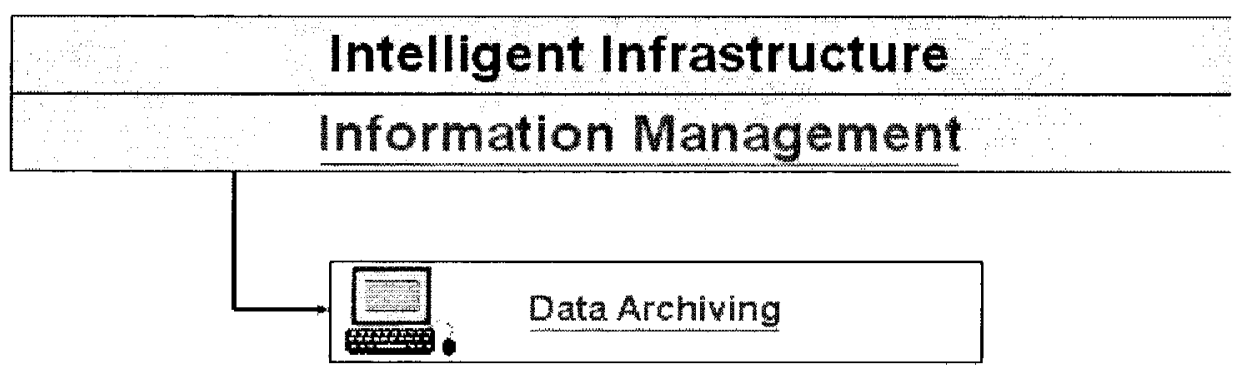

[Source: RITA, 2008]

\section{Figure 2.14}

\section{Information Management}

\subsubsection{Crash Prevention and Safety}

Crash prevention and safety systems identify unsafe conditions and provide warnings to travelers to take action to evade crashes. These systems provide alerts for traffic approaching at dangerous curves, off ramps, restricted overpasses, highway-rail crossings, high-volume intersections, and also provide warnings of the existence of pedestrians, and bicyclists, and even animals on the roadway. Crash prevention and safety systems typically utilize sensors to monitor the speed and characteristics of approaching vehicles and frequently also include environmental sensors to monitor roadway conditions and visibility. These systems may be either permanent or temporary. 
Some systems provide a general warning of the recommended speed for prevailing roadway conditions. Other systems provide a specific warning by taking into account the particular vehicle's characteristics (truck or car) and a calculation of the recommended speed for the particular vehicle based on conditions. In some cases, manual systems are employed, for example where pedestrians or bicyclists manually set the system to provide warnings of their presence to travelers (RITA, 2008).

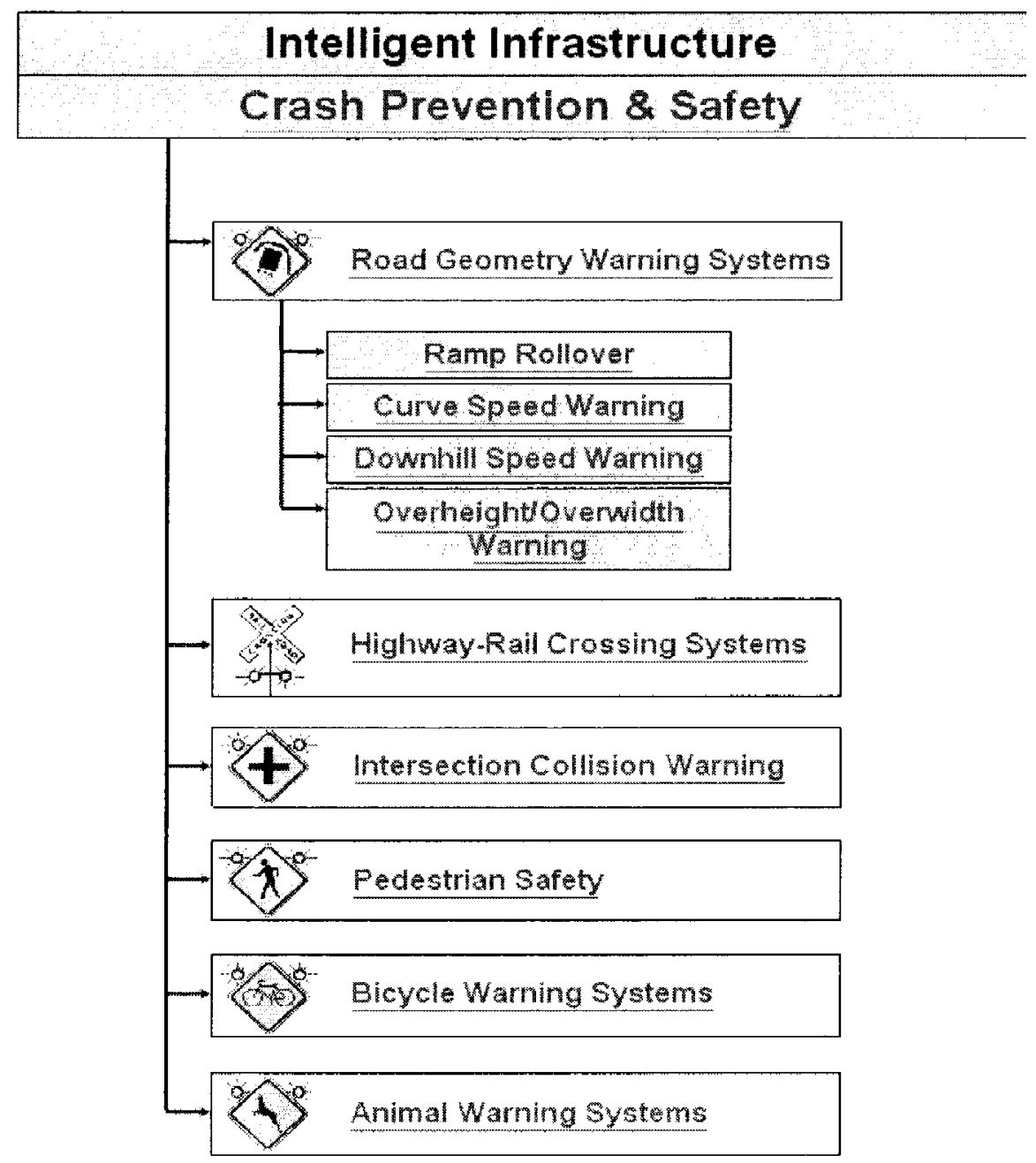

[Source: RITA, 2008]

Figure 2.15

\section{Crash Prevention and Safety}




\subsubsection{Road Geometry Warning Systems}

Road geometry warning systems caution drivers, typically those in commercial trucks and other heavy vehicles, of potentially unsafe conditions that may cause rollovers or other crashes on ramps, curves, or downgrades. Advance curve speed warning and downhill truck speed warning systems reduce truck speed significantly (RITA, 2008; ITS America, 2008).

\subsubsection{Highway Rail Crossing Systems}

Highway rail crossing systems employ detectors, electronic warning signs and automated enforcement technologies to warn roadway traffic of approaching trains. These systems discourage drivers from violating railroad crossing traffic controls (RITA, 2008; ITS Europe, 2008).

\subsubsection{Intersection Collision Warning}

Intersection collision warning systems employ sensors to monitor traffic approaching unsafe intersections. These systems warn vehicles of approaching cross traffic, via roadside or in-vehicle signage (RITA, 2008; ITS Europe, 2008).

\subsubsection{Pedestrian Safety}

Pedestrian safety systems facilitate protect pedestrians by automatically activating in-pavement lighting to alert drivers as pedestrians enter crosswalks. Other systems include 'countdown' pedestrian traffic signals, and pedestrian detectors that extend the "Walk" phase for pedestrians needing more time to cross a street (RITA, 2008; ITS Europe, 2008). 


\subsubsection{Bicycle Warning Systems}

Bicycle warning systems employ detectors and electronic warning signs to recognize bicycle traffic. These systems warn drivers when a cyclist is in an upcoming segment of roadway to improve safety on narrow bridges and tunnels (RITA, 2008, ITS Europe, 2008).

\subsubsection{Animal Warning Systems}

Animal warning systems characteristically use infrared or other detection technologies to identify large animals approaching the roadway, and notify drivers by activating flashers on warning signs located upstream of high frequency crossing areas. These systems may also turn on in-vehicle warning devices (RITA, 2008).

\subsubsection{Roadway Operations \& Maintenance}

ITS applications in operations and maintenance, as shown in Figure 2.16, concentrate on integrated management of maintenance fleets, specialized service vehicles, hazardous road conditions remediation, and work zone mobility and safety. These applications monitor, evaluate, and disseminate roadway and infrastructure data for operational, maintenance, and managerial uses. ITS assist in securing the safety of workers, and travelers in a work zone while helping traffic flow through and around the construction area. This is often achieved through the temporary use of other ITS services, such as elements of traffic management and incident management programs (RITA, 2008).

\subsubsection{Information Dissemination}

Information dissemination technologies can be deployed temporarily, or existing systems can be restructured periodically to provide information on work zones or other 
highway maintenance activities. Examples of these systems include dynamic message signs (DMS), highway advisory radio (HAR), internet websites, wireless devices, and telephone services (RITA, 2008; ITS America, 2008).

\subsubsection{Asset Management}

Many of the services achievable through arterial management systems are enabled by traffic surveillance and detection technologies, such as sensors or cameras, monitoring traffic flow. The surveillance and detection technologies used to monitor traffic flow in support of ITS applications can also be used to monitor key transportation facilities for security purposes (RITA, 2008; ITS America, 2008).

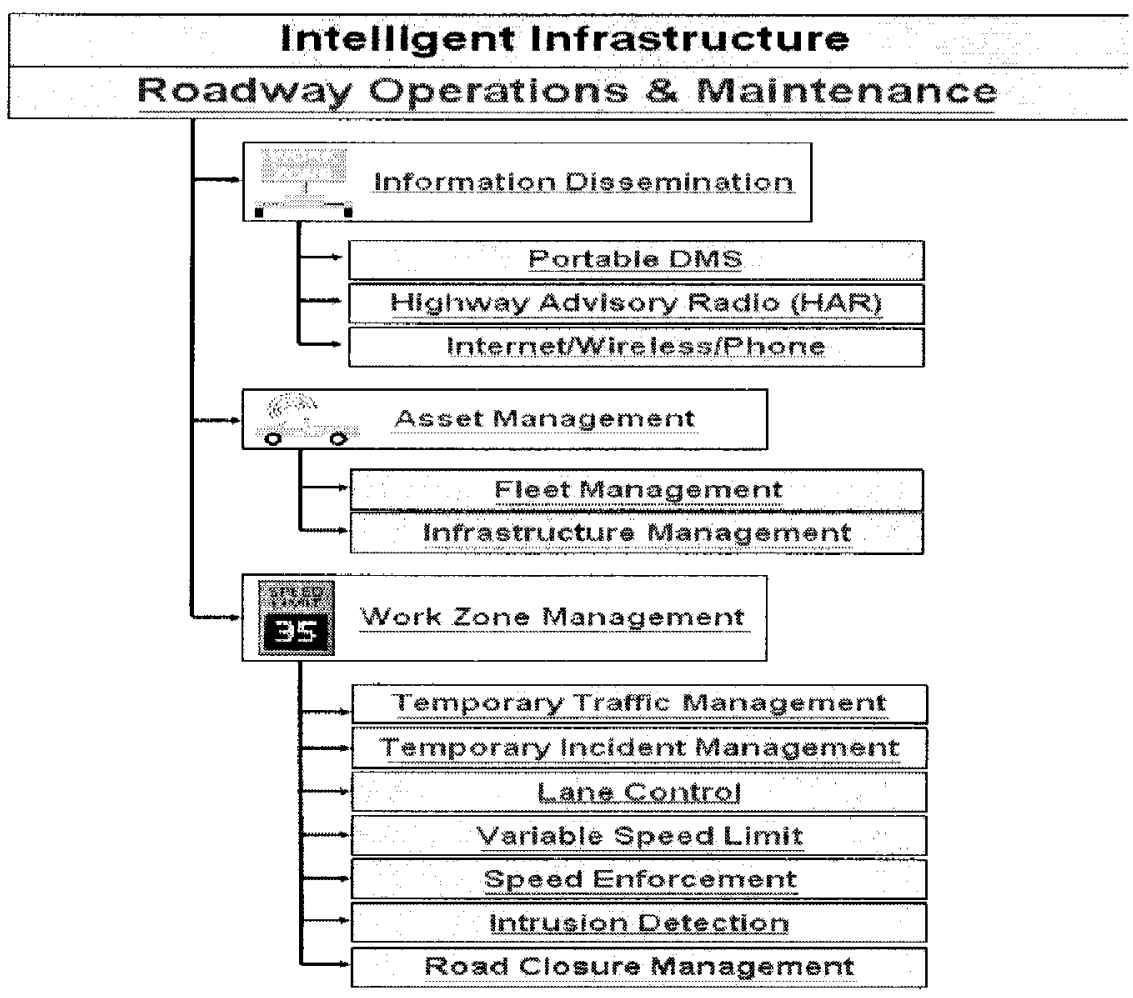

[Source: RITA, 2008]

Figure 2.16

Roadway Operations and Maintenance 


\subsubsection{Work Zone Management}

ITS applications in work zones consist of the temporary implementation of traffic management or incident management capabilities. These temporary systems can be stand-alone implementations or they may supplement existing systems in the area during construction, Other applications for managing work zones include measures to control vehicle speeds and inform travelers of changes in lane configurations or travel times and delays through the work zones. ITS may also be used to control traffic along detour routes during full road closures to facilitate rapid and safe reconstruction projects (RITA, 2008; ITS America, 2008).

\subsubsection{Road Weather Management}

As shown in Figure 2.17, road weather management activities comprise road weather information systems (RWIS), winter maintenance technologies, and coordination of operations within and between state DOTs. ITS applications help with the monitoring and forecasting of roadway and atmospheric conditions, dissemination of weather-related information to travelers, weather-related traffic control measures such as variable speed limits, and both fixed and mobile winter maintenance activities (RITA, 2008; ITS America, 2008).

\subsubsection{Surveillance, Monitoring, \& Prediction}

Surveillance, monitoring, and prediction of weather and roadway conditions facilitate the appropriate management actions to mitigate the impacts of any unfavorable conditions. Low visibility and automated wind warning systems reduce accidents and crashes significantly (RITA, 2008; ITS America, 2008). 


\subsubsection{Information Dissemination}

Information dissemination technologies help road weather managers inform travelers of any unfavorable conditions. DMS about adverse weather conditions can decrease mean driving speeds and reduce accident severity (ITS America, 2008; RITA, 2008).

\subsubsection{Traffic Control}

Traffic control technologies improve traveler safety under poor weather conditions. A variety of technologies let these control measures to be taken quickly in response to devcloping adverse weather (RITA, 2008; ITS America, 2008).

\subsubsection{Response \& Treatment}

A range of ITS applications are being used in the US to support roadway treatments necessary in response to weather events. These applications may provide for automated treatment of the road surface at fixed locations, such as anti-icing systems mounted on bridges in cold climates. They may also increase the efficiency and safety of mobile winter maintenance activities, for example, through automatic vehicle location on snow plows supporting a computer-aided dispatch system (ITS America, 2008; RITA, 2008). 


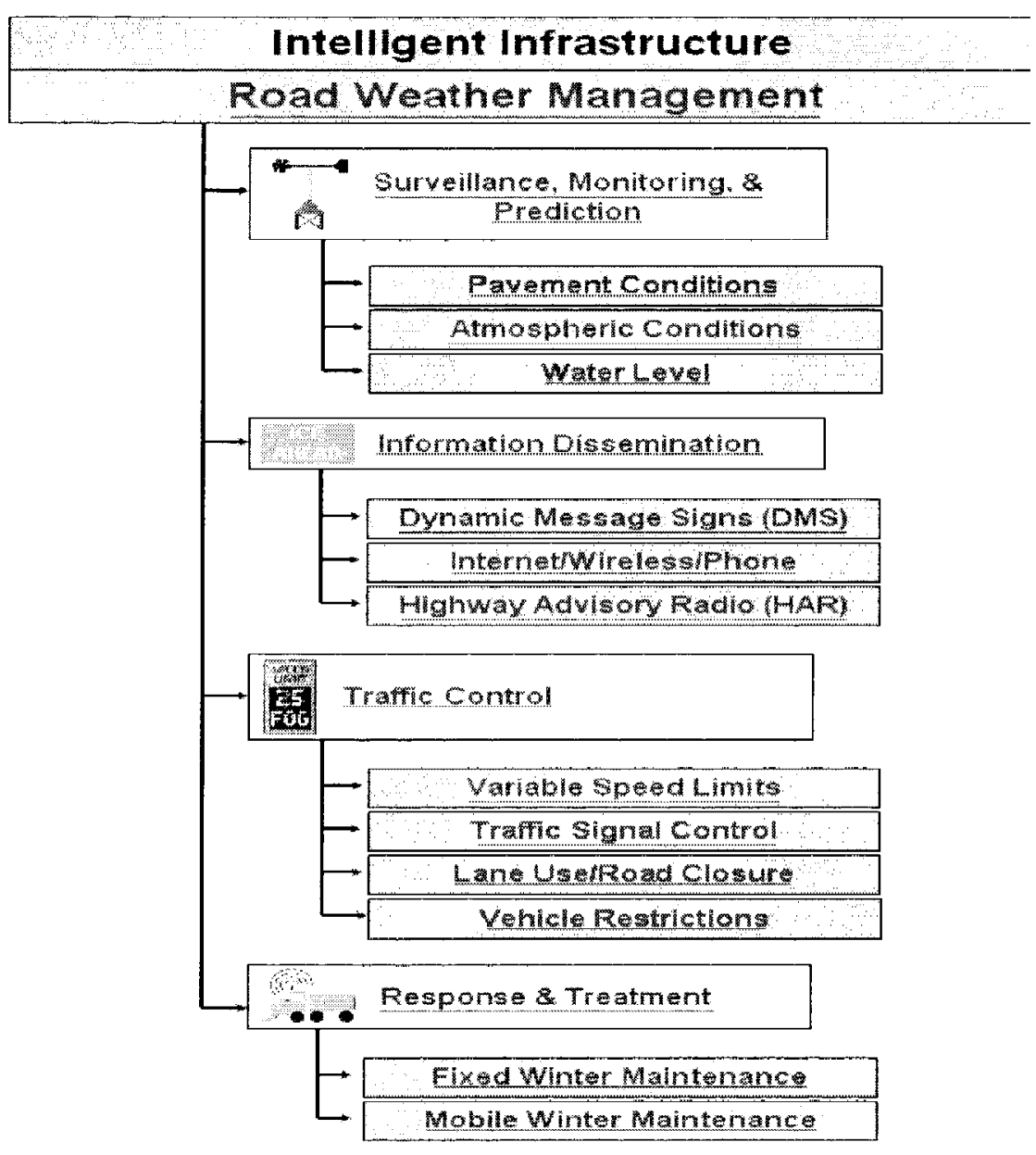

[Source: RITA, 2008]

Figure 2.17

\section{Roadway Weather Management}

\subsubsection{Commercial Vehicle Operations}

ITS applications for commercial vehicle operations are designed to improve communication between motor carriers and regulatory agencies. These include, as can be seen in Figure 2.18, electronic registration and permitting programs, electronic exchange of inspection data between regulating agencies for better inspection targeting, electronic 
screening systems, and several applications to help operators with fleet operations and security (RITA, 2008).

\subsubsection{Credentials Administration}

Electronic registration and permitting at state agencies allows carriers to register online, decreasing the turn-around time associated with permit approval. Electronic screening and credential systems deployment has been proved of having a high benefitto-cost ratio (ITS America, 2008; RITA, 2008).

\subsubsection{Safety Assurance}

Safety information Exchange (SEI) programs facilitate exchange of vehicle and driver safety information between states and jurisdictions. Enforcement personnel at check stations can use national database clearinghouses to confirm carrier regulatory compliance data and crosscheck safety assurance information (RITA, 2008).

\subsubsection{Electronic Screening}

Electronic screening applications enhance safety and efficiency for commercial vehicle operators. Carriers that provide their fleets with low-cost in-vehicle transponders can communicate with check stations and automatically transmit regulatory data to authorities as trucks approach check stations. These and other technologies such as weight-in-motion (WIM) scales improve efficiency and reduce congestion at check stations by allowing safe and legal carriers to avoid inspections and come back to the mainline without stopping (RITA, 2008; ITS America, 2008).

\subsubsection{Carrier Operations \& Fleet Management}

Several ITS technologies facilitate motor carriers with their day-to-day operations: automated vehicle location (AVL)/computer-aided design (CAD) technologies help in 
scheduling and tracking of vehicle loads; on-board monitoring of cargo can warn drivers and carriers of potentially risky load conditions; and traveler information can assist carriers choose alternate routes and departure times, evade traffic, avoid inclement weather, and arrive on time (RITA, 2008).

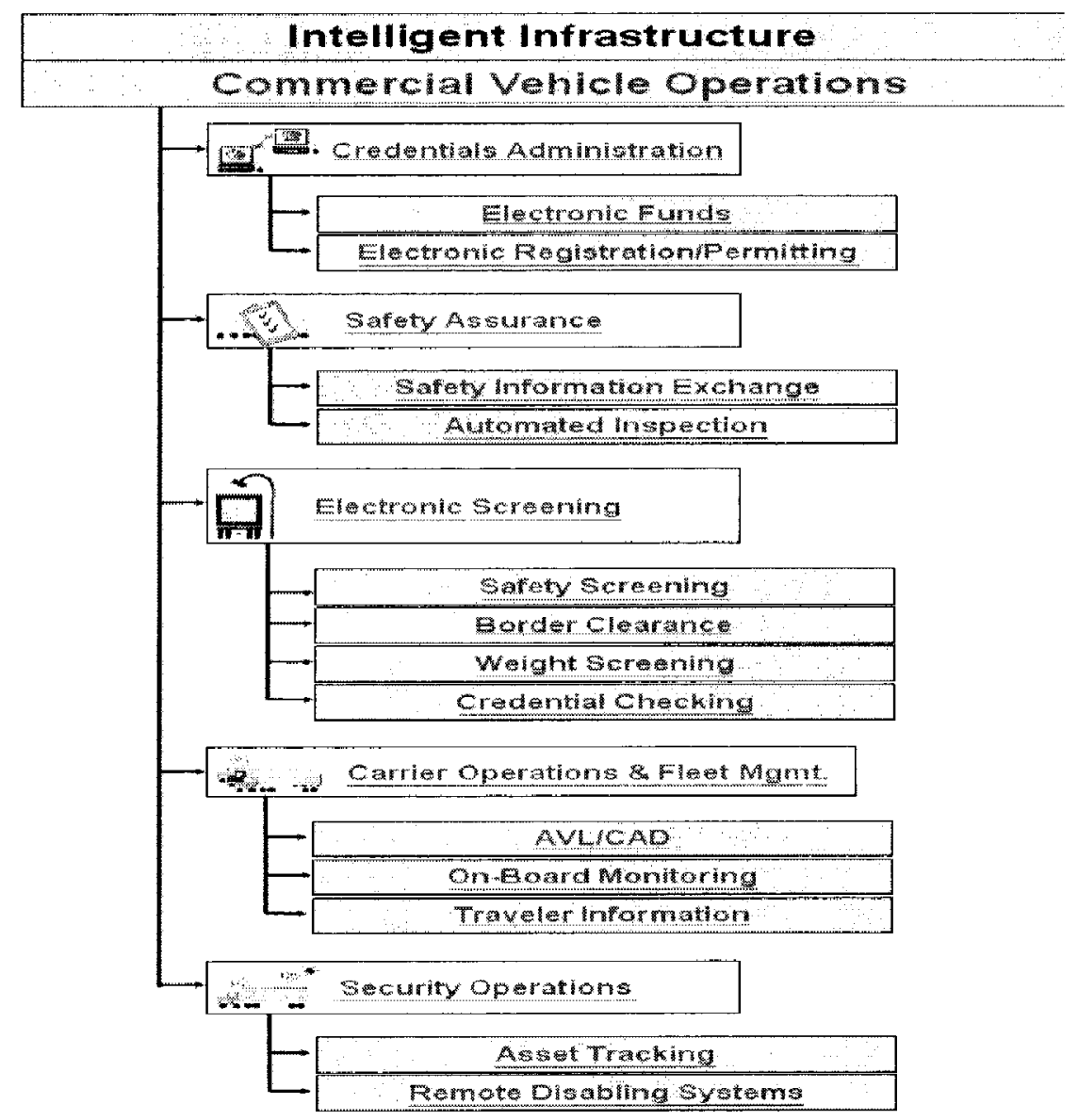

[Source: RITA, 2008]

Figure 2.18

\section{Commercial Vehicle Operations}

\subsubsection{Security Operations}

ITS applications can be used to ensure the security and safety of motor carriers.

Asset tracking technologies can supervise the location and condition of fleet assets (e.g., 
trailers, cabs, and trucks), and remote disabling systems can stop the unauthorized use of fleet vehicles and help in asset recovery (ITS America, 2008; RITA 2008).

\subsubsection{Intermodal Freight}

ITS can facilitate the protected, professional, and seamless movement of freight. Applications, as shown in Figure 2.19, being deployed provide for tracking of freight and carrier assets: containers and chassis, and improve the efficiency of freight terminal processes, drayage operations, and international border crossings (RITA,2008).

\subsubsection{Freight Tracking}

Freight tracking applications can monitor, detect, and communicate freight status information such as condition and location of goods. Also these applications ensure containerized cargo remains sealed within shipping containers while en-route (RITA, 2008; ITS America, 2008).

\subsubsection{Asset Tracking}

Many of the services possible through arterial management systems are enabled by traffic surveillance and detection technologies, such as sensors or cameras, monitoring traffic flow. The surveillance and detection technologies used to monitor traffic flow in support of ITS applications can also be used to watch main transportation facilities for security purposes (RITA, 2008).

\subsubsection{Freight Terminal Processes}

ITS freight terminal processes improve the efficiency of freight transfers or freight storage by enabling transponder tags to track cargo containers within the terminal as they are processed and sealed for transfer or storage. Electronic supply chain manifest systems, reduce the amount of time and paperwork required to transfer load and can 
improve operational efficiencies for shippers/receivers, trucking companies, and air cargo carriers (RITA, 2008; ITS America, 2008).

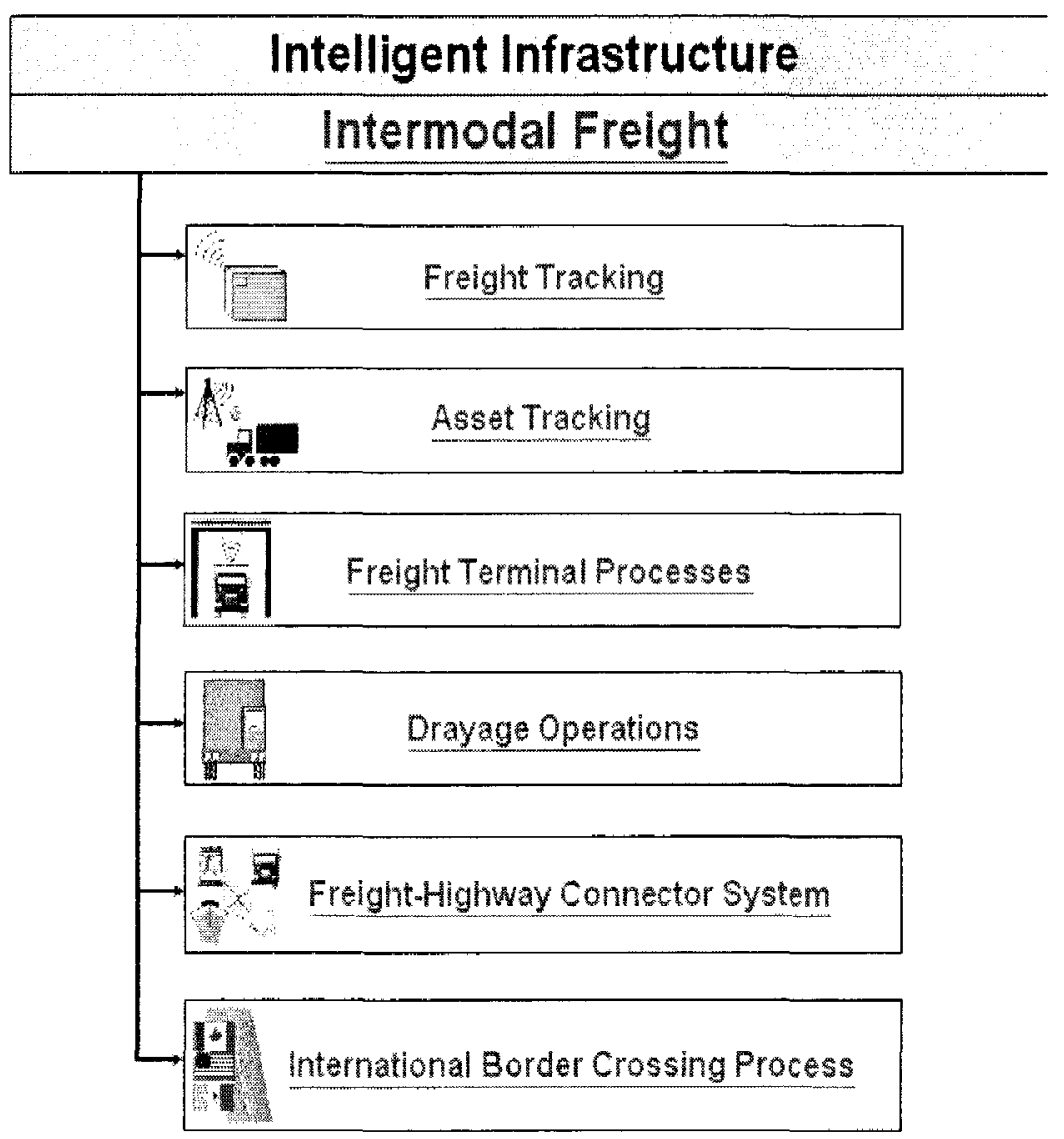

[Source: RITA, 2008]

Figure 2.19

\section{Intermodal Freight}

\subsubsection{Drayage Operations}

ITS for drayage operations facilitate the efficient loading, unloading, sorting, and transfer of cargo by implementing automated systems and robotics to optimize limited 
dock and port space. Automated truck-way technologies (automatic truck steering, speed, and platoon spacing control) can save travel time and reduce fuel consumption (RITA, 2008).

\subsubsection{Freight Highway Connector System}

ITS applications optimize traffic control and coordinate transfers near intermodal ports of entry. These applications can simplify increased freight movement on the nation's freight highway connector system (RITA, 2008).

\subsubsection{International Border Crossing Processes}

At international border crossings, automating tax revenue transactions and faster, more efficient verification of cargo manifest information can reduce delays associated with multi-agency processes. Improvements in speed, accuracy and visibility of information transfer in a freight exchange could reap large rewards for America's economic vitality. The Electronic Freight Management Initiative can assist in making these improvements through the development of a common electronic freight management (RITA, 2008; ITS America, 2008).

\subsubsection{Collision Avoidance Systems}

To enhance the capability of drivers to avoid accidents, vehicle-mounted collision warning systems (CWS) continue to be tested and deployed. These applications use a variety of sensors to monitor the vehicle's surroundings and alert the driver of conditions that could lead to a collision. Examples include forward collision warning, obstacle detection systems, and road departure warning systems as can be seen in Figure 2.20 (RITA, 2008). 


\subsubsection{Intersection Collision Warning}

Intersection collision warning systems are designed to detect and caution drivers of approaching traffic at high-speed intersections. Through the Cooperative Intersection Collision Avoidance Systems initiative, the USDOT is working in partnership with the automotive manufacturers and State and local departments of transportation to pursue an optimized combination of autonomous-vehicle, autonomous-infrastructure and cooperative communication systems that potentially address the full set of intersection crash problems (USDOT, 2008).

\subsubsection{Obstacle Detection}

Obstacle detection systems employ vehicle-mounted sensors to notice obstructions, such as other vehicles, road debris, or animals, in a vehicle's path and warn the driver. A guidance-vehicles system designed to lead traffic through heavy fog on freeways can have a high benefit-to-cost ratio (RITA, 2008; ITS Australia, 2008).

\subsubsection{Lane Change Assistance}

Lane-change warning systems have been used to alert bus and truck drivers of vehicle, or obstructions, in adjacent lanes when the driver prepares to change lanes. A National Highway Traffic Safety Administration (NHTSA) modeling study indicated that lane change/merge crash avoidance systems would be 37 percent effective (RITA, 2008). 


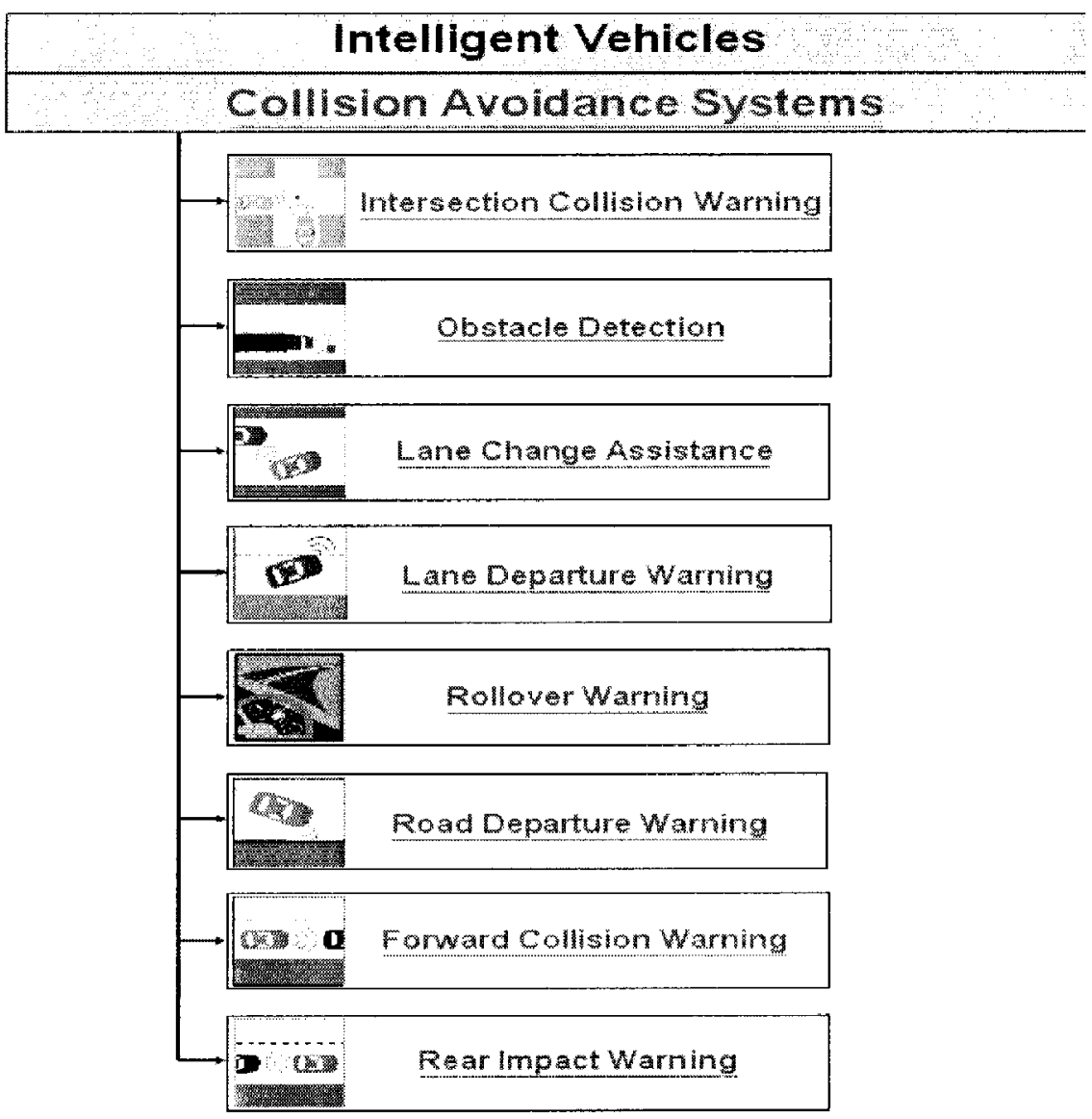

[Source: RITA, 2008]

Figure 2.20

\section{Collision Avoidance Systems}

\subsubsection{Lane Departure Warning}

Lane departure warning systems alert drivers that their vehicle is unintentionally drifting out of the lane. In-vehicle computer visioning technology designed to detect and warn truck drivers of lane departure and driver drowsiness reduced fuel consumption, increased safety, and provided drivers with more comfortable working conditions (RITA, 2008). 


\subsubsection{Rollover Warning}

Rollover warning systems warn drivers when they are traveling too fast for an approaching curve, given their vehicles operating characteristics. This has been largely a concern of heavy trucks (RITA, 2008; ITS Australia, 2008).

\subsubsection{Road Departure Warning}

Road departure warning systems have been tested using machine vision and other in-vehicle systems to sense and warn drivers of potentially risky lane-keeping practices and to keep drowsy drivers from running off the road. An NHTSA modeling study indicated that the road-departure countermeasure systems would be 24 percent effective (RITA, 2008; ITS Australia, 2008).

\subsubsection{Forward Collision Warning}

In the application area of forward-collision warning systems, microwave radar and machine vision technology facilitate perceive and avoid vehicle collisions. These systems typically use in-vehicle displays or audible alerts to notify drivers of dangerous following distances. If a driver does not properly apply brakes in a critical situation, some systems automatically assume control and apply the brakes in an attempt to avert a collision. An NHTSA modeling study indicated that forward collision warning systems would be 51 percent effective (RITA, 2008; ITS Australia, 2008).

\subsubsection{Rear Impact Warning}

Rear-impact warning systems employ radar detection to avoid accidents. A warning sign is activated on the rear of the vehicle to alert tailgating drivers of impending danger (RITA, 2008; ITS Australia, 2008). 


\subsubsection{Driver Assistance Systems}

As can be seen in Figure 2.21, numerous intelligent vehicle technologies exist to help the driver in operating the vehicle safely. Systems are available to assist with navigation, while others, such as vision enhancement and speed control systems, are intended to help safe driving during adverse conditions. Other systems assist with difficult driving tasks such as transit and commercial vehicle docking (RITA, 2008; ITS Australia, 2008).

\subsubsection{Navigation/Route Guidance}

In-vehicle navigation systems with GPS technology may lessen driver mistake, increase safety, and save time by improving driver decision in alien areas. Availability of navigational information may help reduce travel stress for drivers in unfamiliar areas (RITA, 2008; ITS Australia, 2008). 


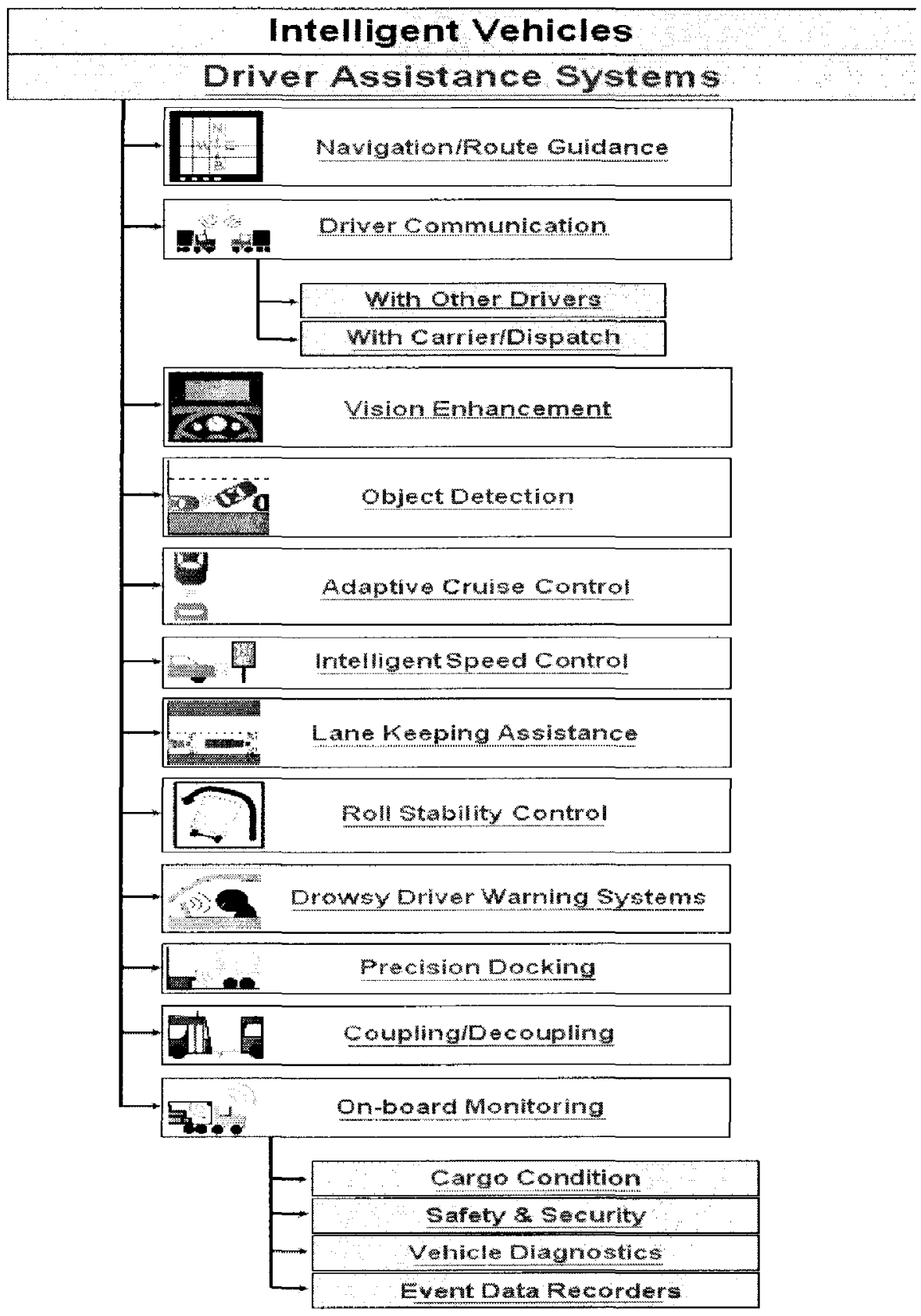

[Source: RITA, 2008]

Figure 2.21

Driver Assistance Systems 


\subsubsection{Driver Communication}

Integrated driver communication systems enable drivers and dispatchers to coordinate re-routing decisions on-the-fly and can also save time, money and enhance productivity. HAZMAT safety and securities technologies can reduce the potential for terrorists' consequences/attacks (RITA, 2008; ITS Australia, 2008).

\subsubsection{Vision Enhancement}

In-vehicle vision enhancement improves visibility for adverse driving conditions. These conditions may involve reduced sight distance due to night driving, inadequate lighting, fog, drifting snow, or other inclement weather conditions (RITA, 2008; ITS Australia, 2008).

\subsubsection{Object Detection}

Object detection system notifies the driver of an object (front, side or back) that is in the path or adjacent to the path of the vehicle, and the most common application is parking aids for passenger vehicles. An integrated countermeasure system could prevent over 48 percent of rear-end, run-off-road, and lane change crashes. Through the Integrated Vehicle-Based Safety Systems initiative, the U.S. DOT is seeking to establish a partnership with the automotive and commercial vehicle industries to accelerate the introduction of integrated vehicle-based safety systems into the Nation's vehicle fleet (RITA, 2008).

\subsubsection{Adaptive Cruise Control}

Adaptive cruise control systems maintain a driver set speed without a lead vehicle, or a specified following time if there is a lead vehicle and it is traveling slower than the set speed. An automated speed system designed to optimize travel speeds between two 
green lights can reduce fuel consumption significantly (RITA, 2008; ITS Australia, 2008).

\subsubsection{Intelligent Speed Control}

Intelligent speed control systems limit maximum vehicle speed via a signal from the infrastructure to an equipped vehicle. Intelligent Speed Adaptation (ISA) monitors the difference in speed between a vehicles current speed and its suitable speed. Corrective action can then be taken, for example, if the vehicle is traveling too fast for the current speed limit. Road beacons are used to transmit a signal to the car and warn the driver. The systems can also be automatic (RITA, 2008; ITS Australia, 2008).

\subsubsection{Lane Keeping Assistance}

Lane keeping assistance systems make small steering corrections. These systems do so when the vehicle detects an imminent lane departure without the use of a turn signal (RITA, 2008).

\subsubsection{Roll Stability Control}

Roll stability control systems apply corrective action, such as throttle control or braking. This is effected when sensors detect that a vehicle is in a possible rollover situation (RITA, 2008; ITS Australia, 2008).

\subsubsection{Drowsy Driver Warning}

Drowsy driver warning notifies the driver that he or she is fatigued which may lead to lane departure or road departure. In-vehicle computer visioning technology designed to detect and warn truck drivers of lane departure and driver drowsiness can reduce fuel consumption, increase safety, and provide drivers with more comfortable working conditions (RITA, 2008; ITS Australia, 2008). 


\subsubsection{Precision Docking}

Precision docking systems mechanize precise positioning of vehicles at loading/unloading areas. This reduces driver's stress to an extent (RITA, 2008; ITS Australia, 2008).

\subsubsection{Coupling/Decoupling}

Intelligent cruise control, speed control, guidance/steering, and coupling/decoupling systems which help transit operators link multiple buses or train cars into trains each help drivers with routine errands that weight on driver workload. These systems reduce fuel consumption also resulting in less atmospheric pollution (RITA, 2008; ITS Australia, 2008)..

\subsubsection{On-board Monitoring}

On-board monitoring applications track and report cargo condition, safety and security, and the mechanical condition of vehicles equipped with in-vehicle diagnostics. This information is presented to the driver immediately, transmitted off-board, or stored. In the event of a crash or near-crash, in-vehicle event data recorders record vehicle performance data and other input from video cameras or radar sensors to improve the post-accident processing of data (RITA, 2008; ITS Australia, 2008).

\subsubsection{Collision Notification Systems}

In an effort to improve response times and save lives, collision notification systems have been designed to detect and report the location and severity of incidents to agencies and services responsible for coordinating appropriate emergency response actions. These systems can be activated manually (Mayday), or automatically with automatic collision 
notification $(\mathrm{ACN})$, and advanced systems may transmit information on the type of crash, number of passengers, and the likelihood of injuries (RITA, 2008; ITS Australia, 2008).

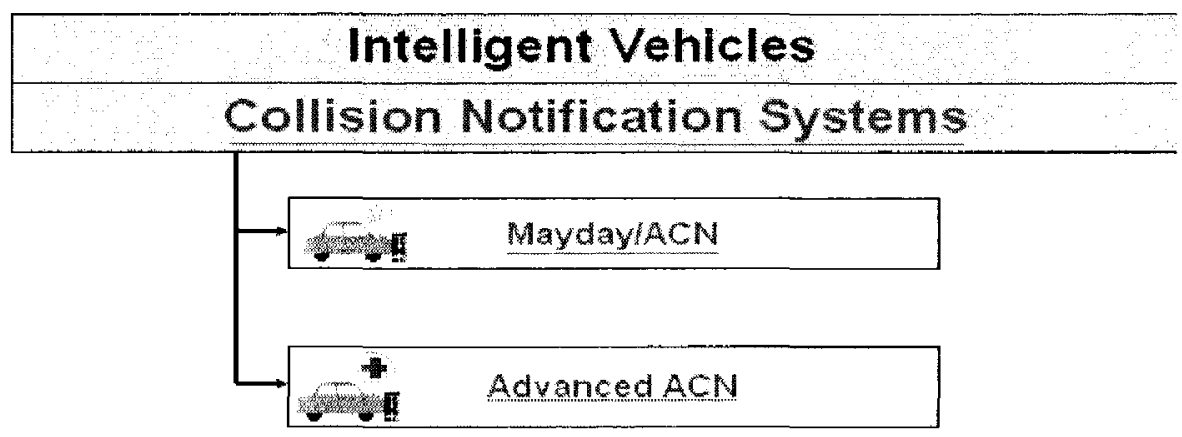

[Source: RITA, 2008]

Figure 2.22

\section{Collision Notification Systems}

\subsubsection{Mayday/ACN}

The typical Mayday/ACN product uses location technology, wireless communication, and a third-party response center to alert the closest Public Safety Answering Point (PSAP) for emergency response. Drivers equipped with in-vehicle emergency communications found feel "more secure" with Mayday voice communications (RITA, 2008; ITS Australia, 2008).

\subsubsection{Advanced ACN}

Advanced collision notification systems utilize in-vehicle crash sensors, GPS technology, and wireless communications systems to provide public/private call centers with crash location information. Also sometimes, these systems inform about the number of injured passengers and the nature of their injuries (RITA, 2008; ITS Australia, 2008). 


\subsubsection{Other Applications}

\subsubsection{Smart Glasses}

Glasses that monitor eye movement and alert drivers when they become fatigued will soon be available to motorists in an initiative to curb the number of fatalities in Australia. The glasses have frames fitted with tiny sensors that measure the size and speed of blinking and beep when motorist is too tired to drive safely (ITS, Australia, 2008).

\subsubsection{Real-time Rail Time Table on iPhone}

Listed as "Live UK rail journey planner" under travel on the iPhone web applications, KIZOOM.MOBI outclasses other time-table only services by delivering free and easy-to-navigate real-time rail time tables, fast journey planning, taxi finder, disruption news and route stops. It is a really useful bundle of travel services for people on the move (ITS UK, 2008).

\subsubsection{Smart Roads}

There has been a great discussion in US about the smart roads of tomorrow for years. But now "intelligent highway" technology is inching into the mainstream. One reason is that the number of vehicles on the road is increasing far faster than the ability to add more roads or widen old ones. Consider these statistics from the Federal Highway Administration (FHWA). Over the past 20 years, about $2 \%$ more streets and roads have been built, but there are more than $50 \%$ more vehicles using them-and they travel $77 \%$ more miles.

The result is spreading congestion that is affecting broader metropolitan areas. Pending an unlikely swing away from personal vehicles, traffic planners figure the best 
solution is to manage traffic flow more efficiently. That's where smart highways come in. A number of possible solutions were demonstrated during a big automated highway expo in San Diego. Among them is high-speed "platooning" in which a smart highway takes control of cars and moves them along, bumper-to-bumper, at 70 miles an hour. Those technologies are years away from implementation. More immediate traffic aids include "smart" traffic signals and roadside displays that give drivers instant updates on traffic conditions ahead. The potential U.S. market for smart road technology is enormous. A study released two years ago by the U.S. Department of Transportation pegs it at some \$430 billion over the 20-year period from 1996 to 2016 (FHWA, 2008).

\subsection{Artificial Neural Networks (ANN)}

An Artificial Neural Network (ANN) or commonly just Neural Network (NN) is an interconnected group of artificial neurons that uses a mathematical model or computational model for information processing based on a connectionist approach to computation. In most cases an $\mathrm{ANN}$ is an adaptive system that changes its structure based on external or internal information that flows through the network. In more practical terms neural networks are non-linear statistical data modeling tools. They can be used to model complex relationships between inputs and outputs or to find patterns in data (Flood and Kartam, 1998).

Neural networks, with their remarkable ability to derive meaning from complicated or imprecise data, can be used to extract patterns and detect trends that are too complex to be noticed by either humans or other computer techniques. A trained neural network can be thought of as an "expert" in the category of information it has been given to analyze. This expert can then be used to provide projections given new situations of interest and 
answer "what if" questions.

Other advantages include:

1. Adaptive learning: An ability to learn how to do tasks based on the data given for training or initial experience.

2. Self-Organization: An ANN can create its own organization or representation of the information it receives during learning time.

Neural networks take a different approach to problem solving than that of conventional computers. Conventional computers use an algorithmic approach i.e. the computer follows a set of instructions in order to solve a problem. Unless the specific steps that the computer needs to follow are known the computer cannot solve the problem. That restricts the problem solving capability of conventional computers to problems that we already understand and know how to solve. But computers would be so much more useful if they could do things that we don't exactly know how to do (Flood and Kartam, 1998).

Neural networks process information in a similar way the human brain does. The network is composed of a large number of highly interconnected processing elements (neurons) working in parallel to solve a specific problem. Neural networks learn by example. They cannot be programmed to perform a specific task. The examples must be selected carefully otherwise useful time is wasted or even worse the network might be functioning incorrectly. The disadvantage is that because the network finds out how to solve the problem by itself, its operation can be unpredictable (Flood and Kartam, 1998).

In summary one can say that: 
- Artificial neural networks (ANN) are AI application which has recently been used widely to model some of the human interesting activities in many areas of science and engineering

- Engineers often deal with incomplete and noisy data which is one area where ANNs are most applicable

- The special characteristics of ANNs make them a promising candidate for modeling some of the difficult engineering problems

- One of the distinct characteristics of the ANN is its ability to learn and generalize from experience and examples and adapt to the changing situations. In simple terms, an ANN tries to imitate some of the learning activities of the human brain.

\subsubsection{Biological Neuron and Artificial Neuron}

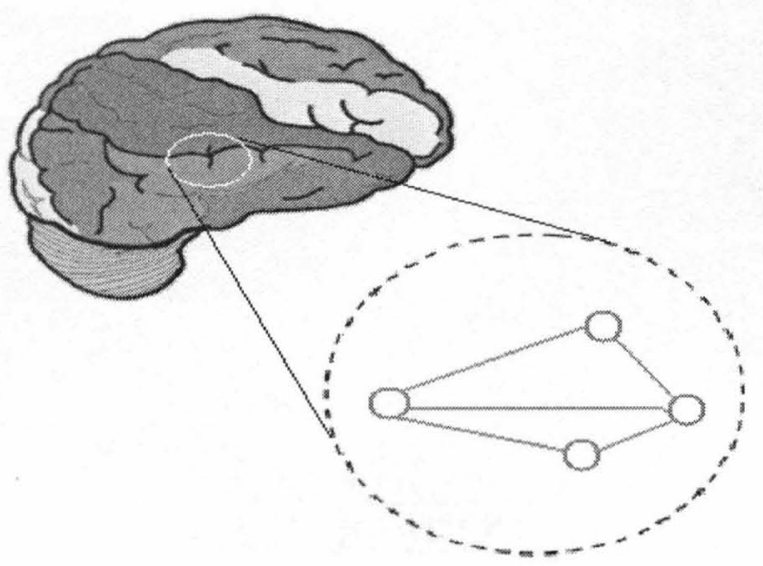

- ANNs are much simpler than the human brain

- The human brain consists 100 billion neurons and 100 trillion connections (synapses) between them.

- Many highly specialized types of neurons (which are not possible in ANN) exist in human brain (Fraser, 2008). 


\subsubsection{Biological Neuron}

The basic model of the neuron is founded upon the functionality of a biological neuron. "Neurons are the basic signaling units of the nervous system" and "each neuron is a discrete cell whose several processes arise from its cell body".

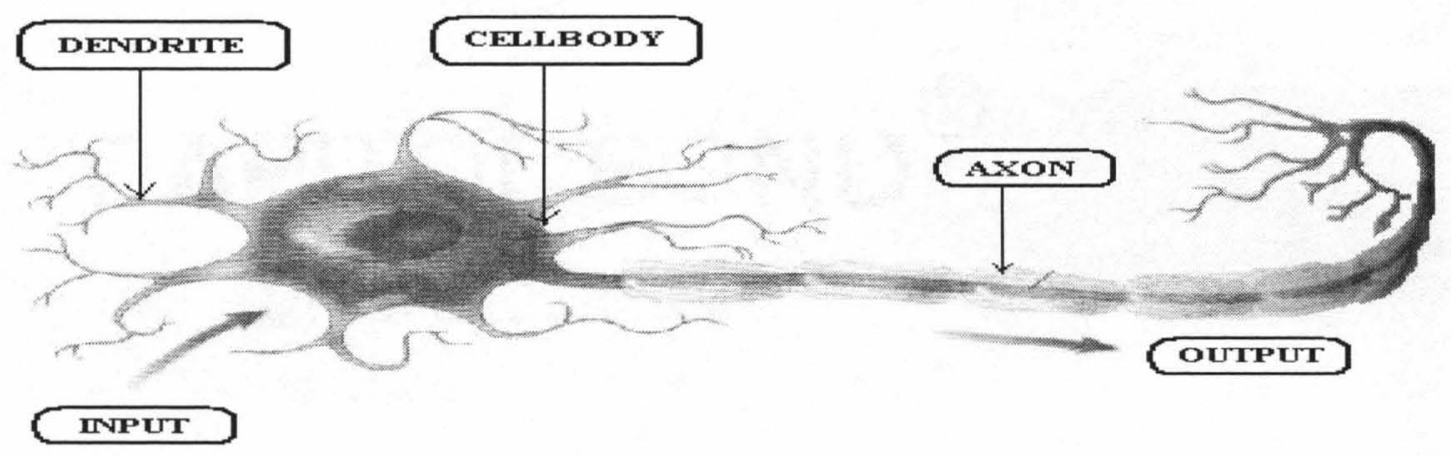

[Source: V. Giri, 2006]

Figure 2.23

\section{Biological Neuron}

The neuron has four main regions to its structure. The cell body, or soma, has two offshoots from it, the dendrites, and the axon, which end in pre-synaptic terminals. The cell body is the heart of the cell, containing the nucleus and maintaining protein synthesis. A neuron may have many dendrites, which branch out in a treelike structure, and receive signals from other neurons. A neuron usually only has one axon which grows out from a part of the cell body called the axon hillock. The axon conducts electric signals generated at the axon hillock down its length. These electric signals are called action potentials. The other end of the axon may split into several branches, which end in a pre-synaptic terminal. Action potentials are the electric signals that neurons use to convey information to the brain. All these signals are identical. Therefore, the brain 
determines what type of information is being received based on the path that the signal took. The brain analyzes the patterns of signals being sent and from that information it can interpret the type of information being received. Myelin is the fatty tissue that surrounds and insulates the axon. Often short axons do not need this insulation. There are uninsulated parts of the axon. These areas are called Nodes of Ranvier. At these nodes, the signal traveling down the axon is regenerated. This ensures that the signal traveling down the axon travels fast and remains constant (i.e. very short propagation delay and no weakening of the signal). The synapse is the area of contact between two ncurons. The neurons do not actually physically touch. They are separated by the synaptic cleft, and electric signals are sent through chemical interaction. The neuron sending the signal is called the presynaptic cell and the neuron receiving the signal is called the postsynaptic cell. The signals are generated by the membrane potential, which is based on the differences in concentration of sodium and potassium ions inside and outside the cell membrane. Ncurons can be classified by their number of processes (or appendages), or by their function. If they are classified by the number of processes, they fall into three categories. Unipolar neurons have a single process (dendrites and axon are located on the same stem), and are most common in invertebrates. In bipolar neurons, the dendrite and axon are the neuron's two separate processes. Bipolar neurons have a subclass called pseudo-bipolar neurons, which are used to send sensory information to the spinal cord. Finally, multipolar neurons are most common in mammals. Examples of these neurons are spinal motor neurons, pyramidal cells and Purkinje cells (in the cerebellum). If classified by function, neurons again fall into three separate categories. The first group is sensory, or afferent, neurons, which provide information for perception and motor 
coordination. The second group provides information (or instructions) to muscles and glands and is therefore called motor neurons. The last group, interneuronal, contains all other neurons and has two subclasses. One group called relay or projection interneurons have long axons and connect different parts of the brain. The other group called local interneurons are only used in local circuits (Kandel et al., 2000).

\subsubsection{Artificial Neuron}

When creating a functional model of the biological neuron, there are three basic components of importance. First, the synapses of the neuron are modeled as weights. The strength of the connection between an input and a neuron is noted by the value of the weight. Negative weight values reflect inhibitory connections, while positive values designate excitatory connections. The next two components model the actual activity within the neuron cell. An adder sums up all the inputs modified by their respective weights. This activity is referred to as linear combination. Finally, an activation function controls the amplitude of the output of the neuron. An acceptable range of output is usually between 0 and 1 , or -1 and 1 (Demuth et al., 2006).

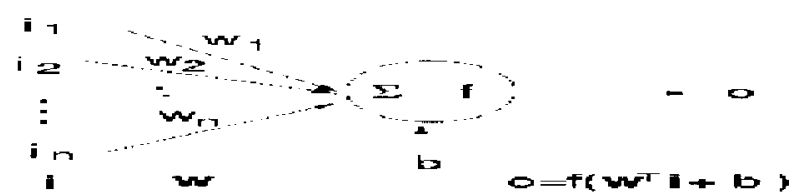

[Source: Neural Network Toolbox User's Guide, MathWorks, Inc., 2006]

Figure 2.24

\section{Artificial Neuron}


Like the brain, a neural network is a massively parallel collection of small and simple processing units where the interconnections form a large part of the network's intelligence.

\subsubsection{ANN Features}

- Large number of very simple units.

- Connections through weighted links.

- There is no "program". The "program" is the architecture of the network.

- There is no central control. If a portion of the network is damaged, the network is still functional.

- A human observer can't understand what is going on inside the network. It is a sort of a "black box" (Lingireddy and Ormsbee, 1998).

\subsubsection{Neural Network Topology (Feed Forward Neural Networks)}

The feedforward neural network was the first and arguably simplest type of artificial neural network devised. In this network, the information moves in only one direction, forward, from the input nodes, through the hidden nodes (if any) and to the output nodes. There are no cycles or loops in the network (Lingireddy and Ormsbee, 1998). 


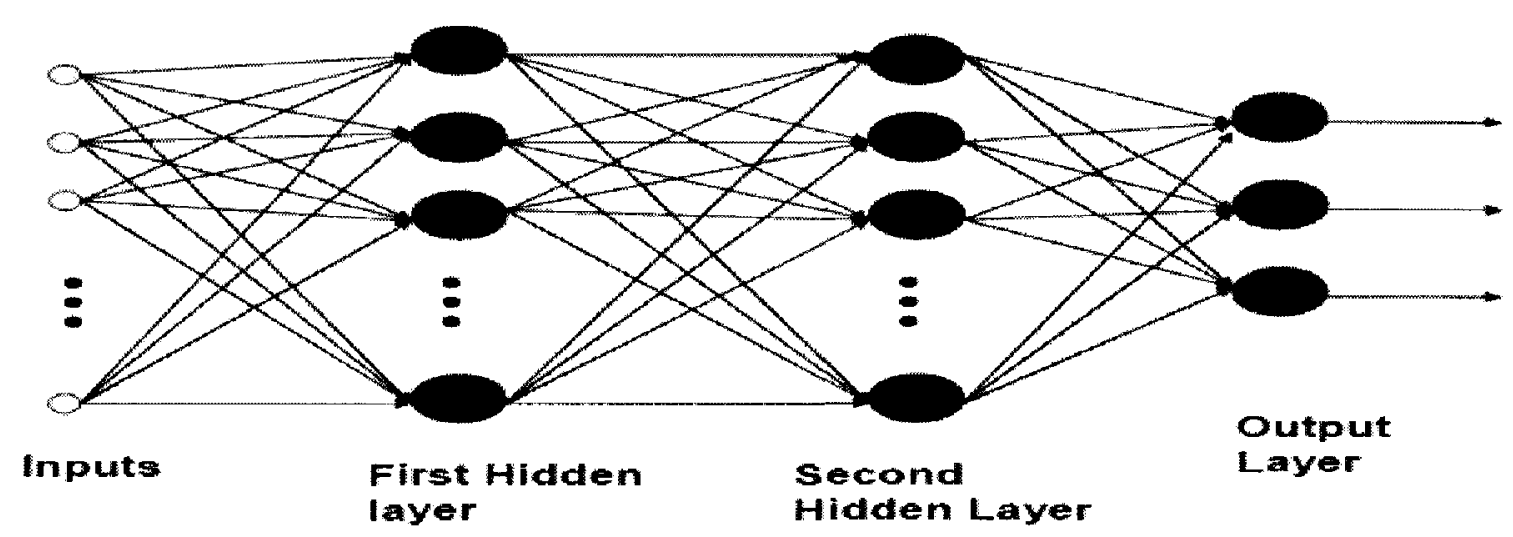

[Source: Neural Network Toolbox User's Guide, MathWorks, Inc., 2006]

Figure 2.25

\section{Feed Forward Neural Networks}

\subsubsection{Activation Functions}

The main difference between network types lies in the type of activation function used by the hidden neuron. The activation function acts as a squashing function, such that the output of a neuron in a neural network is between certain values (usually 0 and 1 , or 1 and 1). In general, there are four types of activation functions:

- Sign Function (Classification, Pattern Recognition)

- Step Function (Classification, Pattern Recognition)

- Sigmoid Function (Squashes inputs so outputs are within 0-1)

- Linear Function (Used for linear approximation) 

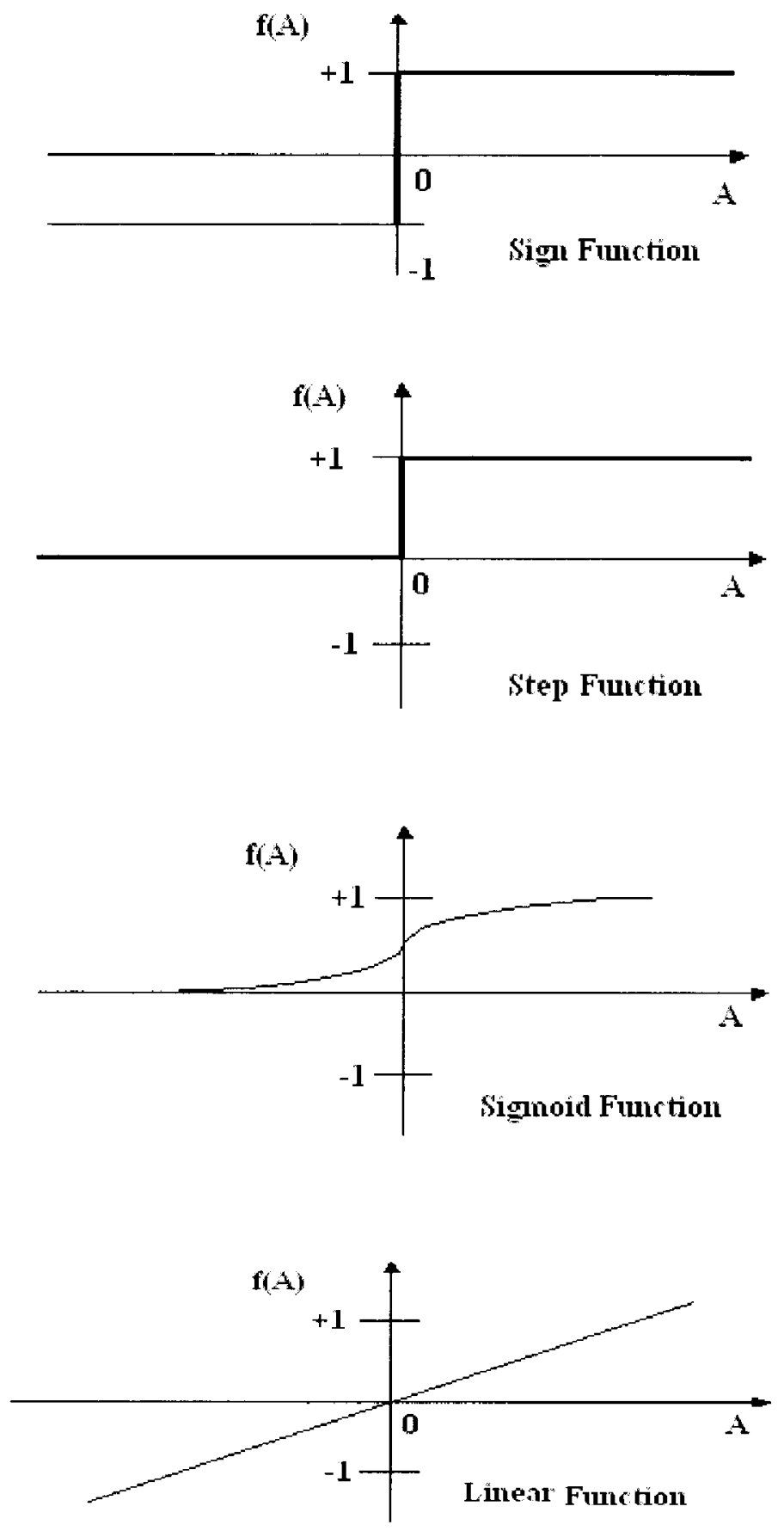

[Source: Neural Network Toolbox User's Guide, MathWorks, Inc., 2006]

Figure 2.26

Types of Activation Function 


\subsubsection{Training an ANN}

Once a network has been structured for a particular application, that network is ready to be trained. To start this process the initial weights are chosen randomly. Then, the training, or learning, begins. There are two approaches to training - supervised and unsupervised. Supervised training involves a mechanism of providing the network with the desired output either by manually "grading" the network's performance or by providing the desired outputs with the inputs. Unsupervised training is where the network has to make sense of the inputs without outside help. The vast bulk of networks utilize supervised training. Unsupervised training is used to perform some initial characterization on inputs (Kandel et al., 2000).

\subsubsection{Supervised Training}

In supervised training, both the inputs and the outputs are provided. The network then processes the inputs and compares its resulting outputs against the desired outputs. Errors are then propagated back through the system, causing the system to adjust the weights which control the network. This process occurs over and over as the weights are continually tweaked. The set of data which enables the training is called the "training set." During the training of a network the same set of data is processed many times as the connection weights are ever refined (Kandel et al., 2000).

\subsubsection{Unsupervised Training}

The other type of training is called unsupervised training. In unsupervised training, the network is provided with inputs but not with desired outputs. The system itself must then decide what features it will use to group the input data. This is often referred to as self-organization or adaption (Kandel et al., 2000). 


\subsubsection{Various issues with ANN}

- Selecting topology

- Preprocessing training data

- Data selection for training

- Duration of ANN training

- Speeding up the training process

- Checking network performance

\subsection{Kendall's Tau Test and Multiple-Regression (MR)}

\subsubsection{Kendall's Tau Test}

The Kendall tau rank correlation coefficient (or simply the Kendall tau coefficient, Kendall's $\tau$ or Tau test(s)) is used to measure the degree of correspondence between two rankings and assessing the significance of this correspondence. It best depicts the degree of association for non-parametric data (Wilkie, 1980). In other words, it measures the strength of association of the cross tabulations. It was developed by Maurice Kendall in 1938.

The Kendall tau coefficient $(\tau)$ has the following properties:

- If the agreement between the two rankings is perfect (i.e., the two rankings are the same) the coefficient has value 1.

- If the disagreement between the two rankings is perfect (i.e., one ranking is the reverse of the other) the coefficient has value -1 . 
- For all other arrangements the value lies between -1 and 1 , and increasing values imply increasing agreement between the rankings. If the rankings are completely independent, the coefficient has value 0 on average.

Kendall tau coefficient is defined

$$
\tau=\frac{2 P}{\frac{1}{2} n(n-1)}-1=\frac{4 P}{n(n-1)}-1
$$

where $n$ is the number of items, and $P$ is the sum, over all the items, of items ranked after the given item by both rankings (Wilkie, 1980).

\subsubsection{Multiple-Regression (MR)}

In statistics, Multiple linear regression is a regression method that allows the relationship between the dependent variable $Y$ and the $p$ independent variables $\mathrm{X}$ and a random term $\varepsilon$. The model can be written as

$$
Y=\beta_{1}+\beta_{2} X_{2}+\cdots+\beta_{p} X_{p}+\varepsilon
$$

where $\beta_{1}$ is the intercept ("constant" term), the $\beta_{i}$ s are the respective parameters of independent variables, and $p$ is the number of parameters to be estimated in the linear regression.

Non-linear model can be converted into linear ones but one has to be very careful while converting as some models can not be made linear (Haan, 1977). 


\section{CHAPTER III}

\section{DATA COLLECTION}

Data collection is a term used to describe the process of preparing and collecting the various types of information related to a research project. The majority of data collection usually takes place early on in a project, and is often formalized through a data collection plan which often contains the following activities.

Pre-collection activity - defined goals, target data types and sources, data definitions, collection methods, collection locations including internet, personal interviews, public input, field surveys (historical data and project specific).

Collection - data collection including list of sources searched or contacted

Present findings - usually involves some form of data compilation or reduction, organization and sorting analysis and/or presentation.

A formal data collection process is necessary as it ensures data gathered are both defined and accurate and subsequent decisions based on arguments embodied in the findings have objective validity (Sapsford and Jupp, 1996).

Since this research involved identifying, describing, quantifying, and modeling the relationship between Intelligent Transportation Systems (ITS) applications and ambient air pollutant concentrations, target data were mainly related to ambient air pollution records, ITS application types, and transportation system characteristics in urbanized areas. 
Data pertaining to fifty-nine USA cities (those specified as federal-aid urbanized areas) were used in this research. Most data components were obtained through authenticated web-pages of various municipal, state, and federal government organizations as described in the following sections.

\subsection{Air Data}

The AirData web site of Environmental Protection Agency (EPA) provides access to historical and current air pollution measurement records for the entire United States. AirData produces summary reports and maps of air pollution values based on criteria that one may specify in terms of date, location, and data type. AirData makes available annual summaries of air pollution data from two EPA databases:

- AQS (Air Quality System) database provides air monitoring records specific ambient concentration measurements of criteria-defined and hazardous air pollutants at monitoring sites, primarily in cities and towns.

- NEI (National Emission Inventory) database provides estimates of annual emission levels of criteria and hazardous air pollutants from all types of regulated sources. The NEI database in 2002 replaced two separate EPA databases for emissions of criteria air pollutants (National Emission Trends, or NET) and hazardous air pollutants (National Toxics Inventory, or NTI).

In this research, the Monitor Data Queries section and procedures of the AirData Web site were used to access and generate specific and detailed descriptions of air monitor records, and annual summary data details for more pollutants and years than 
standard AirData reports and maps provide. Monitor Data Queries uses data extracted from the AQS database (EPA, 2008).

Arithmetic mean-measure of central tendency obtained from the sum of the observed pollutant data values over a specified time interval divided by the number of values that comprise the sum for the monitor year for a specific monitor location-was used to define the average concentration of a pollutant in ambient air over an urbanized area. Observation count of each monitor location (ID) was multiplied with the arithmetic mean (ambient air concentration of a pollutant) for the corresponding year and the average concentration was defined by summing the resultant for all monitors and dividing by the total number of observation count for all monitors. The average concentrations for three criteria air pollutants-- $\mathrm{CO}, \mathrm{NO}_{2}$, and $\mathrm{O}_{3}-$ were calculated for 59 cities of US for years $2000,2002,2004$, and 2005. The concentrations (in ppm) of these three pollutants in ambient air were termed the Target Variables in this study and were named as Y1, Y2, and $\mathrm{Y} 3$ respectively as shown in Table 3.2 and Appendix-I.

\subsection{Transportation/Highway Data}

While the Office of Highway Policy Information is responsible for the preparation of the publication of transportation/highway data, a number of the statistical summaries are prepared by other units within the Federal Highway Administration (FHWA). All highway data are submitted to the FHWA by the individual States. Each State is analyzed for consistency against its own past years of data and also against other State and Federal data. The result is the publication of annual series-highway statistics-- of selected 
statistical tabulations relating to highway/transportation in three major areas: (1) highway use-the ownership and operation of motor vehicles; (2) highway finance-the receipts and expenditures for highways by public agencies; and (3) the highway plant- the extent, characteristics, and performance of the public highways, roads, and streets across the nation.

In this research, Highway Statistics 2000 through 2005 were the resource used to extract data related to the Federal-Aid Urbanized Areas of US. A "Federal-Aid Urbanized Area" is an area with 50,000 or more persons that at a minimum encompasses the land area delineated as the urbanized area by the Bureau of the Census (Highway Statistics, 2005).

The descriptive quantities utilized in this work included population, road length, Daily Vehicle Miles Traveled (DVMT), land area, freeway DVMT, freeway miles, and annual average daily traffic on freeways. These quantities were considered the most appropriate input variables associated with transportation and ambient air quality. In this research, these were designated with variable names $\mathrm{X} 1$ through $\mathrm{X} 7$ respectively. Duc importance was given to frecway data in this research for the reason that freeway constitute only 2.8 percent of all roadway lengths in the nation, yet 37 percent of total DVMT are on freeways (Highway Statistics, 2005). Detailed data for these variables (from X1 through X7) are listed in Appendix-I.

\subsection{ITS Data}

Intelligent Transportation Systems (ITS) deployment intensity data for the fiftynine US cities were downloaded from the RITA, US DOT, web site (RITA, 2008). 
Table 3.1 below provides a sample of the Deployment Statistics. ITS applications have been grouped under eight bundles: Freeway Management, Incident Management, Arterial Management, Electronic Toll Collection, Transit Management, Electronic Fare Payment, Highway Rail Intersections, and Emergency Management. Column under the name "Reported" indicates the number of miles/ramps under a specific ITS application in a bundle and the column under the name "Total" represents the total number of miles/ramps under that bundle. Therefore, "Percent" indicates the application intensity in percent of an ITS user service in an area calculated by dividing "Reported" with "Total" and then multiplying with 100 .

Similarly, seventy-eight tables related to major metropolitan areas were available from RITA and were used to compile application intensity for each of the fifty-nine US cities described earlier. As shown in Table 3.1, there is a total of thirty ITS applications that are included in the surveys. Only seventeen of the thirty applications' data (percentdata) which are more associated with air pollution generation were used for this research and were included as variables named X8 to X24 as defined in the Table 3.2. The data for $\mathrm{X} 8$ through $\mathrm{X} 24$ (termed as input variables) are listed in Appendix-I.

Data collection process is crucial in any research because reliability, specificity, and rationale of the data collected ensure the objective validity of the outcome of that research. In this research, the data were collected as mentioned in earlier sections as per the data collection plan keeping goals and objective in the view. The next chapter "Methodology" describes how these data were acted upon to arrive at the results and findings. 
Table 3.1 National Summary (FY-2005)

(78 metropolitan areas surveyed)

\begin{tabular}{|c|c|c|c|}
\hline Freeway Management & Reported & Total & Percent \\
\hline Miles under electronic surveillance & 6503 & 17090 & $38 \%$ \\
\hline Ramps controlled by ramp meter & 5284 & 25198 & $21 \%$ \\
\hline Miles under lane control & 1478 & 17090 & $9 \%$ \\
\hline Number of Dynamic Message Signs (DMS) & 3177 & $\mathrm{~N} / \mathrm{A}^{* *}$ & $\mathrm{~N} / \mathrm{A}^{* *}$ \\
\hline Miles covered by Highway Advisory Radio(HAR) & 3722 & 17090 & $22 \%$ \\
\hline \multicolumn{4}{|l|}{ Incident Management } \\
\hline Freeway miles under incident detection algorithms & 2732 & 17090 & $16 \%$ \\
\hline $\begin{array}{l}\text { Freeway miles under free cell phone call to a dedicated } \\
\text { number }\end{array}$ & 4703 & 17090 & $28 \%$ \\
\hline $\begin{array}{l}\text { Freeway miles covered by surveillance cameras } \\
\text { (CCTV) }\end{array}$ & 5984 & 17090 & $35 \%$ \\
\hline Freeway miles covered by service patrols & 8241 & 17090 & $48 \%$ \\
\hline Arterial miles under incident detection algorithms & 1481 & 97687 & $2 \%$ \\
\hline $\begin{array}{l}\text { Arterial miles under free cell phone call to a dedicated } \\
\text { number }\end{array}$ & 4148 & 97687 & $4 \%$ \\
\hline Arterial miles covered by surveillance cameras (CCTV) & 4912 & 97687 & $5 \%$ \\
\hline Arterial miles covered by service patrols & 10338 & 97687 & $11 \%$ \\
\hline \multicolumn{4}{|l|}{ Arterial Management } \\
\hline $\begin{array}{l}\text { Signalized intersections covered by electronic } \\
\text { surveillance }\end{array}$ & 49754 & 138261 & $36 \%$ \\
\hline $\begin{array}{l}\text { Signalized intersections under centralized or closed } \\
\text { loop control }\end{array}$ & 73295 & 138261 & $53 \%$ \\
\hline Number of Dynamic Message Signs (DMS) & 1142 & N/A** & $\mathrm{N} / \mathrm{A}^{* *}$ \\
\hline $\begin{array}{l}\text { Arterial miles covered by Highway Advisory Radio } \\
\text { (HAR) }\end{array}$ & 1942 & 97687 & $2 \%$ \\
\hline \multicolumn{4}{|l|}{ Electronic Toll Collection } \\
\hline $\begin{array}{l}\text { Toll collection plazas with Electronic Toll Collection } \\
\text { capabilities }\end{array}$ & 730 & 777 & $94 \%$ \\
\hline $\begin{array}{l}\text { Toll collection lanes with Electronic Toll Collection } \\
\text { capabilities }\end{array}$ & 3862 & 4907 & $79 \%$ \\
\hline \multicolumn{4}{|l|}{ Transit Management } \\
\hline $\begin{array}{l}\text { Fixed route buses equipped with Automatic Vehicle } \\
\text { Location (AVL) }\end{array}$ & 27021 & 49612 & $54 \%$ \\
\hline $\begin{array}{l}\text { Fixed route buses with electronic real-time monitoring } \\
\text { of system components }\end{array}$ & 16108 & 49612 & $32 \%$ \\
\hline $\begin{array}{l}\text { Demand responsive vehicles that operate under } \\
\text { Computer Aided Dispatch (CAD) }\end{array}$ & 4710 & 10530 & $45 \%$ \\
\hline $\begin{array}{l}\text { Bus stops with electronic display of dynamic traveler } \\
\text { information to the public }\end{array}$ & 1670 & 536333 & $0 \%$ \\
\hline
\end{tabular}




\begin{tabular}{|l|l|l|l|}
\hline $\begin{array}{l}\text { Fixed route buses equipped with Magnetic Stripe } \\
\text { Readers }\end{array}$ & 29800 & 49612 & $60 \%$ \\
\hline Fixed route buses equipped with Smart Card Readers & 11349 & 49612 & $23 \%$ \\
\hline Rail Stations equipped with Magnetic Stripe Readers & 1174 & 2807 & $42 \%$ \\
\hline Rail Stations equipped with Smart Card Readers & 349 & 2807 & $12 \%$ \\
\hline Highway Rail Intersections & 11095 & $5 \%$ \\
\hline Highway rail intersections under electronic surveillance & 522 & 112841 & $80 \%$ \\
\hline Emergency Management & 90836 & 112841 & $18 \%$ \\
\hline Vehicles under Computer Aided Dispatch (CAD) & 20065 & & \\
\hline $\begin{array}{l}\text { Vehicles equipped with on-board navigation } \\
\text { capabilities }\end{array}$ & & & \\
\hline *NR=No Response, **N/A=Not Applicable & & & \\
\hline \multicolumn{4}{|l|}{} \\
\hline http://www.itsdeployment.its.dot.gov/
\end{tabular}

Table 3.2 Variables

\begin{tabular}{|c|l|c|}
\hline $\begin{array}{c}\text { Designation of } \\
\text { Variable }\end{array}$ & & Description \\
\hline $\mathrm{X} 1$ & Population in thousands & Input \\
\hline $\mathrm{X} 2$ & Road Length in miles & Input \\
\hline $\mathrm{X} 3$ & Daily Vehicle Miles Traveled (DVMT) in thousands & Input \\
\hline $\mathrm{X} 4$ & Land Area in square miles & Input \\
\hline $\mathrm{X} 5$ & Freeway DVMT in thousands & Input \\
\hline $\mathrm{X} 6$ & Freeway Miles & Input \\
\hline $\mathrm{X} 7$ & Annual average daily traffic on freeways & Input \\
\hline $\mathrm{X} 8$ & $\%$ of Freeway Miles covered by Highway Advisory Radio (HAR) & Input \\
\hline $\mathrm{X} 9$ & $\%$ of Freeway miles under incident detection algorithms & Input \\
\hline $\mathrm{X} 10$ & $\%$ of Freeway miles covered by surveillance cameras (CCTV) & Input \\
\hline $\mathrm{X} 11$ & $\%$ of Freeway miles covered by service patrols & Input \\
\hline
\end{tabular}




\begin{tabular}{|c|c|c|}
\hline $\mathrm{X} 12$ & $\%$ of Arterial miles under incident detection algorithms & Input \\
\hline $\mathrm{X} 13$ & $\%$ of Arterial miles covered by surveillance cameras (CCTV) & Input \\
\hline $\mathrm{X} 14$ & $\%$ of Arterial miles covered by service patrols & Input \\
\hline$\overline{\mathrm{X} 15}$ & $\%$ of Signalized intersections covered by electronic survcillance & Input \\
\hline $\mathrm{X} 16$ & $\begin{array}{l}\% \text { of Signalized intersections under centralized or closed loop } \\
\text { control }\end{array}$ & Input \\
\hline $\mathrm{X} 17$ & $\%$ of Arterial miles covered by Highway Advisory Radio (HAR) & Input \\
\hline $\mathrm{X} 18$ & $\begin{array}{l}\% \text { of Toll collection lanes with Electronic Toll Collection } \\
\text { capabilities }\end{array}$ & Input \\
\hline $\bar{X} 19$ & $\begin{array}{l}\% \text { of Fixed route buses equipped with Automatic Vehicle } \\
\text { Location (AVL) }\end{array}$ & Input \\
\hline $\mathrm{X} 20$ & $\begin{array}{l}\% \text { of Demand responsive vehicles that operate under Computer } \\
\text { Aided Dispatch (CAD) }\end{array}$ & Input \\
\hline $\mathrm{X} 21$ & $\begin{array}{l}\% \text { of Fixed route buses with electronic real-time monitoring of } \\
\text { system components }\end{array}$ & Input \\
\hline $\mathrm{X} 22$ & $\%$ of Vehicles under Computer Aided Dispatch (CAD) for EM & Input \\
\hline $\mathrm{X} 23$ & $\begin{array}{l}\% \text { of Vehicles equipped with on-board navigation capabilitics for } \\
\text { EM (Emergency Management) }\end{array}$ & Input \\
\hline $\mathrm{X} 24$ & $\%$ of Highway rail intersections under electronic surveillance & Input \\
\hline Y1 & Average concentration of $\mathrm{CO}$ in ppm over an area & Target \\
\hline $\mathrm{Y} 2$ & Average concentration of $\mathrm{NO}_{2}$ in ppm over an area & Target \\
\hline $\mathrm{Y} 3$ & Average concentration of $\mathrm{O}_{3}$ in ppm over an area & Target \\
\hline
\end{tabular}




\section{CHAPTER IV}

\section{RESEARCH METHODOLOGY}

Methodology refers to more than simply a set of methods; rather it refers to the rationale and the philosophical assumptions that underlie the particular study. Often there are different methods that may be suitable to investigate a research problem. The methodology defines reasons why a particular method or procedure is chosen. The research methods must be appropriate to meet the study objectives. The methodology should also discuss anticipated challenges and explain actions taken to address them, mitigate difficulties, and provide guidance for any expected issues and minimize adverse impacts. In some cases, it is useful for researchers to adapt or replicate methodology used in related or similar studies, so sufficient information is often provided in technical publications to allow others to duplicate the work. This is particularly the case when a new method is developed, or an innovative adaptation used (Language Center, AIT, 2008).

To achieve the research objectives defined in chapter I, a set of specific methods/procedures was developed. The methods and the rationale of using these methods are explained in the following sections.

\subsection{Data Normalization}

In many numerical methods and model applications, data preparation techniques are essential to organize and condition the raw field observations or measurements prior 
to use. This is the case in most artificial neural network (ANN) model applications where data normalization with specific constraints or criteria, is crucial to prepare data for application as well as to significantly improve the efficiency of the model operation (Sola and Sevilla, 1997). Specifically in ANN applications, the process of back propagation used for neural network training or calibration requires all training targets to be normalized to the range between 0 and 1 . This is due to the output node's signal, commonly in the form of a sigmoidal activation function, being restricted to the 0 to 1 range. For consistency in the calibration and application procedures, all data values for both input and target (output) variables were scaled to the 0 to 1 range. For a set of training data that may naturally fall between the limits 0 and 1 , normalization may still be desirable. For example, if target data are between 0.1 and 0.2 , it would be better to normalize the data over a wider range to provide a broader spectrum for model training resolution and improved prediction over an optimal range (Chao and Skibniewski, 1998). Additionally, a skewed form of normalization may be used in order to address the distribution of data density. In this case data values may occur over a broad range of values, yet may be concentrated over a more narrow range - and this property may be exploited in normalization to provide a spreading or distribution of the data more evenly across the normalized spectrum.

In this research, two methods were used to normalize the input and target data. Reason for using two methods was to highlight the importance of data normalization for the application of neural networks to complex engineering problems. It was subsequently established that the method of data normalization affects the model prediction performance for a given set of input variables and training data, and one may obtain 
different results for the same set of training facts. The two normalization methods are described in the following sections.

\subsubsection{Normalization method one (n1)}

The first normalization method is termed as $\mathrm{nl}$ in this dissertation. The method of normalizing the data can be summarized in the following steps:

The normalized value of any variable data point is set equal to the value of that data point under consideration scaled by the maximum value (observed or expected or possible) of the variable.

For example, if data are recorded with the following magnitudes: $0.2,0.3,1.2,1.4$, $\& 2.5$; the maximum data value is 2.5 and the corresponding respective normalized values of each data point for use in the artificial neural network training are as follows:

Normalized value of $0.2=(0.2 / 2.5)=0.08$,

Normalized value of $0.3=(0.3 / 2.5)=0.12$,

Normalized value of $1.2=(1.2 / 2.5)=0.48$,

Normalized value of $1.4=(1.4 / 2.5)=0.56$,

Normalized value of $2.5=(2.5 / 2.5)=1.00$.

\subsubsection{Normalization method two (n2)}

The second normalization method is termed as $\mathrm{n} 2$ in this dissertation. The method of normalizing the data can be summarized in the following steps:

Normalized value of any variable data point $=($ Value of that data point under consideration - Minimum value of the variable)/(Maximum value of the variable Minimum value of the variable) 
Taking the same example as in previous section, the respective normalized values by the second method are as follows:

Normalized value of $0.2=(0.2-0.2) /(2.5-0.2)=0.000$

Normalized value of $0.3=(0.3-0.2) /(2.5-0.2)=0.043$

Normalized value of $1.2=(1.2-0.2) /(2.5-0.2)=0.435$

Normalized value of $1.4=(1.4-0.2) /(2.5-0.2)=0.522$

Normalized value of $2.5=(2.5-0.2) /(2.5-0.2)=1.000$

It is important now to compare the both these methods. First method (n1) provides a narrower range as compared with that obtained by second method (n2). Range of normalized data values was from a minimum value to 1.00 for first method and it was from 0.00 to 1.00 for the second method. But it can not be stated with certainty which method is better.

The effect of the normalizing methods on model training or calibration and model application or validation results as reflected in performance criteria including correlation coefficients are presented later in the results chapter.

\subsection{Reduction of ITS Application Variables}

This research compiled data records from ITS applications and consists of seventeen variables. As a component of the data reduction process this work required the development of a method to convert the seventeen ITS related variables into a single variable. This reduction was necessitated for the two reasons: first, an individual ITS application might reduce emissions significantly, yet this reduction may not be sufficient to be obscrvable by the available means in the context of overall air quality, analogous to "below detection limit" in environmental measurements, because the overall air quality is 
the result of emissions from all the transportation, industrial and natural activities in an urbanized area. Second, in this research, an attempt was made to highlight the effect of overall ITS (rather than individual system components) on air quality, negative or positive, to explain, justify, and validate the value of the overall systems environmental benefits.

\subsubsection{Correlations}

As a means of identifying any suspected non-parametric relationships between ITS applications and air quality variables, the Kendall tau $(\tau)$ rank correlation coefficient was used to define the degree of correspondence between the two since this best depicts the degree of association for non-parametric data (Wilkie, 1980). The Kendall's correlations between target variables and ITS application variables were computed using software available from Wcssa.net (URL: http://www.wessa.net/rwasp_kendall.wasp). As a verification of the normalization procedures, the correlation magnitudes were not affected by the method of normalization since both input and corresponding output variables were scaled with consistent methods. The results are shown in Table 4.1 and indicate correlation magnitudes between the variables for the normalized data under method one (n1) are identical to those normalized by method two (n2). Variable X1 through X7 are transportation/highway related and variables $\mathrm{Y} 1, \mathrm{Y} 2$, and $\mathrm{Y} 3$ are target/output variables as mentioned in Chapter-III. Correlations for X1 through X7 were not found because these were not needed. Correlations for ITS related variables from X8 to X24 were calculated since these were used to convert the seventeen ITS related variables into a single variable. Most of the correlations are having low values indicating a weak relation between an ITS application and air quality and that is true up to some extent in the sense 
that an individual ITS application really does not affect the overall air quality much. Correlation coefficient calculated shown in Table 4.1 have low numerical values showing weak relationships; still they provide a basis to convert the seventeen ITS variables into one. A weak relationship is still a relationship, however weak it may be.

Table 4.1 Correlations

\begin{tabular}{|c|c|c|c|}
\hline \multirow[t]{2}{*}{ ITS Variables } & \multicolumn{3}{|c|}{ Target Variables } \\
\hline & Y1 $(\mathrm{CO})$ & Y2 ( & Y3 \\
\hline $\mathrm{X8}$ & -0.1318199 & 0.1878397 & 0.0315266 \\
\hline X9 & -0.0298406 & -0.0241524 & -0.0378799 \\
\hline $\mathbf{X 1 0}$ & -0.0486612 & 0.0599820 & -0.0877814 \\
\hline X11 & 0.0767697 & -0.0789142 & -0.0266254 \\
\hline $\mathrm{X} 12$ & 0.0625569 & 0.1088283 & -0.0498106 \\
\hline $\mathbf{X 1 3}$ & 0.0637094 & 0.0601325 & -0.0798940 \\
\hline X14 & 0.0228942 & 0.1830461 & -0.0011008 \\
\hline $\mathrm{X} 15$ & 0.0205741 & 0.0114823 & $-0.0964 \overline{10}$ \\
\hline X16 & 0.2096858 & -0.0555733 & -0.0360316 \\
\hline $\mathbf{X 1 7}$ & -0.0224172 & 0.2454934 & 0.1173083 \\
\hline $\mathrm{X} 18$ & -0.1577866 & 0.0698830 & -0.0551721 \\
\hline X19 & -0.1099675 & 0.0571351 & -0.1569653 \\
\hline $\mathbf{X 2 0}$ & -0.0702035 & 0.0923930 & -0.0416617 \\
\hline $\mathbf{X 2 1}$ & -0.1445423 & 0.0387003 & -0.1614164 \\
\hline
\end{tabular}




\begin{tabular}{|l|l|l|l|}
\hline $\mathbf{X 2 2}$ & -0.0092677 & -0.0380587 & 0.0101071 \\
\hline $\mathbf{X 2 3}$ & -0.1079034 & -0.0064517 & -0.0086043 \\
\hline $\mathbf{X 2 4}$ & 0.0457885 & 0.0615584 & -0.1233637 \\
\hline
\end{tabular}

\subsubsection{Conversion of seventeen ITS variables into one}

ITS applications affect the air quality in varying degrees. Some ITS applications might not be affecting the air quality significantly as is evident by low values of correlation coefficients and some might be affecting it significantly as is indicated by their high values of correlation coefficients. Importance to an ITS application, therefore, should be assigned in proportion of its corrclation coefficient. Absolute value of correlation coefficient is taken as the basis to give weight to an ITS application intensity data and it is only a way to assign degree of importance to a particular ITS application. The seventeen ITS variables were assigned weights in proportion of the absolute values of their correlation coefficients. Each ITS variable (normalized) was multiplied by the respective correlation coefficient (absolute values), the values were summed, and the result was defined as a new single ITS variable (weighted). Subsequently, this ITS (weighted) was normalized by both the methods of normalization and re-named as ITS$\mathrm{nl}$ and ITS-n2 respectively. Weight for each target variable $-\mathrm{Y} 1, \mathrm{Y} 2$, and $\mathrm{Y} 3=$ Absolute value of correlation coefficient.

For example,

New York-Newark urbanized area's seventeen ITS data values were: $\mathrm{X} 8=0.27$, $\mathrm{X} 9=0.22, \mathrm{X} 10=0.24, \mathrm{X} 11=0.51, \mathrm{X} 12-0.149, \mathrm{X} 13=0.0923, \mathrm{X} 14-0.23, \mathrm{X} 15=0.18$, 
$\mathrm{X} 16=0.48, \mathrm{X} 17=0.0555, \mathrm{X} 18=0.71, \mathrm{X} 19=0.21, \mathrm{X} 20=0.18, \mathrm{X} 21=0.03, \mathrm{X} 22=0.27$, $\mathrm{X} 23=0.0149$, and $\mathrm{X} 24=0.82$

Absolute values of correlation coefficients between the ITS variables and ambient air concentration of $\mathrm{CO}$ were: $0.13182,0.029841,0.048661,0.07677,0.062557$, $0.063709,0.022894,0.020574,0.209686,0.022417,0.157787,0.109968,0.070204$ $0.144542,0.009268,0.107903$, and 0.045789 respectively.

Therefore, ITS $($ weighted $)=(0.27)(0.13182)+(0.22)(0.029841)+$ $(0.24)(0.048661)+(0.51)(0.07677)+(0.149)(0.062557)+(0.0923)(0.063709)+$ $(0.23)(0.022894)+(0.18)(0.020574)+(0.48)(0.209686)+(0.0555)(0.022417)+$ $(0.71)(0.157787)+(0.21)(0.109968)+(0.18)(0.070204)+(0.03)(0.144542)+$ $(0.27)(0.009268)+(0.0149)(0.107903)+(0.82)(0.045789)$ $=0.404419874=40.4 \%$

Maximum Value $=0.846354911=84.6 \%$

Therefore, ITS-n1 $=0.404419874 / 0.846354911=0.477837216$

Using a similar procedure for normalization method $\mathrm{n} 2$, the resulting variable is, ITS-n2 $=0.45299535$

Hence, this methodology converted the seventeen ITS variables into a single summary variable. It is definitely a better way than "averaging" in which average intensity of ITS application could have been used in the model.

In the following section, the revised list of variables after reduction is shown in Table 4.2. These variables are those used in the ANN and Multiple Regression models. 
Table 4.2 Revised Variables

\begin{tabular}{|c|l|l|}
\hline $\begin{array}{c}\text { Designation of } \\
\text { Variable }\end{array}$ & & Type \\
\hline $\mathrm{X} 1$ & Population in thousands & \\
\hline $\mathrm{X} 2$ & Road Length in miles & Input* \\
\hline $\mathrm{X} 3$ & Daily Vehicle Miles Traveled (DVMT) in thousands & Input \\
\hline $\mathrm{X} 4$ & Land Area in square miles & Input \\
\hline $\mathrm{X} 5$ & Freeway DVMT in thousands & Input \\
\hline $\mathrm{X} 6$ & Freeway Miles & Input \\
\hline $\mathrm{X} 7$ & Annual average daily traffic on freeways & Input \\
\hline $\mathrm{X}$ '8 & \% ITS (converted or reduced) in an area & Input \\
\hline Y1 & Average concentration of $\mathrm{CO}$ in ppm over an area & Target* \\
\hline $\mathrm{Y} 2$ & Average concentration of $\mathrm{NO}_{2}$ in ppm over an area & Target \\
\hline Y3 & Average concentration of $\mathrm{O}_{3}$ in ppm over an area & Target \\
\hline
\end{tabular}

*Input variables are independent variables while target variables are dependent variables.

\subsection{Modeling}

Engineering and scientific modeling is the process of generating abstract, conceptual, graphical and/or mathematical models, and physical geometrically-scaled models to study a system of interest. The modeling process refers to generating a model 
as a conceptual representation of the phenomenon of interest as a physical, mathematical, or logical representation of a system of entities, phenomena, or processes. Basically a model may be a simplified abstract view of the complex reality (Silvert, 2001), allowing for controlled, reproducible, repeatable, and measureable study and analysis of the system. In this work a computational mathematical model is selected and developed to study the relation and impact of ITS on operationally measured environmental factors and indictors of environmental impacts in urban transportation network systems.

\subsubsection{ANN Modeling}

In this research, the relation between ITS components and improvements in measureable environmental parameters was studied. To extract quantitative measures of this abstract relationship, a method capable of mapping the complex non-linear and unknown degree of freedom relationships between independent and dependent variables was researched. The method must also be capable of adapting to the available complex and noisy operational data representing the relationships between input and output variables. Artificial neural network models can provide such a model framework since these are designed to self-adjust within the model structure through the training process and extract the relationship from the examples shown in input-output data patterns. Owing to the combined effect of the multi-layer structure and the non-linear activation function, ANN models can represent relations that are difficult to describe using conventional mathematical functions. It has been proven that many arbitrary irregular patterns can be mapped by a neural network with two hidden layers and thus this 
adaptability is useful for developing the type of mapping function required for this work (Pao, 1989).

As can be seen from Table 4.2, X1 through $\mathrm{X}^{\prime} 8$ are the eight independent variables and $\mathrm{Y} 1, \mathrm{Y} 2$, and $\mathrm{Y} 3$ are the dependent variables. Since the relationship between dependent and independent variable is not known (appears to be non-linear), ANN therefore turns out to be well-suited for modeling the relationship.

\subsubsection{Feedforward Neural Networks}

Feedforward artificial neural networks were used for modeling in this research since these are the most parsimonious form, most commonly applied with the back propagation training algorithm, and are widely used in several civil engineering applications (Lingireddy and Ormsbce, 1998). The model structure consists of a (possibly large) number of simple neuron-like processing units, organized in layers. Every neuron unit in a layer is connected with all the units in the adjacent layers. The strength of the layer-to-layer neuron connections is not equal; each connection typically has a different strength or inter-connection weight. The weights on the connections encode the knowledge of a network or pattern "learned" in the training or calibration process (Lingireddy and Ormsbee, 1998). Often the units in a neural network are also called nodes.

In an operational or applied mode, the ANN model accepts data entry at the input nodes and passes through the network, layer by layer, undergoing computational modification, until it is transformed and arrives at the output layer in the desired form or 
signal. Once calibrated or trained, and during normal operation, there is no feedback between layers. This is why the ANNs are called feed-forward neural networks. Prior to operational use, the ANN must be trained, and the back-propagation algorithm is one such training method using a gradient decent method for error minimization (Rumelhart et al., 1986). Starting with an assumed or specified set of initial weights for the connections, the back-propagation algorithm computes the error between the predicted and specified target values at the output layer. If this error is greater than a prescribed tolerance, the algorithm updates the connection weights by iteratively back-propagating the error using a gradient decent method. The software system MATLAB 7.0 was used in this study (MATLAB, 7.0, 2004).

\subsubsection{Network Architecture}

As briefly described above, artificial neural network models are composed of a set of nodes or neurons, joined together by connection weights or synapses. Each neuron performs a simple computational task, generally a basic yes/no decision. The synapses are the connections that link neurons together, from layer to layer, and re-scale or adjust the neuron response by weighting the response as the signal passes to the next layer. This system eventually links input values to produce the output signals.

In programming terms, a synapse is an object which links one neuron connected to its input to another connected to its output. A neuron is a slightly more complex object which can be connected to one or more input synapses and one or more output synapses. The structure of any neural network is therefore defined by the way in which various neurons and synapses are linked together. Selecting the number of hidden layers, the 
number of neurons or nodes in each layer, the learning rule, the activation function (or transfer function) and the level of error tolerance is the process of searching for suitable/optimum architecture of ANN (Howard et al., 2006). Given below is an example of setting up architecture for an ANN:

- MATLAB code used for setting up the architecture was: net=newff(minmax $(\mathbf{p}),[3,2,1],\{$ 'tansig','tansig','purelin'\},'trainlm'); where $\mathrm{p}$ is the input variables (independent), $[3,2,1]$ indicates the number of nodes in first hidden layer, second hidden layer, and output layer respectively.

- $\{$ 'tansig','tansig','purelin'\} in the code indicates the transfer functions in first hidden layer, second hidden layer, and output layer respectively.

- Trainlm in the code indicates the L.M.learning (or training) rule.

- ff indicates the feed-forward network.

- [net,tr]=train(net,p,t); code was used for training, where $\mathbf{t}$ indicates the target variable (dependent).

- Error tolerance was kept 0.0002 and epochs were restricted upto 5000 .

- $\operatorname{tr1=sim(net,p);~code~was~used~for~simulation~where~tr1~represents~}$ simulated results when input is $\mathrm{p}$ in a trained network.

\subsubsection{Training Algorithm (Learning Rule)}

A learning rule is defined as a procedure for modifying the weights and biases of a nctwork. This procedure can also be referred to as a training algorithm. The learning rule is applied to train the network to perform some particular task. In supervised learning that 
has been used in this research, the learning rule is provided with a set of examples (the training sets) of proper network behavior. As the inputs are applied to the network, the network outputs are compared to the targets. The learning rule is then used to adjust the weights and biases of the network in order to move the network outputs closer to the targets (Howard et al., 2006).

It is very difficult to know which training algorithm will be the fastest for a given problem. It depends on many factors, including the complexity of the problem, the number of data points in the training set, the number of weights and biases in the network, the error goal, and whether the network is being used for pattern recognition or function approximation. However, the Levenberg-Marquardt (LM) training algorithm has been used in this research since it is best suited for function approximation (regression) and converges very fast when data set are less in number comparatively (Howard et al., 2006).

\subsubsection{Transfer Function (Activation Function)}

One of the important components in determining a neuron's response is called the transfer function. The transfer function describes how a neuron's firing rate varies with the input it receives. A very sensitive neuron may fire with very little input, for example. A neuron may have a threshold, and fire rarely below threshold, and vigorously above it. A neuron may have a bell-curve style firing pattern, increasing its firing rate up to a maximum, and then leveling off or decreasing when over-stimulated. A neuron may sum its inputs, or average them, or something entirely more complicated. Each of these behaviors can be represented mathematically, and that representation is called the transfer function. In simple language, it can be said that Transfer function connects input to 
output. These functions for the hidden units are needed to introduce nonlinearity into the network. Without nonlinearity, hidden units would not make nets more powerful than just plain perceptrons (which do not have any hidden units, just input and output units). The reason is that a linear function of linear functions is again a linear function. However, it is the nonlinearity — the capability to represent nonlinear functions-that makes multilayer networks so powerful. Almost any nonlinear function does the job, except for polynomials. For backpropagation learning, the activation function must be differentiable, and it helps if the function is bounded; the sigmoidal functions such as logistic and tanh and the Gaussian function are the most common choices. Functions such as tanh or arctan that produce both positive and negative values tend to yield faster training than functions that produce only positive values such as logistic, because of better numerical conditioning. For hidden units, sigmoid activation functions are usually preferable to threshold activation functions. Networks with threshold units are difficult to train because the error function is stepwise constant, hence the gradient either does not exist or is zero, making it impossible to use backprop or more efficient gradient-based training methods. With sigmoid units, a small change in the weights will usually produce a change in the outputs, which makes it possible to tell whether that change in the weights is good or bad. With threshold units, a small change in the weights will often produce no change in the outputs (Howard et al., 2006).

In this research, sigmoid function was used in hidden layers followed by linear function in output layer. Multiple layers of neurons with nonlinear transfer functions allow the network to learn nonlinear and linear relationship between input and output 
vectors. The linear output layer lets the network produce values outside the range -1 to +1 (Howard et al., 2006).

\subsubsection{Multiple Linear regression Modeling}

The general purpose of multiple regressions is to learn more about the relationship between several independent or predictor variables and a dependent or criterion variable. Matlab was used for this purpose in this research and 10-fold cross-validation technique was utilized to find the predicted values of dependent variables-Y1, Y2, and $\mathrm{Y} 3-$ so that ANN model prediction could be compared with Multiple Linear Regression Model prediction and superiority of ANN modeling could be estabished. Limitation of this modeling was that it was assumed that the relationship between variables was linear. In practice this assumption can virtually never be confirmed; fortunately, multiple regression procedures are not greatly affected by minor deviations from this assumption (Draper \& Smith, 1998).

\subsubsection{K-fold Cross-validation}

In $K$-fold cross-validation, the original sample is partitioned into $K$ subsamples. Of the $K$ subsamples, a single subsample is retained as the validation data for testing the model, and the remaining $K-1$ subsamples are used as training data. The crossvalidation process is then repeated $K$ times (the folds), with each of the $K$ subsamples used exactly once as the validation data. The $\mathrm{K}$ results from the folds then can be averaged (or otherwise combined) to produce a single estimation. The advantage of this method over repeated random sub-sampling is that all observations are used for both 
training and validation, and each observation is used for validation exactly once (Kohavi, 1995). 10-fold cross-validation is commonly used and the same has been used in this research as well.

\subsection{Prediction/forecasting}

As 10 -fold cross validation technique was used, the original data (X1 to X'8 and $\mathrm{Y} 1, \mathrm{Y} 2, \& \mathrm{Y} 3)$ were partitioned in 10 subsets. Of the 10 subsets, a single subset was retained as the validation data for testing both the models (ANN and MR), and the remaining 9 subsets were used as training data. The cross-validation process was then repeated 10 times, with each of the 10 subsets used exactly once as the validation data. The 10 results from the folds then were compared with the actual target variables' values (Y1, Y2, and Y3) and errors in percent were calculated for each data point. The Mean Absolute Error (MAE) in prediction was found by averaging the absolute values of percent errors for all data points.

The data points with large errors (outliers) were identified and were removed from the data set for further refinement and the refined data set, now named as "modified data", once again went through the same process as mentioned above to check if it improved the prediction results.

\subsubsection{Removal of Outliers}

In statistics, an outlier is an observation that is numerically distant from the rest of the data. Results derived from data sets that include outliers may be misleading. In most larger samplings of data, some data points will be further away from the sample mean than what is deemed reasonable. This can be due to incidental systematic error or it can simply be the case that some observations happen to be a long way from the center of the 
data. In this research certain data points produced large errors and were suspected to be outliers. For detecting outliers, a procedure was used which was as follows:

- First, mean was computed

- Second, standard deviation was computed

- Data points which were falling above or below 2 times the standard deviation from the mean were decided to be the outliers.

Original data points in this research corresponding to $\mathrm{CO}, \mathrm{NO}_{2}$, and $\mathrm{O}_{3}$ were 234,221 , and 236 and after removal of outliers, modified data points were 186, 196, and 217 respectively.

\subsubsection{ANN Modeling}

Neural networks can be explicitly programmed to perform a task by manually creating the topology and then setting the weights of each link and threshold. However, this by-passes one of the unique strengths of neural nets: the ability to program themselves. The most basic method of training a neural network is trial and error. If the network isn't behaving the way it should, change the topology/architecture of the NN. If the accuracy of the network declines, undo the change and make a different one. It takes time, but the trial and error method does produce results (Fraser, 2008). Choosing the right neural network topology to solve a particular problem with optimum generalization performance is not, in any case, a trivial problem.

The following were the salient features in ANN modeling adopted in this research for forecasting/predicting the target variables for a given set of input variables:

- First, very simple neural network topology with one hidden layer with one neuron was chosen and the MAE (in percent) was found. 
- Subsequently, the topologies were made more complex by increasing the number of nodes/neurons and the layers and the MAEs were found out.

- The topology/architecture that provided the least error was considered to be the best model for prediction/forecasting purpose.

- The same procedure was repeated for the modified data too.

- The procedure was completed for the data normalized by both the methods to see if method of normalization makes a difference in the results.

- Calculations were done to calculate the Mean Absolute Errors (MAEs) and Root Mean Square Errors (RMSEs).

- Curves were plotted between observed/predicted concentration v/s number of observations of a pollutant in ambient air for the best model to see if the trend of concentration variation for observed and predicted amongst a number of observations is same or not. If it was the same, then it would prove the model to be an accurate/robust one.

- Also scatter plots were made between observed $\mathrm{v} / \mathrm{s}$ predicted concentration to see if the most of the data were evenly and closely distributed about the 45 degree line.

- Above-mentioned curves were plotted for modified data also.

\subsubsection{Multiple Regression (MR) Modeling}

Multiple linear regression modeling was also utilized in this research to highlight the supremacy of ANN over MR in prediction/forecasting of targets. 10-fold crossvalidation technique was used in matlab platform. 
The following were the salient features in MR modeling adopted in this research for forccasting/predicting the target variables for a given set of input variables:

- Calculations were done to calculate the Mean Absolute Errors (MAEs) and Root Mean Square Errors (RMSEs).

- Curves were plotted between observed/predicted concentration v/s number of observations of a pollutant in ambient air for the MR model to see if the trend of concentration variation for observed and predicted amongst a number of observations is same or not.

- Also scatter plots were made between observed $\mathrm{v} / \mathrm{s}$ predicted concentration to see if the most of the data were evenly and closely distributed about the 45 degree line or not.

- Above-mentioned curves were plotted for modified data also.

\subsection{Effect of ITS on Ambient Air Quality}

Using a trained network to learn about the effect of a particular variable-ITS in this case - on air quality (concentration of $\mathrm{CO}, \mathrm{NO}_{2}$ or $\mathrm{O} 3$ in ambient air), involves running the network with all of the input ncurons, except the one of interest, held constant; interactions can be explored by setting input neurons at various values. Following procedure was adopted to find the effect of ITS (X'8 variable) on target variables (Y1, Y2, and $\mathrm{Y} 3)$ :

- First of all, the trained neural networks which provided the least MAEstotal four $\mathrm{NN}$, one each for a combination of method of normalization ( $\mathrm{n} 1$ and n2) and type of data (original or modified) - - were fed with the constant 
values of variables of $X 1$ through $X 7$ along with ITS values ( $\left.X^{\prime} 8\right)$ varying from 0 to 1.00 with a constant increment of 0.02 .

- Constant values of variables X1 through X7 that were fed to the model as mentioned in the previous section were the average values of these variables. For example, normalized average input values (by method-1, i.e.,n1) of $\mathrm{X} 1$ through $\mathrm{X} 7$ for $\mathrm{CO}$ prediction used were $0.121181,0.178049$, $0.178135,0.143888,0.174108$, and 0.418095 respectively while ITS (X'8 variable) values were $0.0,0.02,0.04,-----$ upto 1.00 .

- By putting these input values of X1 through X'8 in a trained Neural Network, predicted values of target variables could be found out and the curves were plotted between \% ITS and concentration of target variables to show how the intensity of ITS affected the ambient air quality.

- Similarly, by using MR modeling in the matlab platform, the same values of input variables were used to provide the values of the target variable, and these values were also included in the plot as mentioned in the previous point.

This was the methodology, as mentioned in earlier sections, adopted in this research that explained the methods and procedures used and their rationale. Also it explained the problems that were anticipated and the steps taken to prevent them from occurring. 


\section{CHAPTER V}

\section{RESULTS AND DISCUSSION}

To achieve the research objectives, a set of specific procedures/methods were used as described in Chapter IV and the results obtained are discussed in the following sections. The results have been categorized on the basis of target variables- $\mathrm{CO}, \mathrm{NO}_{2}$, and $\mathrm{O}_{3}$.and are presented in that order.

\subsection{Carbon monoxide (CO)}

Several configurations of ANN model structure (combinations of nodes and hidden layers) were implemented and applied using sets of the CO data--four sets altogether. These sets were as follows:

Set 1: Original data normalized by method-1 $(\mathrm{n} 1)$,

Set 2: Modified data normalized by method-1 (n1),

Set 3: Original data normalized by method-2 (n2), and

Set 4: Modified data normalized by method-2 (n2).

The notations $\mathrm{nl}$ and $\mathrm{n} 2$ indicate the two methods of data normalization as described in Chapter IV. 


\subsubsection{ANN Architecture selection}

\subsubsection{Results}

In this section, the influence of ANN architecture on the mean absolute error (MAE) performance measures has been highlighted. Starting with a nominal ANN architecture and subsequently increasing model structure complexity, the MAE performance measures were determined for applications corresponding to each model architecture or configuration. Also, a Multiple Regression study was performed on each of the four data sets and the corresponding MAE values were determined. Tables 5.1 through 5.4 summarize the model architecture configurations and corresponding MAEs for each. In Table 5.1 the first column defines the model architecture or structure using a compact notation in a 3-digit format. The three digits shown correspond to either the number of layers or nodes in each layer. The first digit denotes the number of hidden layers, the second digit denotes the number of nodes in first hidden layer, and the third digit denotes the number of nodes in second hidden layer. Tables 5.2 through 5.4 follow a similar form and present results corresponding to the remaining data sets. Additionally, Figures 5.1 through 5.4 provide a graphical summary and comparison of performance MAE measures for each model structure. Again, Figures 5.2 through 5.4 are presented in the same format as Figure 5.1 and present results for the remaining data sets. 
Table 5.1 ANN model performance according to architecture and corresponding MAE measure (for data normalized by method-1)

Where:

\begin{tabular}{|c|c|}
\hline Configuration-n1 & $\begin{array}{c}\% \mathrm{E} \\
\text { (MAE) }\end{array}$ \\
\hline$(1-1-0)^{*}$ & 21.7 \\
\hline$(1-2-0)$ & 26.3 \\
\hline$(1-3-0)$ & 22.8 \\
\hline$(1-4-0)$ & 20.9 \\
\hline$(1-5-0)$ & 24.4 \\
\hline$(2-2-2)$ & 20.4 \\
\hline$(2-2-3)$ & 20.5 \\
\hline$(2-3-3)$ & 17.2 \\
\hline $\begin{array}{c}\text { Multiple } \\
\text { Regression }\end{array}$ & 24.1 \\
\hline
\end{tabular}

Configuration- $\mathrm{n} 1=$ Architecture of the ANN model and $\mathrm{n} 1$ indicates the first method of normalization.

Configuration- $\mathrm{n} 2=$ Architecture of ANN and $\mathrm{n} 2$ indicates the second method of normalization.

Configuration- $\mathrm{n} 1-\mathrm{m}=$ Architecture of ANN and $\mathrm{nl}$ jndicates the first method of normalization, and ' $\mathrm{m}$ ' indicates the modified data.

Configuration- $\mathrm{n} 2-\mathrm{m}=$ Architecture of $\mathrm{ANN}$ and $\mathrm{n} 2$ indicates the second method of normalization, and ' $\mathrm{m}$ ' indicates the modified data.

$\% \mathrm{E}=$ Mean Absolute Error (MAE) expressed in percent format.

$(1-1-0)^{*}=$ first digit denotes the number of hidden layers, second digit denotes the number of nodes in first hidden layer, and third digit denotes the number of nodes in second hidden layer.

Multiple Regression $=$ Multiple Regression modeling. 
Mean Absolute Error (MAE) V/S ANN Architecture (for normalized data by method-1)

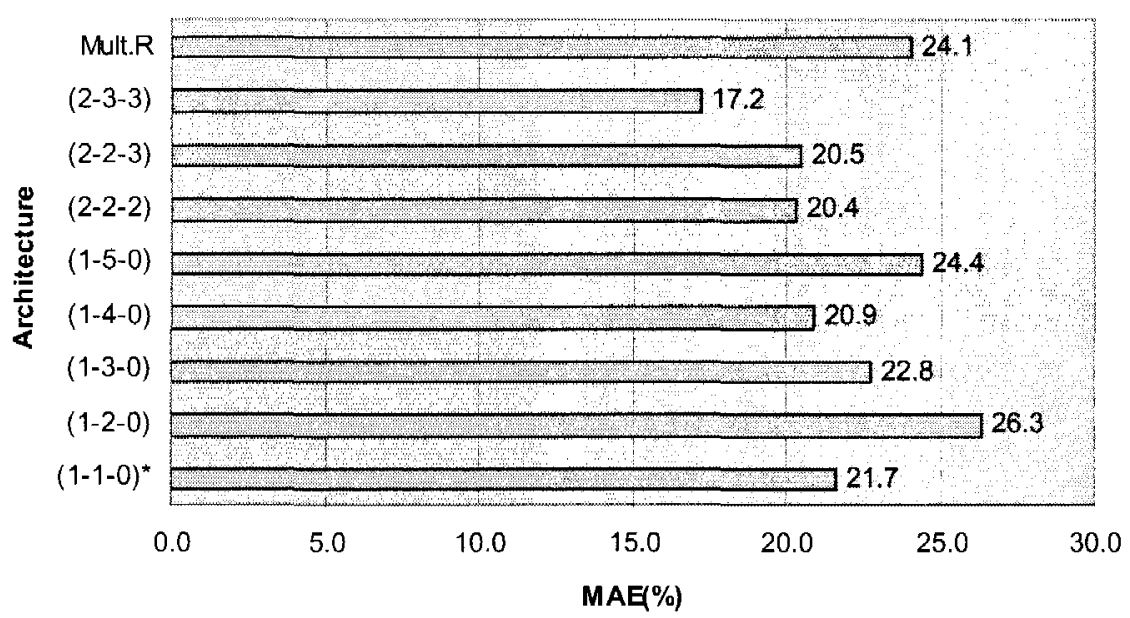

Figure 5.1

ANN performance summary according to architecture and corresponding

MAE (for data normalized by method-1) 
Table 5.2 ANN model performance according to architecture and corresponding

MAE measure (for modified data normalized by method-1)

\begin{tabular}{|c|c|}
\hline Configuration-n1-m & $\% \mathrm{E}$ (MAE) \\
\hline$(1-1-0)$ & 16.0 \\
\hline$(2-1-1)$ & 16.2 \\
\hline $\mathbf{( 2 - 2 - 1 )}$ & $\mathbf{1 4 . 0}$ \\
\hline$(2-3-3)$ & 19.2 \\
\hline Multiple Regression & 17.7 \\
\hline
\end{tabular}

Mean Absolute Error (MAE) V/S ANN Architecture (for modified normalized data by method-1)

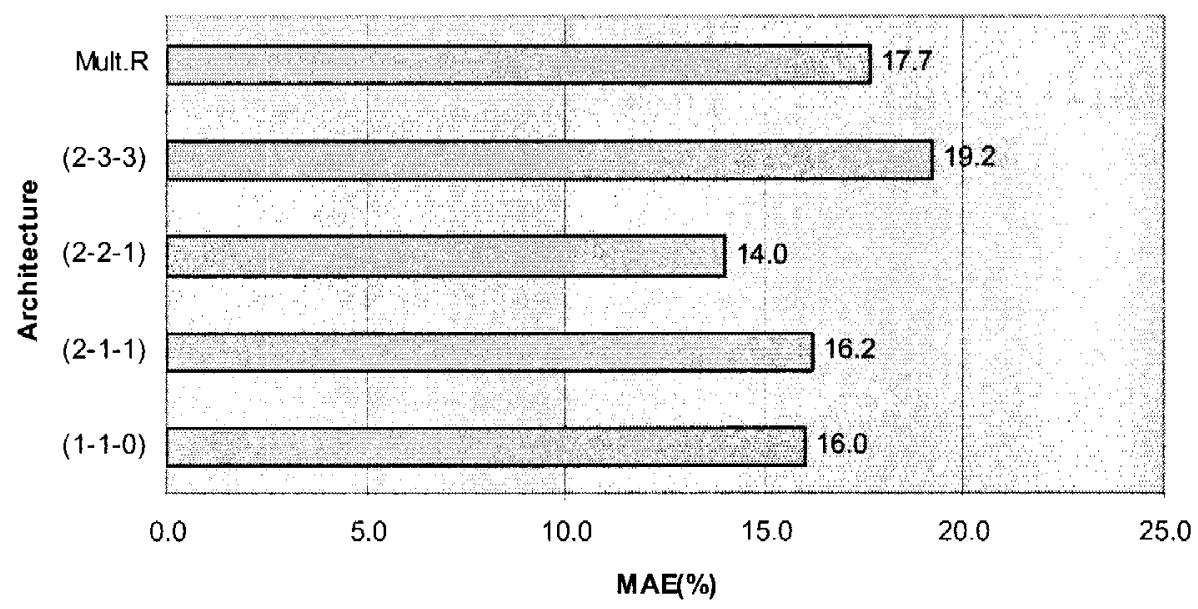

Figure 5.2

ANN performance summary according to architecture and corresponding MAE (for modified normalized data by method-1) 
Table 5.3 ANN model performance according to architecture and corresponding MAE measure (for data normalized by method-2)

\begin{tabular}{|c|c|}
\hline Configuration-n2 & $\% \mathrm{E}(\mathrm{MAE})$ \\
\hline$(1-1-0)$ & 20.5 \\
\hline$(1-3-0)$ & 27.0 \\
\hline$(2-1-1)$ & 20.2 \\
\hline$(2-2-2)$ & 20.0 \\
\hline$(2-3-2)$ & 22.3 \\
\hline$(2-3-3)$ & 21.6 \\
\hline$(2-4-4)$ & 19.8 \\
\hline$(2-5-5)$ & 27.7 \\
\hline Multiple Regression & 24.1 \\
\hline
\end{tabular}

Mean Absolute Error (MAE) VIS ANN Architecture (for normalized data by method-2)

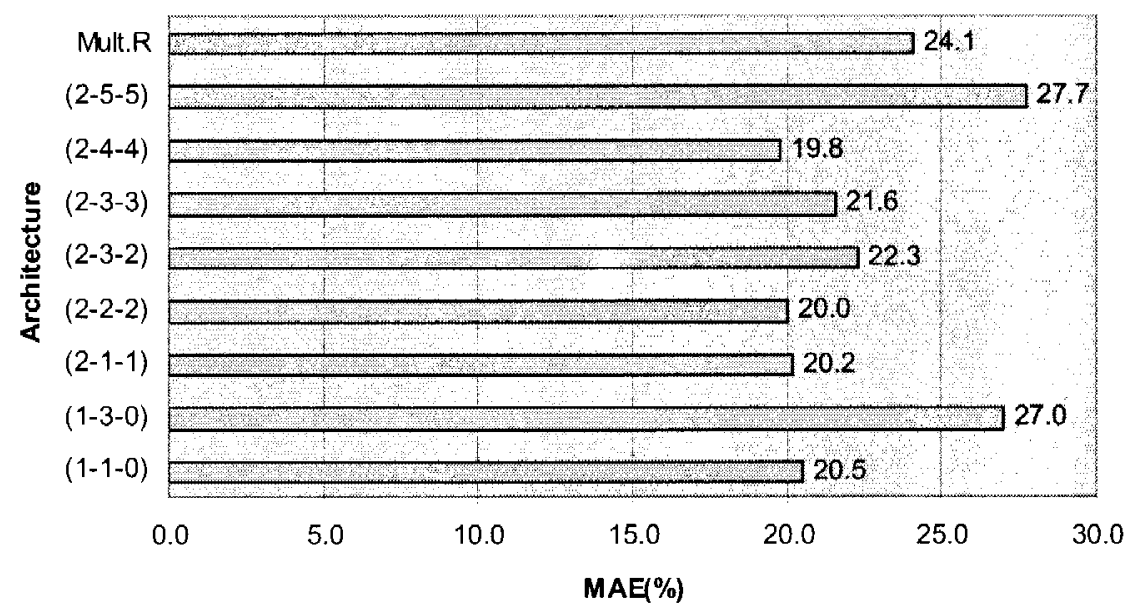

Figure 5.3

\section{ANN performance summary according to architecture and corresponding}

MAE (for normalized data by method-2) 
Table 5.4 ANN model performance according to architecture and corresponding MAE measure (for modified normalized data by method-2)

\begin{tabular}{|c|c|}
\hline Configuration-n2-m & $\% \mathrm{E}$ (MAE) \\
\hline$(1-1-0)$ & 16.0 \\
\hline$(1-2-0)$ & 15.1 \\
\hline$(1-3-0)$ & 15.0 \\
\hline$(2-1-1)$ & 16.2 \\
\hline$(2-2-2)$ & 14.5 \\
\hline$(2-3-3)$ & 16.8 \\
\hline$(2-2-1)$ & 22.2 \\
\hline$(2-1-2)$ & 14.8 \\
\hline Multiple Regression & 17.7 \\
\hline
\end{tabular}

Mean Absolute Error (MAE) V/S ANN Architecture (for modified normalized data by method-2)

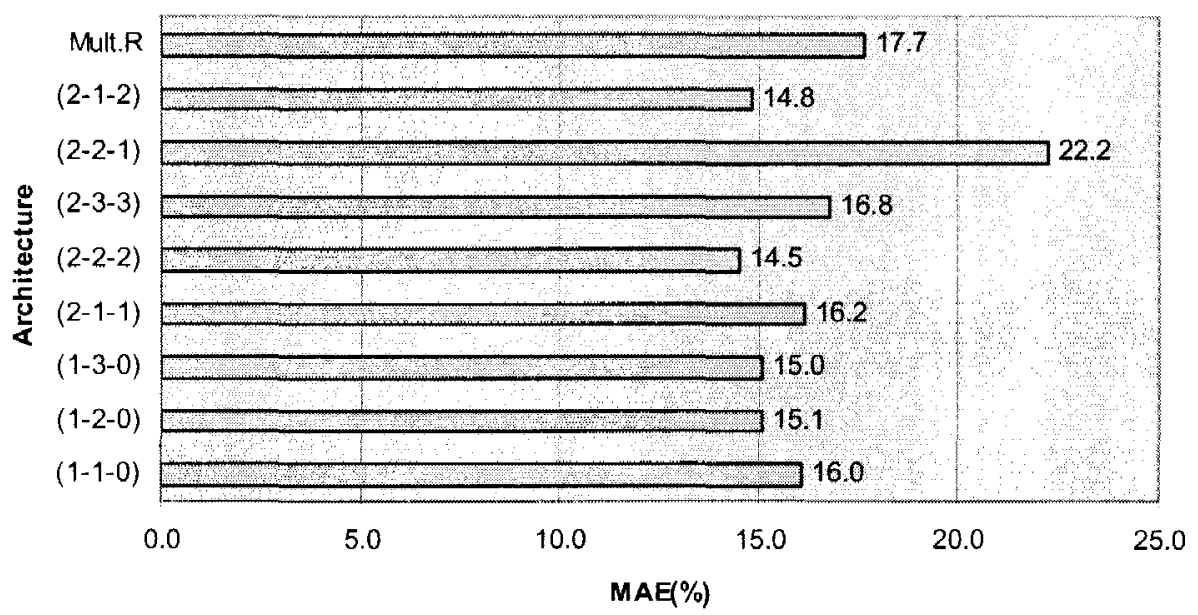

Figure 5.4

ANN performance summary according to architecture and corresponding MAE (for modified normalized data by method-2) 


\subsubsection{Discussion}

Several ANN model structure configurations or architectures were applied in order to arrive at the optimal form to minimize MAE. MAE is a standard performance measure to check the capability of a prediction model.

Entries in bold in the tables indicate the configuration of ANN with the lowest MAE in prediction. Following section summarizes the significant observations derived from the results of $\mathrm{CO}$ modeling with regard to variations in ANN Architecture.

- The values of MAE for data Set 1 varied from $17.2 \%$ corresponding to ANN model configuration (2-3-3) to $26.2 \%$ corresponding to ANN model configuration (1-2-0), and $24.1 \%$ for the multiple regression model.

- The MAE values for data Set 2 varied from $14.0 \%$ corresponding to ANN model configuration (2-2-1) to $19.2 \%$ for ANN model configuration (1-2$0)$, and $17.7 \%$ for the multiple regression model.

- The values of MAEs for Set 3 data varied from $19.8 \%$ corresponding to ANN model configuration (2-4-4) to $27.7 \%$ corresponding to ANN model configuration (2-5-5) and $24.1 \%$ for the multiple regression model.

- The values for MAEs for Set 4 data varied from $14.5 \%$ corresponding to ANN model configuration (2-2-2) to $22.2 \%$ corresponding to ANN model configuration (2-2-1) and $17.7 \%$ for the multiple regression model.

- The values for MAEs with Multiple Regression are same for Set 1 and Set 3 $(24.1 \%)$ and Set $2 \&$ Set $4(17.7 \%)$ indicating that the influence of the data normalization method does not significantly affect multiple regression modeling results. 
- From Table 5.1 through 5.4, it is evident that MAEs are larger when the ANN model is likely to be considered either under-trained or over-trained, however there are no clear-cut guidelines by which one can define the optimal configuration for ANN structure. Although the tendency is for more complex the relationships between dependent and independent variables require more complex ANN structures, the final details of model structure must still be determined using an iterative, trial and error style procedure. Through the training or model calibration process one must monitor the ANN evolution and avoid both under-trained and over-trained regions of the solution space.

- The MAE values in prediction applications vary over the range from $14 \%$ to $20 \%$ approximately and those are typical results considering the fact that the significant $\mathrm{CO}$ emissions from sources, in addition to the transportation factors, include industries, power plants etc. and those factors have not been included in this study due to the focus on transportation facilities.

- The lowest MAE value for Set 1 data was $17.2 \%$ and $14.0 \%$ for Set 2 (data modified by removing outliers indicating that by removing "outlicrs" the prediction error is reduced significantly. This same result was found for the data sets 3 and 4 , and for the multiple-regression model.

- The lowest MAE value for data Set 1 (data normalized by method-1, n1) was $17.2 \%$ and $19.8 \%$ for Set 3 (normalized by method-2, $\mathrm{n} 2$ ). Similarly, lowest MAE value for data Set 2 (normalized by method-1, n1) was $14.0 \%$ and $14.5 \%$ for Set 4 (normalized by method-2, $\mathrm{n} 2$ ). This result indicates 
the method of data normalization does significantly affect results with ANN modeling in this particular study; and method number one (n1) was found to provide a more accurate prediction relative to method two (n2).

\subsubsection{Comparison of Observed and Predicted values}

\subsubsection{Results}

The observed and predicted results from both the models--ANN model and Multiple Regression (MR) model — were compared using values for both the MAE and RMSE (for the CO parameter). The model configuration or architecture providing the least MAE result was selected as the "target" or optimal model and served to provide the predicted values for model validation evaluation. By using 10-fold technique, the predicted values were found for both the models, the optimal ANN and multiple regression. To provide a means for visualization of the results, a series of curves were plotted comparing observed and predicted values of CO concentration. Additionally, a series of scatter plots were developed to illustrate the statistical comparison relative to the perfect agreement line and provide an indication of model bias if any. Figures 5.5 through 5.12 depict the plots of observed and predicted for original data and Figures 5.13 through 5.20 show the plots between observed and predicted for modified data. 


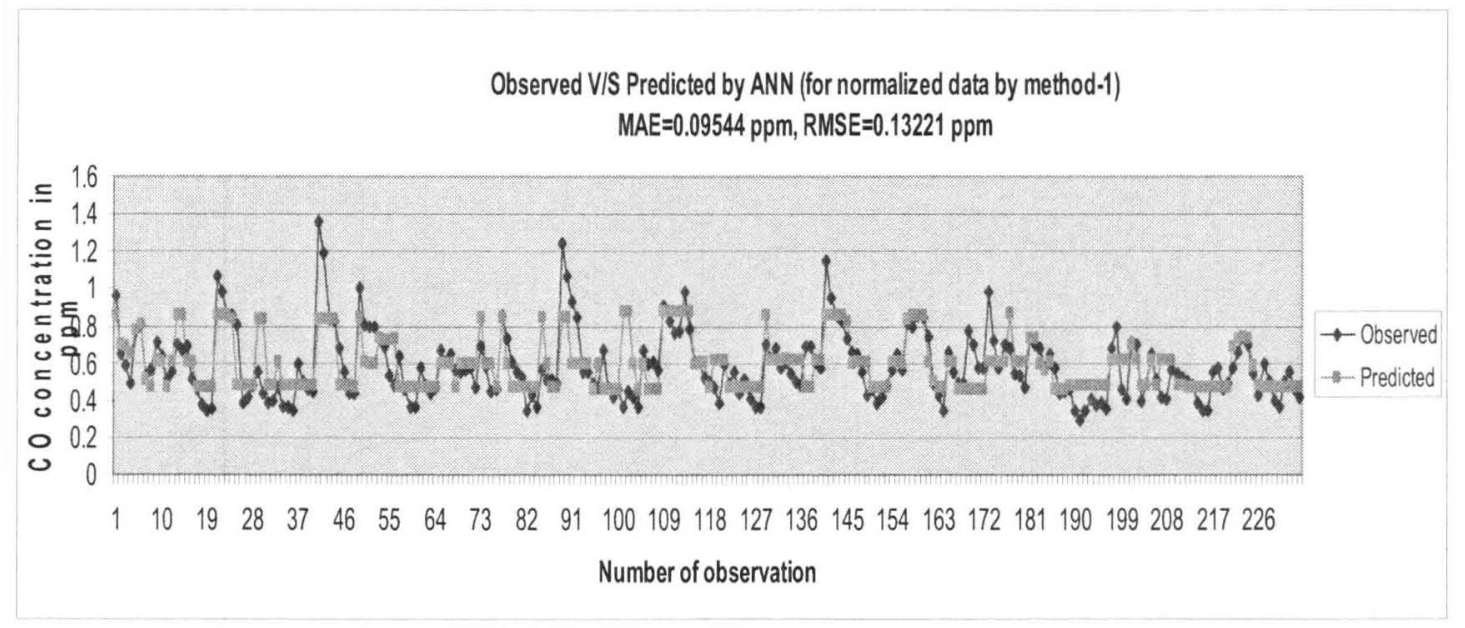

Figure 5.5

Observed versus Predicted values by ANN for normalized data by method-1

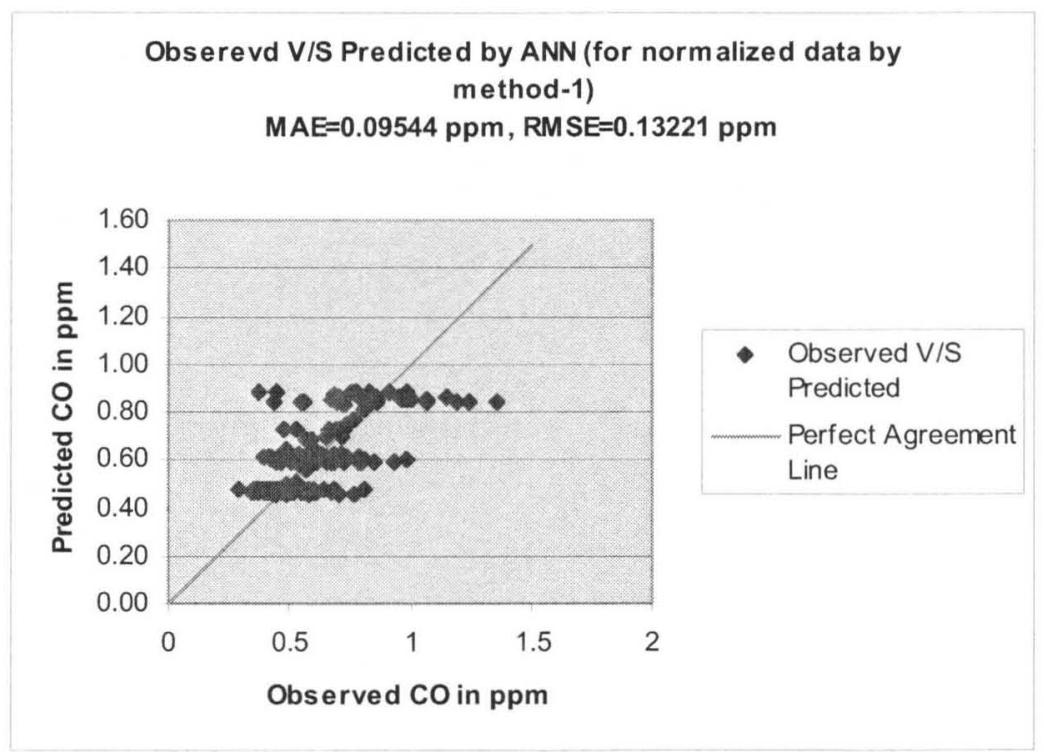

Figure 5.6

Observed and Predicted values by ANN for data normalized by method-1 
Observed VIS Predicted by ANN (for normalized data by method-2)

MAE $=0.11438 \mathrm{ppm}$, RMSE $=0.16866 \mathrm{ppm}$

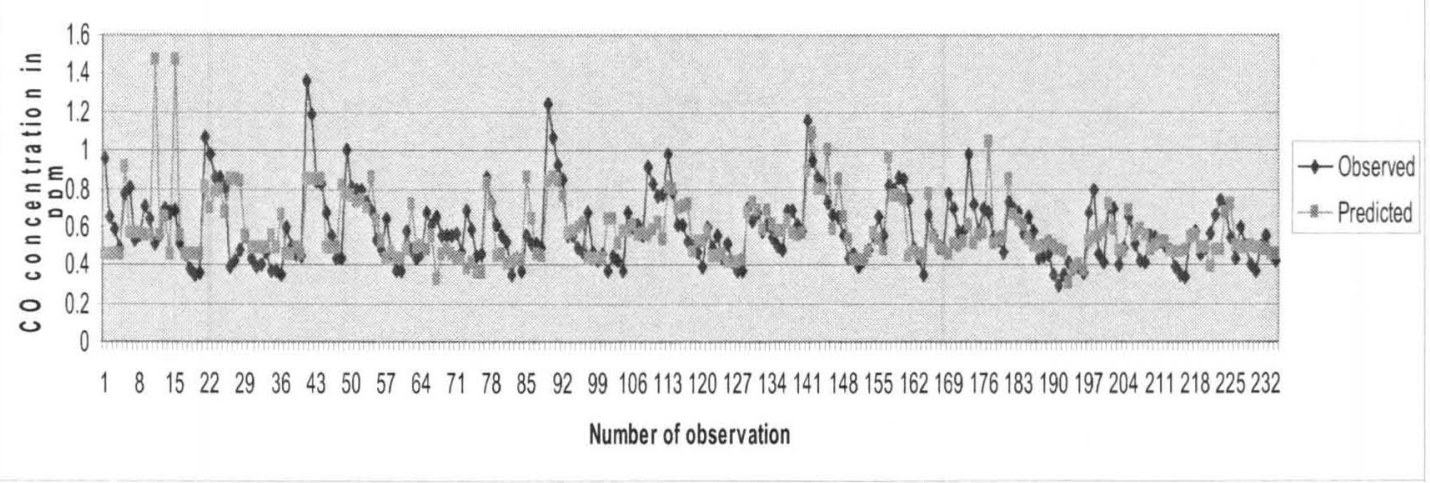

Figure 5.7

Observed versus Predicted values by ANN for normalized data by method-2

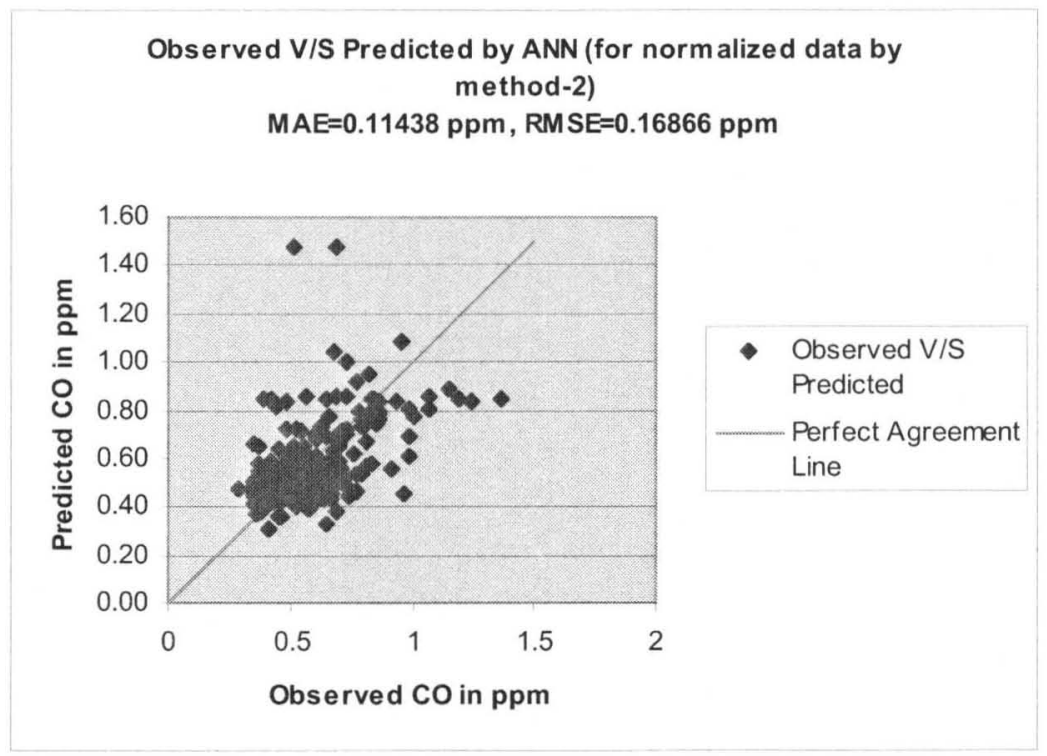

Figure 5.8

Observed versus Predicted values by ANN for normalized data by method-2 


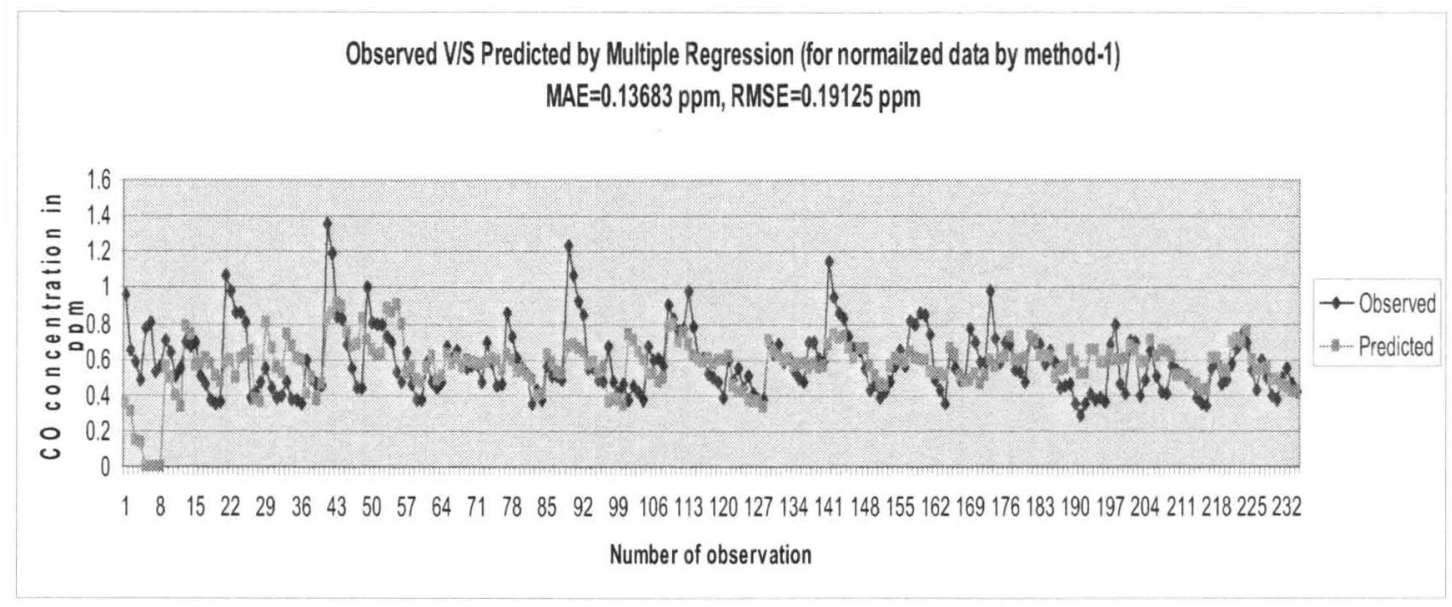

Figure 5.9

Observed versus Predicted values by MR model for normalized data by method-1

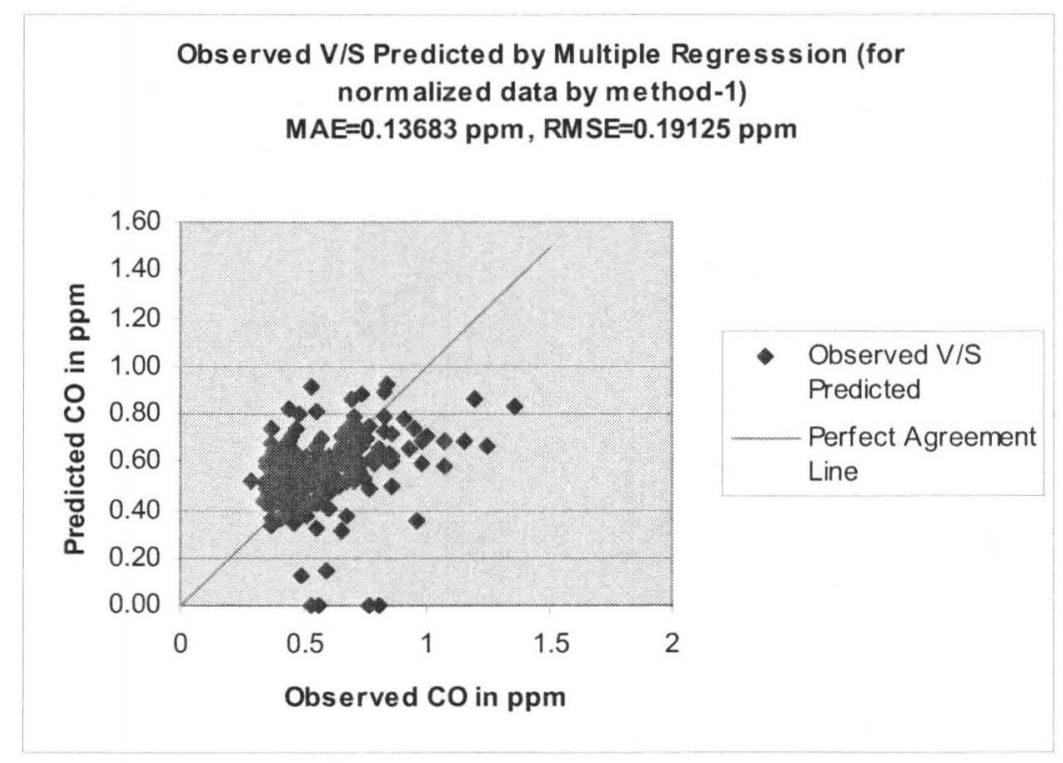

Figure 5.10

Observed versus Predicted values by MR model for normalized data by method-1 
Observed VIS Predicted by Multiple Regression (for normalized data by method-2)

MAE $=0.13683 \mathrm{ppm}$, RMSE $=0.19125 \mathrm{ppm}$

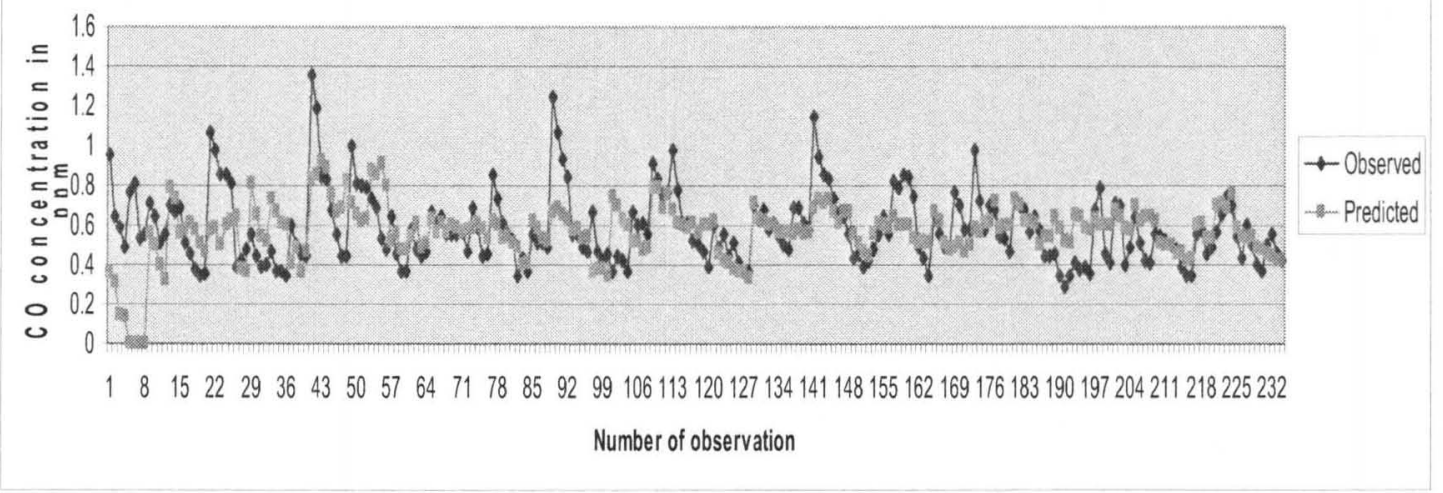

Figure 5.11

Observed versus Predicted values by MR model for normalized data by

method-2

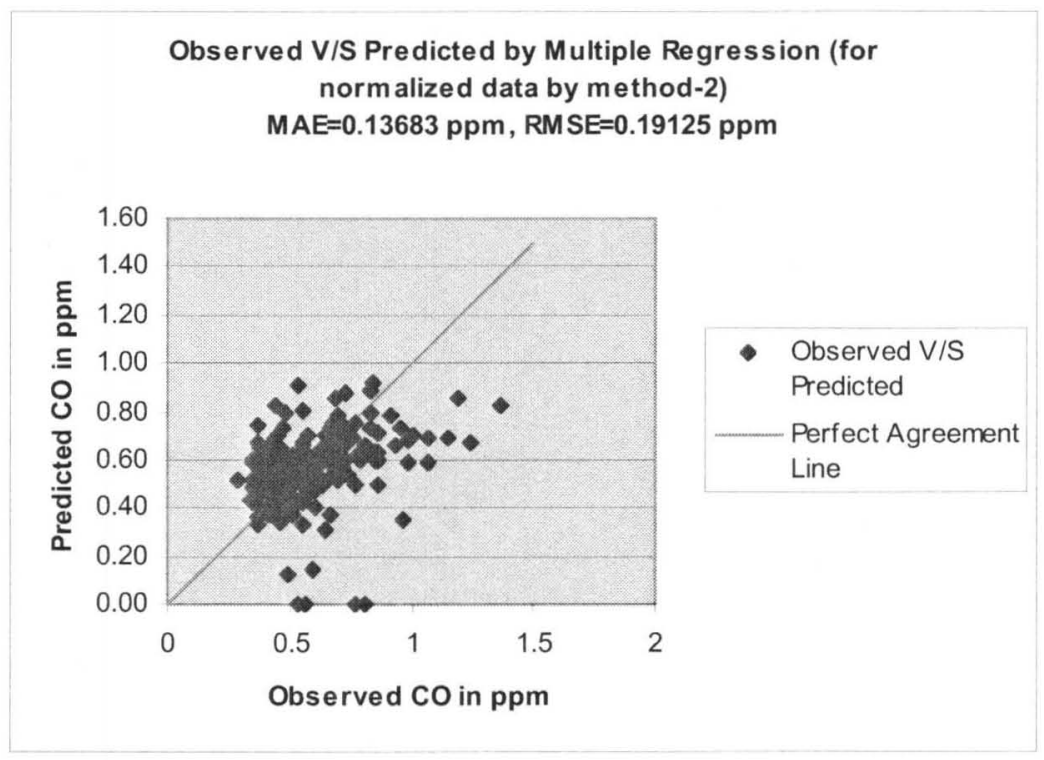

Figure 5.12

Observed versus Predicted values by MR model for normalized data by method-2 


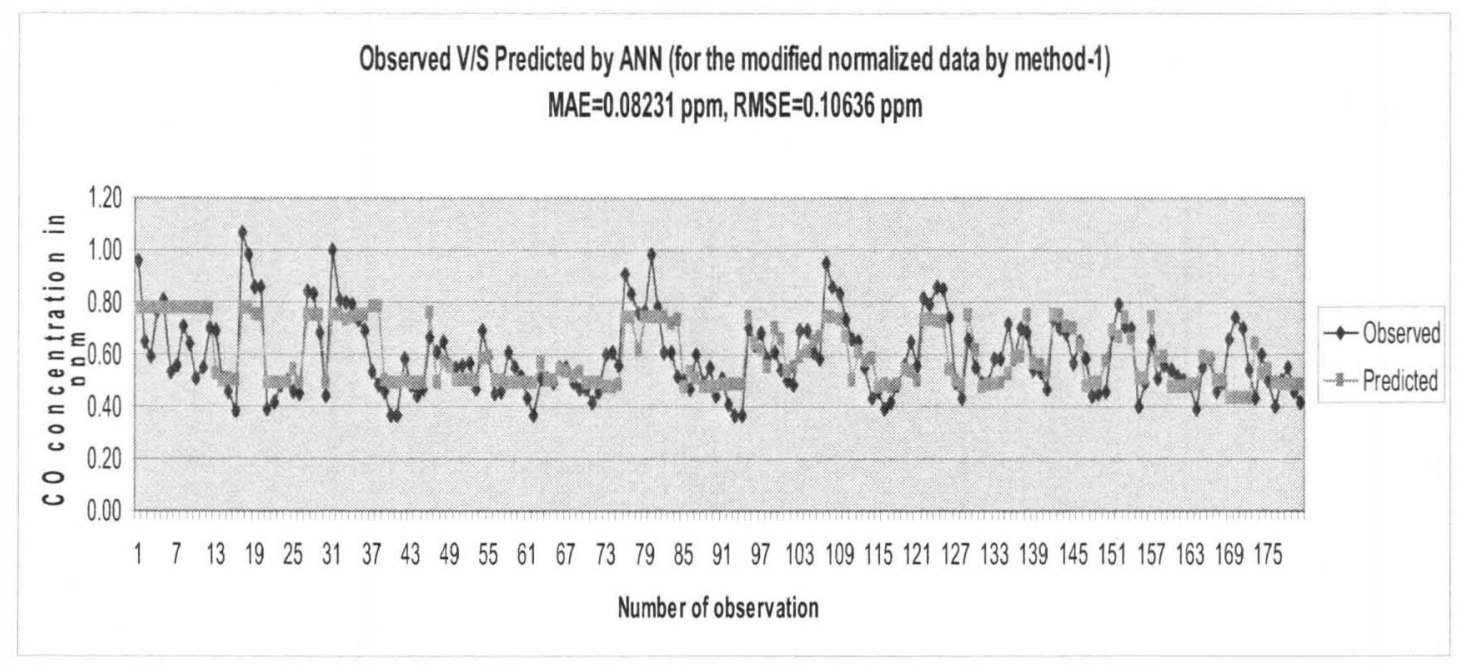

Figure 5.13

Observed versus Predicted values by ANN model for modified normalized data by method-1

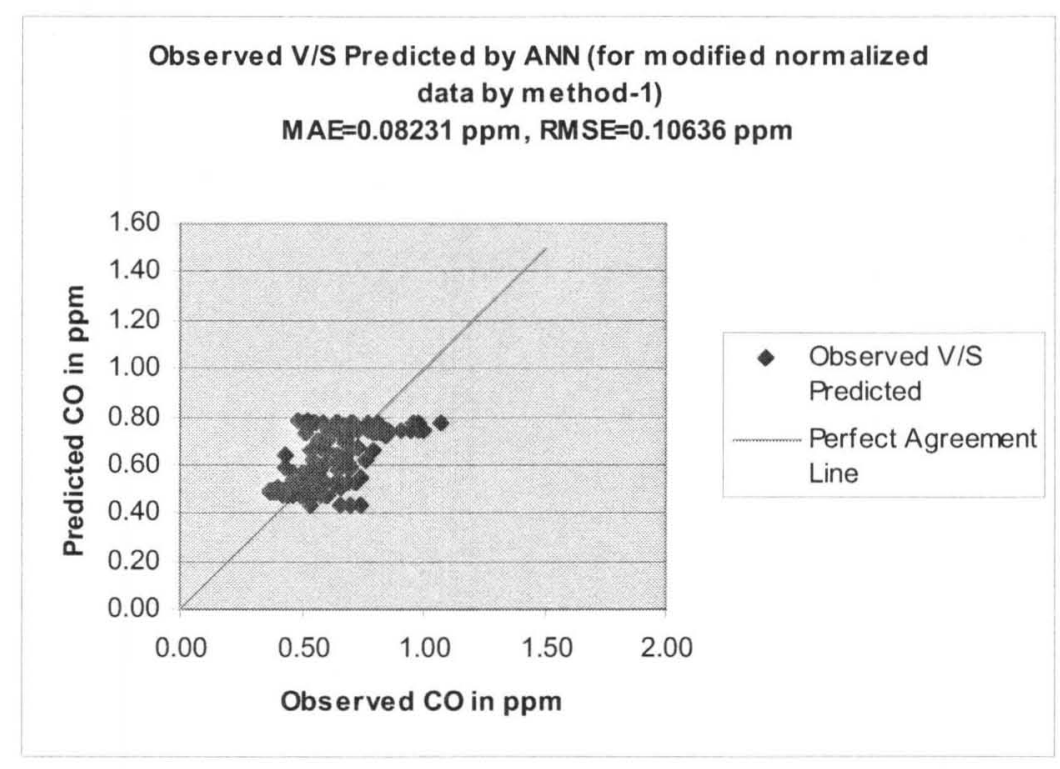

Figure 5.14

Observed versus Predicted values by ANN model for modified normalized data by method-1 


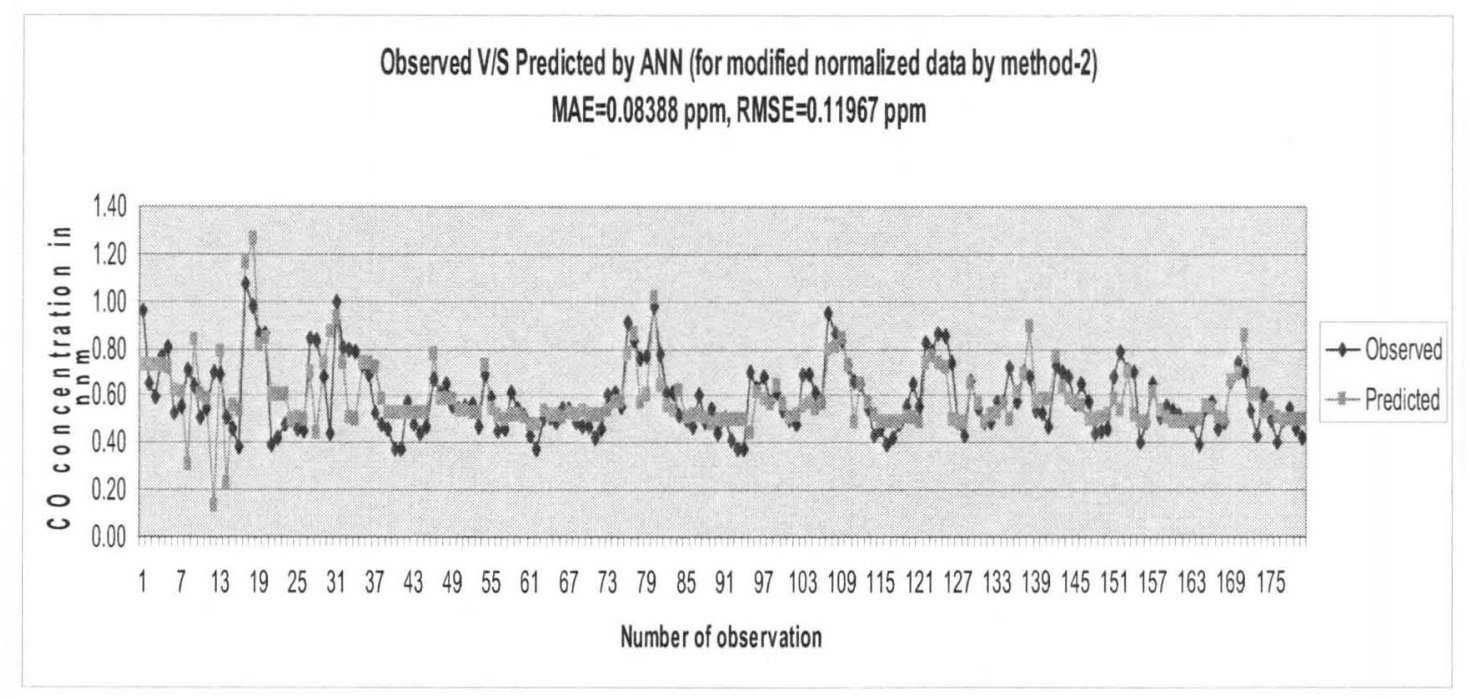

Figure 5.15

Observed versus Predicted values by ANN model for modified normalized

data by method-2

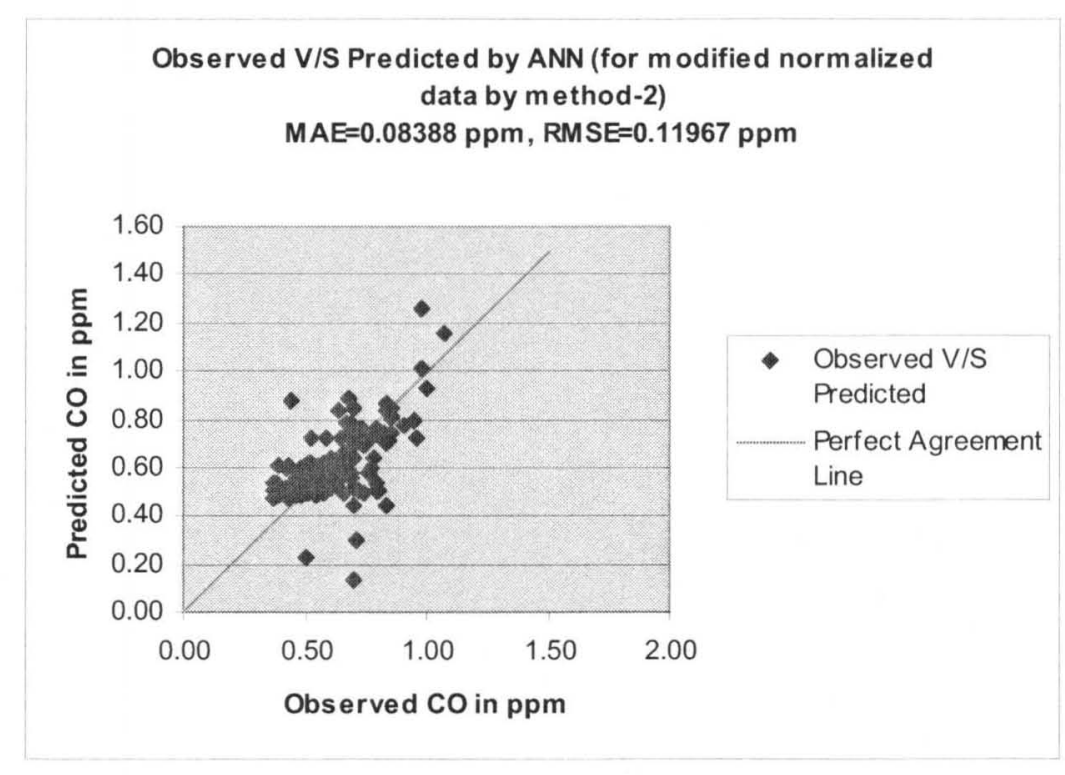

Figure 5.16

Observed versus Predicted values by ANN model for modified normalized data by method-2 


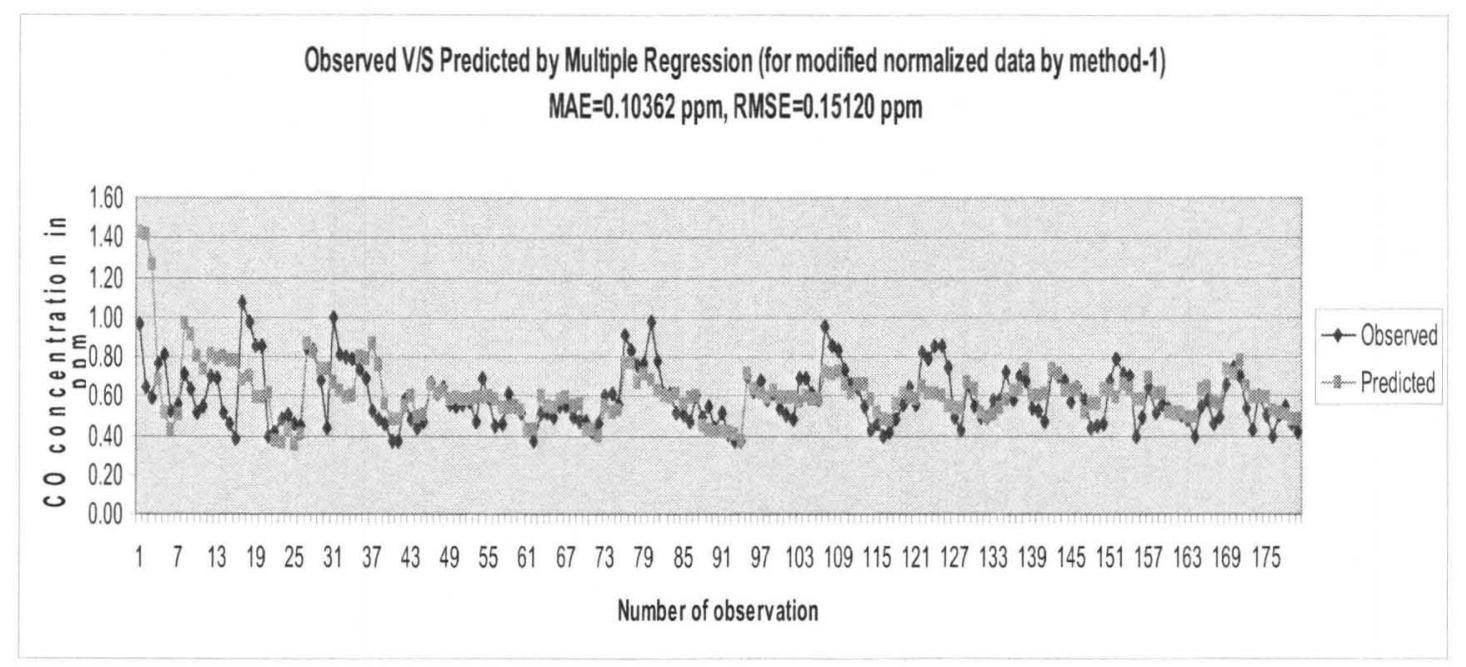

\section{Figure 5.17}

Observed versus Predicted values by MR model for modified normalized data by method-1

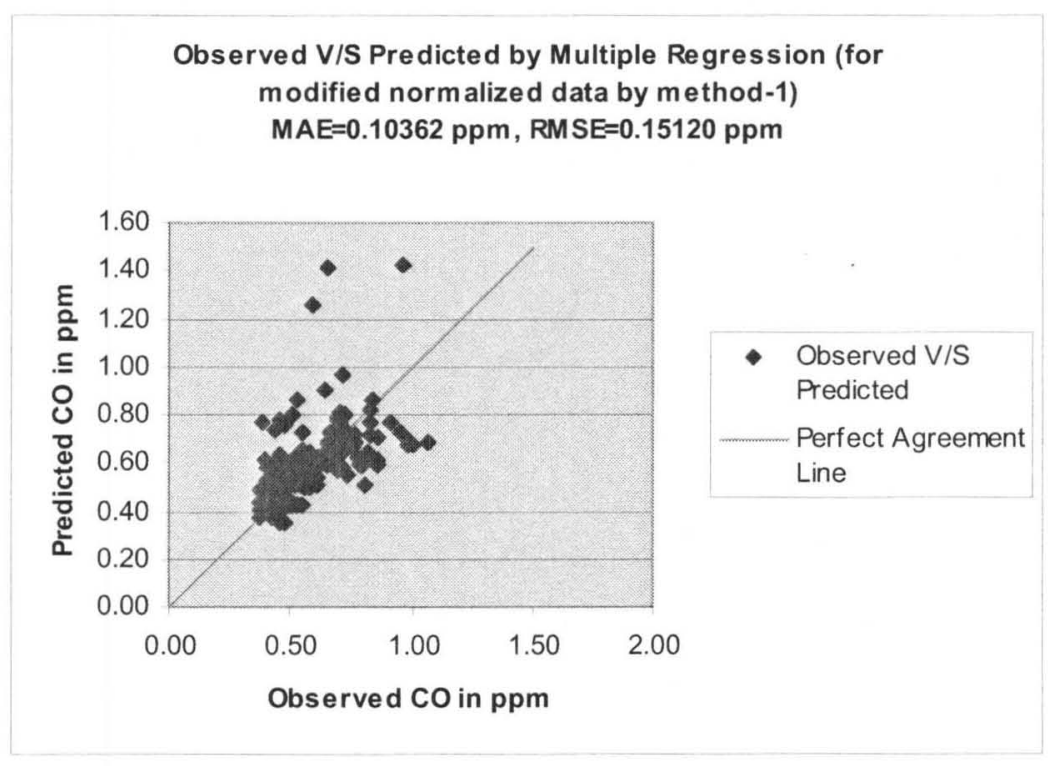

Figure 5.18

Observed versus Predicted values by MR model for modified normalized data by method-1 


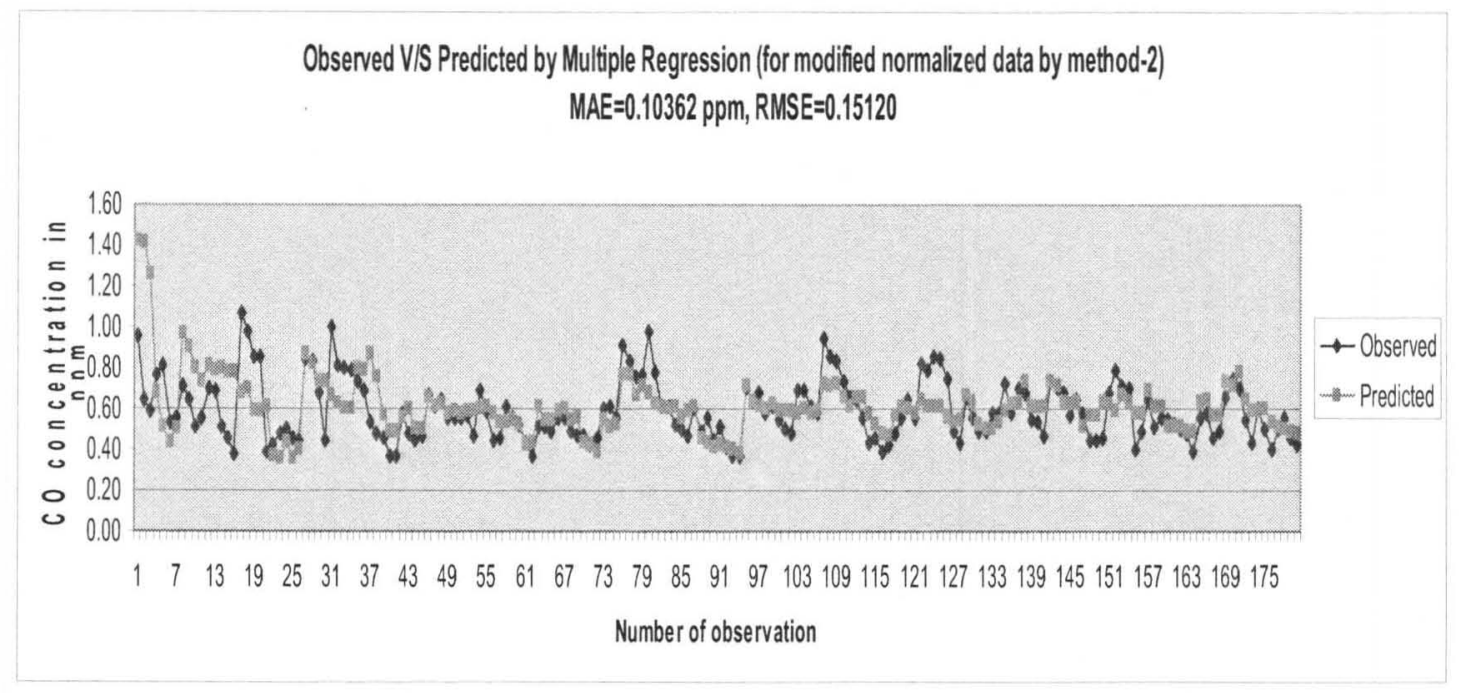

Figure 5.19

Observed versus Predicted values by MR model for modified normalized data

by method-2

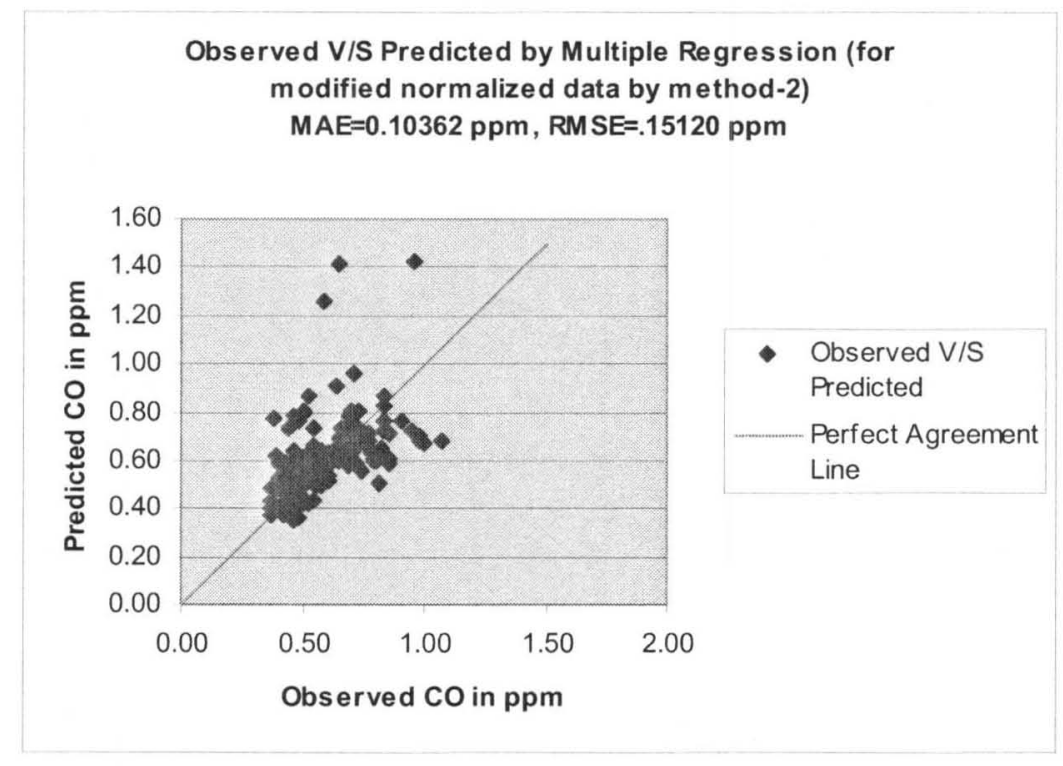

Figure 5.20

Observed versus Predicted values by MR model for modified normalized data by method-2 


\subsubsection{Discussion}

The optimal ANN models (one for each set of data), defined here as those with the best or lowest MAE values, were used to predict the concentrations of $\mathrm{CO}$ in ambient air. The prediction or validation was performed by furnishing the ANN models with only the operationally available values of observation variables. With regard to the MR models too, the same set of input values were used so that a valid comparison between the two model types (ANN and MR) can be evaluated. The following section describes the notable observations derived from the $\mathrm{CO}$ modeling results.

- The values for MAEs and RMSEs are lower (better) for modified data as compared with use of the original data for both the model types-ANN and MR models- and it strengthens the concept that the removal of outlicrs improves model performance irrespective of modeling approach. Prediction with modified data was consistently better and more reliable.

- The graphical comparison of predicted and observed CO concentration closely follow one another in the case of both ANN and MR models, indicating the methods are well suited to the application and the approach can yield potentially useful results for research or planning applications. .

- Using quantitative measures of performance, the differences between predicted and observed $\mathrm{CO}$ values are lower in the case of ANN modeling, and this indicates an improved modeling approach relative to MR.

- The performance criteria values for both MAE and RMSE are less in the case of ANN models indicating both robustness and accuracy of ANN over MR models. 
- Scatter plots support the same fact - the ANN's supremacy over MR. Considering the data spread around the 1:1 line or "Perfect Agreement Line" that is less in the case of ANN, supporting the conclusion of superior model performance. This is also indicated by the lower values of RMSE in the case of ANN as compared with the MR model.

- The distribution in number of data points about the "Perfect Agreement Line" is equally distributed (above and below) in all the scatter plots indicating unbiased ANN model performance.

- The distribution of data point predictions shown in both the scatter plots and point comparisons of predicted and observed values are identical for the MR models using either method of data normalization. This result shows the data normalization method does not affect MR model and this fact is also supported by the values of MAEs and RMSEs being equal in MR modeling for both methods of data normalization.

- But abovementioned fact is not applicable to ANN modeling as the plots and curves are different and the values of MAEs and RMSEs as well. The method of data normalization therefore does affect the ANN modeling and is a very crucial element to be decided by trial and crror.

\subsubsection{Effect of ITS on ambient air quality}

\subsubsection{Results}

In this section, the effect of ITS on ambient air quality has been highlighted. Using trained network to learn about the effect of ITS on air quality (concentration of $\mathrm{CO}$ ), 
involved running the network with all of the input neurons, except the one of ITS, held constant; interactions could be explored by setting ITS at various values-starting from $0 \%$ through $100 \%$ with an increment of $2 \%$. As it can be seen from Figure 5.21 , five curves have been plotted-four for the four sets of data with ANN and one for the MR model.

\%ITS VIS CO Concentration in ambient air

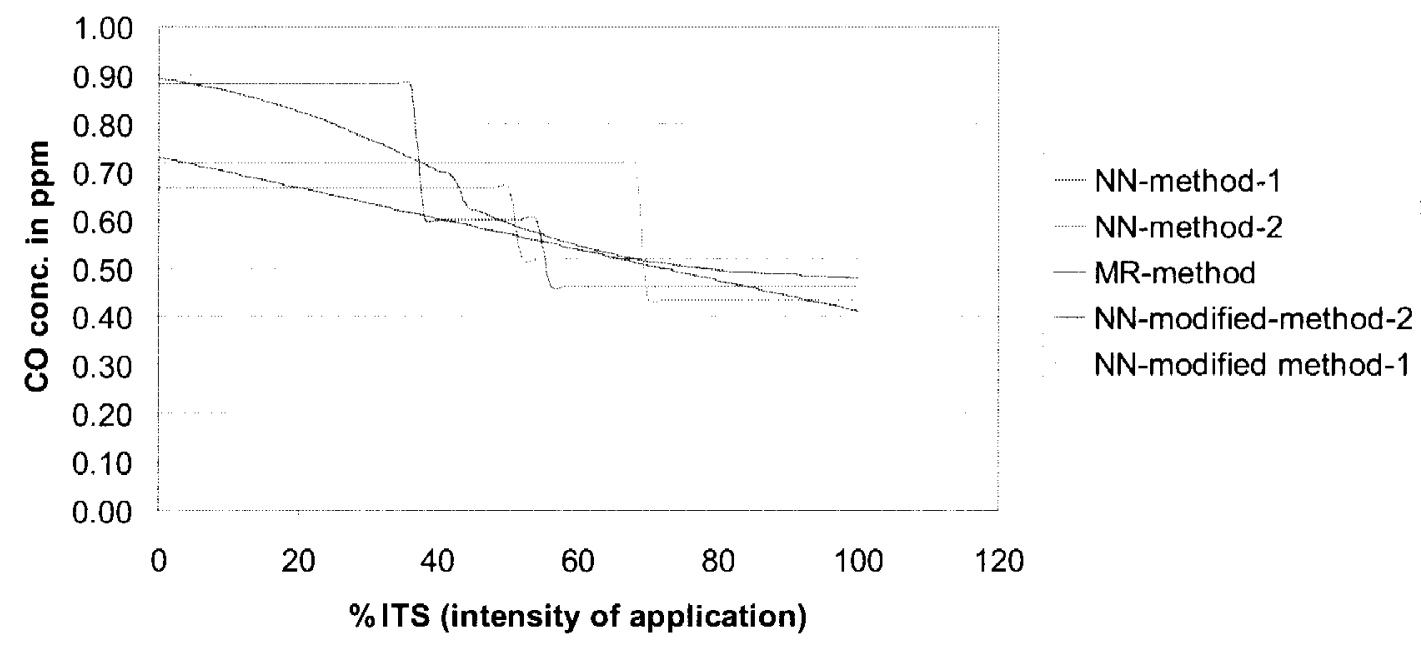

Figure 5.21

$\%$ ITS (intensity of application) versus $\mathrm{CO}$ concentration in ambient air

\subsubsection{Discussion}

Following section describes the important observations about the results of $\mathrm{CO}$ modeling with regard to ITS effects on air quality:

- As it can be seen from Figure 5.21, CO pollution in ambient air decreases as ITS application intensity increases irrespective of the method of data normalization and the data used (original or modified). In both the 
models-ANN and MR, it shows that ITS helps in curbing down the CO pollution.

- Induce demand increases pollution because more number of vehicles would be on the road due to smoothening of traffic by ITS.

- Since the average speed on roads is increased because of less delay or congestion due to ITS, the rate of emission also increases that causes more pollution.

- In contrast, because of less congestion or delay, fuel saving takes place causing significant reduction in emissions of motor vehicles. This contributes in reduction of CO pollution in ambient air.

- Since overall ITS helps in curbing the CO pollution in ambient air as it evident from Figure 5.21, reason that one could think of might be, "the cumulative effect of induced demand, effect of increase of average speed on emission rates of $\mathrm{CO}$ and reduction of congestion/delay results in the reduction of CO concentration". 


\subsection{Nitrogen Dioxide $\left(\mathrm{NO}_{2}\right)$}

Several configurations of ANN model structure (combinations of nodes and hidden layers) of ANN were implemented and applied using sets of the $\mathrm{NO}_{2}$ data- four sets altogether. These sets, like $\mathrm{CO}$ data sets in previous section, were as follows:

Set 1: Original data normalized by method-1 (n1),

Set 2: Modified data normalized by method-1 (nl),

Set 3: Original data normalized by method-2 (n2) and,

Set 4: Modified data normalized by method-2 (n2).

The notations $\mathrm{n} 1$ and $\mathrm{n} 2$ indicate the two methods of data normalization as described in Chapter-IV.

\subsubsection{ANN Architecture Selection}

\subsubsection{Results}

In this section, the influence of ANN architecture on the MAE performance measures has been highlighted with regard to $\mathrm{NO}_{2}$ modeling. Starting with very simple architecture of ANN and subsequently increasing the model structure complexity, MAE performance measures were determined corresponding to each model configuration or architecture. Also, a Multiple Regression study was performed on each of the four data sets and the corresponding MAE values were determined. Tables 5.5 through 5.8 summarize the model architecture configurations and corresponding values of MAE for cach and Figure 5.22 through 5.25 depict the corresponding plots. Notations in this section are same as in the previous section and the same is the description about the figures. Only difference is that this section deals with $\mathrm{NO}_{2}$. 
Table 5.5 ANN model performance according to architecture and corresponding MAE measure (for data normalized by method-1)

Where:

\begin{tabular}{|c|c|}
\hline Configuration-n1 & \%E (MAE) \\
\hline$(1-1-0)^{\star}$ & 34.8 \\
\hline$(1-2-0)$ & 27.8 \\
\hline$(1-3-0)$ & 34.1 \\
\hline$(1-4-0)$ & 29.3 \\
\hline$(1-5-0)$ & 24.9 \\
\hline$(1-6-0)$ & 27.8 \\
\hline$(2-1-1)$ & 34.7 \\
\hline$(2-2-2)$ & 33.4 \\
\hline$(2-3-3)$ & 29.0 \\
\hline$(2-4-4)$ & 24.2 \\
\hline$(2-5-5)$ & 33.6 \\
\hline Multiple Regression & 35.2 \\
\hline
\end{tabular}

Meanings of various terms and notations are same as mentioned in the previous sections.

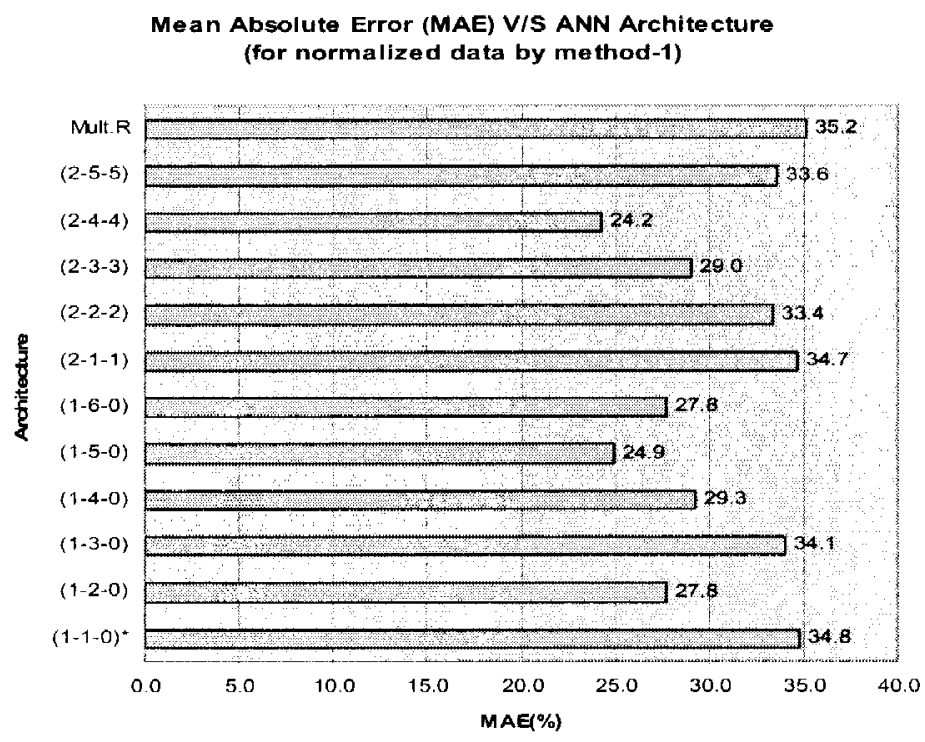

Figure 5.22

ANN performance summary according to architecture and corresponding

MAE (for normalized data by method-1) 
Table 5.6 ANN model performance according to architecture and corresponding MAE measure (for modified normalized data by method-1)

\begin{tabular}{|c|c|}
\hline Configuration-n1-m & $\% \mathrm{E}$ (MAE) \\
\hline$(1-1-0)$ & 22.8 \\
\hline$(1-2-0)$ & 29.9 \\
\hline$(1-3-0)$ & 35.5 \\
\hline$(1-4-0)$ & 33.7 \\
\hline$(1-5-0)$ & 25.0 \\
\hline$(2-1-1)$ & 25.4 \\
\hline$(2-2-2)$ & 26.7 \\
\hline$(2-3-3)$ & 38.3 \\
\hline$(2-4-4)$ & 24.2 \\
\hline$(2-5-5)$ & 19.3 \\
\hline$(2-6-6)$ & 27.8 \\
\hline$(2-2-1)$ & 24.8 \\
\hline$(2-1-2)$ & 27.7 \\
\hline$(2-3-4)$ & 25.9 \\
\hline$(2-5-6)$ & 27.1 \\
\hline Multiple Regression & 30.3 \\
\hline
\end{tabular}

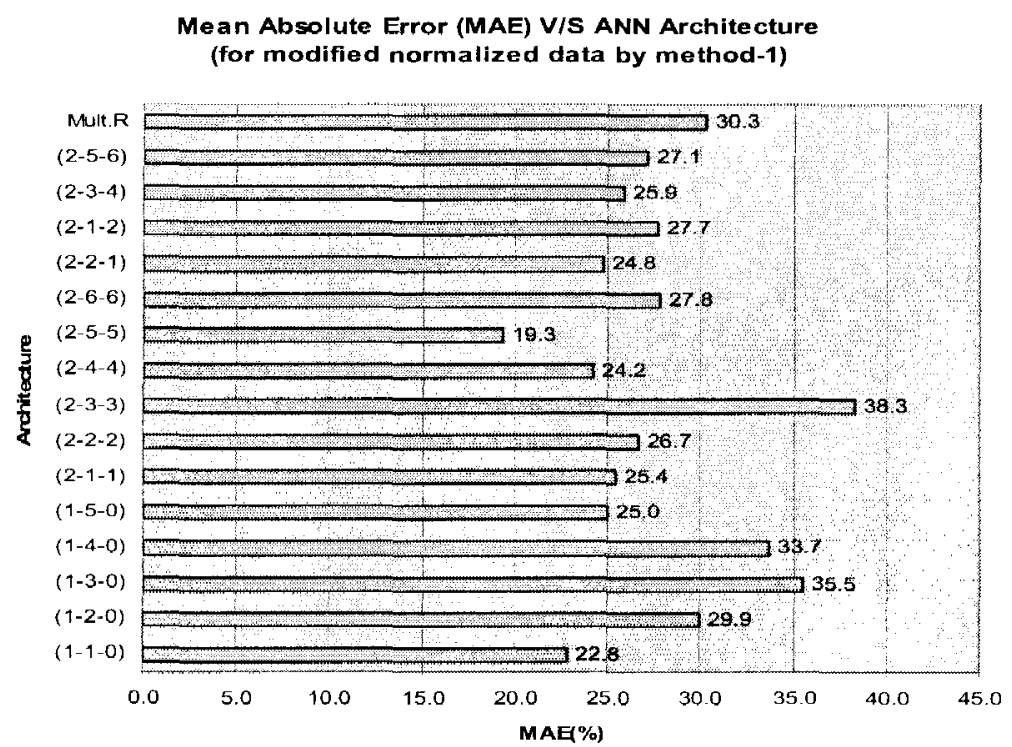

Figure 5.23

ANN performance summary according to architecture and corresponding

MAE (for modified normalized data by method-1) 
Table 5.7 ANN model performance according to architecture and corresponding MAE measure (for normalized data by method-2)

\begin{tabular}{|c|c|}
\hline Configuration-n2 & $\% \mathrm{E}$ (MAE) \\
\hline$(1-1-0)$ & 34.4 \\
\hline$(1-2-0)$ & 31.0 \\
\hline$(1-3-0)$ & 33.7 \\
\hline$(1-4-0)$ & 32.3 \\
\hline$(2-1-1)$ & 37.7 \\
\hline$(2-2-2)$ & 32.0 \\
\hline$(2-3-3)$ & 34.1 \\
\hline$(2-4-4)$ & 31.0 \\
\hline$(2-5-5)$ & $\mathbf{2 5 . 5}$ \\
\hline$(2-6-6)$ & 36.5 \\
\hline Multiple Regression & 35.2 \\
\hline
\end{tabular}

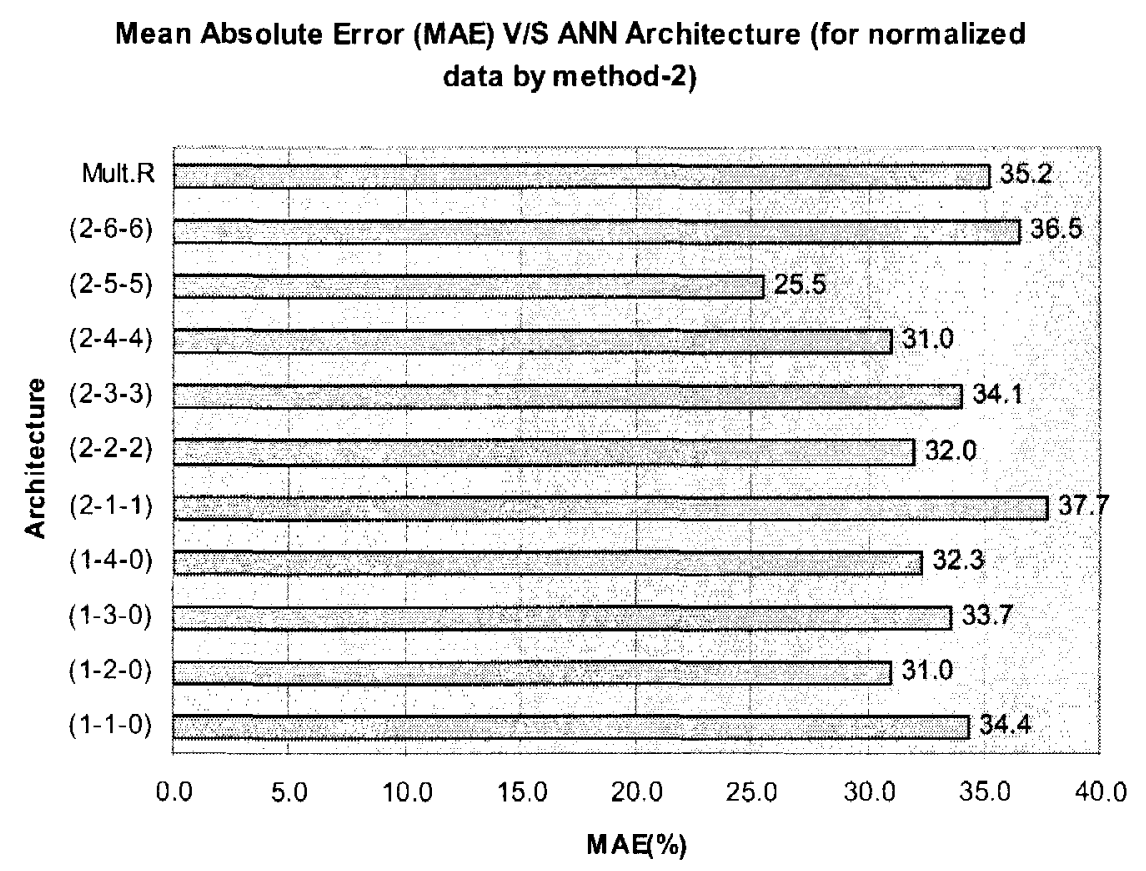

Figure 5.24

ANN performance summary according to architecture and corresponding MAE (for normalized data by method-2) 
Table 5.8 ANN model performance according to architecture and corresponding MAE measure (for modified normalized data by method-2)

\begin{tabular}{|c|c|}
\hline Configuration-n2-m & $\% \mathrm{E}$ \\
\hline$(1-1-0)$ & 22.9 \\
\hline$(1-2-0)$ & 27.4 \\
\hline$(1-3-0)$ & 28.0 \\
\hline$(1-4-0)$ & 29.7 \\
\hline$(1-5-0)$ & 26.2 \\
\hline$(2-1-1)$ & 24.5 \\
\hline$(2-2-2)$ & 29.0 \\
\hline$(2-3-3)$ & $\mathbf{2 2 . 0}$ \\
\hline$(2-4-4)$ & 36.5 \\
\hline$(2-4-3)$ & 33.0 \\
\hline Mult.R & 30.1 \\
\hline
\end{tabular}

\section{Mean Absolute Error (MAE) V/S ANN Architecture (for modified normalized data by method-2)}

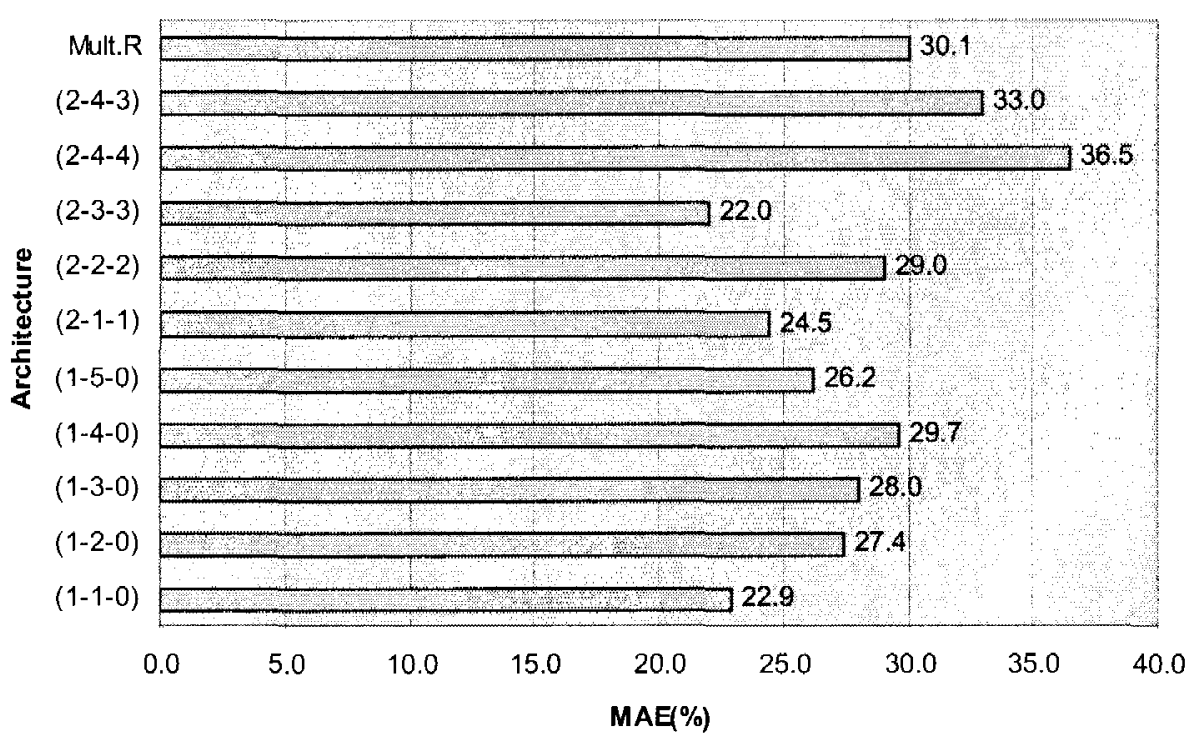

Figure 5.25

ANN performance summary according to architecture and corresponding

MAE (for modified normalized data by method-2) 


\subsubsection{Discussion}

Like CO modeling mentioned in previous sections, several ANN model configurations or architectures were applied with $\mathrm{NO}_{2}$ data in order to arrive at the optimal form to minimize MAE. MAE is a standard performance measure to check the capability of a prediction model.

Entries in bold in the tables indicate the configuration of ANN with the lowest MAE in prediction. Following section summarizes the significant observations about the results of $\mathrm{NO}_{2}$ modeling with regard to variations in ANN Architecture.

- The values of MAE for Set 1 data varied from $24.2 \%$ corresponding to ANN model configuration (2-4-4) to $34.8 \%$ corresponding to ANN model configuration (1-1-0), and 35.2\% for the Multiple Regression model.

- The MAE values for Set 2 data varied from $19.3 \%$ corresponding to ANN model configuration (2-5-5) to $38.3 \%$ corresponding to ANN model configuration (2-3-3) and 30.1\% for Multiple Regression model.

- The values of MAE for Set 3 data varied from $25.5 \%$ corresponding to ANN model configuration (2-5-5) to $37.7 \%$ corresponding to ANN model configuration (2-1-1) and 35.2\% for Multiple Regression model.

- The values of MAE for Set 4 data varied from $22.0 \%$ corresponding to ANN model configuration (2-3-3) to $36.5 \%$ corresponding to ANN model configuration (2-4-4) and 30.1\% for Multiple Regression model.

- The values of MAEs with Multiple Regression are same for Set 1 \& Set 3 $(35.2 \%)$ and Set-2 \& Set-4 (30.1\%) indicating that the influence of the 
data normalization method does not affect the multiple regression modeling results.

- From Tables 5.5 through 5.8, it is evident that MAEs are larger when the ANN model is either under-trained or over-trained; however there is no clear-cut rule by which one can define the optimal ANN structure. Although the tendency is for more complex the relationships between dependent and independent variables require more complex ANN structures, the final details of model structure must still be determined using an iterative, trial and error style procedure. Through the training or model calibration process one must monitor the ANN evolution and avoid both under-trained and over-trained regions of the solution space.

- The values MAEs in prediction application for $\mathrm{NO}_{2}$ vary over the range from $19.3 \%$ to $25.5 \%$ and those are justifiable considering the fact that the significant $\mathrm{NO}_{2}$ emissions are from sources, in addition to the transportation factors, include industries, power plants etc. and those factors have not been included in this study.

- The lowest MAE for Set 1 data was $24.2 \%$ and $19.3 \%$ for Set 2 (modified data by removing outliers) indicating that the by removing "outliers" the prediction error can be reduced significantly. This same result was found for the data Set 3 and 4 data and also for the Multiple Regression modeling. Removal of outliers therefore always improves the accuracy of any prediction model. 
- The Lowest value of MAE for data Set 1 (normalized by method-1, n1) was $24.2 \%$ and was $25.5 \%$ for Set 3 (normalized by method-2, n2). Similarly, lowest MAE for data Set 2 (normalized by method-1, n1) was $19.3 \%$ and was $22.0 \%$ for Set 4 (normalized by method-2, n2). This result indicates that the method of normalization does significantly affect the results of ANN modeling and in this particular study, method number one (nl) proved to be providing more accurate prediction relative to method number two (n2).

\subsubsection{Comparison of Observed and Predicted values}

\subsubsection{Results}

The observed and predicted results from both the models-ANN model and Multiple Regression (MR) model-were compared using values for both the MAE and RMSE (for the $\mathrm{NO}_{2}$ parameter). The model configuration or architecture providing the least MAE result was selected as the "target" or optimal model and served to provide the predicted values for model validation evaluation. By using 10 -fold technique, the predicted values were found for both the models, the optimal ANN and multiple regression. To provide a means for visualization of the results, a series of curves were plotted comparing observed and predicted values of $\mathrm{NO}_{2}$ concentration. Additionally, a series of scatter plots were developed to illustrate the statistical comparison relative to the perfect agreement line and provide an indication of model bias if any. Figures 5.26 through 5.33 depict the plots of observed and predicted for original data and Figures 5.34 through 5.41 show the plots between observed and predicted for modified data. 


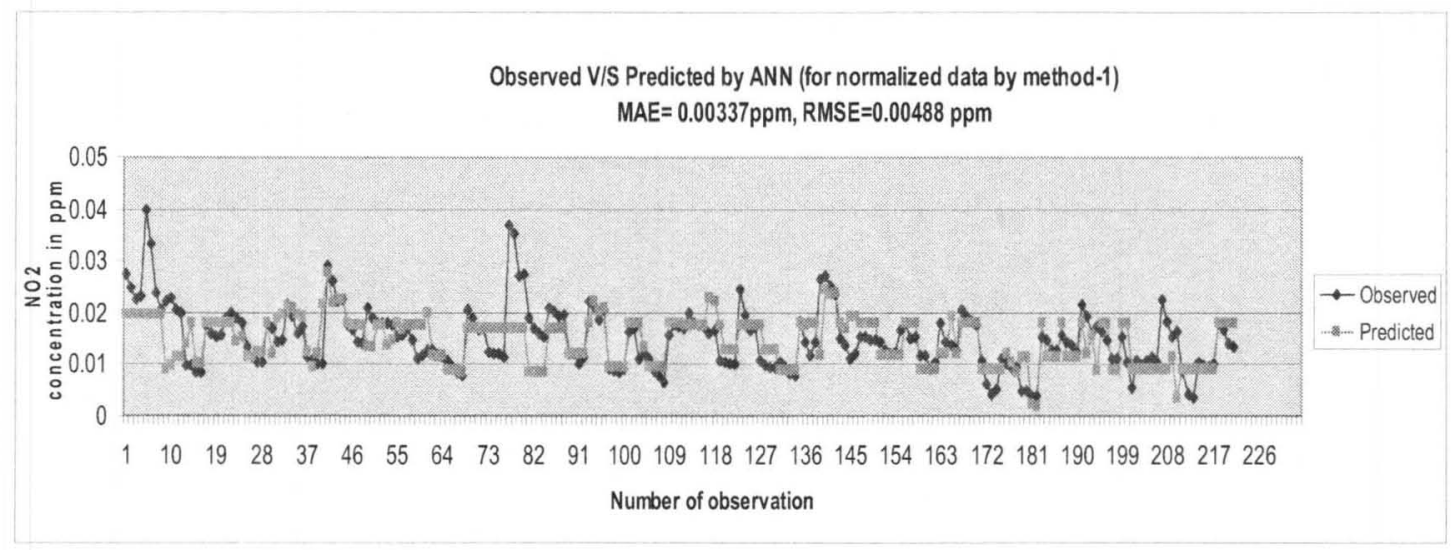

Figure 5.26

Observed versus Predicted values by ANN for normalized data by method-1

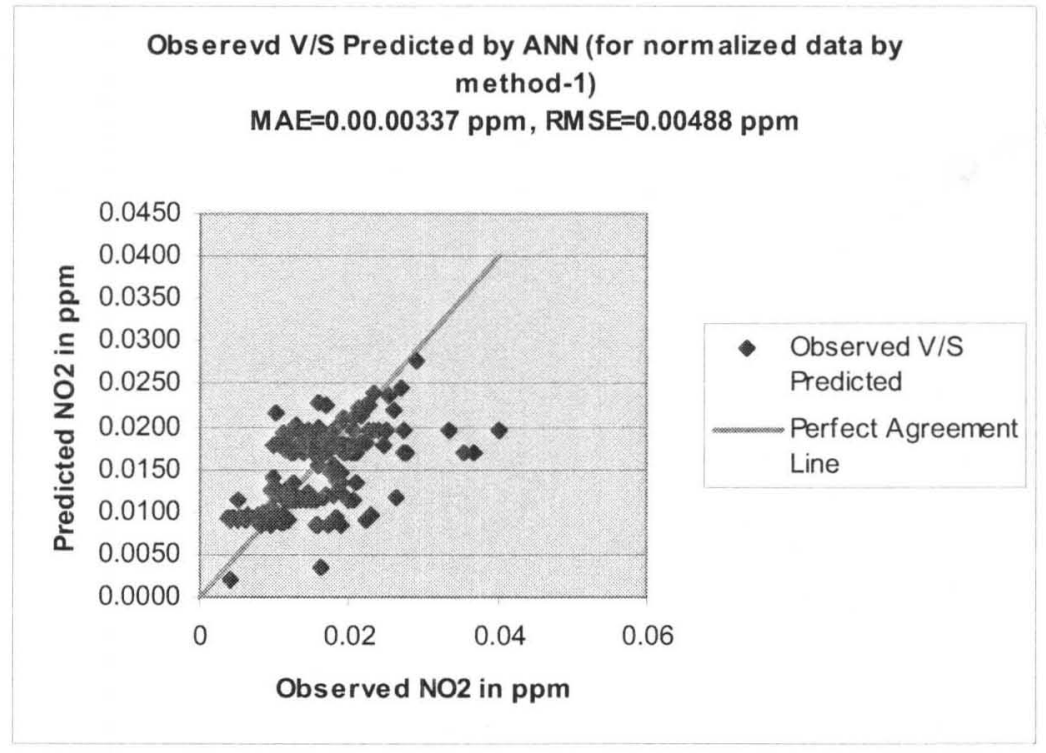

Figure 5.27

Observed and Predicted values by ANN for normalized data by method-1 


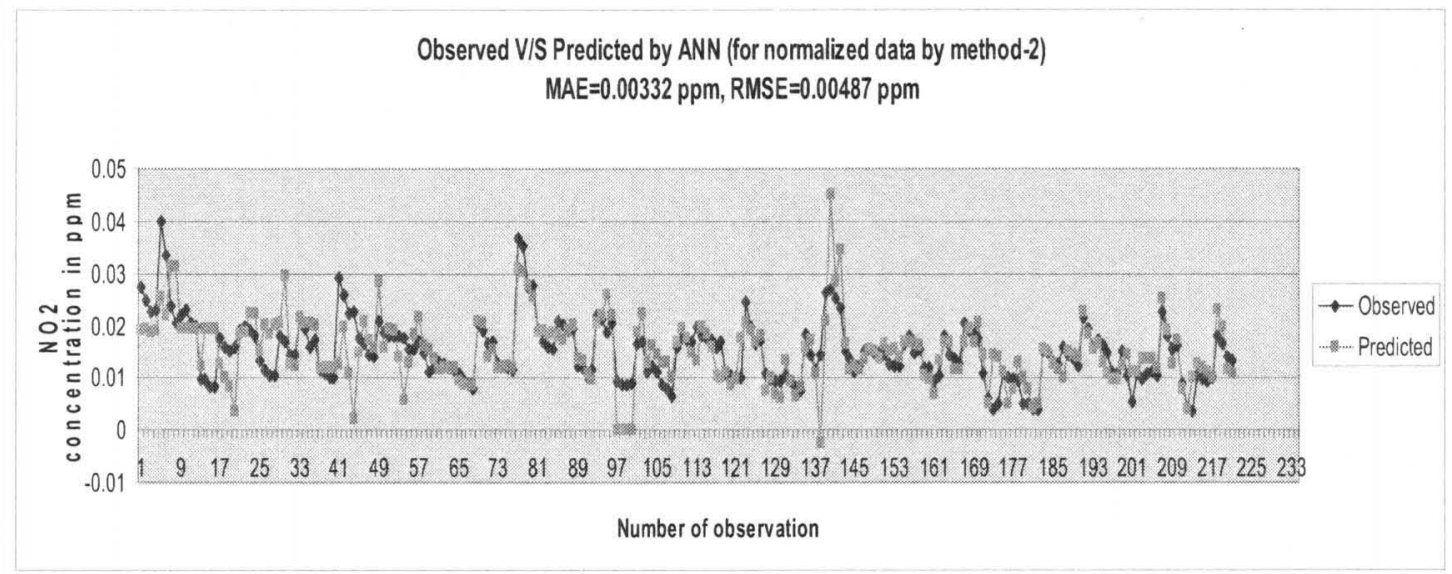

Figure 5.28

Observed versus Predicted values by ANN for normalized data by method-2

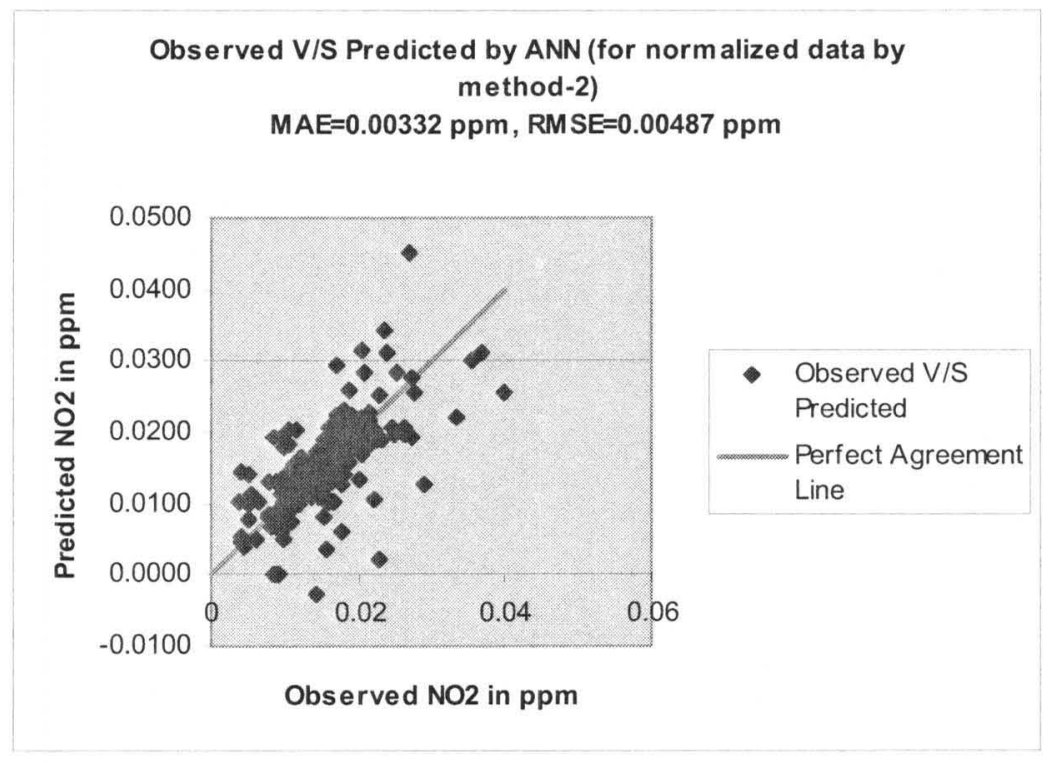

Figure 5.29

Observed and Predicted values by ANN for normalized data by method-2 


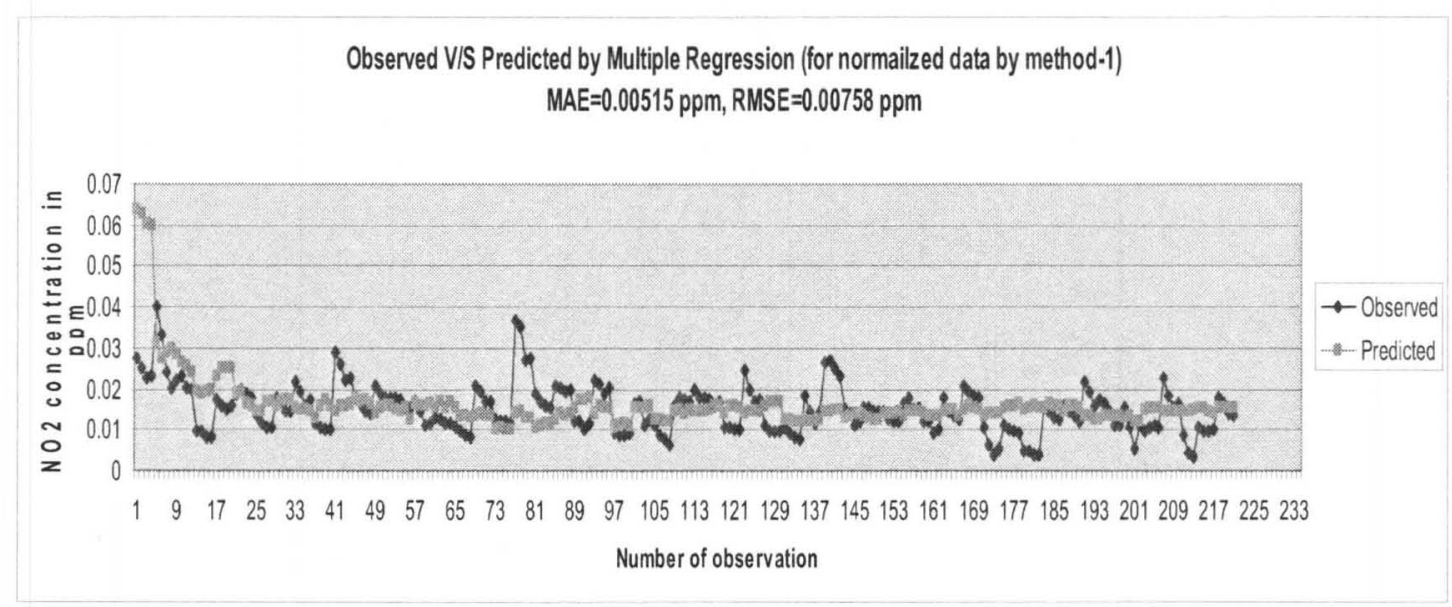

Figure 5.30

Observed versus Predicted values by MR model for normalized data by method-1

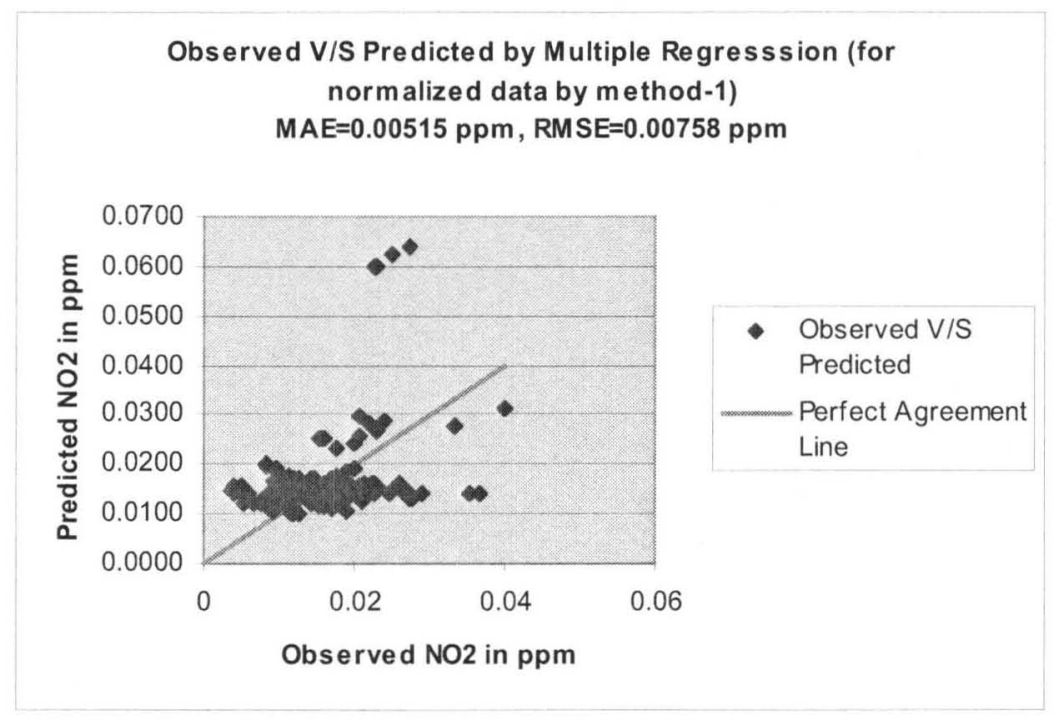

Figure 5.31

Observed and Predicted values by MR model for normalized data by method-1 


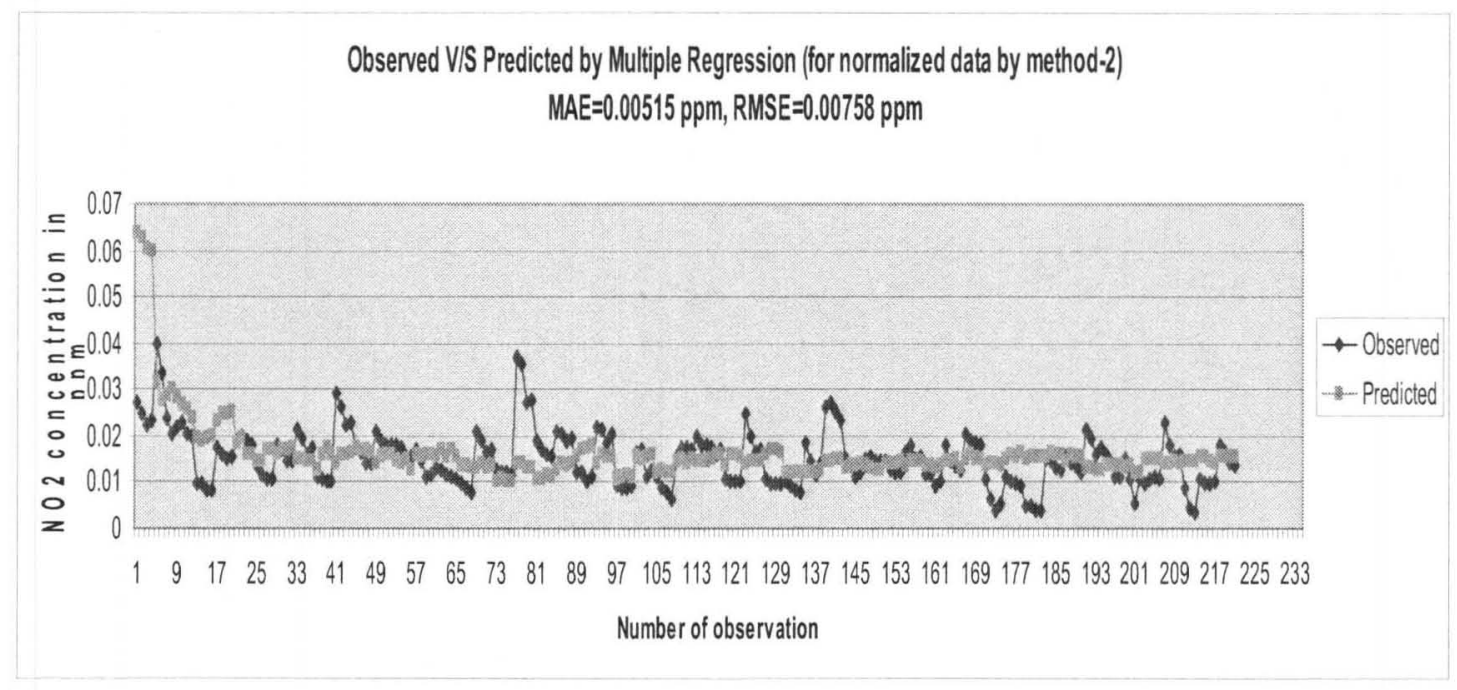

Figure 5.32

Observed versus Predicted values by MR model for normalized data by method-2

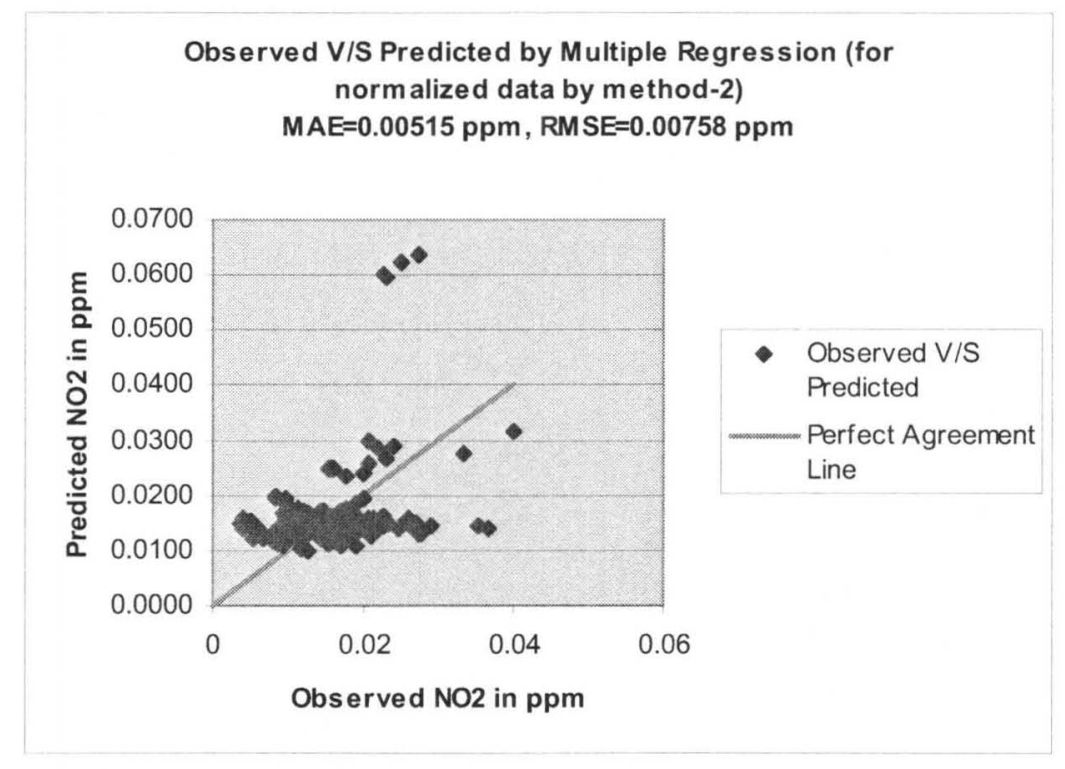

Figure 5.33

Observed and Predicted values by MR model for normalized data by method-2 


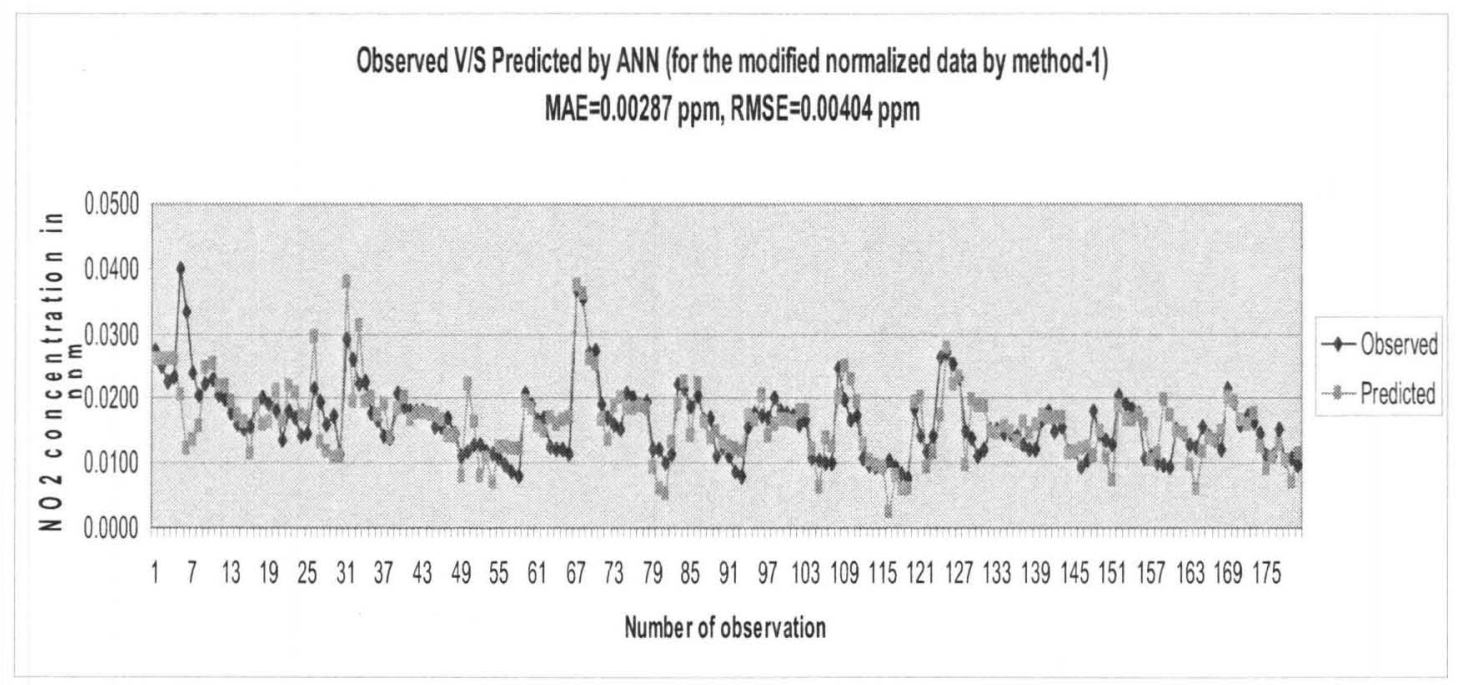

Figure 5.34

Observed versus Predicted values by ANN model for modified normalized data by method-1

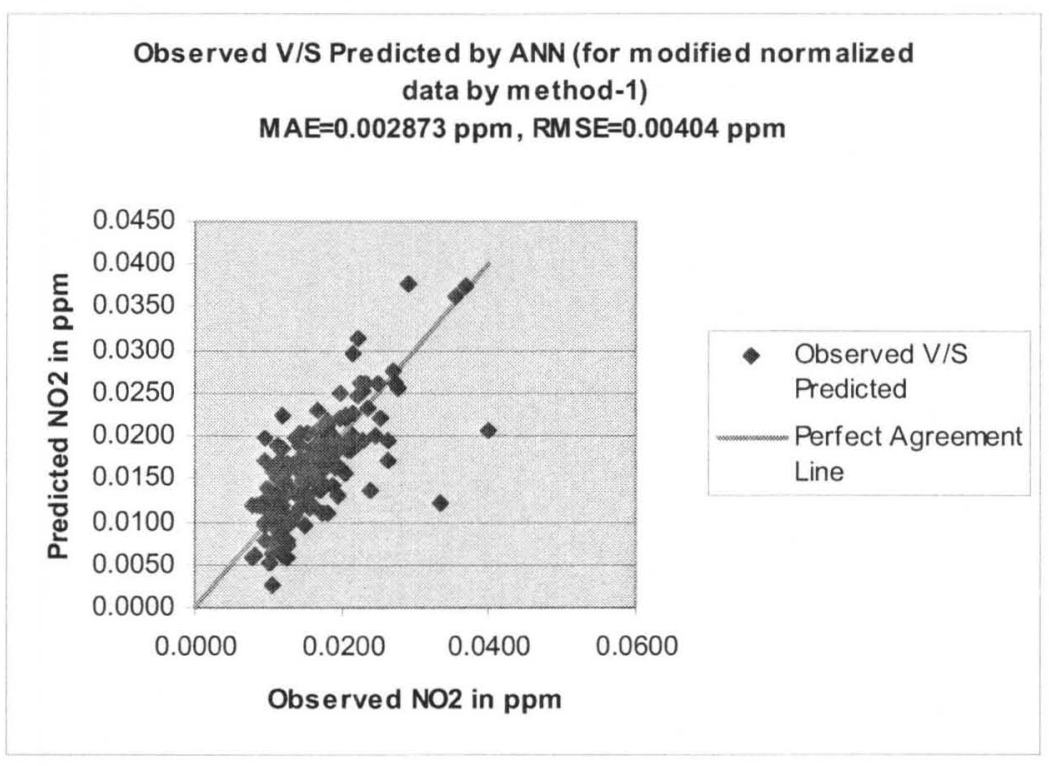

Figure 5.35

Observed and Predicted values by ANN model for modified normalized data by method-1 
Observed VIS Predicted by ANN (for modified normalized data by method-2)

MAE $=0.00337 \mathrm{ppm}, \mathrm{RMSE}=0.00497 \mathrm{ppm}$

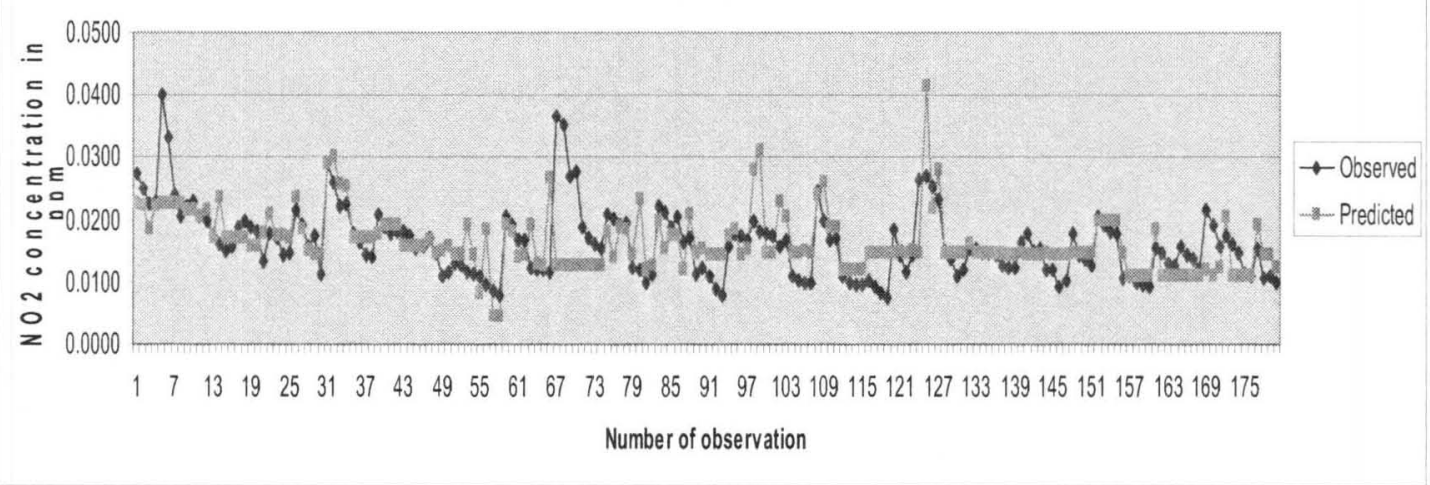

Figure 5.36

Observed versus Predicted values by ANN model for modified normalized

data by method-2

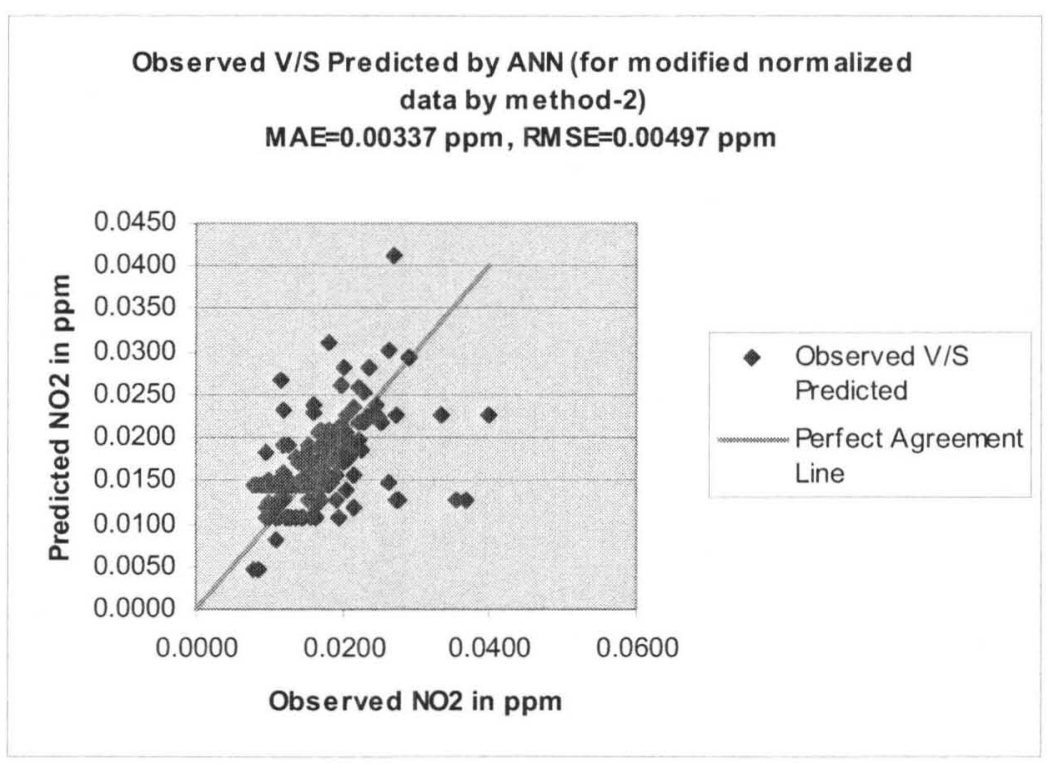

Figure 5.37

Observed and Predicted values by ANN model for modified normalized data

by method-2 


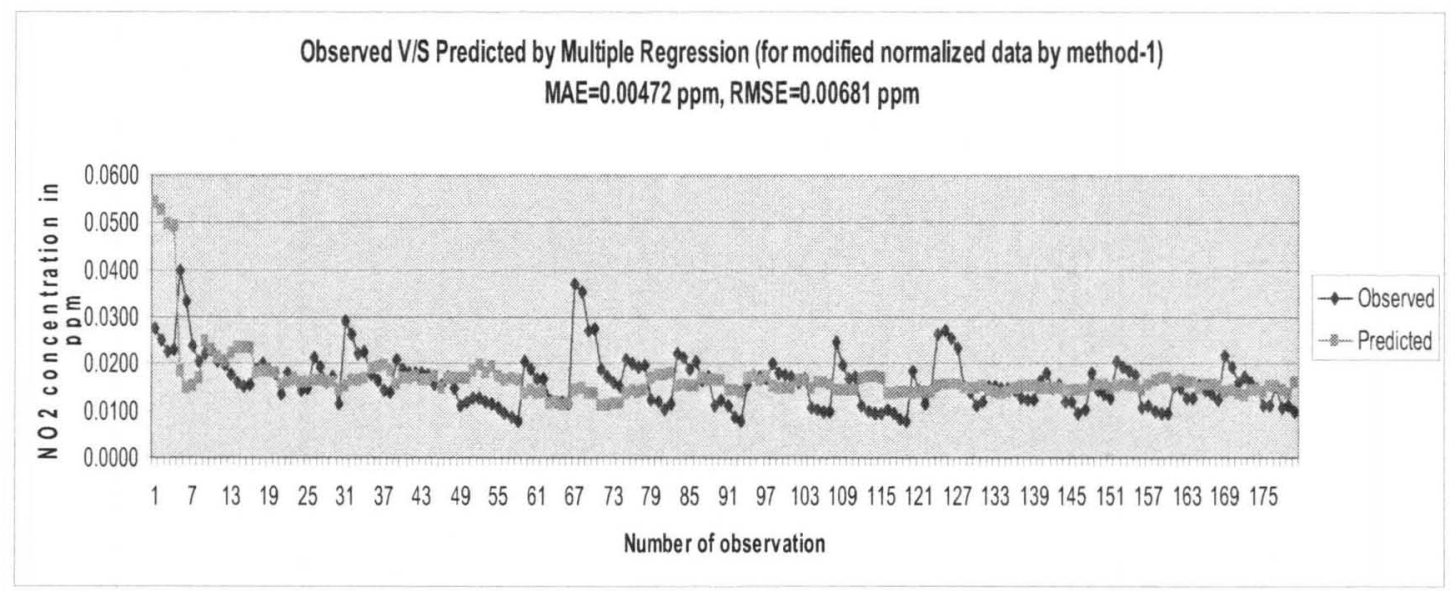

Figure 5.38

Observed versus Predicted values by MR model for modified normalized data by method-1

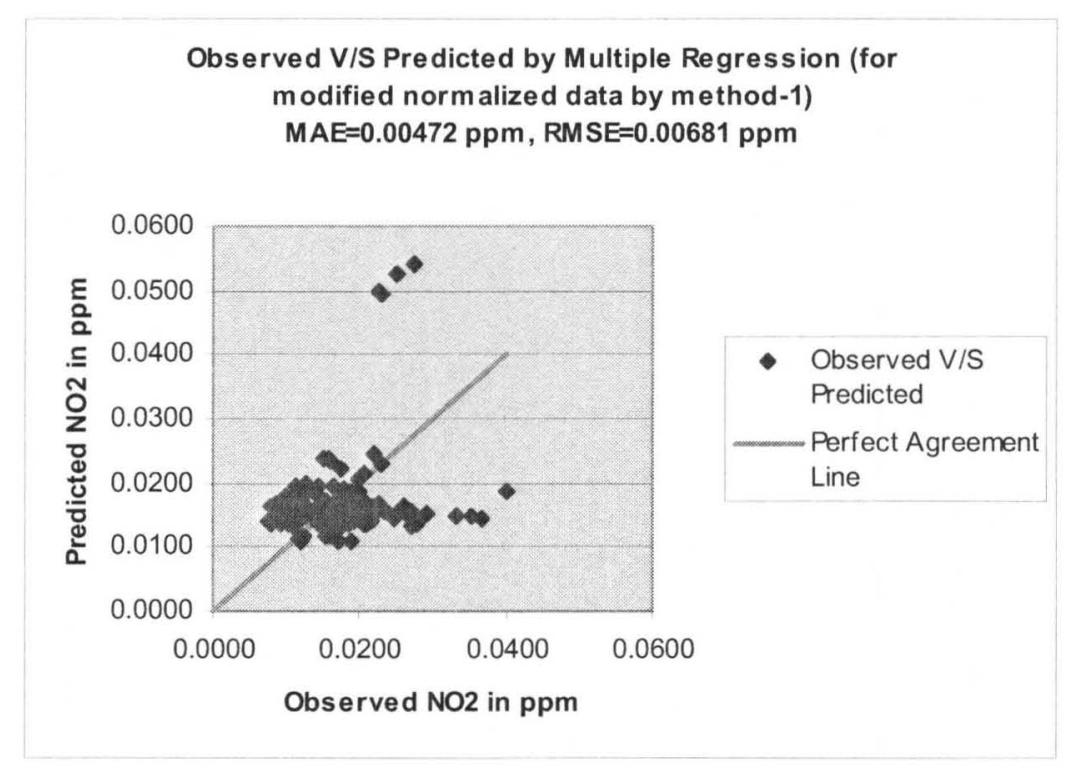

Figure 5.39

Observed and Predicted values by MR model for modified normalized data by method-1 


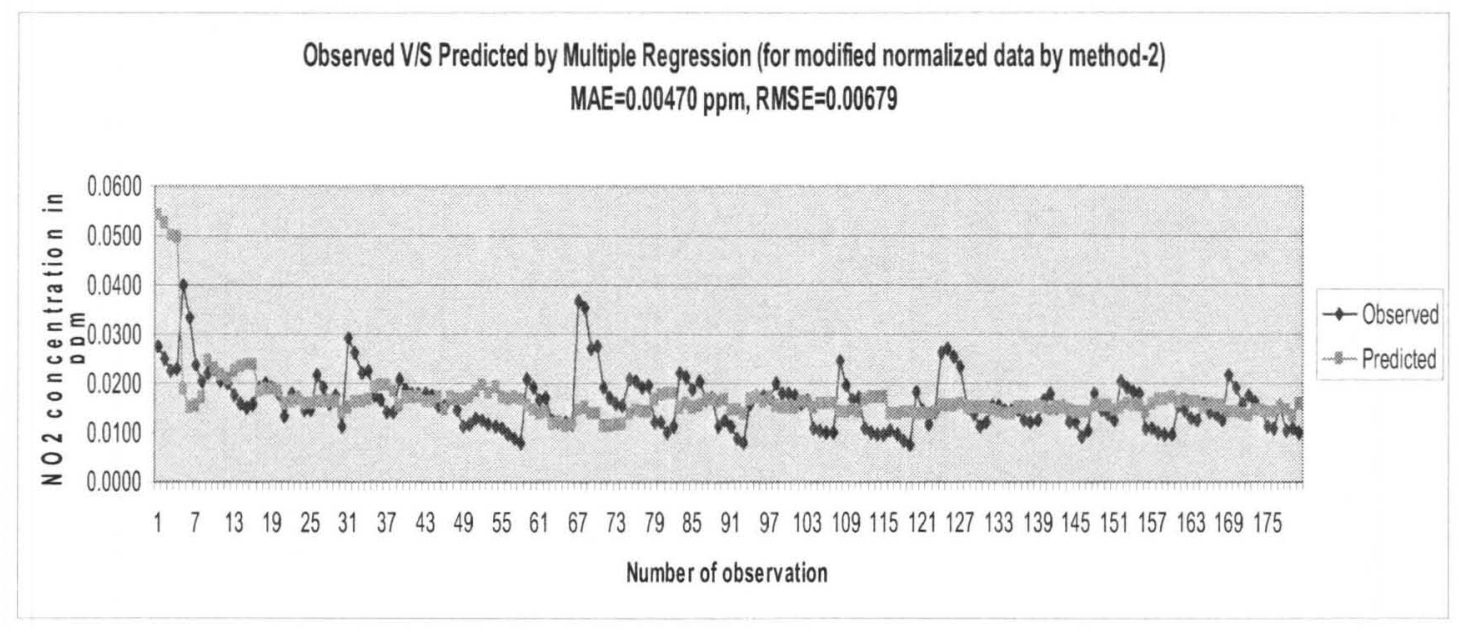

Figure 5.40

Observed versus Predicted values by MR model for modified normalized data by method-2

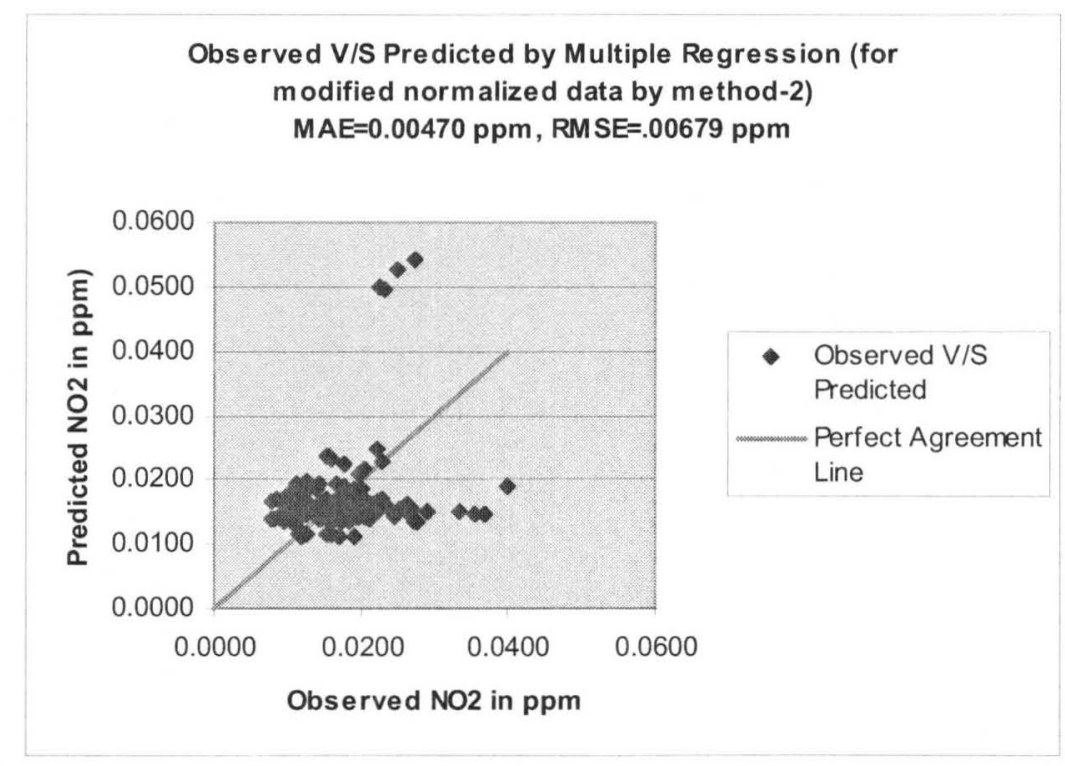

Figure 5.41

Observed and Predicted values by MR model for modified normalized data by method-2 


\subsubsection{Discussion}

The optimal ANN models (one for each set of data), defined here as those with the best or lowest MAE values, were used to predict the concentrations of $\mathrm{NO}_{2}$ in ambient air. The prediction or validation was performed by furnishing the ANN models with only the operationally available values of observation variables. With regard to the MR models too, the same set of input values were used so that a valid comparison between the two model types (ANN and MR) can be evaluated. The following section describes the notable observations derived from the $\mathrm{NO}_{2}$ modeling results.

- The values for MAE and RMSE are lower (better) for modified data as compared with use of the original data for both the model types-ANN and MR models - and it strengthens the concept that the removal of outliers improves model performance irrespective of modeling approach. Prediction with modified data was consistently better and more reliable.

- The graphical comparison of predicted and observed $\mathrm{NO}_{2}$ concentration closely follow one another in the case of both ANN and MR models, indicating the methods are well suited to the application and the approach can yield potentially useful results for research or planning applications. .

- Using quantitative measures of performance, the differences between predicted and observed $\mathrm{NO}_{2}$ values are lower in the case of ANN modeling, and this indicates an improved modeling approach relative to MR.

- The performance criteria values for both MAE and RMSE are small in the case of ANN models indicating both robustness and accuracy of ANN over MR models. 
- Scatter plots support the same fact - the ANN's supremacy over MR. Considering the data spread around the 1:1 line or "Perfect Agreement Line", it is less in the case of ANN, supporting the conclusion of superior model performance. This is also indicated by the lower values of RMSE in the case of ANN model relative to the MR model.

- The distribution in number of data points about the "Perfect Agreement Line" is equally distributed (above and below) in all the scatter plots indicating unbiased ANN model performance.

- The distribution of data point predictions shown in both the scatter plots and point comparisons of predicted and observed values are identical for the MR models using either method of data normalization. This result shows the data normalization method does not affect MR model and this fact is also supported by the values of MAE and RMSE being equal in MR modeling for both the methods of data normalization.

- But abovementioned fact is not applicable to ANN modeling as the plots and curves are different and the values of MAE and RMSE as well. The method of data normalization therefore does affect the ANN modeling and is a very crucial element to be decided by trial and error.

\subsubsection{Effect of ITS on ambient air quality}

\subsubsection{Results}

In this section, the effect of ITS on ambient air quality has been highlighted. Using trained network to learn about the effect of ITS on air quality (concentration of $\mathrm{NO}_{2}$ ), 
involved running the network with all of the input neurons, except the one of ITS, held constant; interactions could be explored by setting ITS at various values--starting from $0 \%$ through $100 \%$ with an increment of $2 \%$. As it can be seen from Figure 5.21 , five curves have been plotted - four for the four sets of data with ANN and one for the MR model.

\section{\%ITS V/S NO2 Concentration in ambient air}

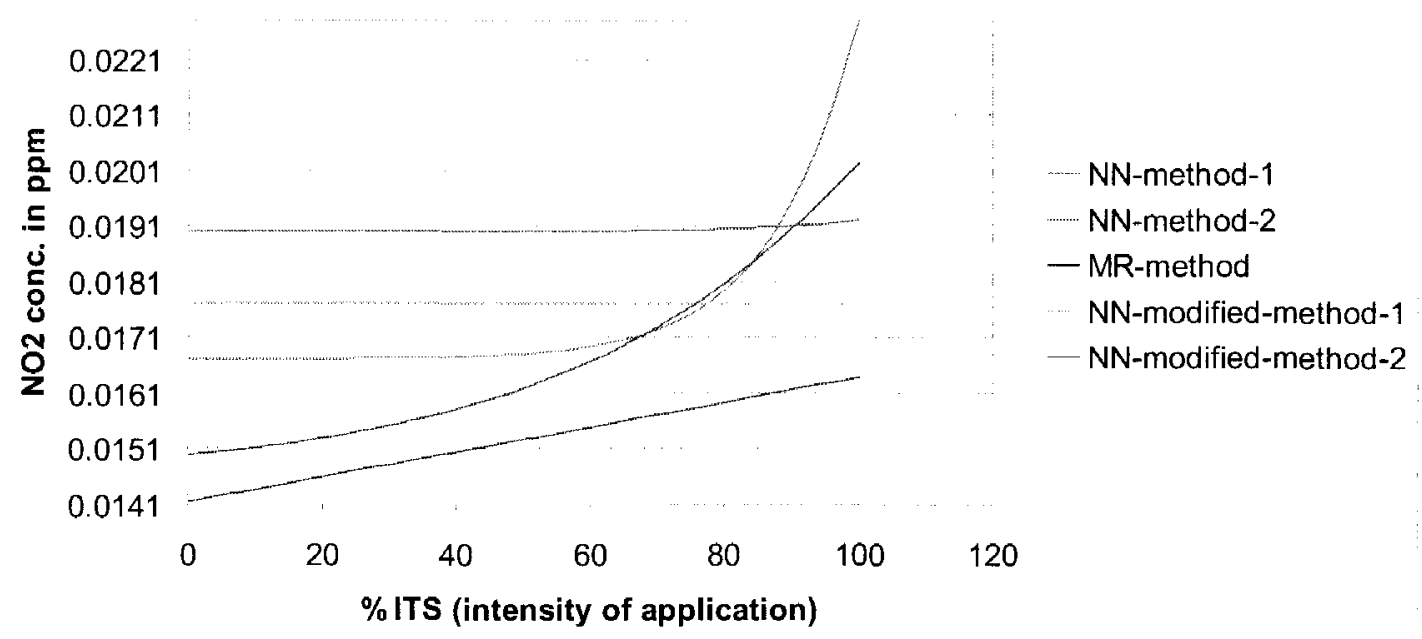

Figure 5.42

$\%$ ITS (intensity of application) versus $\mathrm{NO}_{2}$ concentration in ambient air

\subsubsection{Discussion}

Following section describes the important observations about the results of $\mathrm{NO}_{2}$ modeling with regard to ITS effects on air quality:

- Figure 5.42 depicts some exciting results regarding the variability of $\mathrm{NO}_{2}$ concentrations in ambient air with the intensity of application of ITS. Either the concentrations are increasing or remaining unaffected with ITS which indicates that the ITS is increasing or not affecting the concentration of 
$\mathrm{NO} 2$ in ambient air. It is a startling observation in the sense that it may create conformity problems for the entire transportation program - not just ITS projects.

- Induce demand increases pollution because more number of vehicles would be on the road due to smoothening of traffic by ITS.

- Since the average speed on roads is increased because of less

delay/congestion due to ITS, the rate of emission also increases that causes more pollution.

- In contrast, because of less congestion/delay, fucl saving takes place causing significant reduction in emissions of motor vehicles. This contributes in reduction of $\mathrm{NO}_{2}$ pollution in ambient air.

- Ovcrall ITS either does not help or worsens the $\mathrm{NO}_{2}$ pollution in ambient air as it evident from Figure 5.42. The reasons could be as follows: a) Induced demand due to the less congestion because of ITS (but it was not the case for $\mathrm{CO}$ as $\mathrm{CO}$ concentration reduced with ITS) b) NO2 emissions increase dramatically in large proportion as compared with $\mathrm{CO}$ as the speed of vehicles increase after a certain value and that might be the reason behind the difference between NO2 and CO implications. So the overall effect of induced demand plus increased emissions (due to average speed increase) minus less congestion/delay is the increase of ambient NO2 concentrations (though small, however an increase). It may lead to the serious implications with regard to the nonattainment areas. 


\subsection{Ozone $\left(\mathrm{O}_{3}\right)$}

Several configurations of ANN model structure (combinations of nodes and hidden layers) were implemented and applied using sets of $\mathrm{O}_{3}$ data--four sets altogether. These sets, like $\mathrm{CO} \& \mathrm{NO}_{2}$ data sets in previous sections, were as follows:

Set 1: Original data normalized by method-1 (n1),

Set 2: Modified data normalized by method-1 (n1),

Set 3: Original data normalized by method-2 (n2) and,

Set 4: Modified data normalized by method-2 (n2).

Here $\mathrm{n} 1$ and $\mathrm{n} 2$ indicate the two methods of data normalization as described in Chapter-IV.

\subsubsection{ANN Architecture v/s MAEs}

\subsubsection{Results}

In this section, the influence of $\mathrm{ANN}$ architecture on the MAE performance measures has been highlighted with regard to $\mathrm{O}_{3}$ modeling. Starting with very simple architecture of ANN and subsequently increasing the model structure complexity, MAE performance measures were determined corresponding to each model configuration or architecture. Also, a Multiple Regression study was performed on each of the four data sets and the corresponding MAE values were determined. Tables 5.9 through 5.12 summarize the model architecture configurations and corresponding values of MAE for each and Figure 5.43 through 5.46 depict the corresponding plots. Notations in this section are same as in the previous sections and the same is the description about the figures. Only difference is that this section deals with $\mathrm{O}_{3}$. 
Table 5.9 ANN model performance according to architecture and corresponding MAE measure (for normalized data by method-1)

Where:

\begin{tabular}{|c|c|}
\hline Configuration-n 1 & $\% \mathrm{E}(\mathrm{MAE})$ \\
\hline$(1-1-0)^{*}$ & 9.7 \\
\hline$(1-2-0)$ & 10.2 \\
\hline$(1-3-0)$ & 9.7 \\
\hline$(1-4-0)$ & 9.4 \\
\hline$(\mathbf{1 - 5}-0)$ & $\mathbf{8 . 7}$ \\
\hline$(1-6-0)$ & 9.0 \\
\hline$(2-1-1)$ & 9.0 \\
\hline$(2-2-2)$ & 9.9 \\
\hline$(2-4-4)$ & 9.2 \\
\hline$(2-1-2)$ & 9.7 \\
\hline$(2-3-2)$ & 9.9 \\
\hline Multiple Regression & 11.5 \\
\hline
\end{tabular}

Meanings of various terms and notations are the same as mentioned in the previous sections.

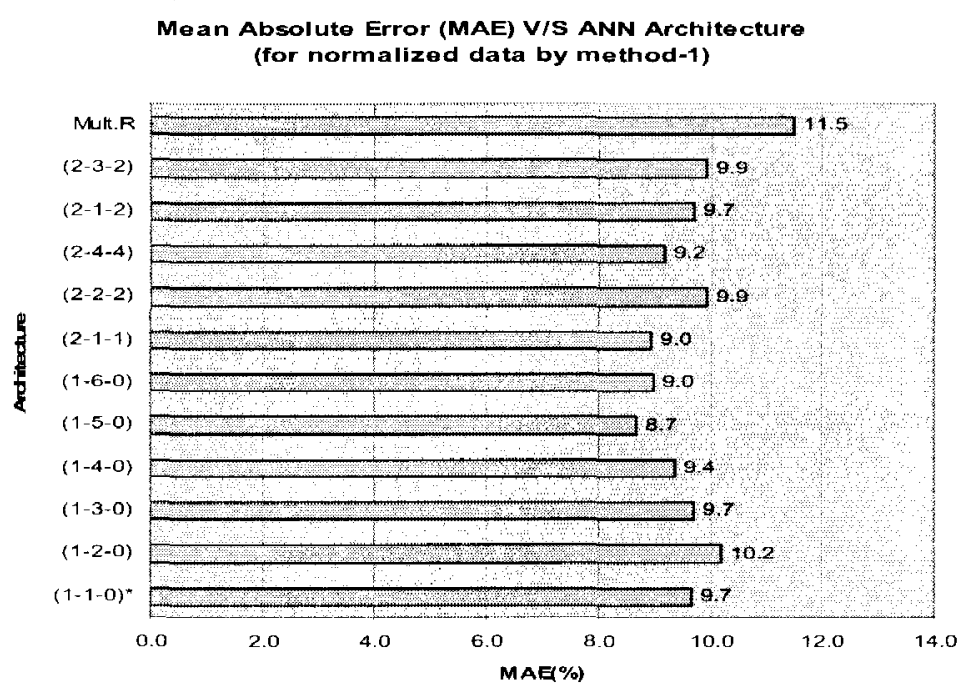

Figure 5.43

ANN performance summary according to architecture and corresponding MAE (for normalized data by method-1) 
Table 5.10 ANN model performance according to architecture and corresponding MAE measure (for modified normalized data by method-1)

\begin{tabular}{|c|c|}
\hline Configuration-n l-m & $\% \mathrm{E}(\mathrm{MAE})$ \\
\hline$(1-1-0)$ & 6.8 \\
\hline$(1-2-0)$ & 7.9 \\
\hline$(1-3-0)$ & 5.7 \\
\hline$(1-4-0)$ & $\mathbf{5 . 6}$ \\
\hline$(1-5-0)$ & 13.3 \\
\hline$(1-6-0)$ & 7.0 \\
\hline$(2-1-1)$ & 6.1 \\
\hline$(2-2-2)$ & 6.0 \\
\hline$(2-3-3)$ & 7.5 \\
\hline$(2-4-4)$ & 7.3 \\
\hline$(2-1-2)$ & 6.4 \\
\hline Multiple Regression & 9.4 \\
\hline
\end{tabular}

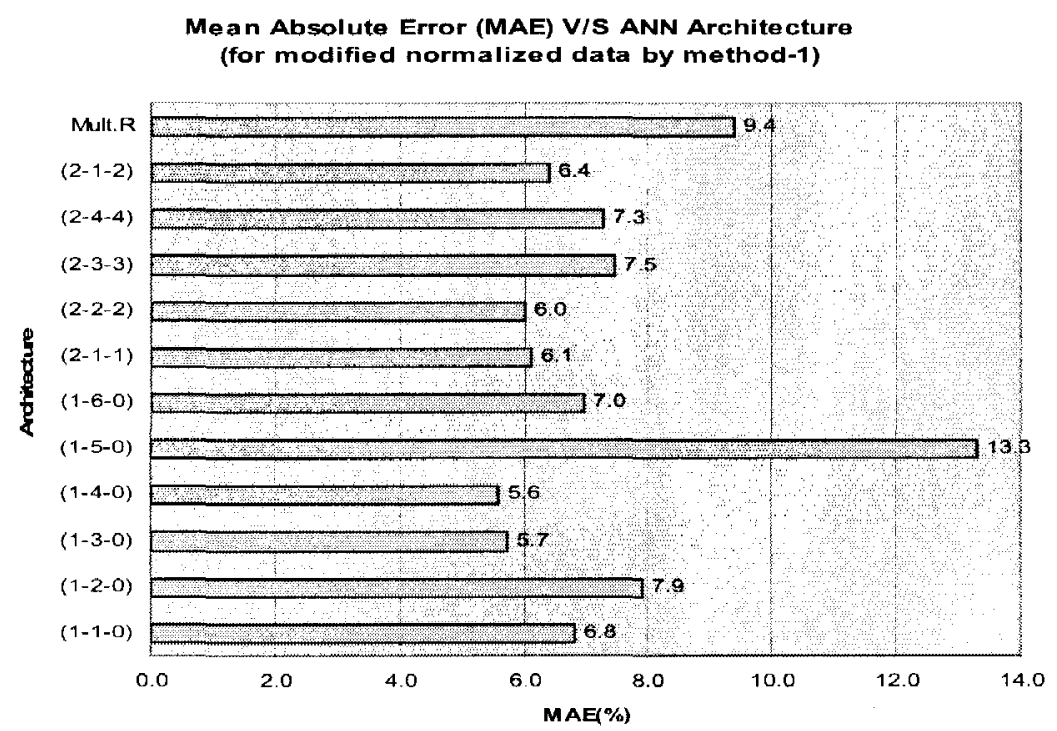

Figure 5.44

ANN performance summary according to architecture and corresponding

MAE (for modified normalized data by method-1) 
Table 5.11 ANN model performance according to architecture and corresponding MAE measure (for normalized data by method-2)

\begin{tabular}{|c|c|}
\hline Configuration-n2 & $\begin{array}{c}\% \mathrm{E} \\
\text { (MAE) }\end{array}$ \\
\hline $\mathbf{( 1 - 1 - 0 )}$ & $\mathbf{9 . 1}$ \\
\hline$(\mathbf{1 - 2 - 0 )}$ & $\mathbf{9 . 1}$ \\
\hline$(1-3-0)$ & 11.0 \\
\hline$(1-4-0)$ & 10.9 \\
\hline$(2-1-1)$ & 9.5 \\
\hline$(2-2-2)$ & 9.9 \\
\hline$(2-3-3)$ & 9.8 \\
\hline$(2-2-1)$ & 9.3 \\
\hline$(2-3-2)$ & 9.6 \\
\hline $\begin{array}{c}\text { Multiple } \\
\text { Regression }\end{array}$ & 11.5 \\
\hline
\end{tabular}

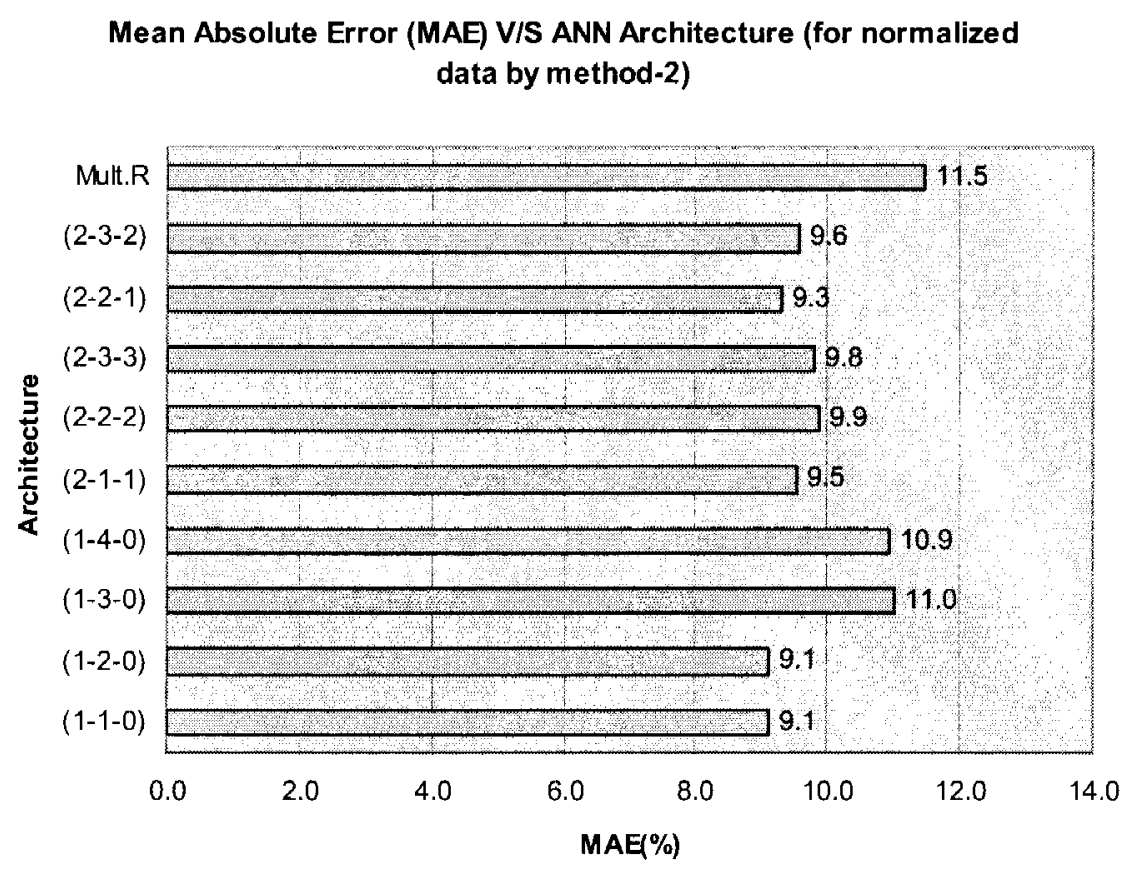

Figure 5.45

ANN performance summary according to architecture and corresponding

MAE (for normalized data by method-2) 
Table 5.12 ANN model performance according to architecture and corresponding MAE measure (for modified normalized data by method-2)

\begin{tabular}{|c|c|}
\hline Configuration-n2-m & $\% \mathrm{E}$ \\
\hline$(1-1-0)$ & 6.8 \\
\hline$(1-2-0)$ & 5.9 \\
\hline$(\mathbf{1 - 3 - 0 )}$ & $\mathbf{5 . 6}$ \\
\hline$(1-4-0)$ & 6.4 \\
\hline$(2-1-2)$ & 11.5 \\
\hline$(2-1-1)$ & 6.3 \\
\hline$(2-3-3)$ & 7.0 \\
\hline$(2-2-1)$ & 6.7 \\
\hline Multiple Regression & 9.4 \\
\hline
\end{tabular}

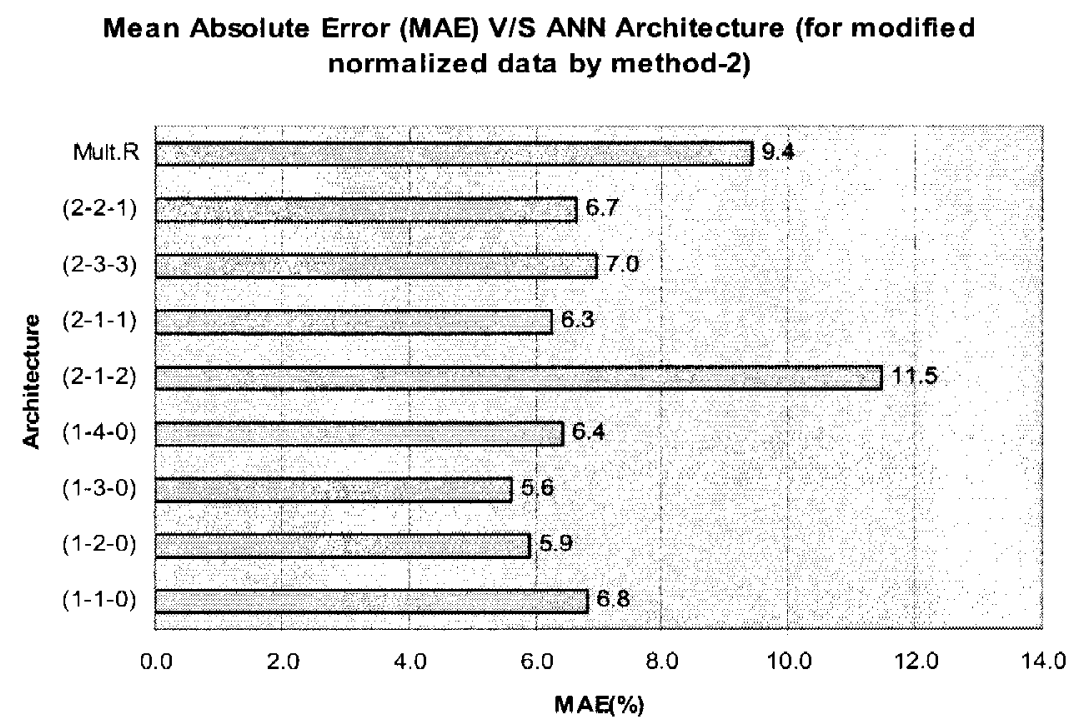

Figure 5.46

ANN performance summary according to architecture and corresponding

MAE (for modified normalized data by method-2) 


\subsubsection{Discussion}

Like $\mathrm{CO}$ and $\mathrm{NO}_{2}$ modeling mentioned in previous sections, several ANN model configurations or architectures were applied with $\mathrm{O}_{3}$ data in order to arrive at the optimal form to minimize MAE. MAE is a standard performance measure to check the capability of a prediction model.

Entries in bold in the tables indicate the configuration of ANN with the lowest MAE in prediction. Following section summarizes the significant observations about the results of $\mathrm{O}_{3}$ modeling with regard to variations in ANN Architecture.

- The values of MAE for Set 1 data varied from $8.7 \%$ corresponding to ANN model configuration (1-5-0) to $10.2 \%$ corresponding to ANN model configuration (1-2-0) and $11.5 \%$ for Multiple Regression modeling.

- The MAE values for Set 2 data varied from $5.6 \%$ corresponding to ANN model configuration (1-4-0) to $13.3 \%$ corresponding to ANN model configuration (1-5-0) and $9.4 \%$ for Multiple Regression modeling.

- The MAE values for Set 3 data varied from $9.1 \%$ corresponding to ANN model configuration (1-1-0) to $11.0 \%$ corresponding to ANN model configuration (1-3-0) by and $11.5 \%$ for Multiple Regression modeling.

- The MAE values for Set 4 data varied from $5.6 \%$ corresponding to ANN model configuration (1-3-0) to $11.5 \%$ corresponding to ANN model configuration (2-1-2) by and $9.4 \%$ for Multiple Regression modeling. 
- The values of MAE with Multiple Regression are identical for Set 1 \& Set 3 $(11.5 \%)$ and also for Set 2 and Set $4(9.4 \%)$ indicating that the method of normalization does not affect the results of multiple regression modeling.

- From Table 5.9 through 5.12, it is evident that MAE values are larger when the ANN model is either under-trained or over-trained; however there is no clear-cut rule by which one can define the optimal ANN structure. Although the tendency is for more complex the relationships between dependent and independent variables require more complex ANN structures, the final details of model structure must still be determined using an iterative, trial and error style procedure. Through the training or model calibration process one must monitor the ANN evolution and avoid both under-trained and over-trained regions of the solution space.

- The MAE values in prediction application for $\mathrm{O}_{3}$ vary from $5.6 \%$ to $9.1 \%$ for these sets and those are quite justifiable. Here one point is noteworthy and that is about the MAE values in prediction of $\mathrm{O} 3$ concentrations are far below than that of $\mathrm{CO}$ and $\mathrm{NO}_{2}$ indicating the close association of $\mathrm{O} 3$ (ground level) with the transportation parameters or variables as compared with $\mathrm{CO}$ and $\mathrm{NO}_{2}$. This result is contrary to what is generally believed (that the contribution of transportation sector to $\mathrm{CO}$ in ambient air is maximum percentagewise as compared with other priority pollutant and this factor should be investigated in detail as there is a confusion about the level of NOx may increase, decrease or have no effect on area's Ozone level since it apparently depends on the proportion of NOx and VOC). 
- The Lowest MAE for Set 1 data was $8.7 \%$ and $5.6 \%$ for Set 2 (modified data by removing outliers) indicating that the by removing "outliers" the prediction error can be reduced significantly. The same result was found for the Set 3 and 4 data as well and also for the multiple-regression modeling. Removal of outliers therefore always improves the accuracy of any prediction model.

- The lowest value of MAE for data Set 1 (normalized by method-1, n1) was $8.7 \%$ and was $9.1 \%$ for Set 3 (normalized by method-2, n2). Similarly, the lowest MAE for data Set-2 (normalized by method-1, n1) was $5.6 \%$ and was the same (5.6\%) for Set 4 (normalized by method-2, n2). It indicates that the method of normalization significantly influence the results of ANN modeling and in this particular study, method number one (n1) proved to be providing more accurate prediction as compared with the method number two (n2).

\subsubsection{Comparison of Observed and Predicted values}

\subsubsection{Results}

Observed and predicted results from both the models-ANN model and Multiple Regression (MR) model-were compared using values for both the MAE and RMSE (for the $\mathrm{O}_{3}$ parameter). The model configuration or architecture providing the least MAE result was selected as the "target" or optimal model and served to provide the predicted values for model validation evaluation. By using 10 -fold technique, the predicted values were found for both the models, the optimal ANN and multiple regression. To provide a 
means for visualization of the results, a series of curves were plotted comparing observed and predicted values of $\mathrm{O}_{3}$ concentration. Additionally, a series of scatter plots were developed to illustrate the statistical comparison relative to the perfect agreement line and provide an indication of model bias if any. Figures 5.47 through 5.54 depict the plots of observed and predicted for original data and Figures 5.55 through 5.62 show the plots between observed and predicted for modified data. 


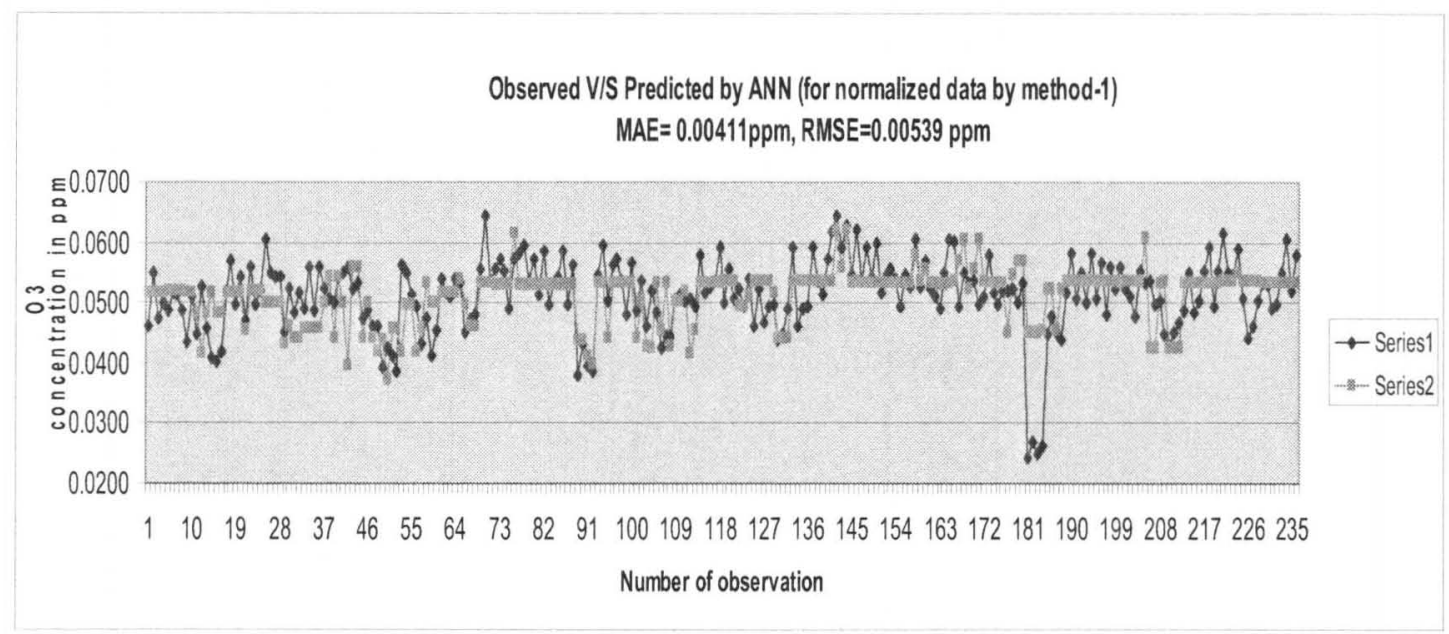

Figure 5.47

Observed versus Predicted values by ANN model for normalized data by method-1

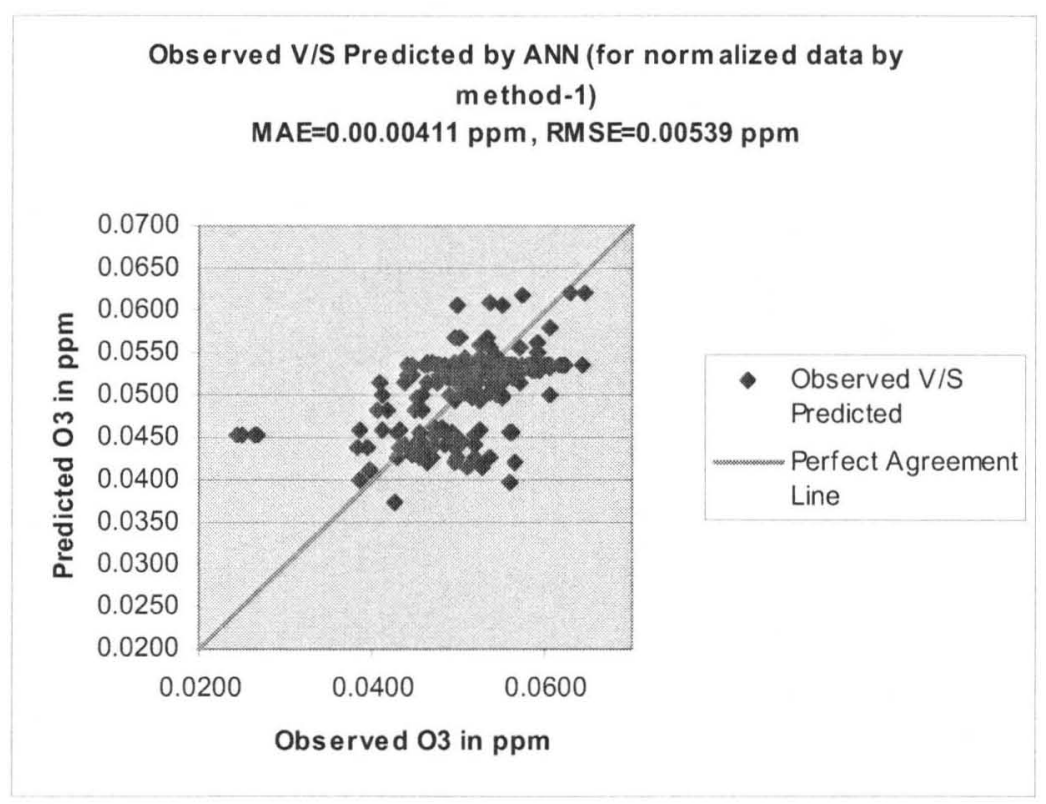

Figure 5.48

Observed and Predicted values by ANN model for normalized data by method-1 


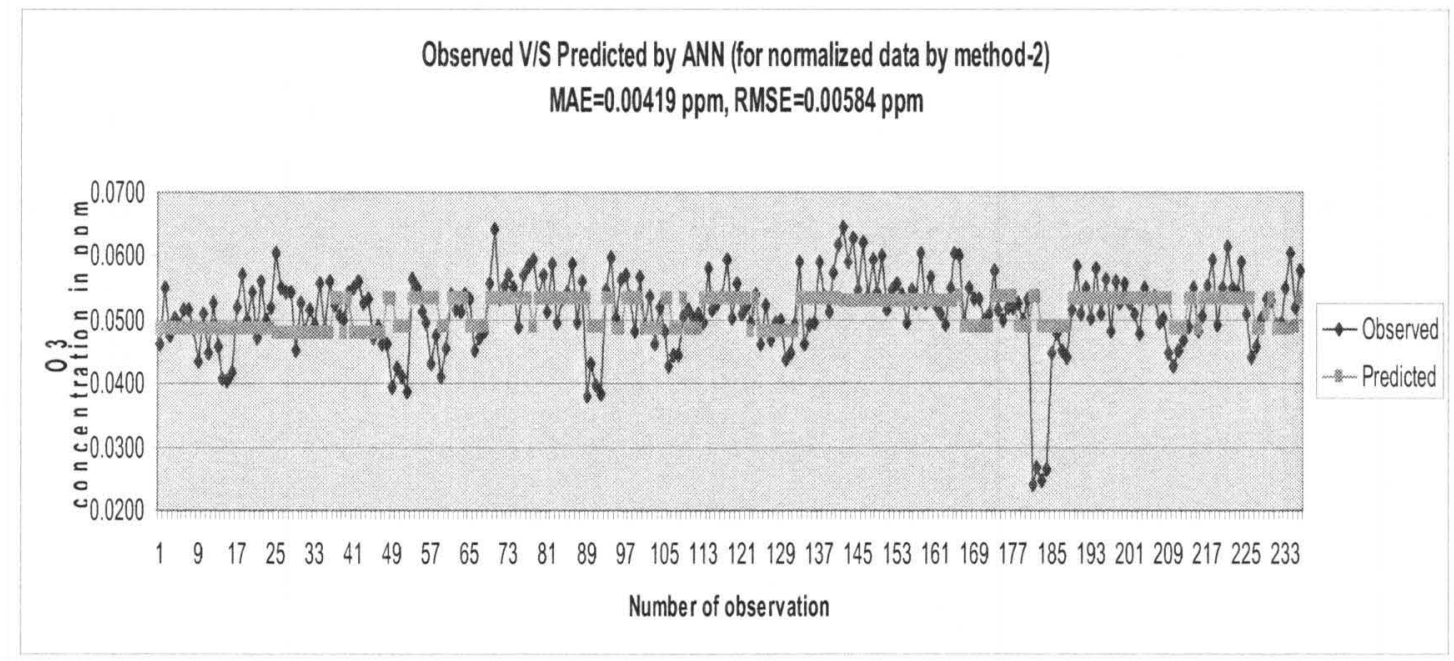

Figure 5.49

Observed versus Predicted values by ANN model for normalized data by method-2

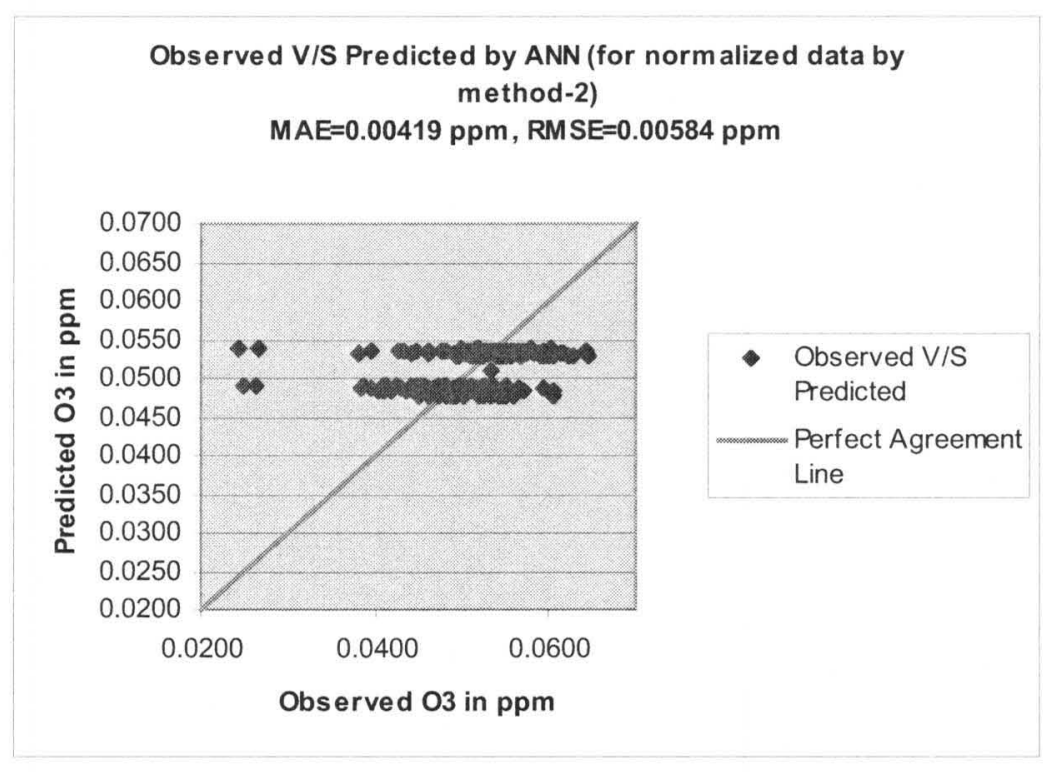

Figure 5.50

Observed and Predicted values by ANN model for normalized data by

method-2 


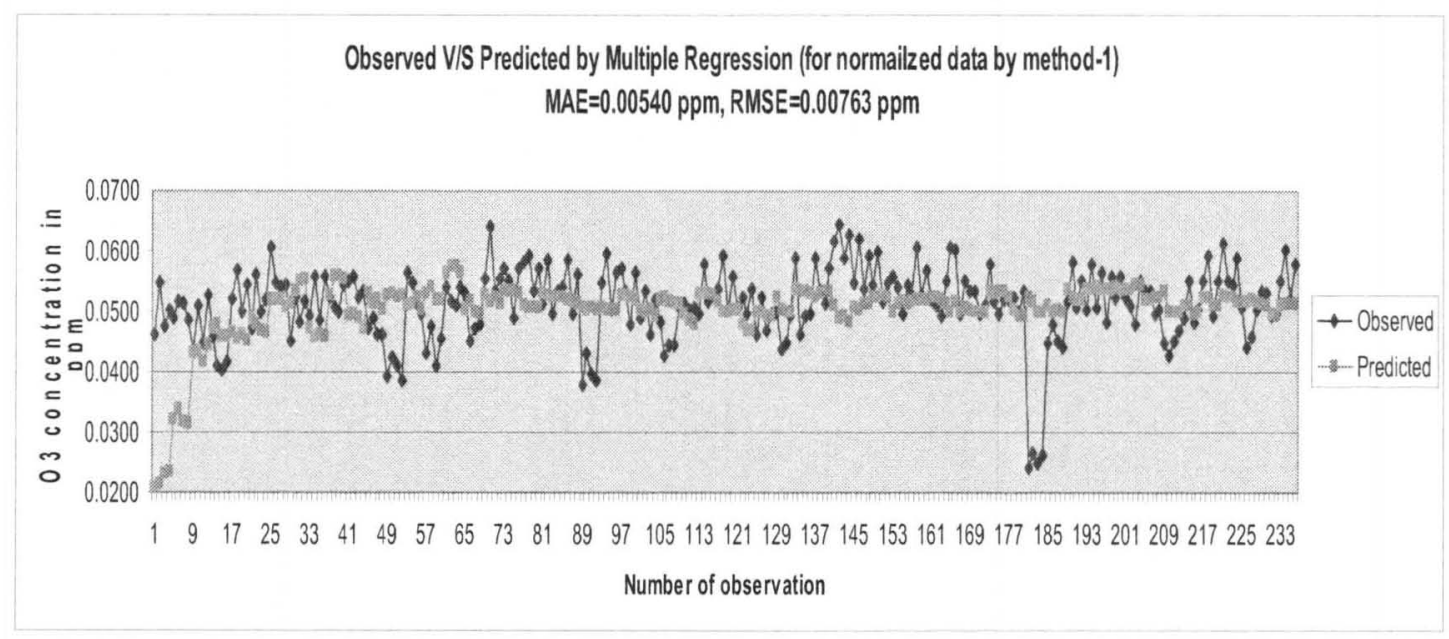

Figure 5.51

Observed versus Predicted values by MR model for normalized data by method-1

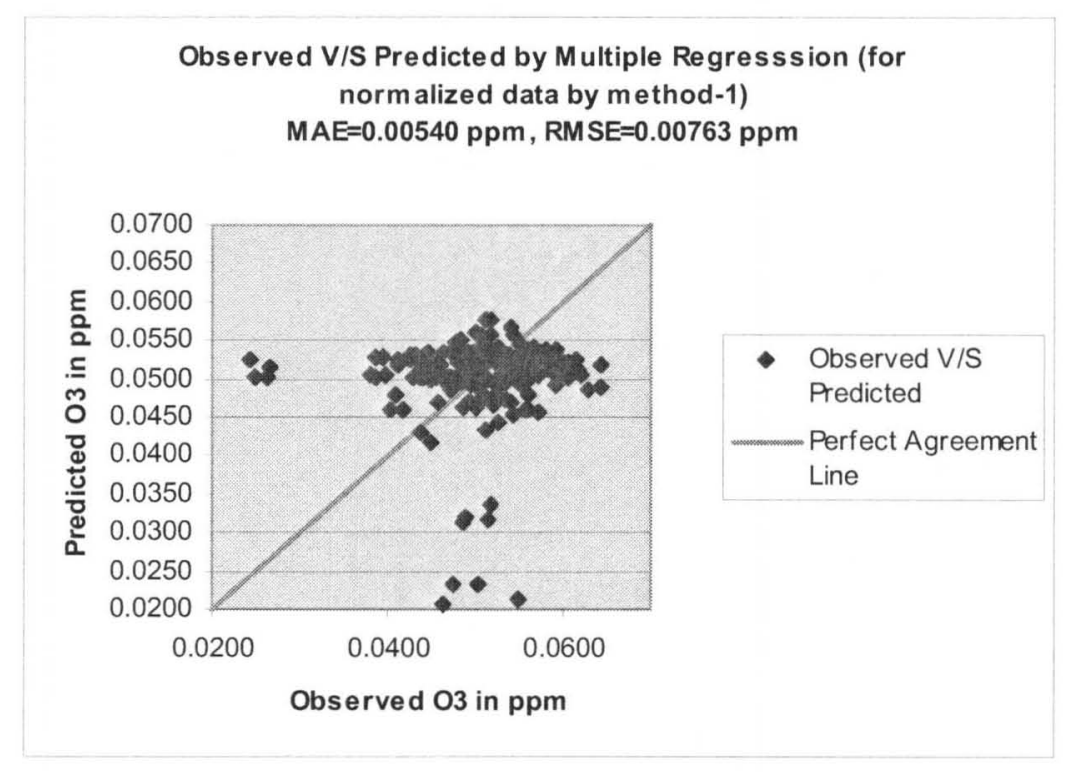

Figure 5.52

Observed and Predicted values by MR model for normalized data by

method-1 


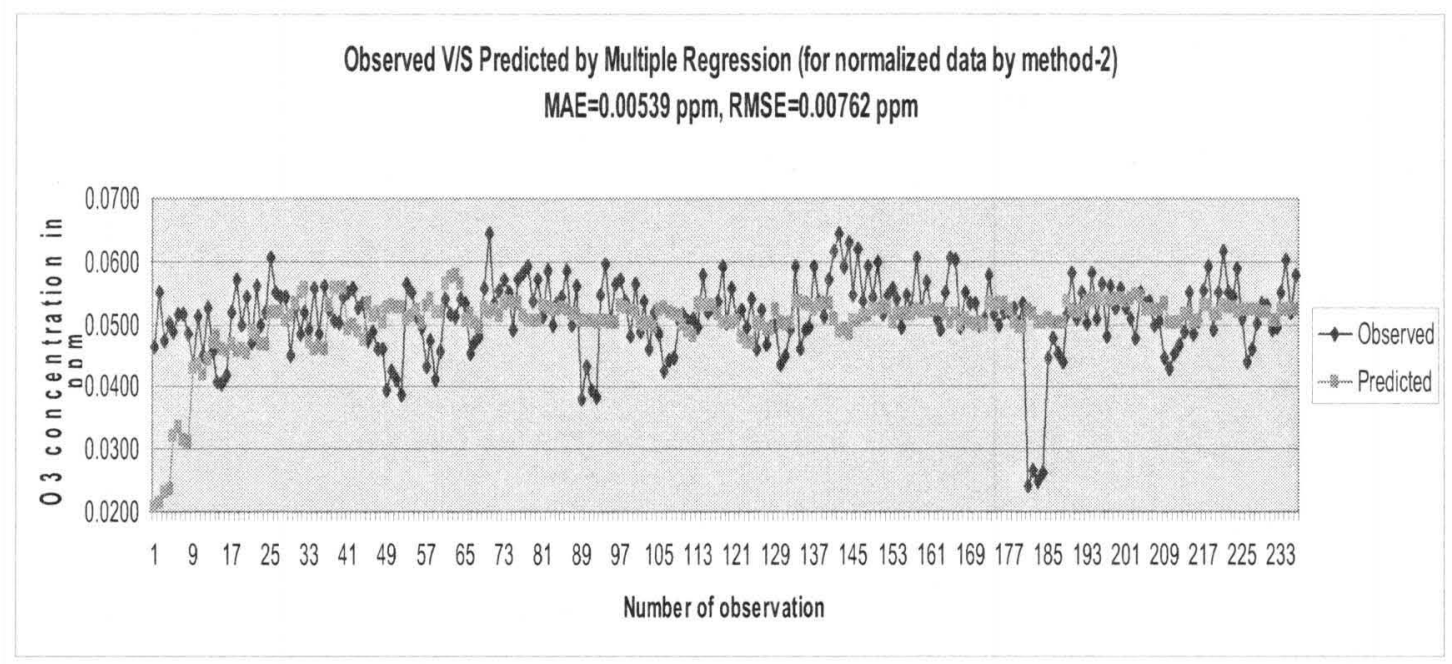

Figure 5.53

Observed versus Predicted values by MR model for normalized data by method-2

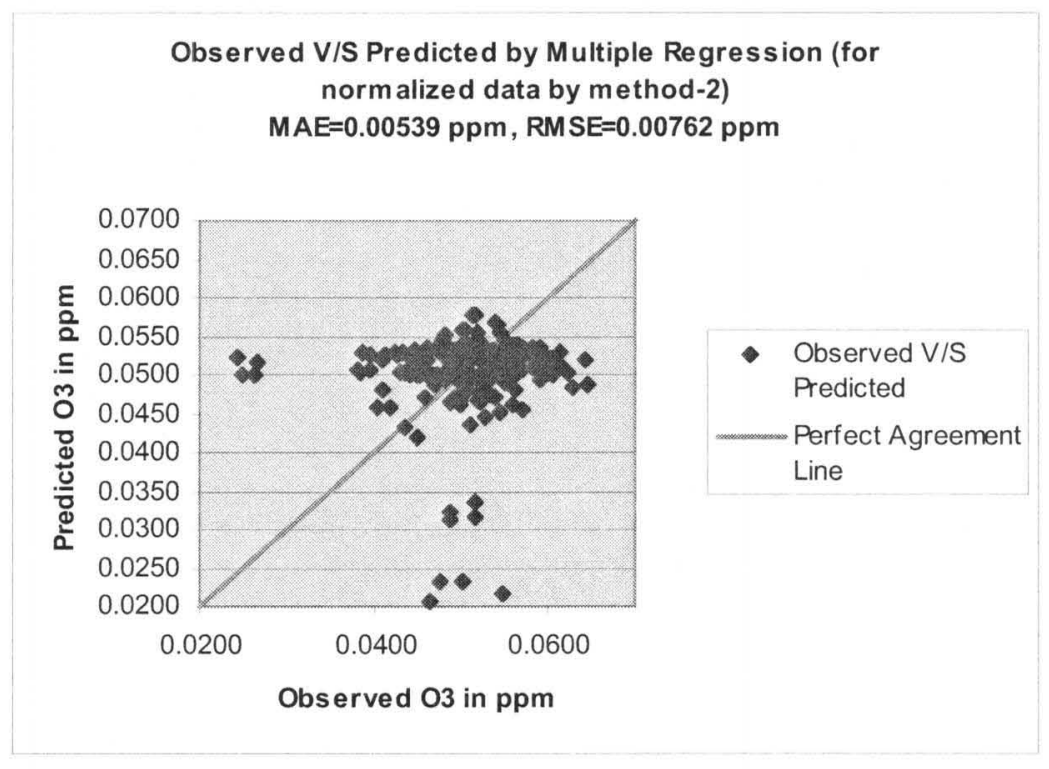

Figure 5.54

Observed and Predicted values by MR model for normalized data by method-2 


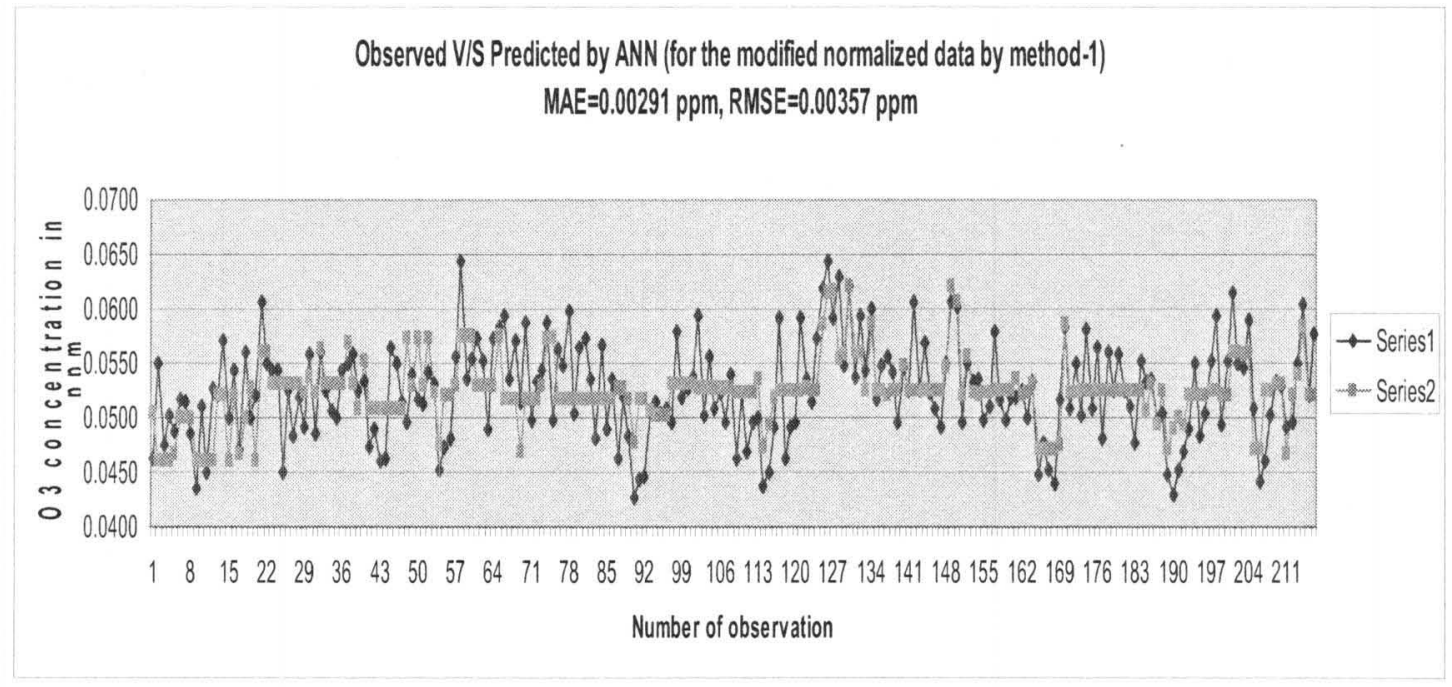

Figure 5.55

Observed versus Predicted values by ANN model for modified normalized

data by method-1

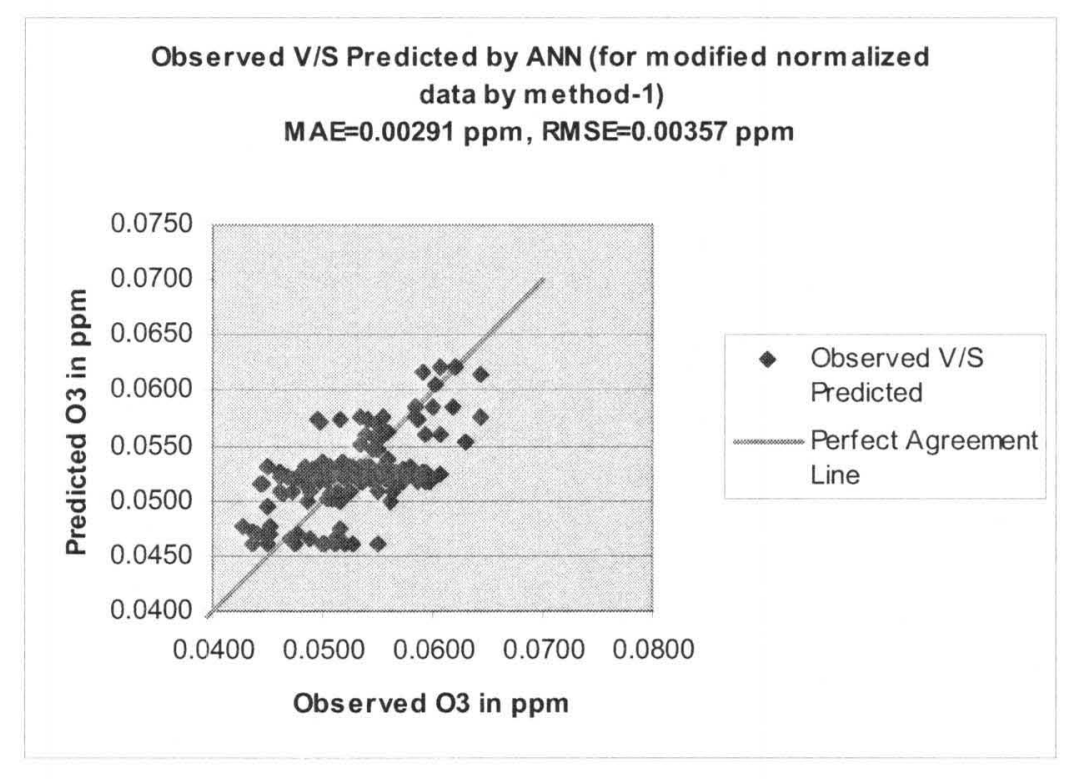

Figure 5.56

Observed and Predicted values by ANN model for modified normalized data by method-1 
Observed VIS Predicted by ANN (for modified normalized data by method-2)

MAE $=0.00293 \mathrm{ppm}$, RMSE $=0.00365 \mathrm{ppm}$

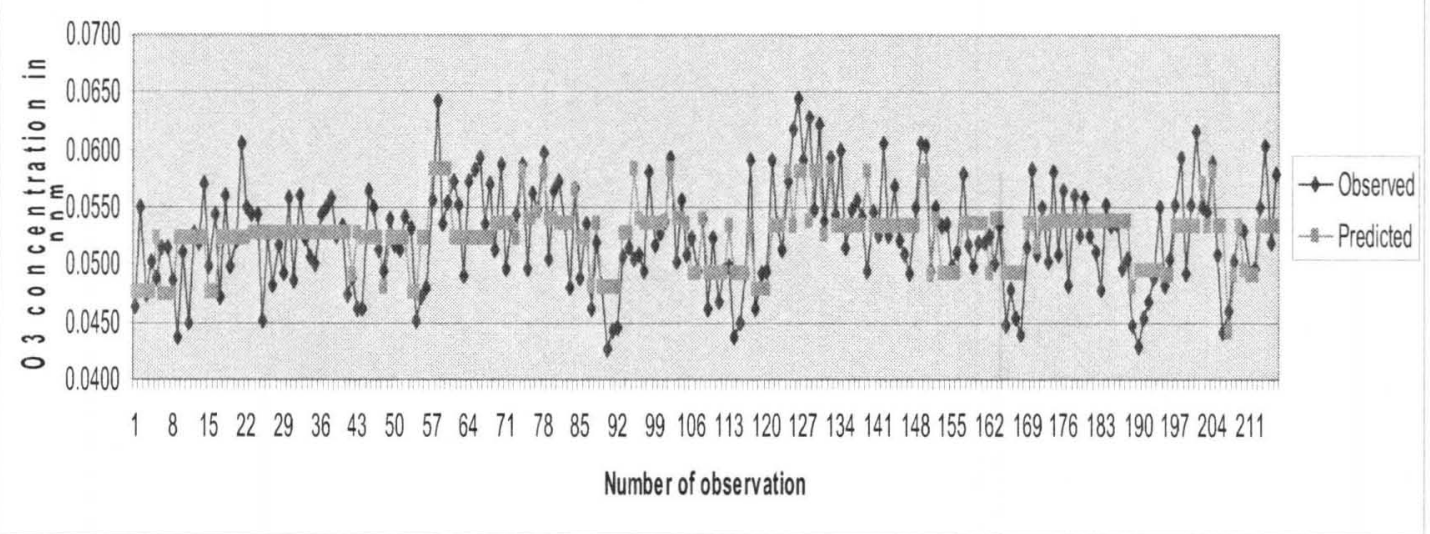

Figure 5.57

Observed versus Predicted values by ANN model for modified normalized data by method-2

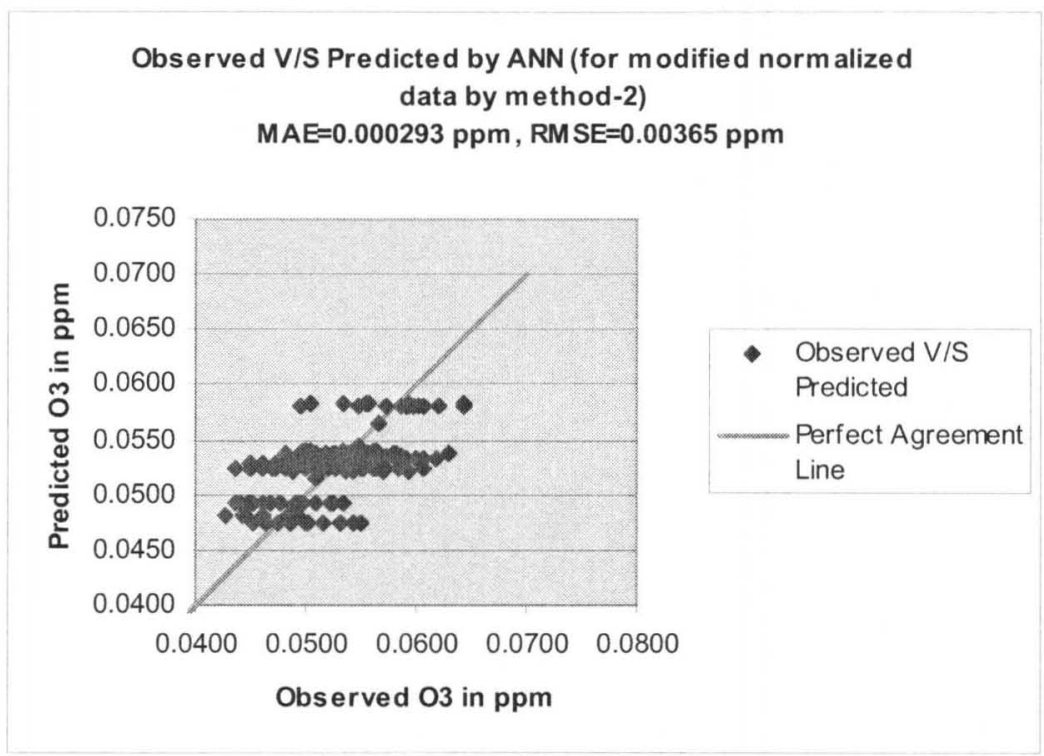

Figure 5.58

Observed and Predicted values by ANN model for modified normalized data by method-2 


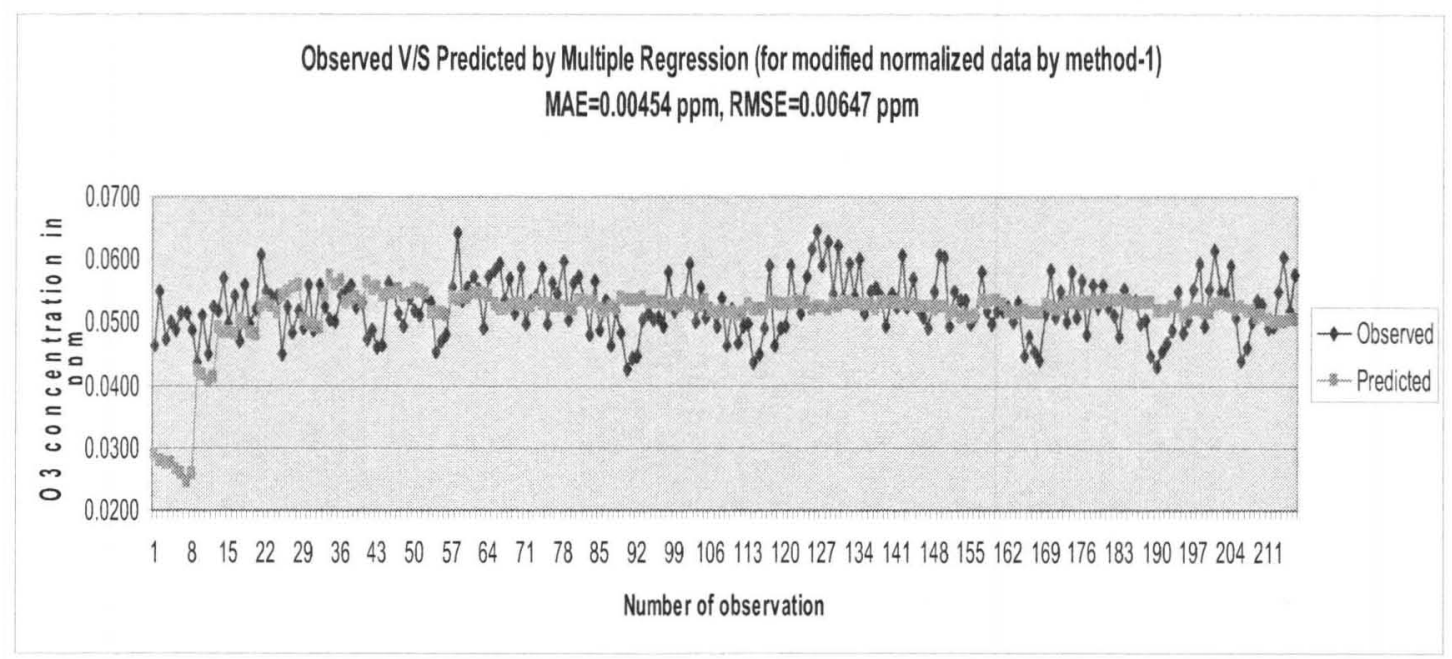

Figure 5.59

Observed versus Predicted values by MR model for modified normalized data by method-1

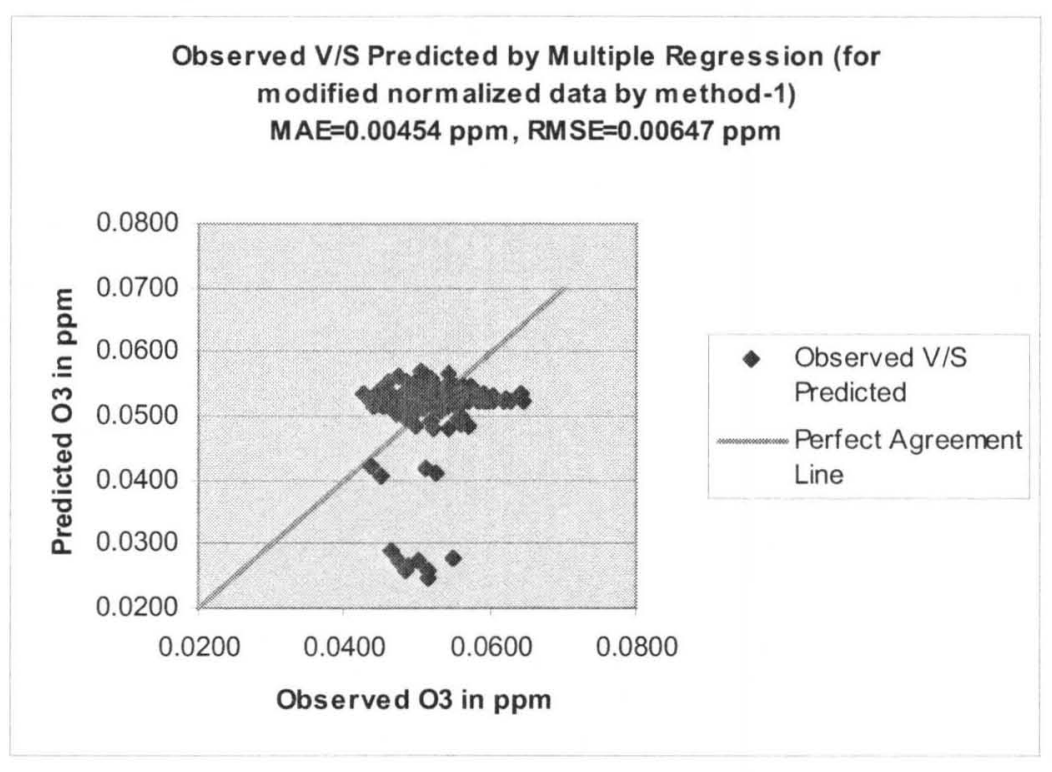

Figure 5.60

Observed and Predicted values by MR model for modified normalized data by method-1 


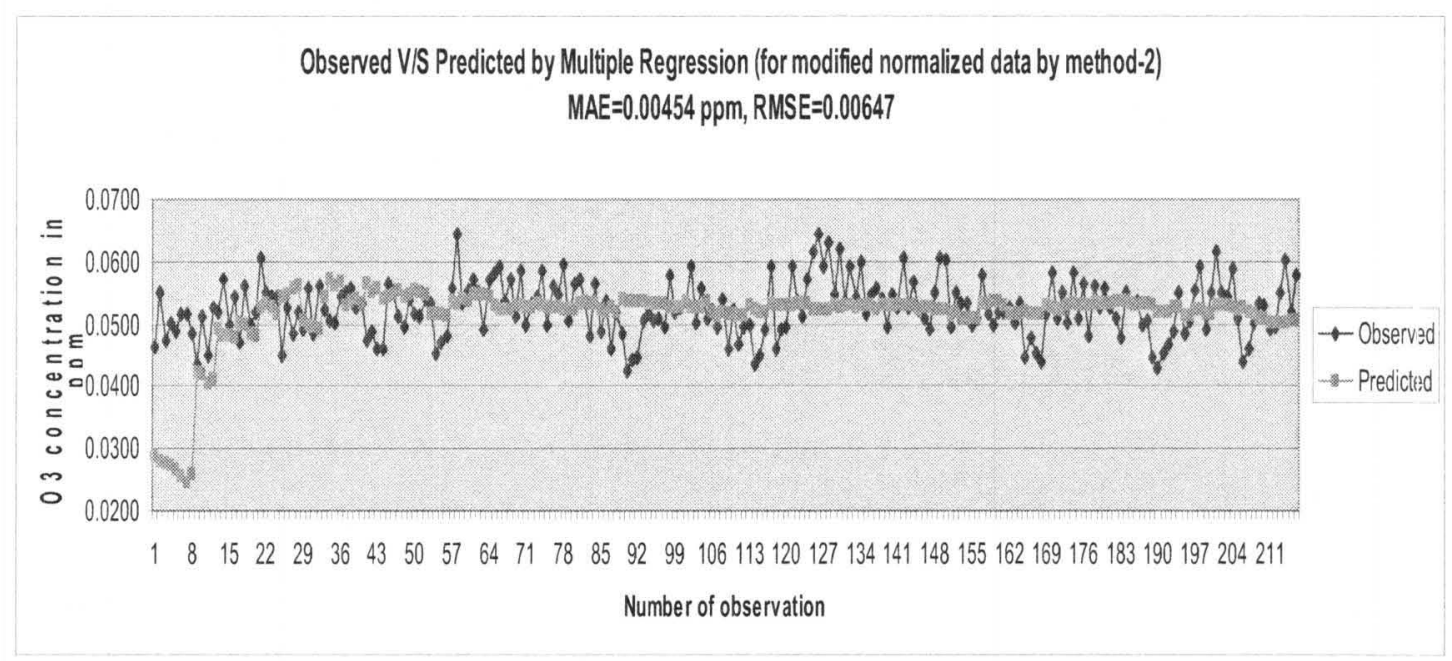

Figure 5.61

Observed versus Predicted values by MR model for modified normalized data by method-2

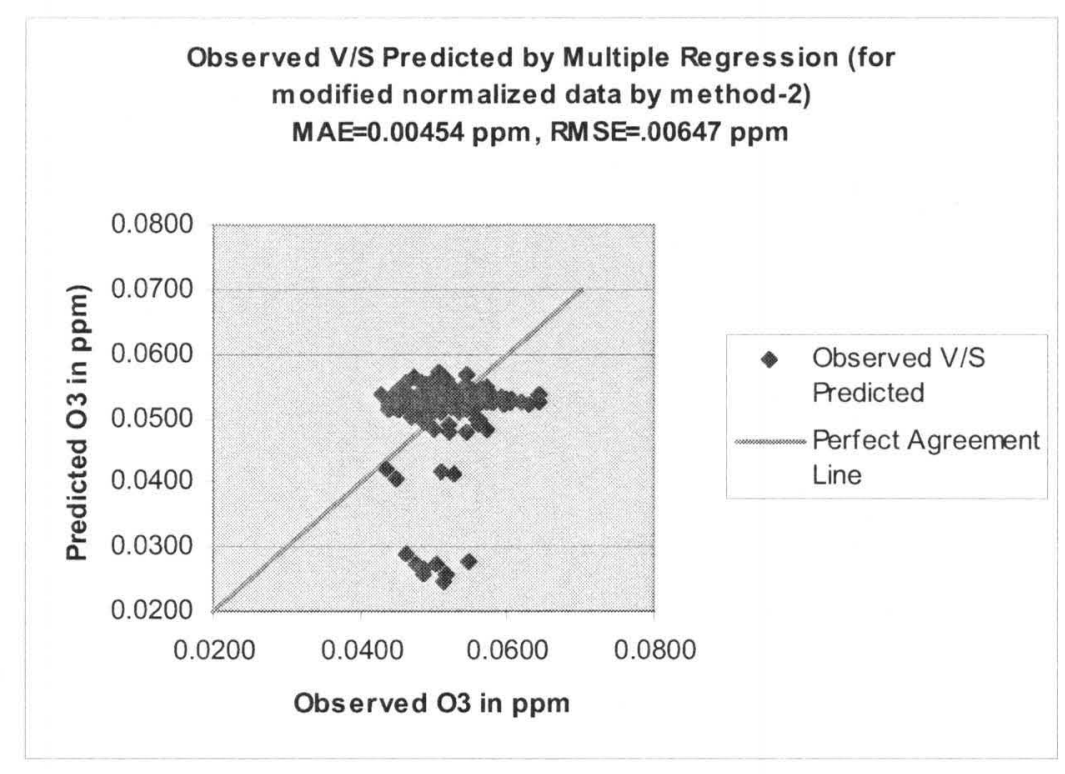

Figure 5.62

Observed and Predicted values by MR model for modified normalized data by method-2 


\subsubsection{Discussion}

With regard to $\mathrm{O}_{3}$ modeling also, the discussion is on the similar lines as it was with respect to $\mathrm{CO}$ and $\mathrm{NO}_{2}$. The optimal $\mathrm{ANN}$ models (one for each set of data), defined here as those with the best or lowest MAE values, were used to predict the concentrations of $\mathrm{NO}_{2}$ in ambient air. The prediction or validation was performed by furnishing the ANN models with only the operationally available values of observation variables. With regard to the MR models too, the same set of input values were used so that a valid comparison between the two model types (ANN and MR) can be evaluated. The following section describes the notable observations derived from the $\mathrm{O}_{3}$ modeling results.

- The values for MAEs and RMSEs are lower (better) for modified data as compared with use of the original data for both the model types-ANN and MR models - and it strengthens the concept that the removal of outliers improves model performance irrespective of modeling approach. Prediction with modified data was consistently better and more reliable.

- The graphical comparison of predicted and observed $\mathrm{O}_{3}$ concentration closely follow one another in the case of both ANN and MR models, indicating the methods are well suited to the application and the approach can yield potentially useful results for research or planning applications. .

- Using quantitative measures of performance, the differences between predicted and observed $\mathrm{O}_{3}$ values are lower in the case of ANN modeling, and this indicates an improved modeling approach relative to MR. 
- The performance criteria values for both MAE and RMSE are less in the case of ANN models indicating both robustness and accuracy of ANN over MR models.

- Scatter plots support the same fact -- the ANN's supremacy over MR. Considering the data spread around the 1:1 line or "Perfect Agreement Line", it is less in the case of ANN, supporting the conclusion of superior model performance. This is also indicated by the lower values of RMSE in the case of ANN model relative to the MR model.

- The distribution in number of data points about the "Perfect Agreement Line" is equally distributed (above and below) in all the scatter plots indicating unbiased ANN model performance.

- The distribution of data point predictions shown in both the scatter plots and point comparisons of predicted and observed values are identical for the MR models using either method of data normalization. This result shows the data normalization method does not affect MR model and this fact is also supported by the values of MAEs and RMSEs being equal in MR modeling for both the methods of data normalization.

- But abovementioned fact is not applicable to ANN modeling as the plots and curves are different and the values of MAEs and RMSEs as well. The method of data normalization therefore does affect the ANN modeling and is a very crucial element to be decided by trial and error. 
- RMSEs are more sensitive as compared with MAE to the large differences and that is the reason that rate of increase in RMSEs is more than the rate of MAEs.

\subsubsection{Effect of ITS on ambient air quality}

\subsubsection{Results}

In this section, the effect of ITS on ambient air quality has been highlighted. Using trained network to learn about the effect of ITS on air quality (concentration of $\mathrm{O}_{3}$ ), involved running the network with all of the input neurons, except the one of ITS, held constant; interactions could be explored by setting ITS at various values - starting from $0 \%$ through $100 \%$ with an increment of $2 \%$. As it can be seen from Figure 5.21 , five curves have been plotted-four for the four sets of data with ANN and one for the MR model.

\section{\%TS V/S O3 Concentration in ambient air}

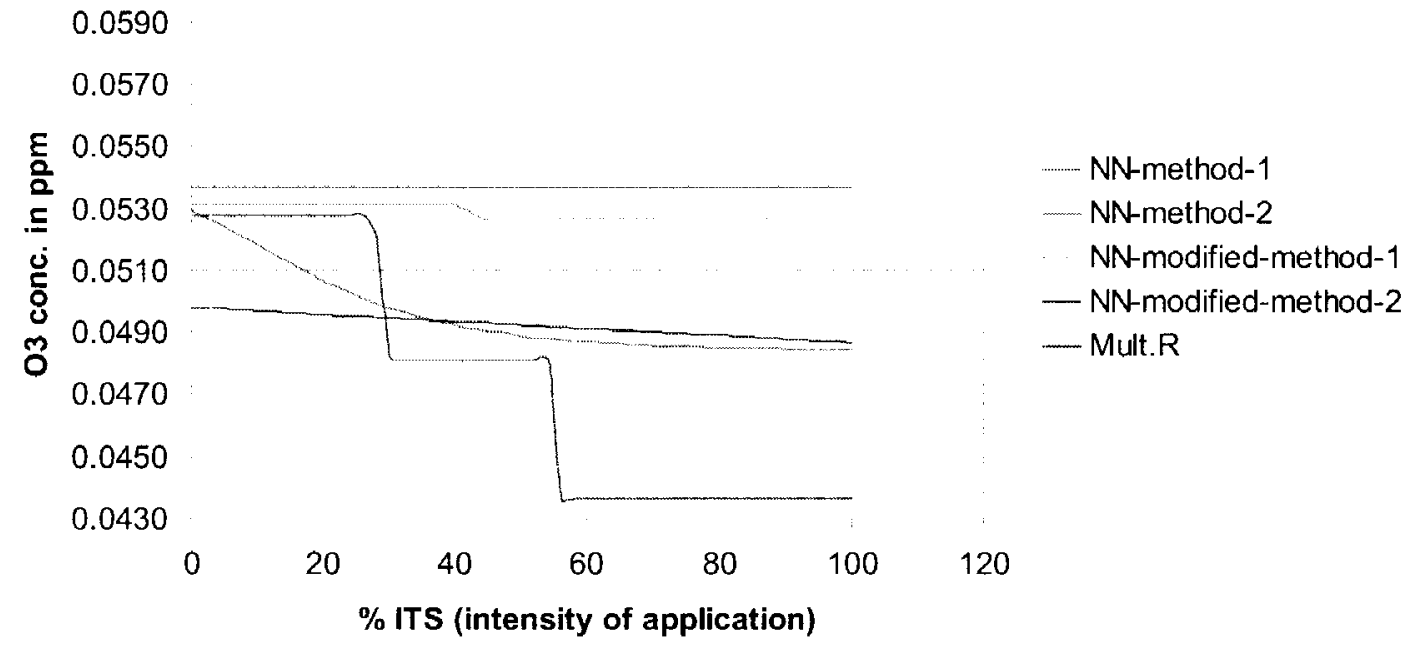

Figure 5.63

$\%$ ITS (intensity of application) versus $\mathrm{O}_{3}$ concentration in ambient air 


\subsubsection{Discussion}

Following section describes the important observations about the results of $\mathrm{O} 3$ modeling with regard to ITS effects on air quality:

- As it can be seen from Figure 5.63, Concentration of ground level O3 decreases with increase in the intensity of ITS application (on expected lines).

- Induce demand increases pollution because more number of vehicles would be on the road due to smoothening of traffic by ITS.

- Since the average speed on roads is increased because of less delay/congestion due to ITS, the rates of emission of $\mathrm{NO}_{\mathrm{x}}$ and VOC also increase that causes more Ozone pollution since Ozone is the result of a complex interaction between $\mathrm{NO}_{\mathrm{x}}$ and VOC.

- In contrast, because of less congestion/delay, fuel saving takes place causing significant reduction in emissions of motor vehicles. This may contribute in reduction of $\mathrm{O}_{3}$ pollution in ambient air.

- Overall ITS does help in curbing the $\mathrm{O}_{3}$ pollution in ambient air as it evident from Figure 5.63. Reason might be, "the cumulative effect of induced demand, effect of increase of average speed on emission rates of NOx and VOCs, and reduction of congestion/delay results in the reduction of $\mathrm{O} 3$ concentration which is a good thing".

The goal of this research was to devise a mechanism to quantify the air quality benefits of ITS and subsequently to identify the relationship between ITS and ambient air quality by using ANN modeling. This research has been successful in doing so and the 
results were well on the expected lines except a few which may not go very well with the prevailed notions. Also the results provided and strengthened some of the concepts about ANN and offered an insight and a starting point in the direction of quantification of ITS effects on air quality.

Next chapter consists of conclusions of this research. Also it consists of a few recommendations for further research on this topic. 


\section{CHAPTER VI}

\section{CONCLUSIONS AND RECOMMENDATIONS}

A summary of the findings and conclusions of this research is provided in this chapter. The main goal of the research was to devise a mechanism to quantify the air

quality benefits of Intelligent Transportation Systems (ITS) and then to identify the relationship between ITS and ambient air quality. To accomplish this goal, a plan was developed which included data mining from available operation resources and implementation of relational modeling methods, including both regression and artificial neural network (ANN) modeling.

\subsection{Conclusions}

Existing research studies were aimed primarily to quantify the effects of individual ITS applications on emissions, but to date, no research has been oriented towards determining the effect of ITS as a whole on the ambient air quality in urban regions. There have been no efforts to address the issues of induced demand, mobility conditions, and average speed increase with regard to ITS implementation and further, the quantification of these issues through modeling. This research provided a new approach incorporating and illustrating innovative methods to quantify and model the impact of these ITS issues, and to assess the effect of ITS on air quality. 
Based on the research completed in this dissertation, the following conclusions can be made:

- The method of data normalization does not affect the results of multiple regression modeling.

- Method of data normalization was found to have significant affect on the performance of the ANN model. In this particular study, method number one $(\mathrm{n} 1)$ proved to be giving more accurate prediction as compared with the method number two (n2).

- Removing "outliers" can reduce the prediction crror significantly.

Prediction with modified data results in better accuracy and improved reliability.

- Mean Absolute Error (MAE) values in prediction for $\mathrm{CO}$ and $\mathrm{NO}_{2}$ vary from $14 \%$ to $25 \%$ approximately and are reasonable considering the fact that the significant $\mathrm{CO}$ and $\mathrm{NO}_{2}$ emissions are from sources including industries, power plants and others in addition to mobile or transportation factors, and those factors are not specifically addressed in this study.

- Mean Absolute Error (MAE) values are large when the ANN is either under-trained or over-trained but no clear-cut rules emerge to define the best architectural configuration of ANN structure. As in other application fields where ANN modeling is successful, it is through a trial and error procedure that model compononts are identified. In a similar way, it is important that the ANN should be neither under-trained nor over-trained. 
- The Mcan Absolute Error (MAE) and Root Mean Square Error (RMSE) are less in the case of ANN, relative to the multiple regression (MR) model, indicating the robustness and accuracy of ANN over MR models.

- The measured level of $\mathrm{CO}$ pollution in ambient air decreases as ITS applications intensity or density increases. ITS helps in reducing the CO pollution level.

- Increment in ITS intensity in an area increases the concentration of $\mathrm{NO}_{2}$ in ambient air. The overall effect of a combined induced demand plus increased emissions (due to average speed increase) minus the lower congestion/delay is an increase of ambient $\mathrm{NO}_{2}$ concentrations (though small, however an increase). It may lead to the serious implications with regard to the nonattainment areas.

- MAE values in prediction for $\mathrm{O}_{3}$ vary from $5.6 \%$ to $9.1 \%$, which is far below than that of $\mathrm{CO}$ and $\mathrm{NO}_{2}$, indicating the close association of $\mathrm{O}_{3}$ (ground level) with the transportation parameters and measurable variables as compared with $\mathrm{CO}$ and $\mathrm{NO}_{2}$.

- Concentration of ground level $\mathrm{O}_{3}$ decreases with increase in the intensity of ITS application.

\subsection{Contributions to the Sum of Knowledge}

Intelligent Transportation Systems (ITS) - the application of communications and information technology to surface transportation systems - has the potential to improve transportation in several ways, from safety to emissions reductions to travel time and reliability. ITS have become a worldwide technology, and many US cities are currently 
deploying ITS, from individual technologies to entire ITS Architectures. While there is a growing number of studies evaluating the air quality benefits of ITS, there are important restrictions on the degree to which the results of these studies can be used to support planning of ITS in US cities and other nations. First, the challenges involved in modeling ITS air quality benefits mean that they normally focus on only one or two ITS technologies at a time. Second, air quality and mobility conditions diverge greatly across cities, meaning that air quality outcomes will also contrast widely. Third, present emission models are not able to take into account the effect of ITS on emission, especially the "induced demand" and the "effect on emissions with the change of average speed due to ITS". Finally, since the general public is more concerned with the ambient air quality (AAQ) rather than emissions, no models are available which could directly relate ITS to AAQ.

To date, there have been no efforts to address these issues in totality. This research provides significant contributions to the sum of knowledge in the field as follows:

- Provides an innovative method to quantify the effect of ITS on ambient air quality.

- Provides a new approach to highlight the effect of ITS on ambient air quality in contrast to the earlier research which focused more on emissions.

- Also addresses the effect of "induced demand" due to ITS on air quality.

- Neural networks, with their remarkable ability to derive meaning from complicated or imprecise data, has been used in this research for the first time to extract patterns and detect trends between ITS and air quality that 
are too complex to be noticed by either humans or other computer techniques.

- Multiple linear regression modeling was also used in conjunction with ANN to highlight the supremacy ANN modeling over other modeling techniques.

\subsection{Recommendations for future research}

Results of this study provide some conclusions that illustrate contrasts with some typical or prevailing notions. The following recommendations are therefore made to address the future research needs that may enhance the knowledge base in this field and improve model performance.

- Include more input variables which may affect the air quality (e.g., contribution of industry to air pollution) to improve accuracy in quantifying the effects of ITS on ambient air quality.

- This research shows interesting results regarding the variability of $\mathrm{NO}_{2}$ concentrations in ambient air with the intensity of application of ITS. Either the concentrations are increasing or remaining unaffected with ITS. This in turn indicates that ITS intensity leads to increasing or no affect on the concentration of $\mathrm{NO}_{2}$ in ambient air. This result may be an important observation since it may indicate conformity challenges for the entire transportation program - not just the ITS projects. A sound scientific study is therefore recommended for further investigation of this issuc.

- A thorough research study is also recommended for the investigation of the effect of increase in the average speed of motor vehicles on emissions since 
the change of emission rates with the average speed does not seem to be uniform with the different pollutants. 


\section{REFERENCES}

Boselly, E. S. (2001). Benefit/Cost Study of RWIS and Anti-icing Technologies: Final

Report. Published By: Transportation Research Board. NCHRP Report No. 20-7(117).

Bose, A. and loannou, P. (2001). Evaluation of the Environmental Effects of Intelligent Cruise Control (ICC) Vehicles. Published By: Paper presented at the 80th Annual Transportation Research Board Meeting. Washington, District of Columbia.

Bunting, A. (1997). Tracking Trucks. Published By: ITS: Intelligent Transport Systems Source Date: May/June 1997.

Burbank, C. J. (1995). ITS and the Environment.

$<$ http://www.tfhrc.gov/pubrds/spring95/p95sp9.htm> Dated: 1/22/2007.

Chao, L. and Skibniewski, M. J. (1998). Neural Networks for Evaluating Construction Technology. Artificial Neural Networks for Civil Engineers: Advanced Features and Applications. ASCE, Reston, VA, USA.

Commute Alternatives Systems Handbook (1996). Prepared by the Center for Urban Transportation Research, College of Engineering, and University of South Florida for FDOT.

Howard, D., Beale, M., and Hagan, M. (2006). NN tool box. MATLAB, The Mathworks. 2006.

Dion, Francois, et al (2002). Evaluation of Transit Signal Priority Benefits Along A Fixed-Time Signalized Arterial. Paper presented at the 81 st Annual Transportation Research Board Meeting. Washington, District of Columbia.

Dodder R. S. (2005). A System Framework for Assessing Air Quality Impacts of ITS: Application to Mexico City. MIT, Cambridge, Massachusetts, USA.

Draper, N.R. and Smith, H (1998). Applied Regression Analysis. Wiley Series in Probability and Statistics.

EPA (2008). Environmental Protection Agency. <http://www.cpa.gov/> Dated: $07 / 28 / 2008$.

EPA (2008). Clean Air Act. <http://www.epa.gov/air/caa/ > Dated: 07/24/2008. 
EPA (2008). History of Clean Air Act. <http://www.epa.gov/air/caa/caa_history.html> Dated: $07 / 24 / 2008$.

FHWA, US DOT (1996). Innovations in Transportation and Air Quality: Twelve Exemplary Projects. Published By: Federal Highway Administration, U.S. DOT.

FHWA (2008). Federal Highway Administration (FHWA). < http://www.fhwa.dot.gov/> Dated: 08/02/2008.

Fraser, N. (2008). Neural Networks. <http://ncil.fraser.name/> Dated: 08/02/2008.

Gianguido, S. and Mussone, L. (1999). Evaluation Framework and Assessment of Urban Drive Control Applications. Published By: Paper presented at the 6th World Congress Conference on ITS. Toronto, Canada. Source Date: 8-12 November 1999.

Gillen et al (1999). Assessing the Benefits and Costs of ITS Projects: Volume 2 An Application to Electronic Toll Collection. Published By: California PATH Program, University of California. Report No. UCB-ITS-PRR-99-10.

Giri, V. (2006). Presentation of Venkitila Giri for M.S. Thesis at Civil Engineering Department ,IIT, Roorkee, India.

Greenough and Kelman (1999). ITS Technology Meeting Municipal Needs - The Toronto Experience. Published By: Paper presented at the 6th World Congress Conference on ITS. Toronto, Canada. Source Date: 8-12 November 1999.

Guin, Angshuman, et al (2006). Benefits Analysis for the Georgia Department of Transportation NaviGAtor Program: Final Report. Georgia DOT.

Halkias, J. and Schauer, Michael (2004). Michigan retiming 640 traffic signals, Public Roads Journal, U.S. DOT.

Haan, C. T. (1977). Statistical Methods in Hydrology by CT Haan (1977). ISBN: 081381510X. Iowa State Publishers.

Harris, DMJM (2003). Syracuse Signal Interconnect Project: Before and After Analysis: Final Report. New York State DOT.

Highway Statistics (2005). Providing information to address major transportation issues facing the Nation. < http://www.fhwa.dot.gov/policy/ohim/hs05/index.htm> Dated: $01 / 23 / 2008$.

ITS America (2008). Intelligent Transportation Society of America. < http://www.itsa.org/> Dated: 07/30/2008.

ITS Australia (2008). Intelligent Transport Systems (ITS) Australia. $<$ http://www.itsaustralia.com.au/kmxserver3/> Dated: 07/31/2008. 
ITS Benefits (1997). Continuing Successes and Operational Test Results. USDOT, FHWA, Wasington, D. C.

ITS Europe (2.008). Intelligent Transport Systems and Services (ITS) Europe. $<$ http://www.ertico.com/> Dated: 07/31/2008.

ITS UK (2008). Intelligent Transport Systems (ITS) United Kingdom, $<$ http://www.itsuk.org.uk/> Dated: 07/31/2008.

Jeannotte, Krista (2001). Evaluation of the Advanced Regional Traffic Interactive Management and Information System (ARTIMIS). Paper presented at the 11 th Annual ITS America Meeting. Miami, Florida

Jensen, M., et al (2000). Vehicle emissions reduction by traveler information system in Seattle, Washington. Federal Highway Administration, U.S. DOT, Report No. FHWAOP-00-019.

Kandel E.R., Schwartz, J.H., Jessell, T.M. (2000). Principles of Neural Science (pp. 3673), 4th ed., McGraw-Hill, New York.

Klondzinski, Al-Deek, and Radwan (1998). Impacts of Electronic Toll Collection on Vehicle Emissions. Published By: Paper presented at the 77th Annual Transportation Rescarch Board Meeting. Washington, District of Columbia.

Kohavi, Ron (1995). A study of cross-validation and bootstrap for accuracy estimation and model selection. Proceedings of the Fourteenth International Joint Conference on Artificial Intelligence 2 (12): 1137-1143.(Morgan Kaufmann, San Mateo).

Koziol et al (1999). Evaluation of Intelligent Cruise Control System. Volume I - Study Results. Published by: Federal Highway Administration, U.S. DOT. Prepared by: Volpe for the U.S. DOT. Report No. DOT-VNTSC-NHTSA-98-3.

Language Center, AIT (2008). Writing up Research Method and Research Design. Language Center, Asian Institute of Technology, Bangkok. < http://www.languages.ait.ac.th/el21 meth.htm> Dated 07/25/2008.

Lehtonen, M. and Kulmala, R. (2002). The Benefits of a Pilot Implementation of Public Transport Signal Priorities and Real-Time Passenger Information. Published By: Paper presented at the 81 st Annual Transportation Research Board Meeting. Washington, District of Columbia.

Lingireddy and Ormsbee (1998). Neural Networks in Optimal Calibration of Water Distribution Systems. Artificial Neural Networks for Civil Engineers: Advanced Features and Applications. ASCE, Reston, VA, USA. 
Los Angeles DOT (1994). Automated Traffic Surveillance and Control (ATSAC) Evaluation Study, Published By: Los Angeles DOT.

MATLAB, 7.0 (2004). The Mathworks, 2004.

Mehta, T., Mahmassani, H. S., and Bhat, C. (2001).Methodologies for Evaluating Environmental Benefits of ITS. Texas DOT Report No. 0-4197.

MeGurrin, M. F. and Shank D. E. (1997). ITS versus New Roads: A Study of Cost Effectiveness. ITS World, July/August 1997.

Pao, Y. H. (1989), Adaptive Pattern Recognition and Neural Networks, AddisonWcsley, 1989.

RITA, (2008). Research and Innovative Technology Administration. $<$ http://www.rita.dot.gov/> Dated: 30/07/2008.

Rumelhart, D., Hinton, G., and Williams, R. (1986). Learning Internal Representation by Error Propagation. The MIT Press.

Saka, Anthony and Dennis Agboh (2002). Assessment of the Impact of Electronic Toll Collection on Mobile Emissions in the Baltimore Metropolitan Area. Paper presented at the 81 st Transportation Research Board Annual Meeting. Washington, District of Columbia.

Sapsford, R. and Jupp, V. (1996). Data Collection and Analysis. Sage Publications: London.

Silvert, W (2001). Modeling as a Discipline. Int. J. General Systems Vol. 30(3), pp. 261. Publisher: Taylor \& Francis.

Sola, J.; Sevilla, J (1997). Importance of input data normalization for the application ofneural networks to complex industrial problems. Nuclear Science, IEEE Transactions on Volume 44, Issue 3, Jun 1997 Page(s):1464 - 1468.

Srouds, J. M. (1994). Conformity and the New Transportation Covenant. Transportation Planning and Air Quality II.

Stephen, H. and McCollough, C. B. (1996). How to save \$4.2 Million a Year. ITS International Newsletter.

Tech Environmental, Inc (1993). Air Quality Benefit Study of the SmarTraveler Advanced Traveler Information Service. Tech Environmental, Inc.

Traffic Technology (1995). SCOOT in Toronto. Traffic Technology. 
Transport for London (2007). Congestion charging in London resulted in pollutant emission reductions. URL: http://www.tfl.gov.uk/assets/downloads/fifth-annual-impactsmonitoring-report-2007-07-07.pdf

Transport Research Laboratory (1999). Monitoring and evaluation of a public transport priority scheme in Southampton. Southampton University and the University of Portsmouth Transport Research Laboratory for the Hampshire County Council. Report No. TRL413.

Wilbur Smith Associates (2001). Operational and Traffic Benefits of E-ZPass to the New Jersey Turnpike. New Jersey Turnpike Authority.

Wilkie, D. (1980). Pictorial Representation of Kendall's, Rank Correlation Coefficient. Teaching Statistics 2, pp. 76-78.

Wunderlich, K., Bunch J., and Larkin J. (1999). ITS Impacts Assessment for Seattle MMDI Evaluation: Modeling Methodology and Results. Published By: Federal Highway Administration, U.S. DOT. Prepared by: Mitretek for the U.S. DOT. Source Date: September 1999. EDL Number 11323.

White et al (2000). Traffic Signal Optimization for Tysons Corner Network Volume I. Evaluation and Summary. Published By: Virginia DOT. Report No. TPE.R7D.03.08.00.

Zimmerman, C. (2000). Phoenix Metropolitan Model Deployment Initiative Evaluation Report. Published By: Federal Highway Administration, U.S. DOT. Prepared by Battelle for the U.S. DOT. Report No. FHWA-OP-00-015. 


\begin{tabular}{|c|c|c|c|c|c|}
\hline FEDERAL-AID URBANIZED AREA & Y1 & $\mathrm{Y} 2$ & Y3 & $X 1 * 1000$ & $\mathrm{X} 2$ \\
\hline New York-Newark (2000) & 0.96 & 0.0274 & 0.0463 & 17089 & 37623 \\
\hline 2002 & 0.65 & 0.0249 & 0.0549 & 17307 & 37854 \\
\hline 2004 & 0.59 & 0.0226 & 0.0475 & 17759 & 42033 \\
\hline 2005 & 0.49 & 0.0231 & 0.0502 & 17773 & 42095 \\
\hline Los Angeles-Long Beach-Santa Ana & 0.77 & 0.04 & 0.0488 & 12384 & 26949 \\
\hline 2002 & 0.81 & 0.0333 & 0.0516 & 12365 & 26329 \\
\hline 2004 & 0.53 & 0.0239 & 0.0515 & 12534 & 26284 \\
\hline 2005 & 0.56 & 0.0205 & 0.0486 & 12149 & 24726 \\
\hline Chicago (2000) & 0.71 & 0.0221 & 0.0436 & 7702 & 23764 \\
\hline 2002 & 0.644 & 0.023 & 0.0511 & 7702 & 23832 \\
\hline 2004 & 0.512 & 0.0205 & 0.045 & 7702 & 23885 \\
\hline 2005 & 0.548 & 0.0199 & 0.0527 & 7702 & 24523 \\
\hline Miami (2000) & 0.7 & 0.0097 & 0.0458 & 2270 & 5607 \\
\hline 2002 & 0.67 & 0.0097 & 0.0409 & 5021 & 15436 \\
\hline 2004 & 0.69 & 0.0084 & 0.0404 & 5270 & 16762 \\
\hline 2005 & 0.51 & 0.0084 & 0.0418 & 5331 & 17167 \\
\hline Philadelphia (2000) & 0.46 & 0.0176 & 0.052 & 4068 & 13417 \\
\hline 2002 & 0.38 & 0.016 & 0.057 & 4813 & 15743 \\
\hline 2004 & 0.35 & 0.0152 & 0.0499 & 5282 & 19029 \\
\hline 2005 & 0.36 & 0.0157 & 0.0544 & 5296 & 19078 \\
\hline Washington (2000) & 1.07 & 0.019 & 0.0471 & 3617 & 10329 \\
\hline 2002 & 0.98 & 0.02 & 0.0561 & 3807 & 10561 \\
\hline 2004 & 0.86 & 0.019 & 0.0499 & 4206 & 11438 \\
\hline 2005 & 0.86 & 0.018 & 0.0521 & 4251 & 11543 \\
\hline Atlanta $(2000)$ & 0.81 & 0.0135 & 0.0606 & 2997 & 13145 \\
\hline 2002 & 0.39 & 0.0116 & 0.0549 & 2873 & 13438 \\
\hline 2004 & 0.42 & 0.0105 & 0.0543 & 3988 & 19080 \\
\hline 2005 & 0.48 & 0.0105 & 0.0544 & 4172 & 19504 \\
\hline Boston (2000) & 0.55 & 0.0179 & 0.0451 & 2917 & 10148 \\
\hline 2002 & 0.44 & 0.017 & 0.0525 & 3854 & 13809 \\
\hline 2004 & 0.39 & 0.0144 & 0.0483 & 3977 & 16197 \\
\hline 2005 & 0.4 & 0.0146 & 0.0518 & 4077 & 16775 \\
\hline Detroit (2000) & 0.47 & 0.0215 & 0.0492 & 3836 & 13808 \\
\hline 2002 & 0.37 & 0.0193 & 0.0558 & 3835 & 13755 \\
\hline 2004 & 0.37 & 0.0159 & 0.0486 & 3930 & 14688 \\
\hline 2005 & 0.35 & 0.0174 & 0.056 & 3931 & 14638 \\
\hline Dallas-Fort Worth-Arlington (2000) & 0.6 & 0.0114 & 0.0524 & 3746 & 17830 \\
\hline 2002 & 0.5 & 0.0108 & 0.0506 & 3746 & 17778 \\
\hline
\end{tabular}




\begin{tabular}{|c|c|c|c|c|c|}
\hline 2004 & 0.46 & 0.0101 & 0.0501 & 4439 & 17772 \\
\hline 2005 & 0.45 & 0.0101 & 0.0544 & 3746 & 17799 \\
\hline Phoenix (2000) & 1.36 & 0.0291 & 0.055 & 2138 & 10232 \\
\hline 2002 & 1.19 & 0.0261 & 0.0559 & 2949 & 10684 \\
\hline 2004 & 0.84 & 0.0222 & 0.0525 & 3131 & 12077 \\
\hline 2005 & 0.83 & 0.0227 & 0.0533 & 3270 & 12418 \\
\hline San Francisco-Oakland (2000) & 0.68 & 0.0177 & 0.0473 & 4022 & 9316 \\
\hline 2002 & 0.55 & 0.0166 & 0.0489 & 4120 & 9461 \\
\hline 2004 & 0.44 & 0.0143 & 0.0461 & 4133 & 9620 \\
\hline 2005 & 0.44 & 0.014 & 0.0462 & 3110 & 7162 \\
\hline Seattle $(2000)$ & 1 & 0.0208 & 0.0394 & 1994 & 7101 \\
\hline 2002 & 0.81 & 0.0189 & 0.0425 & 2746 & 10094 \\
\hline 2004 & 0.8 & 0.0179 & 0.0412 & 2964 & 10693 \\
\hline 2005 & 0.79 & 0.018 & 0.0386 & 3002 & 10784 \\
\hline San Diego (2000) & 0.73 & 0.0181 & 0.0564 & 2653 & 5965 \\
\hline 2002 & 0.69 & 0.0176 & 0.0549 & 2823 & 6331 \\
\hline 2004 & 0.53 & 0.0155 & 0.0514 & 2869 & 6751 \\
\hline 2005 & 0.48 & 0.0156 & 0.0495 & 2903 & 5077 \\
\hline Minneapolis-St. Paul (2000) & 0.64 & 0.0171 & 0.0432 & 2475 & 10919 \\
\hline 2002 & 0.46 & 0.0147 & 0.0476 & 2440 & 10980 \\
\hline 2004 & 0.37 & 0.0111 & 0.0411 & 2482 & 11021 \\
\hline 2005 & 0.37 & 0.0118 & 0.0456 & 2519 & 11057 \\
\hline Houston $(2000)$ & 0.58 & 0.0129 & 0.054 & 2487 & 15251 \\
\hline 2002 & 0.48 & 0.0127 & 0.0517 & 2487 & 15572 \\
\hline 2004 & 0.44 & 0.0118 & 0.0512 & 2694 & 15555 \\
\hline 2005 & 0.47 & 0.0114 & 0.0541 & 2487 & 15556 \\
\hline Tampa-St. Petersburg & 0.67 & 0.0108 & 0.0532 & 1953 & 7539 \\
\hline 2002 & 0.61 & 0.0096 & 0.0452 & 2023 & 9852 \\
\hline 2004 & 0.65 & 0.0086 & 0.0472 & 2214 & 9727 \\
\hline 2005 & 0.56 & 0.008 & 0.0481 & 2251 & 9466 \\
\hline Baltimore & 0.55 & 0.0207 & 0.0556 & 2107 & 6608 \\
\hline 2002 & 0.56 & 0.0191 & 0.0643 & 2295 & 7060 \\
\hline 2004 & 0.57 & 0.0167 & 0.0535 & 2139 & 7076 \\
\hline 2005 & 0.47 & 0.0169 & 0.0555 & 2149 & 7101 \\
\hline St. Louis & 0.69 & 0.0125 & 0.0572 & 2044 & 8064 \\
\hline 2002 & 0.59 & 0.0121 & 0.0552 & 2067 & 9123 \\
\hline 2004 & 0.45 & 0.012 & 0.049 & 2092 & 9963 \\
\hline 2005 & 0.46 & 0.0115 & 0.0572 & 2106 & 10024 \\
\hline Denver-Aurora & 0.86 & 0.0368 & 0.0583 & 1993 & 7007 \\
\hline 2002 & 0.73 & 0.0354 & 0.0594 & 1989 & 7261 \\
\hline 2004 & 0.61 & 0.0272 & 0.0536 & 2067 & 7999 \\
\hline 2005 & 0.55 & 0.0276 & 0.0571 & 2092 & 8197 \\
\hline
\end{tabular}




\begin{tabular}{|c|c|c|c|c|c|}
\hline Pittsburgh & 0.52 & 0.019 & 0.0514 & 1569 & 8441 \\
\hline 2002 & 0.35 & 0.0171 & 0.0587 & 1569 & 8508 \\
\hline 2004 & 0.43 & 0.016 & 0.0497 & 1785 & 9238 \\
\hline 2005 & 0.37 & 0.0154 & 0.0534 & 1769 & 9234 \\
\hline Cleveland & 0.56 & 0.021 & 0.0543 & 1783 & 5530 \\
\hline 2002 & 0.51 & 0.0203 & 0.0587 & 1785 & 6975 \\
\hline 2004 & 0.51 & 0.0192 & 0.0497 & 1782 & 7219 \\
\hline 2005 & 0.49 & 0.0196 & 0.0562 & 1767 & 7231 \\
\hline Portland & 1.24 & 0.0122 & 0.0381 & 1338 & 5165 \\
\hline 2002 & 1.07 & 0.012 & 0.0431 & 1610 & 5750 \\
\hline 2004 & 0.93 & 0.0101 & 0.0396 & 1696 & 6610 \\
\hline 2005 & 0.85 & 0.0114 & 0.0385 & 1729 & 6891 \\
\hline Cincinnati & 0.55 & 0.0221 & 0.0547 & 1176 & 4887 \\
\hline 2002 & 0.55 & 0.0214 & 0.0597 & 1327 & 6048 \\
\hline 2004 & 0.49 & 0.0187 & 0.0504 & 1612 & 6563 \\
\hline 2005 & 0.47 & 0.0205 & 0.0564 & 1619 & 6592 \\
\hline Kansas City & 0.67 & 0.0093 & 0.0572 & 1422 & 7545 \\
\hline 2002 & 0.47 & 0.0088 & 0.0536 & 1474 & 7896 \\
\hline 2004 & 0.42 & 0.0086 & 0.0481 & 1447 & 8093 \\
\hline 2005 & 0.46 & 0.0092 & 0.0566 & 1454 & 8204 \\
\hline Milwaukee & 0.37 & 0.0165 & 0.0489 & 1532 & 5095 \\
\hline 2002 & 0.45 & 0.0171 & 0.0536 & 1443 & 5082 \\
\hline 2004 & 0.42 & 0.0112 & 0.0462 & 1378 & 6069 \\
\hline 2005 & 0.37 & 0.0124 & 0.0519 & 1399 & 6096 \\
\hline Orlando & 0.67 & 0.0111 & 0.0483 & 1160 & 3610 \\
\hline 2002 & 0.6 & 0.0088 & 0.0427 & 1235 & 6039 \\
\hline 2004 & 0.61 & 0.0079 & 0.0444 & 1300 & 5305 \\
\hline 2005 & 0.56 & 0.0065 & 0.0446 & 1335 & 5373 \\
\hline Las Vegas & 0.91 & 0.0157 & 0.0506 & 1256 & 2963 \\
\hline 2002 & 0.83 & 0.0177 & 0.0515 & 1456 & 3206 \\
\hline 2004 & 0.76 & 0.0174 & 0.0504 & 876 & 3237 \\
\hline 2005 & 0.77 & 0.0169 & 0.0508 & 1256 & 3513 \\
\hline Providence & 0.98 & 0.02 & 0.0495 & 907 & 4399 \\
\hline 2002 & 0.78 & 0.0181 & 0.058 & 1233 & 5528 \\
\hline 2004 & 0.61 & 0.0178 & 0.0518 & 1246 & 6261 \\
\hline 2005 & 0.61 & 0.0173 & 0.0528 & 1242 & 6294 \\
\hline Columbus & 0.52 & & 0.0538 & 940 & 3426 \\
\hline 2002 & 0.5 & & 0.0593 & 961 & 4747 \\
\hline 2004 & 0.47 & 0.0159 & 0.0502 & 1195 & 5250 \\
\hline 2005 & 0.39 & 0.0168 & 0.0557 & 1197 & 5301 \\
\hline San Antonio & 0.6 & 0.0108 & 0.0508 & 1143 & 5002 \\
\hline 2002 & 0.49 & 0.0104 & 0.0523 & 1259 & 5084 \\
\hline
\end{tabular}




\begin{tabular}{|c|c|c|c|c|c|}
\hline 2004 & 0.55 & 0.01 & 0.0495 & 1359 & 5095 \\
\hline 2005 & 0.44 & 0.01 & 0.0539 & 1143 & 5110 \\
\hline Buffalo & 0.51 & 0.0246 & 0.0462 & 1112 & 3985 \\
\hline 2002 & 0.41 & 0.0197 & 0.0523 & 1123 & 4049 \\
\hline 2004 & 0.37 & 0.0167 & 0.0468 & 1123 & 4025 \\
\hline 2005 & 0.37 & 0.0172 & 0.0495 & 1123 & 4025 \\
\hline New Orleans & 0.7 & 0.0109 & 0.0499 & 1065 & 3290 \\
\hline 2002 & 0.63 & 0.0098 & 0.0437 & 1065 & 3292 \\
\hline 2004 & 0.68 & 0.0095 & 0.0449 & 1009 & 3279 \\
\hline 2005 & 0.58 & 0.0095 & 0.0492 & 1009 & 3285 \\
\hline Jacksonville & 0.61 & 0.0104 & 0.0591 & 869 & 3664 \\
\hline 2002 & 0.54 & 0.0094 & 0.0462 & 906 & 4769 \\
\hline 2004 & 0.5 & 0.0082 & 0.0492 & 965 & 5155 \\
\hline 2005 & 0.48 & 0.0077 & 0.0495 & 992 & 5067 \\
\hline Nashville & 0.69 & 0.0184 & 0.0591 & 605 & 2960 \\
\hline 2002 & 0.69 & 0.0144 & 0.0536 & 659 & 2958 \\
\hline 2004 & 0.61 & 0.0117 & 0.0514 & 974 & 4641 \\
\hline 2005 & 0.58 & 0.0143 & 0.0573 & 984 & 4650 \\
\hline Salt Lake City & 1.15 & 0.0264 & 0.0618 & 830 & 3334 \\
\hline 2002 & 0.95 & 0.027 & 0.0644 & 910 & 3399 \\
\hline 2004 & 0.86 & 0.0253 & 0.0591 & 937 & 3384 \\
\hline 2005 & 0.83 & 0.0234 & 0.0629 & 970 & 3444 \\
\hline Indianapolis & 0.73 & 0.0151 & 0.0547 & 915 & 4228 \\
\hline 2002 & 0.66 & 0.0138 & 0.0621 & 915 & 4334 \\
\hline 2004 & 0.65 & 0.0111 & 0.0538 & 915 & 4438 \\
\hline 2005 & 0.55 & 0.0121 & 0.0593 & 915 & 4755 \\
\hline Richmond & 0.43 & 0.0153 & 0.0544 & 694 & 2964 \\
\hline 2002 & 0.46 & 0.0154 & 0.06 & 838 & 4346 \\
\hline 2004 & 0.39 & 0.0147 & 0.0516 & 898 & 4648 \\
\hline 2005 & 0.42 & 0.0147 & 0.0548 & 910 & 4682 \\
\hline Louisville & 0.48 & 0.0144 & 0.0557 & 823 & 3763 \\
\hline 2002 & 0.56 & 0.0127 & 0.0541 & 841 & 3747 \\
\hline 2004 & 0.65 & 0.0122 & 0.0495 & 898 & 4154 \\
\hline 2005 & 0.56 & 0.0123 & 0.0546 & 904 & 4394 \\
\hline Hartford-Middlctown & 0.82 & 0.0166 & 0.0526 & 602 & 2597 \\
\hline 2002 & 0.79 & 0.0179 & 0.0606 & 873 & 4023 \\
\hline 2004 & 0.86 & 0.0149 & 0.0526 & 885 & 4054 \\
\hline 2005 & 0.85 & 0.0155 & 0.0569 & 889 & 4062 \\
\hline Oklahoma City & 0.74 & 0.0119 & 0.0522 & 1083 & 4714 \\
\hline 2002 & 0.49 & 0.0119 & 0.0509 & 1083 & 4711 \\
\hline 2004 & 0.43 & 0.0093 & 0.0492 & 834 & 4404 \\
\hline 2005 & 0.35 & 0.0103 & 0.055 & 856 & 4531 \\
\hline
\end{tabular}




\begin{tabular}{|c|c|c|c|c|c|}
\hline Charlotte & 0.66 & 0.0179 & 0.0606 & 646 & 2653 \\
\hline 2002 & 0.55 & 0.0145 & 0.0603 & 721 & 2812 \\
\hline 2004 & 0.49 & 0.0137 & 0.0495 & 743 & 2950 \\
\hline 2005 & 0.49 & 0.0127 & 0.055 & 855 & 3737 \\
\hline Tucson & 0.77 & 0.0206 & 0.0534 & 619 & 2225 \\
\hline 2002 & 0.7 & 0.0192 & 0.0535 & 709 & 3095 \\
\hline 2004 & 0.58 & 0.0183 & 0.0498 & 731 & 3786 \\
\hline 2005 & 0.58 & 0.0178 & 0.051 & 749 & 3715 \\
\hline Birmingham & 0.98 & 0.0107 & 0.0579 & 667 & 4595 \\
\hline 2002 & 0.72 & 0.0061 & 0.0517 & 664 & 4613 \\
\hline 2004 & 0.58 & 0.0041 & 0.0498 & 676 & 4773 \\
\hline 2005 & 0.7 & 0.0052 & 0.0519 & 680 & 4780 \\
\hline El Paso & 0.68 & 0.011 & 0.0519 & 649 & 2211 \\
\hline 2002 & 0.54 & 0.01 & 0.0525 & 657 & 2225 \\
\hline 2004 & 0.53 & 0.0096 & 0.0501 & 637 & 2235 \\
\hline 2005 & 0.47 & 0.0094 & 0.0533 & 656 & 2235 \\
\hline Honolulu & 0.73 & 0.005 & 0.0242 & 694 & 1068 \\
\hline 2002 & 0.7 & 0.005 & 0.0267 & 694 & 1081 \\
\hline 2004 & 0.68 & 0.004 & 0.0249 & 648 & 1090 \\
\hline 2005 & 0.57 & 0.004 & 0.0263 & 648 & 1092 \\
\hline Fresno & 0.65 & 0.0153 & 0.0447 & 555 & 2191 \\
\hline 2002 & 0.58 & 0.0147 & 0.0478 & 586 & 2191 \\
\hline 2004 & 0.44 & 0.0129 & 0.0453 & 603 & 2257 \\
\hline 2005 & 0.45 & 0.0126 & 0.044 & 616 & 2291 \\
\hline Allentown-Bethlehem & 0.46 & 0.0157 & 0.0516 & 447 & 1872 \\
\hline 2002 & 0.35 & 0.0143 & 0.0583 & 448 & 1979 \\
\hline 2004 & 0.29 & 0.0136 & 0.0508 & 599 & 3032 \\
\hline 2005 & 0.35 & 0.0123 & 0.055 & 607 & 3039 \\
\hline Grand Rapids & 0.41 & 0.0216 & 0.0502 & 530 & 2273 \\
\hline 2002 & 0.38 & 0.0193 & 0.0581 & 529 & 2260 \\
\hline 2004 & 0.39 & 0.0159 & 0.0508 & 591 & 2722 \\
\hline 2005 & 0.36 & 0.0174 & 0.0565 & 595 & 2672 \\
\hline Springfield & 0.68 & 0.0162 & 0.0482 & 608 & 2928 \\
\hline 2002 & 0.79 & 0.0147 & 0.056 & 653 & 3144 \\
\hline 2004 & 0.46 & 0.0112 & 0.0525 & 589 & 3230 \\
\hline 2005 & 0.41 & 0.011 & 0.0558 & 587 & 3253 \\
\hline Tulsa & 0.71 & 0.0153 & 0.0525 & 803 & 2761 \\
\hline 2002 & 0.7 & 0.0105 & 0.051 & 803 & 2771 \\
\hline 2004 & 0.4 & 0.0054 & 0.0478 & 559 & 3323 \\
\hline 2005 & 0.49 & 0.0109 & 0.0552 & 575 & 3325 \\
\hline Albuquerque & 0.65 & 0.0098 & 0.0534 & 427 & 1949 \\
\hline 2002 & 0.51 & 0.0108 & 0.0536 & 529 & 1956 \\
\hline
\end{tabular}




\begin{tabular}{|c|c|c|c|c|c|}
\hline 2004 & 0.42 & 0.0113 & 0.0497 & 543 & 2515 \\
\hline 2005 & 0.41 & 0.0106 & 0.0504 & 573 & 2537 \\
\hline Omaha & 0.56 & & 0.0447 & 648 & 2475 \\
\hline 2002 & 0.54 & & 0.0429 & 625 & 2483 \\
\hline 2004 & 0.52 & & 0.0453 & 571 & 2481 \\
\hline 2005 & 0.5 & & 0.0468 & 571 & 2500 \\
\hline Albany & 0.48 & 0.0227 & 0.0489 & 519 & 2663 \\
\hline 2002 & 0.39 & 0.0182 & 0.0551 & 524 & 2607 \\
\hline 2004 & 0.35 & 0.0155 & 0.0483 & 524 & 2592 \\
\hline 2005 & 0.34 & 0.0162 & 0.0505 & 524 & 2592 \\
\hline Tolcdo & 0.55 & & 0.0553 & 500 & 2121 \\
\hline 2002 & 0.58 & & 0.0593 & 499 & 2267 \\
\hline 2004 & 0.46 & & 0.0493 & 518 & 2439 \\
\hline 2005 & 0.49 & & 0.0552 & 518 & 2432 \\
\hline Knoxville & 0.57 & 0.0089 & 0.0615 & 325 & 1997 \\
\hline 2002 & 0.66 & 0.0044 & 0.0551 & 363 & 2048 \\
\hline 2004 & & & 0.0545 & 483 & 2710 \\
\hline 2005 & & 0.0036 & 0.059 & 483 & 2965 \\
\hline Baton Rouge & 0.74 & 0.0106 & 0.0508 & 375 & 1823 \\
\hline 2002 & 0.7 & 0.0098 & 0.0441 & 375 & 1827 \\
\hline 2004 & 0.54 & 0.0095 & 0.046 & 479 & 2136 \\
\hline 2005 & 0.43 & 0.0102 & 0.0503 & 479 & 2159 \\
\hline Bakersfield & 0.6 & 0.018 & 0.0534 & 404 & 1377 \\
\hline 2002 & 0.5 & 0.0166 & 0.053 & 425 & 1355 \\
\hline 2004 & 0.4 & 0.0141 & 0.0492 & 448 & 1435 \\
\hline 2005 & 0.37 & 0.0135 & 0.0496 & 469 & 1450 \\
\hline Youngstown & 0.5 & & 0.055 & 379 & 1821 \\
\hline 2002 & 0.55 & & 0.0604 & 423 & 2281 \\
\hline 2004 & 0.46 & & 0.052 & 441 & 2598 \\
\hline 2005 & 0.42 & & 0.0578 & 444 & 2598 \\
\hline
\end{tabular}




\begin{tabular}{|c|c|c|c|c|c|c|c|c|}
\hline$\times 3 * 1000$ & $\mathrm{X} 4$ & $X 5^{*} 100$ & $x 6$ & X7 & $\mathrm{X} 8$ & $\mathrm{X} 9$ & $\times 10$ & X11 \\
\hline 263905 & 3962 & 101290 & 1130 & 89639 & 27 & 22 & 24 & 51 \\
\hline 274767 & 3962 & 105197 & 1144 & 91950 & 37 & 31 & 29 & 63 \\
\hline 297930 & 4778 & 115408 & 1220 & 94596 & 13 & 23 & 59 & 32 \\
\hline 299706 & 4778 & 117154 & 1221 & 95949 & 14 & 6 & 53 & 16 \\
\hline 280793 & 2231 & 126498 & 652 & 193875 & 2 & 0 & 3 & 15 \\
\hline 292516 & 2231 & 135339 & 705 & 191970 & 4 & 0 & 7 & 33 \\
\hline 293758 & 2231 & 139274 & 704 & 197832 & 8 & 31 & 33 & 47 \\
\hline 279040 & 1948 & 133081 & 673 & 197743 & 11 & 31 & 36 & 47 \\
\hline 158240 & 2730 & 48276 & 477 & 101167 & 17 & 28 & 14 & 45 \\
\hline 165494 & 2730 & 51424 & 478 & 107682 & 18 & 28 & 14 & 45 \\
\hline 170869 & 2730 & 53942 & 478 & 112849 & 13 & 46 & 25 & 39 \\
\hline 170933 & 3502 & 54398 & 485 & 112162 & 22 & 16 & 35 & 48 \\
\hline 43577 & 353 & 13584 & 120 & 112782 & 13 & 0 & 7 & 96 \\
\hline 120131 & 1590 & 35699 & 478 & 113013 & 39 & 7 & 10 & 100 \\
\hline 131852 & 1499 & 38322 & 332 & 115428 & 39 & 7 & 10 & 100 \\
\hline 132934 & 1499 & 39468 & 329 & 119963 & 39 & 25 & 28 & 100 \\
\hline 77005 & 1347 & 24483 & 347 & 70457 & 49 & 15 & 17 & 65 \\
\hline 93445 & 1590 & 30772 & 407 & 75628 & 59 & 24 & 22 & 51 \\
\hline 105839 & 2257 & 34442 & 455 & 75696 & 31 & 16 & 22 & 48 \\
\hline 106558 & 2257 & 35324 & 468 & 75478 & 45 & 16 & 35 & 50 \\
\hline 82959 & 999 & 34533 & 306 & 112852 & 27 & 19 & 34 & 81 \\
\hline 86519 & 999 & 36201 & 306 & 118255 & 33 & 19 & 55 & 46 \\
\hline 94429 & 1305 & 38197 & 325 & 117529 & 41 & 19 & 54 & 46 \\
\hline 97009 & 1305 & 38582 & 330 & 116915 & 48 & 19 & 61 & 52 \\
\hline 100693 & 1757 & 42488 & 306 & 138701 & 0 & 11 & 11 & 73 \\
\hline 101402 & 1757 & 43388 & 300 & 144675 & 0 & 26 & 26 & 88 \\
\hline 125528 & 3027 & 49489 & 358 & 138237 & 0 & 45 & 45 & 88 \\
\hline 128353 & 3027 & 49192 & 360 & 136644 & 0 & 51 & 51 & 88 \\
\hline 59361 & 1138 & 22890 & 211 & 108468 & 5 & 3 & 20 & 85 \\
\hline 80693 & 1695 & 33130 & 342 & 96856 & 5 & 3 & 20 & 85 \\
\hline 90971 & 2104 & 38722 & 422 & 91758 & 5 & 0 & 20 & 85 \\
\hline 94248 & 2241 & 40873 & 446 & 91643 & 5 & 0 & 20 & 81 \\
\hline 92359 & 1304 & 31125 & 283 & 109882 & 0 & 39 & 39 & 9 \\
\hline 96388 & 1304 & 31613 & 280 & 112987 & 0 & 39 & 39 & 39 \\
\hline 102234 & 1439 & 33459 & 304 & 110061 & 4 & 39 & 39 & 39 \\
\hline 104126 & 1439 & 33045 & 303 & 109059 & 4 & 39 & 39 & 39 \\
\hline 116548 & 1712 & 49197 & 594 & 82872 & 0 & 5 & 11 & 50 \\
\hline 107298 & 1727 & 50965 & 518 & 98431 & 0 & 8 & 14 & 50 \\
\hline
\end{tabular}




\begin{tabular}{|c|c|c|c|c|c|c|c|c|}
\hline 113395 & 2385 & 52162 & 520 & 100312 & 0 & 34 & 15 & 56 \\
\hline 119648 & 1712 & 57326 & 522 & 109819 & 0 & 34 & 16 & 56 \\
\hline 58405 & 1054 & 19424 & 163 & 118882 & 0 & 0 & 31 & 74 \\
\hline 62566 & 1054 & 22528 & 189 & 118967 & 0 & 0 & 59 & 79 \\
\hline 73269 & 1151 & 26674 & 191 & 139654 & 0 & 48 & 48 & 99 \\
\hline 77267 & 1115 & 28728 & 202 & 142219 & 0 & 43 & 43 & 99 \\
\hline 90277 & 1203 & 47982 & 330 & 145461 & 41 & 0 & 41 & 84 \\
\hline 91945 & 1203 & 48583 & 341 & 142611 & 47 & 0 & 70 & 100 \\
\hline 92009 & 1203 & 49263 & 343 & 143625 & 47 & 0 & 70 & 100 \\
\hline 73251 & 721 & 39501 & 274 & 144163 & 47 & 0 & 70 & 100 \\
\hline 51430 & 844 & 24008 & 241 & 99474 & 7 & 0 & 26 & 6 \\
\hline 67330 & 1185 & 30465 & 296 & 102960 & 24 & 0 & 44 & 68 \\
\hline 69593 & 1185 & 30418 & 321 & 94761 & 27 & 0 & 48 & 68 \\
\hline 69967 & 1185 & 30759 & 321 & 95824 & 27 & 0 & 48 & 68 \\
\hline 62809 & 733 & 33745 & 246 & 137029 & 10 & 0 & 7 & 70 \\
\hline 66391 & 733 & 33502 & 254 & 131875 & 10 & 0 & 7 & 70 \\
\hline 70102 & 733 & 38807 & 270 & 143731 & 10 & 0 & 10 & 70 \\
\hline 70408 & 953 & 39396 & 277 & 142224 & 1 & 0 & 23 & 77 \\
\hline 60720 & 1192 & 27094 & 316 & 85640 & 87 & 0 & 59 & 29 \\
\hline 62430 & 1192 & 27060 & 317 & 85335 & 87 & 0 & 64 & 49 \\
\hline 63154 & 1192 & 27398 & 317 & 86429 & 87 & 0 & 64 & 49 \\
\hline 63492 & 1192 & 28142 & 321 & 87671 & 87 & 0 & 64 & 49 \\
\hline 91883 & 1537 & 39195 & 368 & 106458 & 0 & 0 & 41 & 24 \\
\hline 97614 & 1476 & 45165 & 364 & 124109 & 12 & 0 & 52 & 24 \\
\hline 97525 & 1954 & 45631 & 378 & 120718 & 12 & 0 & 55 & 24 \\
\hline 97775 & 1537 & 46372 & 378 & 122676 & 12 & 0 & 58 & 24 \\
\hline 44473 & 650 & 8356 & 124 & 67181 & 13 & 0 & 5 & 6 \\
\hline 55540 & 1294 & 9380 & 127 & 73956 & 0 & 0 & 5 & 30 \\
\hline 62796 & 1072 & 12982 & 157 & 82690 & 0 & 0 & 5 & 31 \\
\hline 63177 & 1072 & 13074 & 160 & 81715 & 0 & 0 & 8 & 36 \\
\hline 45021 & 712 & 22659 & 278 & 81550 & 20 & 0 & 23 & 72 \\
\hline 50256 & 764 & 25428 & 287 & 88565 & 46 & 0 & 38 & 54 \\
\hline 52006 & 683 & 26336 & 288 & 91445 & 23 & 0 & 19 & 27 \\
\hline 52541 & 683 & 26454 & 287 & 92176 & 23 & 0 & 20 & 27 \\
\hline 58761 & 1124 & 25739 & 320 & 80362 & 27 & 11 & 0 & 39 \\
\hline 60292 & 1124 & 25773 & 321 & 80373 & 27 & 11 & 0 & 50 \\
\hline 64764 & 1135 & 27663 & 353 & 78365 & 27 & 11 & 12 & 53 \\
\hline 63585 & 1359 & 27130 & 352 & 77073 & 27 & 11 & 12 & 53 \\
\hline 43997 & 720 & 16904 & 209 & 81063 & 0 & 0 & 6 & 0 \\
\hline 45479 & 720 & 17079 & 208 & 82039 & 15 & 0 & 9 & 29 \\
\hline 49754 & 814 & 18615 & 258 & 72150 & 29 & 0 & 9 & 29 \\
\hline 52437 & 814 & 19897 & 259 & 76823 & 41 & 0 & 9 & 29 \\
\hline
\end{tabular}




\begin{tabular}{|c|c|c|c|c|c|c|c|}
\hline 35632 & 1086 & 11128 & 283 & 39295 & 14 & 0 & 9 \\
\hline 36154 & 1086 & 11700 & 290 & 40413 & 41 & 9 & 9 \\
\hline 38996 & 1215 & 12500 & 300 & 41666 & 41 & 9 & 17 \\
\hline 38673 & 1215 & 12330 & 301 & 40964 & 41 & 9 & 17 \\
\hline 37800 & 838 & 17284 & 227 & 76169 & 14 & 0 & 4 \\
\hline 36757 & 838 & 16782 & 227 & 73957 & 14 & 0 & 0 \\
\hline 38645 & 897 & 18374 & 244 & 75304 & 14 & 0 & 2 \\
\hline 38642 & 897 & 18150 & 244 & 74386 & 18 & 0 & 2 \\
\hline 31517 & 469 & 12595 & 137 & 91900 & 0 & 53 & 44 \\
\hline 31826 & 469 & 12901 & 137 & 94227 & 0 & 62 & 58 \\
\hline 34237 & 538 & 13098 & 145 & 90264 & 0 & 71 & 62 \\
\hline 35143 & 538 & 13621 & 151 & 90204 & 0 & 73 & 66 \\
\hline 32605 & 630 & 15744 & 176 & 89495 & 51 & 38 & 38 \\
\hline 35236 & 691 & 16128 & 180 & 89796 & 51 & 38 & 38 \\
\hline 39625 & 887 & 17790 & 209 & 85119 & 51 & 38 & 43 \\
\hline 40148 & 887 & 18560 & 209 & 88804 & 51 & 38 & 43 \\
\hline 41187 & 1036 & 19307 & 374 & 51566 & 7 & 0 & 0 \\
\hline 42262 & 1036 & 20067 & 381 & 52662 & 7 & 0 & 0 \\
\hline 41484 & 1041 & 20183 & 376 & 53678 & 11 & 26 & 26 \\
\hline 44359 & 1041 & 20674 & 384 & 53838 & 11 & 28 & 28 \\
\hline 31888 & 518 & 9701 & 111 & 87013 & 31 & 78 & 45 \\
\hline 31753 & 512 & 9304 & 111 & 83933 & 31 & 78 & 78 \\
\hline 34355 & 515 & 10943 & 144 & 75995 & 58 & 78 & 89 \\
\hline 33933 & 518 & 10746 & 141 & 76212 & 70 & 93 & 89 \\
\hline 32288 & 395 & 9532 & 156 & 60915 & 0 & 33 & 33 \\
\hline 40383 & 667 & 9934 & 162 & 61404 & 0 & 33 & 33 \\
\hline 40972 & 716 & 11768 & 177 & 66486 & 0 & 100 & 100 \\
\hline 42447 & 716 & 12468 & 167 & 74656 & 0 & 100 & 100 \\
\hline 24128 & 270 & 6848 & 77 & 88954 & 0 & 0 & 0 \\
\hline 26408 & 270 & 7985 & 83 & 96143 & 13 & 0 & 0 \\
\hline 27490 & 270 & 8277 & 83 & 99722 & 0 & 0 & 0 \\
\hline 30543 & 270 & 9228 & 86 & 107299 & 13 & 4 & 4 \\
\hline 20446 & 515 & 8465 & 120 & 70833 & 29 & 0 & 0 \\
\hline 24881 & 794 & 10588 & 157 & 67417 & 22 & 0 & 41 \\
\hline 26698 & 799 & 11415 & 182 & 62721 & 13 & 0 & 47 \\
\hline 26746 & 799 & 11505 & 182 & 63214 & 18 & 0 & 91 \\
\hline 24731 & 476 & 11895 & 149 & 80044 & 0 & 0 & 15 \\
\hline 26394 & 476 & 13354 & 150 & 88865 & 87 & 9 & 0 \\
\hline 31208 & 609 & 15043 & 164 & 91724 & 0 & 9 & 9 \\
\hline 31104 & 609 & 14960 & 164 & 91219 & 0 & 9 & 9 \\
\hline 33445 & 485 & 15775 & 211 & 74837 & 0 & 0 & 0 \\
\hline 31354 & 481 & 15908 & 212 & 75027 & 0 & 41 & 41 \\
\hline
\end{tabular}




\begin{tabular}{|c|c|c|c|c|c|c|c|c|}
\hline 32817 & 565 & 16792 & 212 & 79208 & 2 & 40 & 40 & 87 \\
\hline 33681 & 485 & 17064 & 211 & 80870 & 2 & 40 & 40 & 87 \\
\hline 21448 & 564 & 6365 & 139 & 45900 & 33 & 0 & 8 & 0 \\
\hline 21662 & 564 & 6434 & 139 & 46356 & 33 & 0 & 0 & 14 \\
\hline 22320 & 564 & 6722 & 139 & 48358 & 33 & 0 & 85 & 8 \\
\hline 22320 & 564 & 6722 & 139 & 48358 & 33 & 0 & 62 & 8 \\
\hline 15414 & 270 & 5613 & 75 & 74954 & 0 & 0 & 0 & 16 \\
\hline 15945 & 270 & 5964 & 75 & 79220 & 33 & 0 & 0 & 68 \\
\hline 15468 & 321 & 5411 & 66 & 81989 & 33 & 0 & 0 & 68 \\
\hline 15330 & 321 & 5415 & 69 & 78480 & 33 & 0 & 0 & 68 \\
\hline 24553 & 508 & 9836 & 156 & 62896 & 0 & 0 & 11 & 55 \\
\hline 28095 & 727 & 9963 & 151 & 66091 & 0 & 5 & 11 & 100 \\
\hline 32861 & 696 & 10823 & 157 & 68938 & 0 & 5 & 11 & 100 \\
\hline 33078 & 696 & 11247 & 158 & 71185 & 3 & 21 & 31 & 100 \\
\hline 22753 & 571 & 10433 & 139 & 74814 & 0 & 0 & 0 & 49 \\
\hline 23302 & 571 & 10727 & 139 & 76932 & 0 & 0 & 21 & 49 \\
\hline 31556 & 746 & 13002 & 173 & 75157 & 11 & 21 & 21 & 49 \\
\hline 31955 & 746 & 13369 & 172 & 77729 & 11 & 21 & 21 & 28 \\
\hline 20396 & 353 & 6410 & 79 & 81618 & 15 & 0 & 72 & 86 \\
\hline 22578 & 353 & 8293 & 80 & 104261 & 36 & 0 & 100 & 100 \\
\hline 21329 & 342 & 7540 & 80 & 94249 & 65 & 0 & 100 & 100 \\
\hline 21294 & 342 & 7565 & 76 & 99534 & 11 & 0 & 100 & 100 \\
\hline 29398 & 422 & 11259 & 130 & 86750 & 13 & 0 & 6 & 24 \\
\hline 30950 & 422 & 10969 & 130 & 84603 & 78 & 0 & 0 & 39 \\
\hline 30634 & 422 & 11288 & 130 & 86828 & 78 & 0 & 16 & 39 \\
\hline 30571 & 422 & 11050 & 129 & 85661 & 78 & 0 & 16 & 39 \\
\hline 16879 & 406 & 6937 & 109 & 63678 & 24 & 0 & 0 & 0 \\
\hline 24648 & 473 & 9965 & 178 & 55935 & 24 & 0 & 4 & 0 \\
\hline 25816 & 697 & 11510 & 194 & 59331 & 24 & 0 & 4 & 0 \\
\hline 26187 & 697 & 11682 & 194 & 60215 & 24 & 0 & 9 & 0 \\
\hline 22794 & 384 & 10040 & 137 & 73103 & 1 & 0 & 0 & 43 \\
\hline 22695 & 384 & 10442 & 137 & 76034 & 7 & 16 & 16 & 43 \\
\hline 24703 & 465 & 11730 & 150 & 78200 & 18 & 28 & 28 & 43 \\
\hline 24515 & 465 & 11583 & 150 & 77218 & 35 & 35 & 35 & 43 \\
\hline 16187 & 366 & 8407 & 108 & 77582 & 16 & 12 & 4 & 31 \\
\hline 21765 & 552 & 10359 & 145 & 71283 & 16 & 10 & 31 & 34 \\
\hline 22114 & 552 & 10566 & 146 & 72371 & 16 & 10 & 31 & 34 \\
\hline 22250 & 552 & 10589 & 147 & 72034 & 16 & 10 & 31 & 34 \\
\hline 25980 & 647 & 8932 & 150 & 59444 & 0 & 0 & 0 & 0 \\
\hline 27216 & 647 & 9496 & 156 & 60998 & 0 & 0 & 0 & 0 \\
\hline 26393 & 548 & 9230 & 143 & 64544 & 0 & 0 & 0 & 0 \\
\hline 27144 & 548 & 9243 & 143 & 64639 & 0 & 0 & 100 & 0 \\
\hline
\end{tabular}




\begin{tabular}{|c|c|c|c|c|c|c|c|}
\hline 19097 & 299 & 7640 & 92 & 82958 & 18 & 29 & 14 \\
\hline 19807 & 299 & 7862 & 92 & 85177 & 30 & 30 & 31 \\
\hline 20403 & 299 & 8046 & 100 & 80458 & 16 & 15 & 25 \\
\hline 29513 & 583 & 10984 & 132 & 83213 & 16 & 27 & 45 \\
\hline 13400 & 280 & 2153 & 34 & 62464 & 0 & 0 & 0 \\
\hline 14011 & 312 & 2359 & 35 & 68056 & 0 & 0 & 9 \\
\hline 17280 & 503 & 3424 & 51 & 67132 & 0 & 0 & 9 \\
\hline 18505 & 503 & 3553 & 48 & 74016 & 0 & 0 & 9 \\
\hline 23185 & 609 & 8687 & 127 & 68422 & 0 & 0 & 15 \\
\hline 23331 & 609 & 8762 & 127 & 69023 & 0 & 0 & 15 \\
\hline 24046 & 609 & 9270 & 127 & 72992 & 0 & 0 & 15 \\
\hline 24722 & 609 & 9645 & 128 & 75352 & 0 & 0 & 15 \\
\hline 12049 & 227 & 3977 & 51 & 77660 & 0 & 34 & 34 \\
\hline 11396 & 226 & 4012 & 51 & 78337 & 0 & 44 & 44 \\
\hline 12286 & 259 & 4479 & 51 & 87824 & 0 & 44 & 71 \\
\hline 12636 & 229 & 4554 & 51 & 89287 & 0 & 44 & 100 \\
\hline 11434 & 135 & 5626 & 69 & 81898 & 0 & 0 & 0 \\
\hline 11814 & 135 & 5776 & 69 & 84046 & 0 & 0 & 14 \\
\hline 12858 & 139 & 6001 & 69 & 86969 & 0 & 0 & 14 \\
\hline 13028 & 139 & 5943 & 69 & 86126 & 0 & 0 & 14 \\
\hline 11598 & 168 & 2552 & 36 & 70049 & 19 & 0 & 22 \\
\hline 12290 & 168 & 3216 & 47 & 68690 & 24 & 0 & 26 \\
\hline 12456 & 168 & 3647 & 55 & 66309 & 24 & 0 & 26 \\
\hline 12579 & 190 & 3863 & 55 & 70245 & 24 & 0 & 26 \\
\hline 9443 & 179 & 3380 & 65 & 51991 & 10 & 0 & 0 \\
\hline 9553 & 179 & 3694 & 68 & 53998 & 100 & 0 & 0 \\
\hline 13308 & 339 & 4871 & 92 & 52943 & 100 & 0 & 27 \\
\hline 13511 & 339 & 5085 & 97 & 52418 & 100 & 0 & 27 \\
\hline 12151 & 318 & 3566 & 68 & 52794 & 0 & 0 & 0 \\
\hline 12636 & 318 & 3841 & 68 & 56793 & 0 & 0 & 0 \\
\hline 15151 & 443 & 4897 & 100 & 48975 & 0 & 0 & 15 \\
\hline 15540 & 443 & 4885 & 101 & 48363 & 0 & 0 & 21 \\
\hline 13124 & 422 & 4249 & 79 & 54021 & 0 & 0 & 10 \\
\hline 14030 & 463 & 4803 & 87 & 54928 & 0 & 0 & 10 \\
\hline 14225 & 477 & 5314 & 95 & 55937 & 0 & 0 & 10 \\
\hline 14534 & 477 & 5489 & 95 & 57784 & 0 & 0 & 10 \\
\hline 18006 & 305 & 6267 & 112 & 55813 & 0 & 0 & 0 \\
\hline 18974 & 305 & 6595 & 132 & 49774 & 0 & 0 & 0 \\
\hline 20688 & 474 & 6936 & 151 & 45937 & 0 & 0 & 0 \\
\hline 20904 & 474 & 6958 & 152 & 45778 & 0 & 0 & 10 \\
\hline 12145 & 175 & 3356 & 44 & 77055 & 0 & 0 & 0 \\
\hline 12493 & 175 & 3398 & 51 & 66166 & 30 & 0 & 15 \\
\hline
\end{tabular}




$\begin{array}{lllllllll}14263 & 192 & 4516 & 64 & 70557 & 30 & 0 & 15 & 30 \\ 14534 & 269 & 4664 & 64 & 72879 & 30 & 0 & 15 & 30 \\ 12194 & 222 & 3298 & 60 & 55425 & 0 & 0 & 0 & 47 \\ 12809 & 222 & 3635 & 60 & 61098 & 0 & 0 & 0 & 63 \\ 12986 & 222 & 3529 & 56 & 63018 & 0 & 0 & 0 & 63 \\ 13225 & 222 & 3591 & 56 & 64119 & 0 & 0 & 0 & 63 \\ 13955 & 365 & 5503 & 104 & 53129 & 7 & 0 & 21 & 28 \\ 14568 & 365 & 5817 & 104 & 56168 & 24 & 0 & 28 & 28 \\ 15134 & 365 & 6214 & 104 & 59747 & 24 & 0 & 23 & 32 \\ 15134 & 365 & 6214 & 104 & 59747 & 17 & 0 & 8 & 0 \\ 11830 & 255 & 4024 & 71 & 56794 & 0 & 0 & 0 & 0 \\ 12195 & 255 & 4091 & 71 & 57599 & 0 & 0 & 0 & 0 \\ 13101 & 287 & 4154 & 72 & 57696 & 0 & 0 & 1 & 0 \\ 12950 & 287 & 4090 & 72 & 56802 & 0 & 0 & 1 & 0 \\ 11574 & 355 & 3994 & 51 & 78347 & 0 & 0 & 0 & 42 \\ 12750 & 355 & 4265 & 52 & 82032 & 0 & 0 & 0 & 61 \\ 15776 & 458 & 5028 & 63 & 79809 & 0 & 0 & 0 & 61 \\ 16430 & 458 & 5136 & 63 & 81526 & 85 & 0 & 63 & 61 \\ 8334 & 185 & 2487 & 36 & 69243 & 0 & 0 & 0 & 100 \\ 9016 & 185 & 2761 & 36 & 76898 & 0 & 0 & 0 & 0 \\ 12257 & 638 & 3630 & 52 & 69812 & 0 & 0 & 97 & 97 \\ 13093 & 638 & 4054 & 60 & 67572 & 32 & 0 & 100 & 100 \\ 7270 & 176 & 1929 & 42 & 46373 & 9 & 0 & 11 & 4 \\ 7602 & 176 & 2047 & 42 & 49173 & 17 & 0 & 11 & 0 \\ 7891 & 176 & 2034 & 42 & 48426 & 17 & 0 & 11 & 0 \\ 8351 & 226 & 2063 & 42 & 49111 & 17 & 0 & 11 & 0 \\ 7269 & 193 & 1624 & 46 & 35258 & 0 & 0 & 0 & 0 \\ 8155 & 243 & 1735 & 54 & 32327 & 0 & 0 & 0 & 0 \\ 9609 & 325 & 2462 & 82 & 30024 & 0 & 0 & 0 & 0 \\ 9629 & 325 & 2476 & 84 & 29482 & 0 & 0 & 0 & 0\end{array}$




\begin{tabular}{|c|c|c|c|c|c|c|c|c|}
\hline $\mathrm{X} 12$ & $X 13$ & X14 & X15 & X16 & X17 & X18 & $\times 19$ & $\times 20$ \\
\hline 1 & 6 & 23 & 18 & 48 & 1 & 71 & 21 & 18 \\
\hline 1 & 6 & 10 & 27 & 43 & 1 & 60 & 7 & 13 \\
\hline 3 & 9 & 30 & 29 & 45 & 3 & 76 & 7 & 34 \\
\hline 1 & 8 & 25 & 30 & 46 & 2 & 74 & 5 & 31 \\
\hline 0 & 1 & 0 & 50 & 70 & 1 & 0 & 45 & 58 \\
\hline 0 & 1 & 0 & 51 & 68 & 1 & 0 & 56 & 50 \\
\hline 3 & 7 & 4 & 47 & 60 & 1 & 0 & 81 & 57 \\
\hline 3 & 8 & 6 & 55 & 62 & 1 & 0 & 82 & 54 \\
\hline 0 & 0 & 7 & 14 & 20 & 0 & 70 & 57 & 24 \\
\hline 0 & 1 & 7 & 16 & 44 & 1 & 75 & 61 & 27 \\
\hline 0 & 2 & 7 & 43 & 47 & 1 & 75 & 98 & 31 \\
\hline 2 & 5 & 11 & 36 & 53 & 2 & 79 & 54 & 45 \\
\hline 0 & 0 & 0 & 0 & 79 & 0 & 80 & 100 & 0 \\
\hline 0 & 0 & 0 & 0 & 79 & 0 & 80 & 100 & 0 \\
\hline 0 & 0 & 0 & 8 & 76 & 0 & 90 & 100 & 0 \\
\hline 0 & 1 & 0 & 8 & 76 & 0 & 100 & 100 & 0 \\
\hline 0 & 2 & 1 & 13 & 13 & 5 & 51 & 0 & 0 \\
\hline 2 & 8 & 11 & 22 & 12 & 3 & 54 & 11 & 100 \\
\hline 2 & 9 & 6 & 33 & 22 & 3 & 100 & 11 & 100 \\
\hline 2 & 9 & 6 & 35 & 23 & 3 & 88 & 88 & 100 \\
\hline 3 & 5 & 0 & 47 & 98 & 3 & 99 & 5 & 44 \\
\hline 3 & 6 & 1 & 38 & 75 & 5 & 99 & 11 & 16 \\
\hline 3 & 10 & 1 & 35 & 100 & 5 & 99 & 86 & 5 \\
\hline 3 & 13 & 3 & 53 & 81 & 5 & 99 & 85 & 4 \\
\hline 0 & 3 & 0 & 27 & 51 & 0 & 78 & 34 & 0 \\
\hline 0 & 16 & 11 & 31 & 38 & 1 & 70 & 34 & 0 \\
\hline 0 & 7 & 11 & 54 & 48 & 0 & 100 & 35 & 0 \\
\hline 0 & 7 & 0 & 46 & 46 & 0 & 100 & 100 & 0 \\
\hline 2 & 2 & 18 & 25 & 29 & 0 & 36 & 2 & 0 \\
\hline 2 & 2 & 22 & 22 & 33 & 0 & 40 & 0 & 0 \\
\hline 0 & 2 & 22 & 23 & 30 & 1 & 47 & 3 & 0 \\
\hline 0 & 2 & 16 & 23 & 32 & 1 & 47 & 3 & 0 \\
\hline 0 & 0 & 51 & 14 & 14 & 1 & 0 & 100 & 100 \\
\hline 0 & 0 & 51 & 16 & 17 & 1 & 100 & 100 & 100 \\
\hline 0 & 0 & 51 & 14 & 16 & 1 & 92 & 100 & 100 \\
\hline 0 & 0 & 51 & 14 & 22 & 1 & 92 & 100 & 100 \\
\hline 0 & 3 & 0 & 20 & 66 & 0 & 100 & 91 & 60 \\
\hline 0 & 6 & 0 & 50 & 67 & 0 & 100 & 1 & 0 \\
\hline
\end{tabular}




\begin{tabular}{|c|c|c|c|c|c|c|c|c|}
\hline 0 & 7 & 0 & 57 & 70 & 0 & 100 & 1 & 70 \\
\hline 0 & 6 & 0 & 52 & 71 & 0 & 100 & 6 & 0 \\
\hline 5 & 4 & 9 & 14 & 46 & 0 & 0 & 21 & 33 \\
\hline 2 & 8 & 18 & 17 & 57 & 0 & 0 & 0 & 74 \\
\hline 12 & 4 & 18 & 21 & 64 & 0 & 0 & 1 & 90 \\
\hline 15 & 5 & 37 & 21 & 68 & 0 & 0 & 100 & 90 \\
\hline 0 & 0 & 0 & 7 & 63 & 0 & 33 & 20 & 11 \\
\hline 0 & 0 & 0 & 7 & 23 & 0 & 100 & 66 & 24 \\
\hline 0 & 2 & 0 & 11 & 26 & 0 & 100 & 64 & 24 \\
\hline 0 & 3 & 0 & 13 & 24 & 0 & 100 & 82 & 36 \\
\hline 0 & 0 & 0 & 36 & 66 & 0 & 0 & 65 & 19 \\
\hline 0 & 2 & 0 & 57 & 65 & 1 & 0 & 64 & 23 \\
\hline 0 & 5 & 6 & 61 & 64 & 5 & 0 & 63 & 26 \\
\hline 0 & 4 & 0 & 60 & 65 & 1 & 0 & 63 & 25 \\
\hline 0 & 3 & 40 & 5 & 50 & 0 & 0 & 0 & 67 \\
\hline 0 & 2 & 18 & 8 & 59 & 2 & 0 & 0 & 0 \\
\hline 0 & 1 & 18 & 34 & 62 & 2 & 0 & 0 & 0 \\
\hline 0 & 0 & 0 & 34 & 66 & 2 & 0 & 35 & 0 \\
\hline 0 & 1 & 0 & 23 & 72 & 1 & 0 & 27 & 0 \\
\hline 0 & 3 & 0 & 45 & 74 & 0 & 0 & 28 & 0 \\
\hline 0 & 3 & 0 & 52 & 73 & 0 & 0 & 100 & 0 \\
\hline 0 & 3 & 0 & 54 & 80 & 0 & 0 & 100 & 0 \\
\hline 0 & 0 & 0 & 39 & 40 & 0 & 65 & 0 & 100 \\
\hline 0 & 0 & 0 & 26 & 7 & 0 & 99 & 0 & 100 \\
\hline 0 & 0 & 0 & 36 & 24 & 0 & 100 & 0 & 100 \\
\hline 0 & 0 & 0 & 50 & 20 & 0 & 81 & 98 & 100 \\
\hline 0 & 1 & 0 & 65 & 68 & 0 & 0 & 57 & 11 \\
\hline 0 & 3 & 0 & 79 & 85 & 0 & 0 & 0 & 14 \\
\hline 0 & 4 & 0 & 61 & 85 & 0 & 0 & 43 & 19 \\
\hline 0 & 6 & 0 & 61 & 84 & 0 & 0 & 42 & 100 \\
\hline 0 & 0 & 0 & 23 & 64 & 0 & 76 & 43 & 56 \\
\hline 0 & 0 & 0 & 65 & 56 & 0 & 100 & 63 & 56 \\
\hline 0 & 0 & 3 & 57 & 49 & 0 & 100 & 62 & 81 \\
\hline 0 & 0 & 3 & 57 & 49 & 0 & 100 & 70 & 96 \\
\hline 0 & 0 & 0 & 0 & 68 & 0 & 0 & 0 & 0 \\
\hline 0 & 0 & 0 & 29 & 34 & 0 & 0 & 0 & 0 \\
\hline 1 & 1 & 0 & 32 & 40 & 5 & 0 & 0 & 0 \\
\hline 1 & 1 & 0 & 29 & 46 & 6 & 0 & 12 & 0 \\
\hline 0 & 2 & 6 & 7 & 31 & 2 & 100 & 99 & 0 \\
\hline 0 & 4 & 7 & 30 & 42 & 5 & 100 & 99 & 0 \\
\hline 0 & 15 & 8 & 37 & 47 & 5 & 100 & 95 & 0 \\
\hline 0 & 19 & 8 & 34 & 45 & 5 & 100 & 95 & 0 \\
\hline
\end{tabular}




\begin{tabular}{|c|c|c|c|c|c|c|c|c|}
\hline 0 & 0 & 0 & 0 & 20 & 1 & 0 & 1 & 9 \\
\hline 0 & 0 & 0 & 0 & 20 & 3 & 24 & 3 & 2 \\
\hline 0 & 1 & 0 & 0 & 100 & 3 & 54 & 3 & 1 \\
\hline 0 & 1 & 0 & 0 & 100 & 3 & 34 & 3 & 1 \\
\hline 0 & 0 & 0 & 2 & 22 & 2 & 0 & 0 & 20 \\
\hline 0 & 0 & 9 & 13 & 29 & 1 & 0 & 53 & 50 \\
\hline 0 & 0 & 9 & 34 & 41 & 1 & 0 & 77 & 52 \\
\hline 0 & 0 & 24 & 16 & 30 & 4 & 0 & 77 & 65 \\
\hline 4 & 2 & 84 & 14 & 45 & 0 & 0 & 87 & 77 \\
\hline 4 & 4 & 84 & 34 & 48 & 0 & 0 & 86 & 79 \\
\hline 4 & 4 & 84 & 41 & 56 & 0 & 0 & 85 & 79 \\
\hline 4 & 5 & 100 & 41 & 56 & 0 & 0 & 100 & 100 \\
\hline 0 & 1 & 8 & 10 & 43 & 3 & 0 & 90 & 0 \\
\hline 8 & 10 & 53 & 12 & 41 & 16 & 0 & 100 & 100 \\
\hline 8 & 11 & 53 & 9 & 40 & 16 & 0 & 100 & 100 \\
\hline 8 & 11 & 53 & 9 & 44 & 16 & 0 & 100 & 100 \\
\hline 0 & 0 & 7 & 30 & 37 & 1 & 0 & 100 & 0 \\
\hline 0 & 0 & 22 & 26 & 39 & 2 & 0 & 100 & 0 \\
\hline 0 & 1 & 22 & 51 & 39 & 2 & 0 & 100 & 0 \\
\hline 0 & 4 & 22 & 56 & 42 & 2 & 0 & 100 & 0 \\
\hline 0 & 0 & 0 & 9 & 13 & 0 & 0 & 83 & 0 \\
\hline 1 & 5 & 0 & 12 & 14 & 2 & 0 & 83 & 0 \\
\hline 4 & 8 & 0 & 17 & 15 & 7 & 0 & 29 & 0 \\
\hline 4 & 8 & 0 & 20 & 15 & 8 & 0 & 50 & 0 \\
\hline 0 & 2 & 0 & 46 & 67 & 0 & 95 & 2 & 0 \\
\hline 0 & 2 & 0 & 43 & 63 & 0 & 76 & 2 & 0 \\
\hline 0 & 2 & 1 & 37 & 65 & 1 & 80 & 0 & 0 \\
\hline 0 & 2 & 1 & 38 & 75 & 1 & 81 & 0 & 0 \\
\hline 0 & 0 & 6 & 33 & 98 & 0 & 0 & 0 & 0 \\
\hline 0 & 2 & 6 & 7 & 94 & 0 & 0 & 100 & 0 \\
\hline 0 & 1 & 6 & 6 & 97 & 0 & 0 & 100 & 100 \\
\hline 0 & 1 & 6 & 6 & 94 & 0 & 0 & 100 & 100 \\
\hline 0 & 1 & 0 & 13 & 12 & 3 & 0 & 0 & 19 \\
\hline 0 & 3 & 1 & 16 & 19 & 4 & 0 & 8 & 19 \\
\hline 0 & 7 & 1 & 31 & 20 & 5 & 0 & 0 & 0 \\
\hline 0 & 4 & 31 & 37 & 25 & 4 & 0 & 0 & 0 \\
\hline 0 & 0 & 0 & 9 & 59 & 0 & 0 & 100 & 100 \\
\hline 0 & 1 & 0 & 14 & 69 & 15 & 0 & 100 & 100 \\
\hline 0 & 17 & 0 & 17 & 70 & 18 & 0 & 100 & 100 \\
\hline 0 & 20 & 2 & 18 & 70 & 18 & 0 & 81 & 100 \\
\hline 67 & 15 & 14 & 40 & 0 & 0 & 0 & 100 & 4 \\
\hline 67 & 15 & 18 & 1 & 72 & 4 & 0 & 100 & 100 \\
\hline
\end{tabular}




\begin{tabular}{|c|c|c|c|c|c|c|c|c|}
\hline 67 & 20 & 18 & 99 & 71 & 4 & 0 & 100 & 100 \\
\hline 67 & 20 & 18 & 99 & 70 & 4 & 0 & 100 & 100 \\
\hline 0 & 0 & 20 & 0 & 11 & 0 & 89 & 100 & 100 \\
\hline 0 & 0 & 22 & 0 & 12 & 0 & 100 & 100 & 100 \\
\hline 0 & 4 & 22 & 0 & 11 & 0 & 100 & 99 & 100 \\
\hline 0 & 0 & 0 & 0 & 59 & 0 & 100 & 99 & 100 \\
\hline 0 & 0 & 2 & 5 & 21 & 0 & 100 & 0 & 0 \\
\hline 0 & 0 & 2 & 14 & 20 & 0 & 100 & 84 & 100 \\
\hline 0 & 2 & 2 & 17 & 23 & 1 & 100 & 98 & 43 \\
\hline 0 & 2 & 2 & 17 & 23 & 1 & 100 & 98 & 43 \\
\hline 0 & 0 & 0 & 7 & 27 & 0 & 0 & 0 & 0 \\
\hline 0 & 0 & 0 & 20 & 42 & 0 & 0 & 0 & 0 \\
\hline 0 & 0 & 2 & 19 & 52 & 0 & 0 & 0 & 0 \\
\hline 0 & 5 & 0 & 19 & 52 & 0 & 0 & 0 & 0 \\
\hline 0 & 0 & 0 & 6 & 64 & 0 & 0 & 0 & 100 \\
\hline 0 & 0 & 0 & 5 & 64 & 0 & 0 & 0 & 100 \\
\hline 0 & 0 & 0 & 2 & 63 & 0 & 0 & 0 & 100 \\
\hline 0 & 0 & 100 & 0 & 62 & 0 & 0 & 0 & 100 \\
\hline 0 & 2 & 0 & 2 & 22 & 3 & 0 & 0 & 100 \\
\hline 0 & 7 & 0 & 9 & 80 & 8 & 0 & 0 & 100 \\
\hline 0 & 1 & 0 & 28 & 64 & 8 & 0 & 0 & 0 \\
\hline 0 & 1 & 5 & 27 & 69 & 9 & 0 & 62 & 0 \\
\hline 0 & 0 & 0 & 1 & 62 & 0 & 0 & 0 & 0 \\
\hline 0 & 0 & 100 & 2 & 66 & 0 & 0 & 0 & 0 \\
\hline 0 & 0 & 100 & 3 & 73 & 0 & 0 & 0 & 0 \\
\hline 0 & 0 & 100 & 4 & 72 & 0 & 0 & 0 & 0 \\
\hline 0 & 0 & 0 & 11 & 45 & 0 & 90 & 0 & 0 \\
\hline 0 & 0 & 0 & 80 & 56 & 0 & 100 & 0 & 0 \\
\hline 0 & 0 & 0 & 79 & 56 & 4 & 100 & 0 & 0 \\
\hline 0 & 0 & 0 & 79 & 62 & 4 & 100 & 0 & 27 \\
\hline 0 & 0 & 2 & 5 & 85 & 5 & 0 & 100 & 100 \\
\hline 7 & 9 & 6 & 5 & 84 & 5 & 0 & 0 & 100 \\
\hline 7 & 9 & 6 & 4 & 85 & 5 & 0 & 0 & 100 \\
\hline 7 & 9 & 6 & 4 & 85 & 5 & 0 & 0 & 100 \\
\hline 0 & 2 & 0 & 19 & 55 & 6 & 0 & 0 & 47 \\
\hline 0 & 2 & 1 & 38 & 42 & 6 & 0 & 0 & 42 \\
\hline 0 & 2 & 0 & 44 & 45 & 6 & 0 & 0 & 44 \\
\hline 0 & 2 & 0 & 42 & 45 & 6 & 0 & 0 & 44 \\
\hline 0 & 0 & 0 & 44 & 43 & 0 & 60 & 0 & 100 \\
\hline 0 & 0 & 0 & 9 & 45 & 0 & 88 & 14 & 100 \\
\hline 0 & 0 & 0 & 9 & 50 & 0 & 80 & 18 & 100 \\
\hline 0 & 0 & 0 & 10 & 52 & 0 & 81 & 17 & 100 \\
\hline
\end{tabular}




\begin{tabular}{|c|c|c|c|c|c|c|c|c|}
\hline 0 & 1 & 16 & 11 & 42 & 4 & 0 & 0 & 97 \\
\hline 0 & 0 & 0 & 0 & 89 & 0 & 0 & 0 & 97 \\
\hline 5 & 7 & 0 & 0 & 88 & 6 & 0 & 98 & 98 \\
\hline 0 & 17 & 0 & 0 & 88 & 6 & 0 & 98 & 91 \\
\hline 0 & 2 & 0 & 77 & 82 & 0 & 0 & 86 & 100 \\
\hline 0 & 4 & 0 & 77 & 95 & 0 & 0 & 100 & 100 \\
\hline 0 & 4 & 0 & 82 & 96 & 0 & 0 & 100 & 100 \\
\hline 0 & 4 & 0 & 81 & 95 & 0 & 0 & 100 & 99 \\
\hline 0 & 0 & 0 & 0 & 51 & 0 & 0 & 0 & 0 \\
\hline 0 & 0 & 0 & 0 & 52 & 0 & 0 & 0 & 100 \\
\hline 0 & 0 & 0 & 0 & 51 & 0 & 0 & 0 & 0 \\
\hline 0 & 0 & 0 & 0 & 51 & 0 & 0 & 0 & 0 \\
\hline 0 & 0 & 0 & 57 & 37 & 0 & 0 & 0 & 0 \\
\hline 0 & 4 & 0 & 66 & 51 & 0 & 0 & 100 & 100 \\
\hline 0 & 16 & 0 & 76 & 70 & 0 & 0 & 100 & 100 \\
\hline 0 & 15 & 0 & 92 & 87 & 0 & 0 & 100 & 100 \\
\hline 0 & 0 & 0 & 11 & 40 & 0 & 0 & 0 & 92 \\
\hline 0 & 65 & 0 & 11 & 41 & 0 & 0 & 27 & 92 \\
\hline 0 & 65 & 0 & 16 & 46 & 0 & 0 & 100 & 100 \\
\hline 0 & 65 & 0 & 16 & 46 & 0 & 0 & 100 & 100 \\
\hline 0 & 0 & 0 & 8 & 16 & 1 & 0 & 80 & 79 \\
\hline 0 & 5 & 32 & 27 & 78 & 5 & 0 & 100 & 100 \\
\hline 0 & 3 & 2 & 24 & 6 & 5 & 0 & 100 & 100 \\
\hline 0 & 3 & 2 & 100 & 18 & 2 & 0 & 100 & 100 \\
\hline 0 & 0 & 86 & 16 & 18 & 0 & 0 & 0 & 0 \\
\hline 0 & 0 & 86 & 18 & 18 & 0 & 0 & 0 & 0 \\
\hline 0 & 0 & 0 & 18 & 18 & 0 & 0 & 0 & 100 \\
\hline 0 & 0 & 0 & 18 & 18 & 0 & 0 & 0 & 100 \\
\hline 0 & 2 & 0 & 14 & 12 & 0 & 0 & 0 & 0 \\
\hline 0 & 2 & 0 & 14 & 12 & 0 & 0 & 0 & 0 \\
\hline 0 & 2 & 0 & 14 & 14 & 0 & 0 & 0 & 0 \\
\hline 0 & 2 & 0 & 14 & 14 & 0 & 0 & 0 & 0 \\
\hline 0 & 0 & 0 & 11 & 23 & 0 & 0 & 0 & 0 \\
\hline 0 & 0 & 0 & 13 & 17 & 0 & 0 & 0 & 0 \\
\hline 0 & 0 & 0 & 13 & 21 & 0 & 0 & 0 & 0 \\
\hline 0 & 0 & 0 & 16 & 21 & 0 & 0 & 0 & 0 \\
\hline 0 & 0 & 0 & 0 & 21 & 0 & 0 & 0 & 0 \\
\hline 0 & 0 & 0 & 0 & 20 & 0 & 0 & 0 & 0 \\
\hline 0 & 0 & 0 & 0 & 19 & 0 & 0 & 0 & 0 \\
\hline 0 & 0 & 0 & 0 & 20 & 0 & 0 & 9 & 0 \\
\hline 0 & 20 & 0 & 1 & 79 & 0 & 0 & 0 & 100 \\
\hline 0 & 25 & 0 & 7 & 76 & 0 & 0 & 0 & 100 \\
\hline
\end{tabular}




\begin{tabular}{|c|c|c|c|c|c|c|c|c|}
\hline 0 & 0 & 0 & 8 & 76 & 0 & 0 & 0 & 100 \\
\hline 0 & 0 & 0 & 9 & 75 & 0 & 0 & 8 & 100 \\
\hline 0 & 0 & 17 & 2 & 74 & 0 & 0 & 0 & 0 \\
\hline 0 & 0 & 13 & 3 & 77 & 0 & 0 & 95 & 100 \\
\hline 0 & 0 & 17 & 3 & 81 & 0 & 0 & 94 & 100 \\
\hline 0 & 0 & 4 & 3 & 80 & 0 & 0 & 95 & 100 \\
\hline 0 & 0 & 0 & 1 & 4 & 0 & 100 & 0 & 100 \\
\hline 0 & 0 & 0 & 1 & 7 & 2 & 100 & 0 & 81 \\
\hline 0 & 2 & 4 & 9 & 11 & 3 & 100 & 100 & 100 \\
\hline 0 & 0 & 0 & 14 & 26 & 0 & 100 & 100 & 100 \\
\hline 0 & 0 & 0 & 0 & 66 & 0 & 0 & 0 & 0 \\
\hline 0 & 0 & 0 & 0 & 63 & 0 & 0 & 0 & 0 \\
\hline 0 & 0 & 0 & 12 & 66 & 0 & 0 & 100 & 100 \\
\hline 0 & 0 & 0 & 15 & 67 & 0 & 0 & 100 & 100 \\
\hline 0 & 0 & 0 & 3 & 44 & 0 & 0 & 0 & 0 \\
\hline 0 & 0 & 0 & 6 & 47 & 0 & 0 & 0 & 0 \\
\hline 0 & 0 & 0 & 8 & 57 & 0 & 0 & 13 & 0 \\
\hline 0 & 0 & 0 & 8 & 57 & 0 & 0 & 12 & 0 \\
\hline 0 & 1 & 0 & 3 & 12 & 6 & 0 & 0 & 0 \\
\hline 0 & 0 & 10 & 32 & 11 & 6 & 0 & 0 & 0 \\
\hline 1 & 1 & 10 & 36 & 13 & 0 & 0 & 0 & 0 \\
\hline 6 & 6 & 15 & 30 & 30 & 0 & 0 & 0 & 0 \\
\hline 0 & 0 & 0 & 14 & 46 & 1 & 0 & 0 & 0 \\
\hline 0 & 0 & 0 & 4 & 44 & 2 & 0 & 0 & 0 \\
\hline 0 & 1 & 1 & 4 & 44 & 2 & 0 & 100 & 100 \\
\hline 0 & 1 & 0 & 15 & 42 & 2 & 0 & 100 & 100 \\
\hline 0 & 0 & 0 & 7 & 27 & 0 & 0 & 100 & 100 \\
\hline 0 & 0 & 0 & 4 & 15 & 0 & 0 & 100 & 100 \\
\hline 0 & 0 & 0 & 16 & 17 & 3 & 0 & 100 & 71 \\
\hline 0 & 0 & 0 & 12 & 14 & 3 & 0 & 100 & 80 \\
\hline
\end{tabular}




$\begin{array}{llll}\text { X21 } & \text { X22 } & \text { X23 } & \text { X24 } \\ 3 & 27 & 1 & 82 \\ 3 & 39 & 2 & 10 \\ 3 & 79 & 5 & 2 \\ 3 & 76 & 6 & 9 \\ 0 & 87 & 1 & 100 \\ 8 & 88 & 1 & 19 \\ 72 & 95 & 2 & 8 \\ 78 & 95 & 3 & 11 \\ 0 & 63 & 5 & 6 \\ 1 & 65 & 6 & 1 \\ 98 & 62 & 7 & 1 \\ 32 & 80 & 18 & 5 \\ 96 & 55 & 3 & 18 \\ 100 & 53 & 3 & 25 \\ 29 & 71 & 7 & 70 \\ 22 & 70 & 6 & 51 \\ 100 & 91 & 0 & 11 \\ 93 & 96 & 0 & 3 \\ 93 & 98 & 3 & 1 \\ 93 & 98 & 7 & 1 \\ 0 & 44 & 0 & 0 \\ 8 & 93 & 40 & 0 \\ 8 & 65 & 31 & 3 \\ 8 & 85 & 35 & 9 \\ 29 & 63 & 1 & 26 \\ 29 & 71 & 9 & 1 \\ 100 & 71 & 13 & 1 \\ 100 & 73 & 11 & 1 \\ 0 & 37 & 0 & 15 \\ 0 & 66 & 0 & 14 \\ 0 & 84 & 2 & 14 \\ 0 & 85 & 2 & 14 \\ 97 & 72 & 2 & 3 \\ 100 & 75 & 5 & 6 \\ 100 & 81 & 42 & 5 \\ 100 & 81 & 49 & 5 \\ 0 & 91 & 2 & 100 \\ 0 & 95 & 35 & 7 \\ 03 & & & \\ & 53\end{array}$




\begin{tabular}{|c|c|c|}
\hline 0 & 97 & 38 \\
\hline 0 & 98 & 40 \\
\hline 0 & 84 & 11 \\
\hline 0 & 80 & 14 \\
\hline 1 & 84 & 19 \\
\hline 1 & 88 & 21 \\
\hline 8 & 70 & 3 \\
\hline 58 & 74 & 3 \\
\hline 24 & 66 & 0 \\
\hline 35 & 42 & 0 \\
\hline 0 & 88 & 10 \\
\hline 0 & 86 & 2 \\
\hline 4 & 90 & 7 \\
\hline 4 & 91 & 11 \\
\hline 32 & 47 & 0 \\
\hline 37 & 100 & 46 \\
\hline 0 & 93 & 34 \\
\hline 74 & 92 & 40 \\
\hline 100 & 67 & 0 \\
\hline 6 & 60 & 1 \\
\hline 100 & 63 & 2 \\
\hline 100 & 66 & 2 \\
\hline 30 & 75 & 0 \\
\hline 0 & 87 & 0 \\
\hline 0 & 95 & 49 \\
\hline 0 & 96 & 58 \\
\hline 0 & 81 & 0 \\
\hline 0 & 88 & 11 \\
\hline 43 & 84 & 19 \\
\hline 47 & 83 & 44 \\
\hline 0 & 52 & 0 \\
\hline 0 & 91 & 5 \\
\hline 0 & 89 & 35 \\
\hline 0 & 99 & 15 \\
\hline 0 & 27 & 0 \\
\hline 0 & 53 & 8 \\
\hline 0 & 36 & 5 \\
\hline 12 & 57 & 9 \\
\hline 0 & 84 & 0 \\
\hline 0 & 93 & 8 \\
\hline 0 & 99 & 13 \\
\hline 0 & 100 & 7 \\
\hline
\end{tabular}




$\begin{array}{llll}0 & 98 & 0 & 100 \\ 3 & 100 & 0 & 100 \\ 3 & 96 & 0 & 100 \\ 3 & 96 & 0 & 100 \\ 36 & 57 & 0 & 2 \\ 53 & 58 & 0 & 1 \\ 77 & 59 & 17 & 1 \\ 77 & 63 & 17 & 1 \\ 0 & 90 & 6 & 18 \\ 0 & 93 & 7 & 13 \\ 0 & 95 & 6 & 11 \\ 0 & 97 & 20 & 11 \\ 82 & 0 & 21 & 0 \\ 0 & 90 & 8 & 0 \\ 0 & 93 & 24 & 0 \\ 0 & 90 & 21 & 0 \\ 100 & 93 & 3 & 0 \\ 100 & 67 & 2 & 0 \\ 100 & 57 & 6 & 2 \\ 100 & 53 & 7 & 2 \\ 0 & 78 & 0 & 0 \\ 0 & 64 & 0 & 0 \\ 29 & 55 & 20 & 0 \\ 50 & 77 & 50 & 0 \\ 0 & 26 & 0 & 11 \\ 0 & 72 & 1 & 10 \\ 0 & 81 & 21 & 10 \\ 0 & 59 & 50 & 10 \\ 0 & 84 & 0 & 0 \\ 0 & 88 & 0 & 0 \\ 100 & 84 & 0 & 0 \\ 100 & 83 & 6 & 0 \\ 0 & 63 & 0 & 0 \\ 0 & 69 & 1 & 0 \\ 0 & 99 & 4 & 0 \\ 0 & 93 & 0 & 0 \\ 0 & 54 & 0 & 0 \\ 0 & 60 & 30 & 0 \\ 0 & 59 & 36 & 0 \\ 0 & 70 & 41 & 0 \\ 1 & 100 & 0 & 100 \\ 100 & 100 & 0 & 100\end{array}$




\begin{tabular}{|c|c|c|}
\hline 100 & 94 & 38 \\
\hline 100 & 94 & 67 \\
\hline 100 & 46 & 0 \\
\hline 100 & 77 & 0 \\
\hline 99 & 80 & 0 \\
\hline 99 & 82 & 0 \\
\hline 0 & 47 & 0 \\
\hline 0 & 66 & 0 \\
\hline 14 & 73 & 34 \\
\hline 14 & 72 & 37 \\
\hline 0 & 75 & 0 \\
\hline 0 & 93 & 0 \\
\hline 0 & 95 & 15 \\
\hline 0 & 90 & 20 \\
\hline 0 & 95 & 0 \\
\hline 0 & 99 & 0 \\
\hline 0 & 70 & 0 \\
\hline 0 & 100 & 0 \\
\hline 0 & 65 & 7 \\
\hline 0 & 99 & 1 \\
\hline 0 & 95 & 5 \\
\hline 0 & 93 & 23 \\
\hline 98 & 90 & 2 \\
\hline 98 & 93 & 3 \\
\hline 0 & 92 & 2 \\
\hline 0 & 94 & 2 \\
\hline 0 & 62 & 0 \\
\hline 0 & 77 & 0 \\
\hline 0 & 100 & 23 \\
\hline 0 & 87 & 35 \\
\hline 0 & 5 & 0 \\
\hline 0 & 20 & 3 \\
\hline 0 & 92 & 0 \\
\hline 0 & 92 & 2 \\
\hline 0 & 48 & 0 \\
\hline 0 & 68 & 0 \\
\hline 0 & 76 & 5 \\
\hline 0 & 86 & 6 \\
\hline 0 & 73 & 1 \\
\hline 0 & 78 & 8 \\
\hline 0 & 82 & 21 \\
\hline 0 & 83 & 15 \\
\hline
\end{tabular}




$\begin{array}{llll}0 & 88 & 7 & 100 \\ 0 & 87 & 7 & 100 \\ 98 & 97 & 7 & 100 \\ 98 & 94 & 7 & 0 \\ 87 & 92 & 0 & 48 \\ 100 & 93 & 0 & 13 \\ 100 & 83 & 22 & 13 \\ 100 & 89 & 22 & 13 \\ 0 & 100 & 0 & 0 \\ 0 & 100 & 0 & 2 \\ 0 & 84 & 0 & 0 \\ 0 & 83 & 0 & 0 \\ 0 & 87 & 0 & 0 \\ 100 & 78 & 0 & 0 \\ 100 & 100 & 0 & 85 \\ 100 & 100 & 0 & 22 \\ 0 & 100 & 0 & 100 \\ 0 & 100 & 0 & 100 \\ 100 & 100 & 0 & 100 \\ 100 & 88 & 0 & 100 \\ 80 & 100 & 0 & 0 \\ 100 & 72 & 41 & 0 \\ 100 & 100 & 40 & 0 \\ 100 & 100 & 42 & 0 \\ 0 & 74 & 0 & 0 \\ 0 & 82 & 6 & 100 \\ 0 & 73 & 5 & 100 \\ 0 & 71 & 8 & 100 \\ 0 & 91 & 0 & 0 \\ 0 & 94 & 34 & 0 \\ 0 & 89 & 32 & 0 \\ 0 & 88 & 32 & 0 \\ 0 & 67 & 0 & 0 \\ 0 & 88 & 53 & 0 \\ 0 & 88 & 45 & 0 \\ 0 & 45 & 40 & 0 \\ 0 & 89 & 0 & 2 \\ 0 & 82 & 0 & 0 \\ 0 & 93 & 0 & 0 \\ 0 & 72 & 0 & 0 \\ 0 & 82 & 0 & 0 \\ 0 & 53 & 4 & 0\end{array}$




\begin{tabular}{|c|c|c|}
\hline 0 & 87 & 5 \\
\hline 0 & 90 & 5 \\
\hline 0 & 30 & 8 \\
\hline 95 & 91 & 6 \\
\hline 94 & 87 & 16 \\
\hline 95 & 93 & 16 \\
\hline 98 & 57 & 3 \\
\hline 100 & 73 & 3 \\
\hline 100 & 83 & 5 \\
\hline 100 & 86 & 3 \\
\hline 0 & 58 & 0 \\
\hline 0 & 84 & 0 \\
\hline 0 & 83 & 0 \\
\hline 0 & 82 & 0 \\
\hline 0 & 80 & 1 \\
\hline 0 & 69 & 1 \\
\hline 0 & 74 & 8 \\
\hline 0 & 72 & 7 \\
\hline 0 & 70 & 2 \\
\hline 0 & 72 & 6 \\
\hline 0 & 100 & 7 \\
\hline 0 & 99 & 9 \\
\hline 0 & 100 & 0 \\
\hline 0 & 99 & 8 \\
\hline 0 & 100 & 2 \\
\hline 0 & 100 & 1 \\
\hline 0 & 17 & 2 \\
\hline 0 & 51 & 1 \\
\hline 0 & 80 & 6 \\
\hline 0 & 96 & 13 \\
\hline
\end{tabular}


Where

Target variable $=\mathrm{Y}=$ Annual Average Concentration of $\mathrm{CO}$ in ambient air - Annual Average Concentration of Ozone in ambient air $=$ Annual Average Concentration of NOX in ambient air

Input Variables:

$\mathrm{X} 1=$ Population in thousands

$\mathrm{X} 2=$ Road Length in miles

$\mathrm{X} 3=\mathrm{DVMT}$ in thousands

$\mathrm{X} 4=$ Land Area in sq. miles

X5 = Freeway DVMT in thousands

X6 $=$ Freeway Miles

X7 = Annual average daily traffic on freeways

$\mathrm{X} 8=\%$ of Freeway Miles covered by Highway Advisory Radio (HAR)

$\mathrm{X} 9=\%$ of Freeway miles under incident detection algorithms

$\mathrm{X} 10=\%$ of Freeway miles covered by surveillance cameras (CCTV)

$\mathrm{X} 11=\%$ of Freeway miles covered by service patrols

$\mathrm{X} 12=\%$ of Arterial miles under incident detection algorithms

$\mathrm{X} 13=\%$ of Arterial miles covered by surveillance cameras (CCTV)

$\mathrm{X} 14=\%$ of Arterial miles covered by service patrols

$\mathrm{X} 15=\%$ of Signalized intersections covered by electronic survcillance 
$\mathrm{X} 16=\%$ of Signalized intersections under centralized or closed loop control

$\mathrm{X} 17=\%$ of Arterial miles covered by Highway Advisory Radio (HAR)

X18 $=\%$ of Toll collection lanes with Electronic Toll Collection capabilities

$\mathrm{X} 19=\%$ of Fixed route buses equipped with

Automatic Vehicle Location (AVL)

$\mathrm{X} 20=\%$ of Demand responsive vehicles that operate under Computer Aided Dispatch (CAD)

$\mathrm{X} 21=\%$ of Fixed route buses with electronic real-time monitoring of system components

$\mathrm{X} 22=\%$ of Vehicles under Computer Aided Dispatch (CAD) for EM

$\mathrm{X} 23=\%$ of Vehicles equipped with on-board navigation capabilities for EM (EmergencyManagement)

$\mathrm{X} 24=\%$ of Highway rail intersections under electronic surveillance 


\section{CURRICULUM VITAE}

NAME:

PERMANENT ADDRESS: C-604, Umang, Vasant Utsav

Thakur Village, Kandivli (E)

Mumbai, India-400101

CURRENT ADDRESS:

DOB:

EDUCATION \& TRAINING:

$4630 \mathrm{~S} 3^{\text {rd }}$ St., Apt.\# 2

Louisville, KY-40214

B.S., Civil Engineering
September 15, 1965, Kotputli, Jaipur, India.

Malaviya National Institute of Technology

Jaipur, India-302017

July 1981-June 1986

M.S., Civil Engineering (with Environmental Engineering as spccialization)

Indian Institute of Technology, Kharagpur, India

July 1987-January 1989

Ph.D., Civil \& Environmental Engineering University of Louisville, KY-40292

August 2005-December 2008

PROFESSIONAL SOCIETIES: UGC Fellowship, Government of India

I.I.T., Kharagpur, India

July 1987-January 1989

Grosscurth Fellowship

University of Louisville, KY

September 2005-August 2007

Research Assistant,

Dept. of Civil \& Environmental Engineering

University of Louisville, KY -40292

Scptember 2007-December 2008

Registered Professional Engineer (P.E.) with KY 